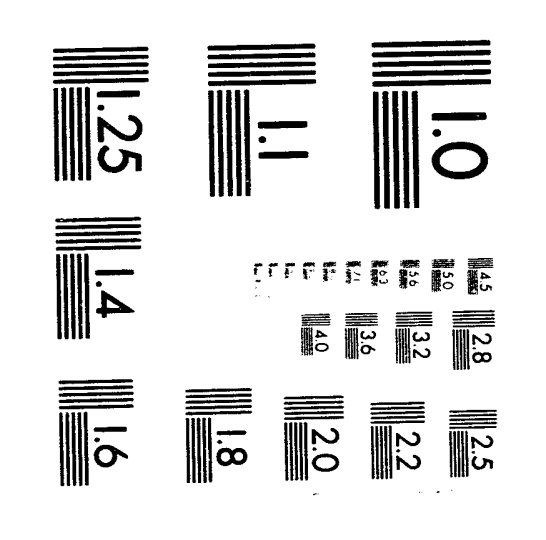

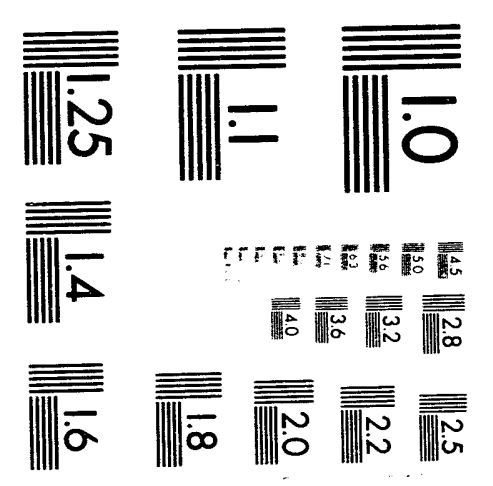



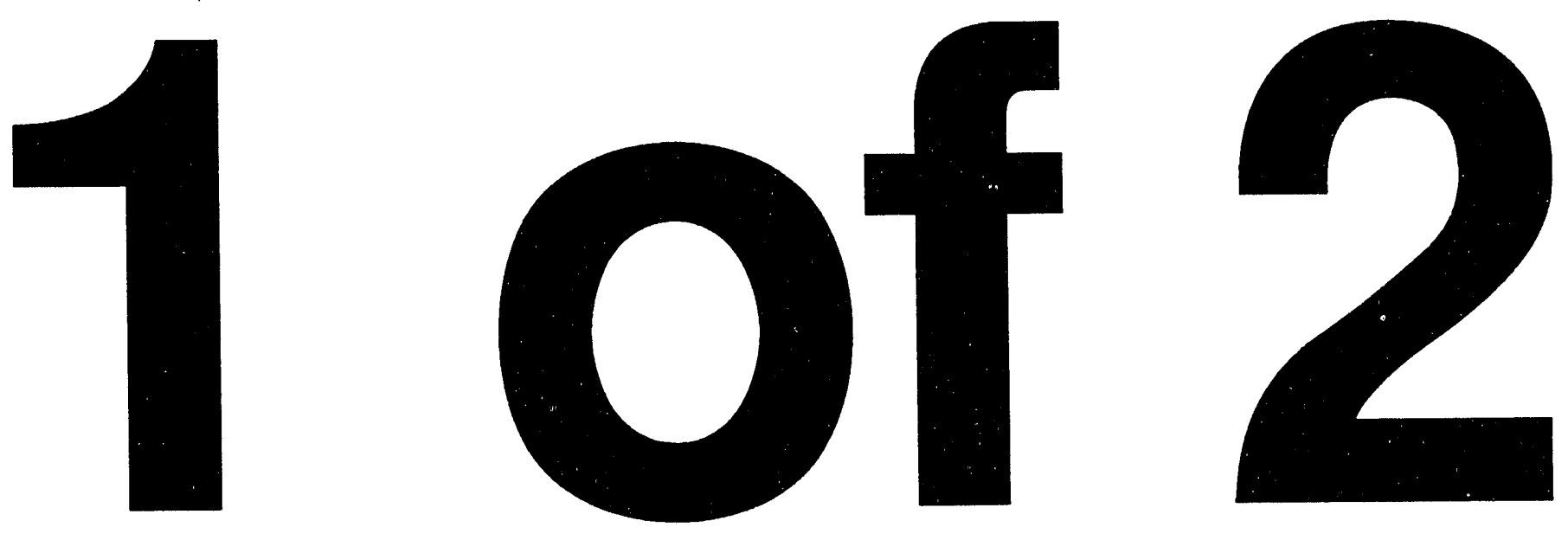
UCRL-LR-115496

Distribution Category UC-900

\title{
Optical Field Ionization of Atoms and Ions Using Ultrashort Laser Pulses
}

\author{
David Neal Fittinghoff \\ (Ph.D. Thesis)
}

Manuscript date: December 1993

LAWRENCE LIVERMORE NATIONAL LABORATORY University of California - Livermore, California • 94551 
Optical Field Ionization of Atoms and Ions Using

Ultrashort Laser Pulses

By

David Neal Fittinghoff

B.S. (University of California, Davis) 1985

M.S. (University of California, Davis) 1989

DISSERTATION

Submitted in partial satisfaction of the requirements for the degree of

DOCTOR OF PHILOSOPHY

in

Engineering-Applied Science

in the

GRADUATE DIVISION

of the

UNIVERSITY OF CALIFORNIA

DAVIS

Approved:

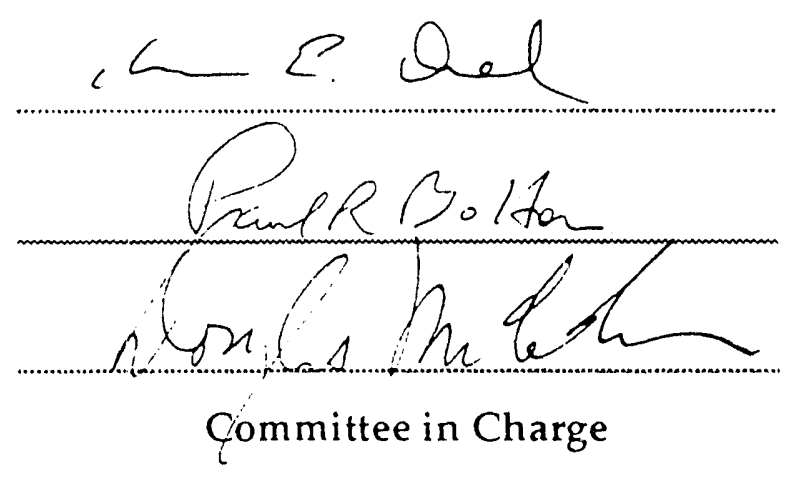

1993 


\section{Dedicated to my dear wife}

Lisa

to my family

Nicholas, Sandra, Catherine and Stephen

and to

Frank and Lyla 


\section{ACKNOWLEDGMENTS}

Many people helped and influenced my work on this Ph.D. dissertation I thank them all.

I thank my wife Lisa. Her love and patience guided me on this arcane journey when all reason failed.

I also thank my parents Nicholas and Sandra Fittinghoff, my brother Stephen and my sister Catherine for their support and encouragement and for knowing the value of education.

I will always be grateful to my grandfather Frank Riley for wisdom beyond his words, and to my grandmother Lyla Riley for her boundless love.

My research advisor, Doctor Paul Bolton, deserves more praise than I can express for his great ideas and his love of science.

My academic advisor, Professor Ann Orel, gave me uniformly good advice and I thank her for watching out for an experimentalist. I also thank Douglas McColm for his kindness and help.

The theories discussed in this dissertation would have been impenetrable without the help and contributions of Britton Chang and Kenneth Kulander, and I thank them for their contributions. I also thank Paul Corkum for the use of his calculations.

Finally, I thank David Gold, Linn Van Woerkom, Bill White, Jim Hunter, Jim Bonlie and Dwight Price for their help with the lasers. 


\section{TABLE OF CONTENTS}

TITLE PAGE

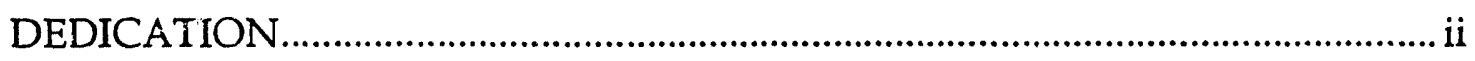

ACKNOWLEDGMENTS .................................................................................ii

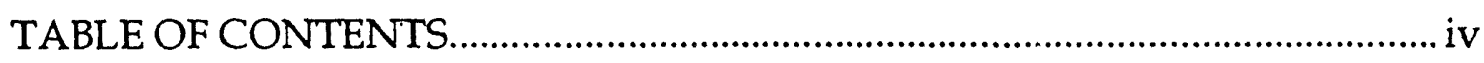

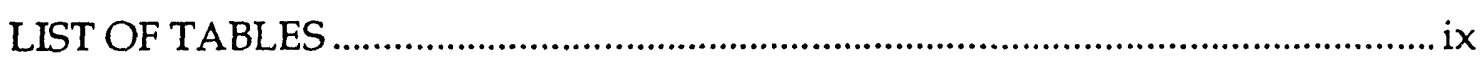

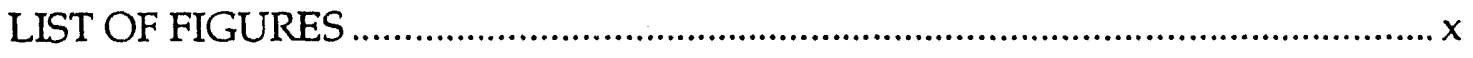

ABSTRACT

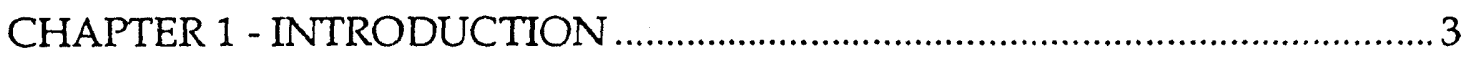

1.1 Preface

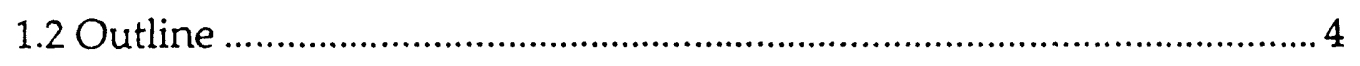

CHAPTER 2 - AN INTRODUCTION TO OPTICAL FIELD IONIZATION ....... 6

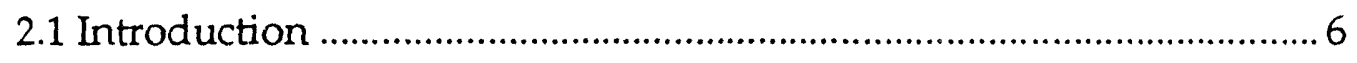

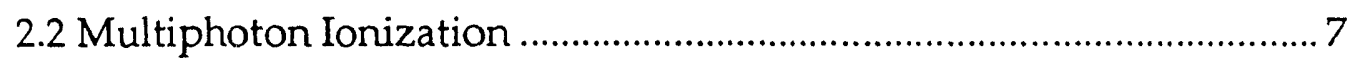

2.3 Tunneling Ionization...................................................................

2.4 The Single Active Electron Model........................................................ 14

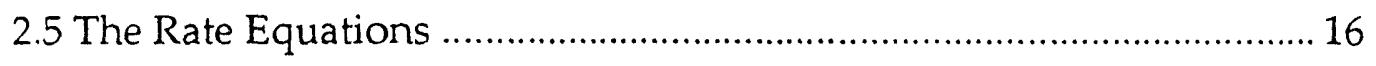


2.6 The Focal Volume.

18

2.7 Polarization Dependence of Irradiance and Units

20

Chapter 3-ANALYTIC YIELD SOLUTIONS FOR SEQUENTIAL

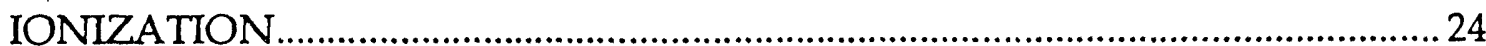

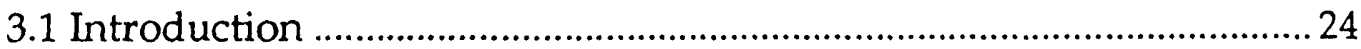

3.2 Analytic Solutions to the Rate Equations................................................2

3.3 Threshold and Saturation Irradiances.......................................................33

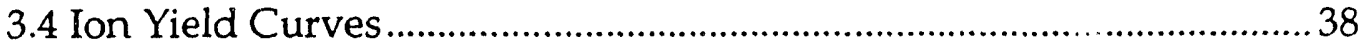

3.5 Low Irradiance Limit of the Ion Yield and Appearance Irradiance

3.6. High Irradiance Limit of the Ion Yield and Saturation Irradiance .43

3.7 Integrating the Other Rate Laws ............................................................. 45

3.8 Characteristics of Sequential Tunneling …….......................................54

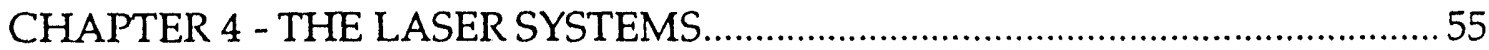

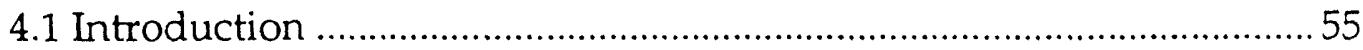

4.2 Description of Laser Pulses and Irradiance …………………................5

4.3 The Colliding Pulse Mode-Locked Dye Laser System...........................58

4.4 Autocorrelation Measurement of the CPM Pulse Width. .....................60 
5.1 Introduction .74

5.2 The Ion Spectrometer. 77

5.3 The Microchannel Plate Detector 85

5.4 Data Acquisition 87

5.5 Summary 88

CHAPTER 6 - 614NM IONIZATION DATA AND NONSEQUENTIAL IONIZATION.

6.1 Introduction 89

6.2 Linear Polarization Data at $614 \mathrm{~nm}$ 89

6.4 Resonance Effects 93

6.4 Nonsequential Ionization 94

6.5 Circular Polarization Data at $614 \mathrm{~nm}$ 102 
6.6 Summary

Chapter 7-800NM IONIZATION DATA …………................................................. 106

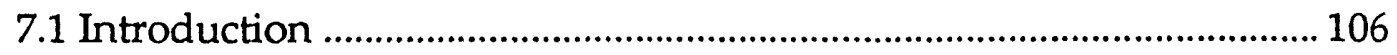

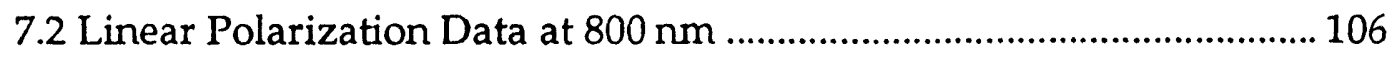

7.3 Circular Polarization Data at $800 \mathrm{~nm}$...............................................112

7.4 Data for Other Ellipticities at $800 \mathrm{~nm}$..................................................... 114

7.5 Wavelength Comparison .................................................................... 118

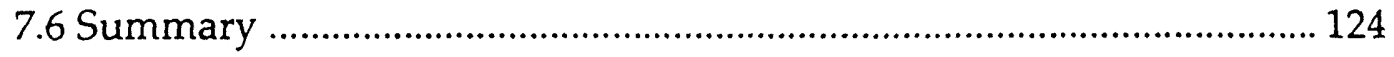

CHAPTER 8-THE SPECIES DEPENDENCE OF SEQUENTIAL

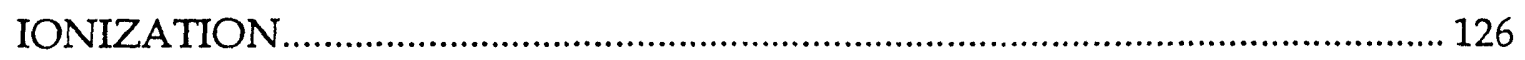

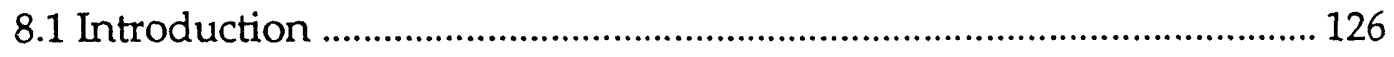

8.2 Appearance Irradiances at $800 \mathrm{~nm}$ with Circular Polarization ............ 126

8.3 Discussion of Barrier Suppression Ionization Description ................... 127

8.4 Species Dependence of the ADK Model ................................................ 129

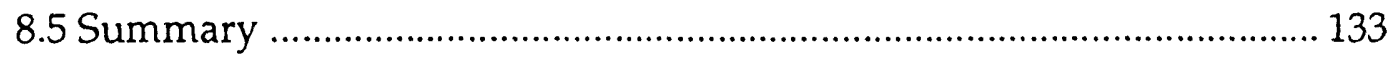

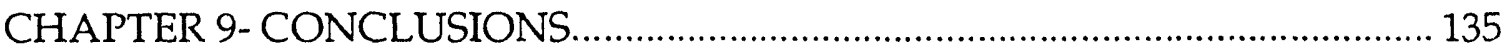

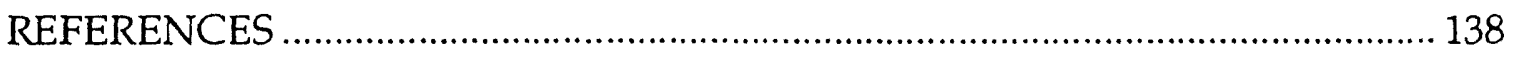

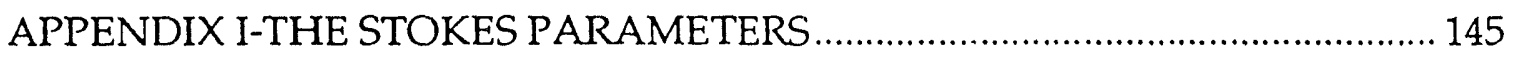




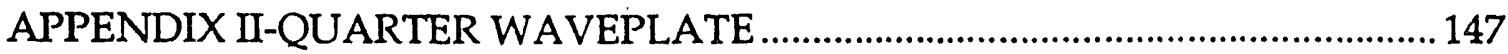

APPENDIX III-THE PONDERMOTIVE POTENTIAL ............................................ 151

APPENDIX IV-THE ATOMIC UNIT OF IRRADIANCE …………...................... 154 


\section{LIST OF TABLES}

2.8.1 Factors to convert atomic units to common experimental units.

3.3.1 Argon threshold and saturation irradiances for the simple atom model for circular polarization

3.7.1. Scaling Laws and formulas for the simple-atom model for circular polarization. 48

3.7.2. Scaling Laws and formulas for the cycle-averaged simple atom model for linear polarization.

3.7.3. Scaling Laws and formulas for the complex-atorn or ADK model for circular polarization. 50

3.7.4. Scaling Laws and formulas for the cycle-averaged complex atom or ADK model for linear polarization.

3.7.5. Scaling Laws and formulas for the multiphoton model. $\mathrm{n}$ is the number of photons required to ionize the atom. 52

3.7.6 The ADK threshold and saturation irradiances for linear and circular polarization

7.5.1 The measured 0.1 ion appearance irradiances for linear polarization for the doubly ionized charge states and the corresponding calculated nonsequential threshold irradiances.

8.4.1 The effective principle quantum numbers for the neutral and singly ionized ions of helium, neon and argon. 


\section{LIST OF FIGURES}

2.2.1 MPI scheme for adding photon energies to reach the continuum. 7

2.3.1 The unperturbed atomic potential well binds the electron to the atom.

2.3.2 The applied field alters the potential well allowing the electron to tunnel out.

2.4.1 The ADK (solid lines) and SAE (dashed lines) predictions for the He ionization rates for linear polarization at $616 \mathrm{~nm}$

2.6.1 The ADK (solid lines) and SAE (dashed lines) predictions for the He ion yields.

3.2.1 The plot of the numerical integration of Equation 3.2.1

3.2.2 The numerically calculated ion balance of the charge states of argon.

3.2.3 The closed form solution for the ion balance of the charge states of argon

3.3.1 A plot of the total area under the rate curve as a function of irradiance.

3.4.1 The spatial distributions of ions produced in a Gaussian beam.

3.5.1 The low irradiance limit of the density normalized ion yield is compared to the numerically calculated results. 
3.5.2 The simple atom model appearance irradiance for $\mathrm{Ar}+1$ as a function of irradiance at three densities for circular polarization

3.6.1 The high irradiance limit of the density normalized ion yield is compared to the numerically calculated results.

4.3.1 The colliding-pulse mode-locked dye laser system. 58

4.4.1 Single-shot autocorrelator uses nonlinear second harmonic generation to monitor pulse width.

4.4.2 Single-single shot autocorrelation and a fitted autocorrelation for a hyperbolic-secant-squared pulse.

4.5.1 Initial profile at the focus of the $\mathrm{f} / 5$ off-axis parabola.

4.5.2 Initial profile at the focus of the $\mathrm{f} / 5$ off-axis parabola viewed on a logarithmic scale. 64

4.5.3 Final profile at the focus of the $\mathrm{f} / 5$ off-axis parabola at $614 \mathrm{~nm}$.

4.5.4 Final profile at the focus of the $\mathrm{f} / 5$ off-axis parabola at $614 \mathrm{~nm}$ viewed on a logarithmic scale.

4.6.1 Typical CPM laser system spectrum 67

4.6.2 Spectrum of the CPM-laser pulses after installation of thick BK-7 lenses showing strong self-phase modulation.

4.8.1 The titanium sapphire laser system 70

4.8.2 The autocorrelation of the 800-nm laser pulse. 71 
4.8.3 Focal profile of the $800-\mathrm{nm}$ laser pulse using the $10 \mathrm{~cm}$ focal length parabola.

4.8.4 Logarithm of the focal profile at the focus of the 800-nm laser pulse using the $10 \mathrm{~cm}$ focal length parabola. 72

5.1.1 Diagram of the ion yield experiment. 75

5.2.1 Ion Time-of-Flight Tube. 77

5.2.2 The measured flight time as a function of mass-to-charge for several ions.

5.2.3 The spectrometer resolution 81

5.2.4 The ion signal as a function of time for several different pressures 83

5.2.5 The pressure dependence of the ion yields using the $800 \mathrm{~nm}$ laser. 84

5.3.1 Schematic of the ion detector. 85

5.3.2 Results of the pulse height analysis of several thousand data shots 86

6.2.1 Helium and neon ion yields for 120-fs linearly polarized pulses at 614 nm. 90

6.2.2 The helium ion yields superimposed on the yields predicted by SAE theory. 91

6.2.3 The singly and doubly charged helium and neon ion yields superimposed on the predictions for sequential ADK theory..... 92 
6.4.1 The data for linearly polarized $614-\mathrm{nm}$ pulses with a shake-off prediction(DIR) for helium and neon.

6.4.2 The 614-nm helium data for linear polarization plotted with the results of a rescattering calculation for $0.6 \mu \mathrm{m}$

6.4.3 The 614-nm neon data for linear polarization plotted with the results of a rescattering calculation for $625-\mathrm{nm} 100-\mathrm{fs}$ pulses

6.4.4 The $\mathrm{He}^{+2}$ and $\mathrm{Ne}^{+2}$ ion yields for linear polarization where the irradiances of the data have been scaled to align the sequential portion of the curves.

6.5.1 The helium and neon ion yields produced by circularly polarized $120-\mathrm{fs}$ laser pulses at $614 \mathrm{~nm}$. The ADK threshold and saturation irradiances are listed in Table 3.7.6 for comparison

6.5.2 The direct calculation(DIR) of the ion yields with the 614-nm data for circular polarization

6.5.3 The 614-nm data for circular polarization and the corresponding ADK predictions.

7.2.1 The helium, neon and argon ion yields produced by linearly polarized $130-$ fs laser pulses at $800 \mathrm{~nm}$ 107

7.2.2 The helium and neon ion yields produced by linearly polarized 130fs laser pulses at $800 \mathrm{~nm}$ superimposed on the sequential ADK predictions. The ADK threshold and saturation irradiances are listed in Table 3.7.6 for comparison. 
7.2.3 The argon ion yields produced by linearly polarized 130-fs laser pulses at $800 \mathrm{~nm}$ superimposed on the sequential ADK predictions.

7.2.4 The neon ion yields produced by linearly polarized 130-fs laser pulses at $800 \mathrm{~nm}$ superimposed on the predictions of the ADK model including rescattering of the first electron removed.

7.2.5 The irradiance normalized ion yields for $\mathrm{He}+2$ and $\mathrm{Ne}+2$ for linear polarization at $800 \mathrm{~nm}$

7.3.1 The helium, neon and argon ion yields produced by circularly polarized 130-fs laser pulses at $800 \mathrm{~nm}$

7.3.2 The helium and neon ion yields produced by circularly polarized 130-fs laser pulses at $800 \mathrm{~nm}$ superimposed on the sequential ADK predictions.

7.3.3 The argon ion yields produced by circularly polarized 130-fs laser pulses at $800 \mathrm{~nm}$ superimposed on the sequential ADK predictions.

7.4.1 The doubly ionized helium yield produced by $130 \mathrm{fs}$ pulses at $800 \mathrm{~nm}$ with ellipticities of $e=0.0,0.2,0.4$, and 1.0 .

7.4.2 The doubly ionized helium yield as a function of ellipticity superimposed on the ADK predictions 116

7.4.3 The $\mathrm{He}+2$ yield data at $800 \mathrm{~nm}$ versus ellipticity plotted with a rescattering prediction for $\mathrm{Ne}+2$ at $625 \mathrm{~nm}$. The data is for $2.0 \times 1015$ $\mathrm{W} / \mathrm{cm} 2$ and linear polarization. The rescattering calculation is for $100 \mathrm{fs}$ pulses at irradiances of $9 \times 1014 \mathrm{~W} / \mathrm{cm} 2$ 
7.5.1 The ADK He ion yields for $614 \mathrm{~nm}$ and $800 \mathrm{~nm}$ scaled to remove the differences in characteristic focal volume. The theory is for linear polarization.

7.5.2 The helium ion yields for linear polarization at $614 \mathrm{~nm}$ and $800 \mathrm{~nm}$ scaled to the singly ionized charge states

7.5.3 The neon ion yields for $614 \mathrm{~nm}$ and $800 \mathrm{~nm}$ scaled to the singly ionized charge states

7.5.4 Comparison of the 614 and $800 \mathrm{~nm}$ single ion appearance irradiances for helium and neon.

8.2.1 The single ion appearance irradiance as a function of ionization potential for singly and doubly charged states of $\mathrm{He}, \mathrm{Ne}$ and $\mathrm{Ar}$ produced by circularly polarized pulses at $800 \mathrm{~nm}$.

8.4.1 The appearance irradiance from the ADK model for circular polarization plotted versus ionization potential for $n=1$ to 5 .

8.4.2 The ADK model appearance irradiances as a function of ionization potential for $\mathrm{He}, \mathrm{Ne}$ and $\mathrm{Ar}$ produced by circularly polarized pulses at 800 $\mathrm{nm}$.

8.4.3 The measured appearance irradiances with a cubic power fit in the ionization potential.

A.II.1 The ellipticity of the output wave for a linearly polarized wave incident perpendicular to the optic axis of a uniaxial-crystal quarter-wave retarder. 


\begin{abstract}
This dissertation research is an investigation of the strong optical field ionization of atoms and ions by 120-fs, 614-nm laser pulses and 130-fs, 800-nm laser pulses. The experiments have shown ionization that is enhanced above the predictions of sequential tunneling models for $\mathrm{He}^{+2}, \mathrm{Ne}^{+2}$ and $\mathrm{Ar}^{+2}$. The ion yields for $\mathrm{He}^{+1}$, $\mathrm{Ne}^{+1}$ and $\mathrm{Ar}^{+1}$ agree well with the theoretical predictions of optical tunneling models. Investigation of the polarization dependence of the ionization indicates that the enhancements are consistent with a nonsequential ionization mechanism in which the linearly polarized field drives the electron wavefunction back toward the ion core and causes double ionization through inelastic e-2e scattering. These investigations have initiated a number of other studies by other groups and are of current scientific interest in the fields of high-irradiance lasermatter interactions and production of high-density plasmas. This work involved-
\end{abstract}

1. Understanding the characteristic nature of the ion yields produced by tunneling ionization through investigation of analytic solutions for tunneling at optical frequencies.

2. Extensive characterization of the pulses produced by $614-\mathrm{nm}$ and $800-\mathrm{nm}$ ultrashort pulse lasers. Absolute calibration of the irradiance scale produced shows the practicality of the inverse problem-measuring peak laser irradiance using ion yields.

3. Measuring the ion yields (number of ions produced versus irradiance) for three noble gases using linear, circular and elliptical polarizations of laser pulses 
at $614-\mathrm{nm}$ and $800-\mathrm{nm}$. The measurements are some of the first measurements for pulse widths as low as 120-fs. 


\section{CHAPTER 1 - INTRODUCTION}

\subsection{Preface}

As early as 1928 Oppenheimer discussed the semiclassical ionization of hydrogen by electric fields[1]. In the semiclassical approach, the photon interactions are treated as an applied field that suppresses the atomic potential and allows the electron to tunnel through the Coulomb barrier. While low-frequency field ionization experiments have been possible on high-lying states of atoms[2-4], producing fields at optical frequencies that are large enough to field ionize the tightly bound electrons in ground state or singly charged noble gases was not possible until the creation of modern ultrashort-pulse lasers. Experiments on optical field ionization have followed the development of ultrashort pulse lasers from tens of picoseconds to 0.5 picoseconds [5-11]. Multiphoton ionization experiments have been performed for pulse widths as short as 22 femtosecond[12-16]. In this work 120-fs, 614-nm and 130-fs, 800-nm laser pulses with millijoule energies have permitted experiments on the field ionization of helium and neon[17-19]. These experiments agree well with tunneling theory for removal of one electron. The measured ion yields versus irradiance for the doubly charged ions exhibit sequential field ionization combined with nonsequential ionization.

A fundamental understanding of optical field ionization (OFI) using intense ultrashort laser pulses can generally be applied to the study of tunnel-ionized plasmas, plasma mirrors, laser pulse propagation, recombination X-ray/XUV laser development and the determination of peak laser irradiances. The experiments are also valuable in understanding electron correlations in multielectron atoms. 
In this dissertation I will discuss the theoretical and experimental issues of nonsequential and sequential ionization in helium, neon and argon by ultrashort laser pulses at $614 \mathrm{~nm}$ and $800 \mathrm{~nm}$. I will specifically discuss two possible nonsequential ionization mechanisms, the polarization dependence of the ionization and scaling relationships for wavelength and species.

\subsection{Outline}

The format of this dissertation is as follows: Chapter 2 is a discussion of the basic concepts of optical field ionization, outlining the differences between multiphoton ionization and tunneling ionization and between sequential and nonsequential ionization. The chapter also explains focal volume effects on the overall ion yield versus irradiance. Chapter 3 describes analytic solutions to the sequential rate equations for several tunneling and multiphoton models, emphasizing that the ion yields for sequential ionization are described by characteristic curves regardless of the charge state of the species being ionized. Violation of this characteristic behavior indicates a violation of the tunneling or sequential assumptions. Chapters 4 and 5 describe the laser systems and the experimental configuration for the ion yield measurements. It includes discussions of the description of the laser pulses, the resolution and efficiency of the time-of flight spectrometer used to count the ions and space-charge effects in the spectrometer. Chapter 6 presents the He and Ne data for 614-nm with linear polarization and discusses the deviations observed from sequential tunneling ionization. Two possible nonsequential mechanisms are presented. The first mechanism is shake-off model based on a sudden shock to the ion. The second mechanism is a rescattering mechanism in which the linearly polarized field drives the electron wavefunction back toward the ion core and causes double ionization through inelastic scattering. I then discuss the mechanisms' 
predictions for circular polarization and present data supporting the rescattering mechanism as the cause of the nonsequential ionization. Chapter 7 presents data on $\mathrm{He}, \mathrm{Ne}$ and $\mathrm{Ar}$ for $800 \mathrm{~nm}$ that supports the conclusions of the Chapter 6 . Chapter 7 also provides more extensive examination of the polarization dependence of the ionization and the wavelength dependence of the appearance irradiance. Chapter 8 discusses the species dependence of sequential ionization. Finally, Chapter 9 presents a summary of the conclusions of this work. 


\section{CHAPTER 2 - AN INTRODUCTION TO OPTICAL FIELD IONIZATION}

\subsection{Introduction}

For an atom in a radiation field, the time-dependent Schrödinger equation governing the evolution of the system is

$$
i \hbar \frac{\partial \Psi(r, t)}{\partial t}=H \Psi(r, t)
$$

Here the total Hamiltonian, $H$, may be written $[20,21]$

$$
H=H_{\text {rad }}+H_{\text {atom }}+H_{\text {int }}
$$

In this expression $H_{\text {rad }}$ describes all the modes $k \lambda$ of the radiation field

$$
H_{r a d}=\sum_{k \lambda} \hbar \omega_{k}\left(a_{k \lambda}^{+} a_{k \lambda}+\frac{1}{2}\right)
$$

The atomic Hamiltonian,

$$
H_{\text {atom }}=\sum_{i}\left(\frac{p_{i}^{2}}{2 m}\right)+V
$$

defines the atomic state. The Hamiltonian, $H_{i n t}$, describes the interaction between the field and the atom including the scalar potential energy, kinetic energy and interaction terms responsible for absorption, emission, scattering and the Zeeman effect, terms for relativistic energy corrections, the spin-orbit interaction and the Darwin term. When discussing the theory of laser ionization of atoms, the method used to solve the time-dependent Schrödinger equation will often determine the way the ionization process is viewed. 


\subsection{Multiphoton Ionization}

Multiphoton ionization is the best known description of the ionization of an atom in a laser field[22]. The multiphoton ionization(MPI) description applies when perturbation theory may be used to consider the ionization of the atom by the absorption of many photons. Figure 2.2.1 shows the physical scheme for absorbing multiple photons through a series of virtual states to reach the continuum.

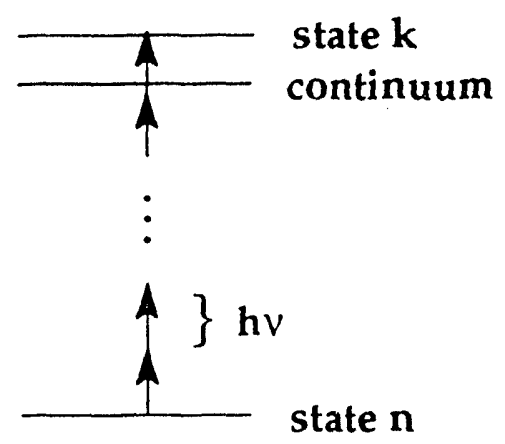

Figure 2.2.1 MPI scheme for adding photon energies to reach the continuum.

Using propagator techniques[23] the transition rate from an initial state $n$ to final states $k$ with density of states $\rho_{k}$ for a Kth-order process is

$$
W_{k n}^{(K)}=2 \pi\left|z_{k n}^{(K)}\right|^{2} F_{0}^{2 K} \rho_{k}
$$

Here $F_{0}$ represents the static electric field. The $K$ th-order coefficient, $z_{k n}^{(K)}$, is defined using the $K$ th-order matrix elements 


$$
V_{k n}^{(K)}=\frac{1}{2^{K}} \sum_{m s \cdots p} \frac{V_{k m}^{(1)} V_{m s}^{(1)} \cdots V_{p n}^{(1)}}{\left[\omega_{m n}-(K-1) \omega\right] \cdots\left[\omega_{p n}-\omega\right]} \equiv z_{k n}^{(K)} F_{0}{ }^{K}
$$

where

$$
z_{k n}^{(K)}=\sum_{m s \cdots p} \frac{z_{k m}^{(1)} z_{m s}^{(1)} \cdots z_{p n}^{(1)}}{\left[\omega_{m n}-(K-1) \omega\right] \cdot \cdot\left[\omega_{p n}-\omega\right]}
$$

In general, experimenters cast the rate in the form

$$
W_{k n}=\sigma_{k n}^{(K)}(\omega) I^{K}(r, \omega, t)
$$

Here $K$ is the minimum number of photons required for ionization from the initial state, $n$, to the final continuum state, $k . I$ is the irradiance. The $K$ th-order cross section, $\sigma_{k n}^{(K)}$, has $2^{K}$ terms. Due to the difficulty of calculating the cross section for large $K$, experimenters often treat the cross section as an experimentally determined parameter[12].

Several physical criteria determine the applicability of Kth-order perturbation theory. The value of the matrix elements can change significantly if a resonance of multiplicity $K$ or less exists between the initial, final and intermediate states. The condition for a resonance is

$$
\left|\omega_{k n} \pm K \omega\right|>>z_{k n} F_{0}
$$

Also the matrix elements must be small compared to the differences of the energies of the unperturbed system and to the energy of the photons:

$$
\frac{z_{k n} F_{0}}{\omega_{k n}}<<1 \text { and } \frac{z_{k n} F_{0}}{\omega}<<1
$$

For these experiments the electric fields can be of order one atomic unit of field, $5.14 \times 10^{9} \mathrm{~V} / \mathrm{cm}$, and the photon energies are $5.70 \times 10^{-2}$ and $7.42 \times 10^{-2}$ atomic units. 
Thus the experiment is geherally out of the region of validity for perturbation theory, and it is essential to consider strong field, nonperturbative theories..

\subsection{Tunneling Ionization}

In the tunneling ionization description, an applied electric field suppresses the atomic potential asymmetrically. The electron then has finite probability to tunnel through the barrier and escape from the atom. Figures 2.3.1 and 2.3.2 illustrate this idea. In Figure 2.3.1 the atomic potential well binds the electron to the atom, but in Figure 2.3.2 the application of an electric field suppresses the atomic potential allowing the electron to tunnel through the barrier. Solving the Schrödinger equation for the system then reduces to the case of quantum tunneling through the suppressed potential. For applied fields at optical frequencies the applied field oscillates at a high rate and tunneling occurs on a time scale short compared to the laser pulse.

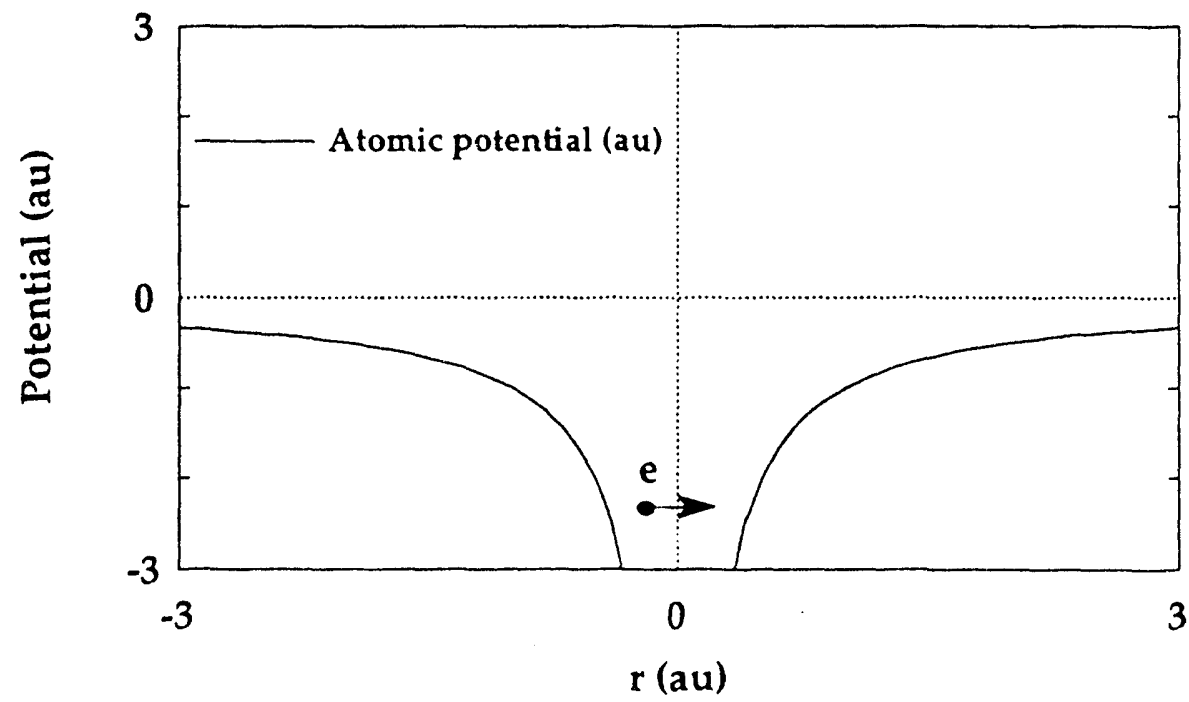

Figure 2.3.1 The unperturbed atomic potential well binds the electron to the atom. The example is hydrogen. 


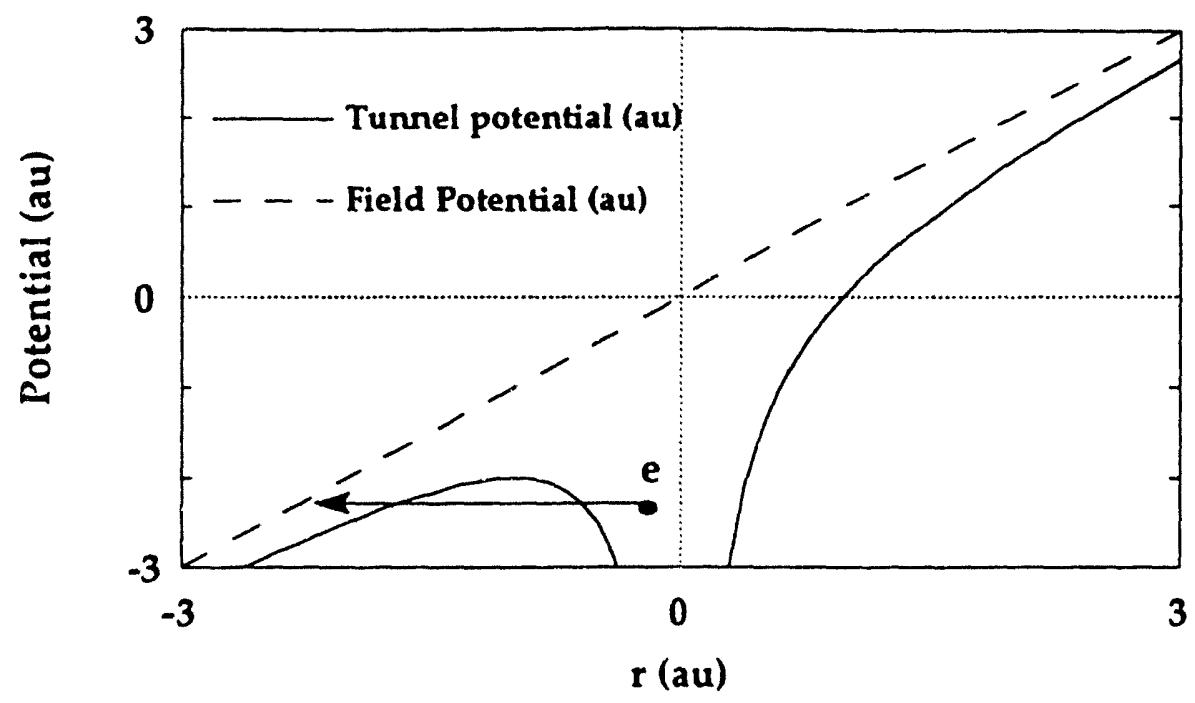

Figure 2.3.2 The applied field alters the potential well allowing the electron to tunnel out. The example is hydrogen with an applied field of one atomic unit.

For hydrogenic atoms in a static field, $F_{0}$, in atomic units the well-known 3dimensional tunneling solution[24] gives the ionization rate

$$
\Gamma=6 \omega_{0}(2 E)\left(\frac{\frac{2}{3}(2 E)^{3 / 2}}{F_{0}}\right) \exp \left(-\frac{\frac{2}{3}(2 E)^{3 / 2}}{F_{0}}\right)
$$

Here $E$ is the ionization potential in atomic units, and $\omega_{0}$ is the atomic unit of frequency (With $\omega_{0}=4.1 \times 10^{16} \mathrm{sec}^{-1}$ the result is in sec- 1 . With $\omega_{0}=1$ au the result is in atomic units). Gibson[10]et al. call this the static simple atom case.

Keldysh[25] solved the Schrödinger equation for tunneling ionization in a strong electromagnetic field with photon energy lower than the ionization potential. For an electron with ionization potential $E$, the ionization rate is 


$$
\Gamma=A \omega\left(\frac{E}{\hbar \omega}\right)^{3 / 2}\left(\frac{\gamma}{\left(1+\gamma^{2}\right)^{1 / 2}}\right)^{5 / 2} S\left(\gamma, \frac{\bar{E}}{\hbar \omega}\right) \exp \left\{-\frac{\bar{E}}{\hbar \omega}\left[\sinh ^{-1} \gamma-\gamma \frac{\left(1+\gamma^{2}\right)^{1 / 2}}{1+2 \gamma^{2}}\right]\right\}
$$

The Keldysh parameter, $\gamma$, is

$$
\gamma=\omega / \omega_{1}=\omega(2 m E)^{4 / 2} / e F_{0}
$$

where $\omega_{t}$ is the tunneling rate. The effective ionization potential, which incorporates the strong field shift, is

$$
\bar{E}=E+e^{2} F_{0}^{2} / 4 m \omega^{2}=E\left(1+1 / 2 \gamma^{2}\right)
$$

$A$ is a numerical coefficient. The function $S(\gamma, \bar{E} / \hbar \omega)$ varies slowly with the frequency and field. The quantity $e^{2} F_{0}^{2} / 4 m \omega^{2}$ is called the pondermotive potential and is discussed in Appendix III. The Keldysh parameter can be used as a benchmark to differentiate between the multiphoton case and the tunneling case. When $\omega \gg \omega_{t}, \gamma \gg 1$, and the rate formula describes multiphoton absorption. For low frequencies or strong fields, when $\omega<\omega_{\imath}, \gamma<<1$, the rate exhibits the exponential dependence on the ionization potential and the external electric field strength, $F_{0}$, that characterizes the static rate in Equation (2.3.1):

$$
\Gamma \propto \exp \left[-\frac{2(2 E)^{3 / 2}}{3 F_{0}}\right]
$$

Using time dependent perturbation theory Oppenheimer[1] obtained this factor in the rate for ionization of hydrogen by low static fields. The Keldysh rate does not reproduce the coefficient before the exponential in the static tunneling rate for hydrogen[24] for low frequencies. The condition $\omega<\omega_{t}$ for tunneling may 
be restated to show directly the relationship between $\omega$ and $F_{0}$ that is necessary for tunneling to occur. That is

$$
\frac{\omega}{F_{0}} \ll \frac{1187 \times 10^{-4}}{\sqrt{E}} \text { (MKS units). }
$$

In terms of the wavelength and units appropriate to laser experiments

$$
F_{0}(\mathrm{~V} / \mathrm{cm}) \lambda(\mu \mathrm{m})>>6.35 \times 10^{7} \sqrt{E(\mathrm{eV})}
$$

This shows that tunneling at optical wavelengths from the tightly bound ground states of noble gases requires reaching electric field strengths on the order of an atomic unit of field $\left(5.142 \times 10^{9} \mathrm{~V} / \mathrm{cm}\right)$ before ionization occurs. Thus studying optical field ionization requires ultrashort high-irradiance pulses.

While Sauer et. al.[4] correctly point out that the condition $\gamma<1$ cannot strictly be considered to define the tunneling regime, tunneling models have also been shown to be predictive in this regime $[8,10,26]$. In the experiments discussed in this dissertation, $\gamma$ varies from 0.4 to 1.0 .

Perelomov, Popov, and Terent'ev[27-30] solved the Schrödinger equation for the ionization of nonhydrogenic atoms and elliptical polarizations. Using the coefficients of Ammosov, Delone and Krainov[31] the rate is

$$
\Gamma=\left(\frac{|\varepsilon|(1+|\varepsilon|)}{2}\right)^{-1 / 2} a\left(\frac{1-|\varepsilon|}{3|\varepsilon|} \frac{(2 E)^{3 / 2}}{\mathrm{~F}_{0}}\right) \Gamma_{\text {stat }}\left(F_{0}\right) .
$$

Here the field of the elliptically polarized wave with temporal envelope $F_{0}(t)$ is given by

$$
F(t)=F_{0}(t)\left(e_{x} \cos \omega t+\varepsilon e_{y} \sin \omega t\right),
$$

and 


$$
a(x)=e^{-x} I_{0}(x)
$$

$I_{0}(x)$ is a Bessel function of imaginary argument, and $\Gamma_{\text {sat }}$ is the ionization rate in a static field

$$
\Gamma_{\text {stat }}\left(F_{0}\right)=C_{n^{\prime} \cdot t^{2}}^{2} E \frac{(2 l+1)(l+|m|) !}{2^{|m|}(|m|) !(l-|m|) !}\left(\frac{2(2 E)^{3 / 2}}{F_{0}}\right)^{2 n^{\circ}-|m|-1} \exp \left(-\frac{2(2 E)^{3 / 2}}{3 F_{0}}\right) .
$$

$n^{*}$ is the effective principle quantum number. In atomic units

$$
n^{*}=Z(2 E)^{-1 / 2}
$$

$Z$ is the charge of the atomic residue. The effective orbital quantum number is defined as

$$
l^{*}=n_{0}^{*}-1
$$

Here $n_{0}{ }^{*}$ is the effective principle quantum number of the lowest state with the given quantum number $l$. The original paper[31] incorrectly defines $n_{0}{ }^{*}$ as the effective principle quantum number of the ground state[32]. This is only true if $l$ is the ground state quantum number. The factor $C_{n * \%}^{2}$ is given by

$$
C_{n^{* *}}^{2}=\frac{2^{2 n^{*}}}{n^{*} \Gamma\left(n^{*}+l^{*}+1\right) \Gamma\left(n^{*}-l^{*}\right)^{\prime}}
$$

where $\Gamma(x)$ is the gamma function of $x$. This model is referred to as the ADK or (in reference [10]) the complex atom model.

Note that in the case of circular polarization the complex atom tunneling rate given in (2.3.8) has been shown to reduce to

$$
\Gamma=\Gamma_{\text {stat }}
$$


as expected since the magnitude of a the electric field in a circularly polarized wave is constant over time. For linear polarization the rate reduces to the cycle averaged rate as well[27].

Augst et al. $[8,9]$ argued that a one dimensional, purely classical Barrier Suppression Ionization(BSI) description should predict the ionization. While the model predicts ionization thresholds reasonably well for 1-ps pulses due to a cancellation of defects[10], BSI does not allow prediction of the actual ionization rates and is unphysically independent of the laser pulse width. This description is discus $\mathrm{d}$ further in Chapter 8 in connection with the species dependence of sequential ionization.

\subsection{The Single Active Electron Model}

The single-active-electron model(SAE) [33-37] explicitly follows the time evolution of a valence electron in the electromagnetic field of the laser pulse and the frozen mean-field of the remaining electrons and the nucleus. The model is expected to be accurate for rare gas atoms, at least partially because the neglected double or higher excitations involve states well above the single ionization threshold[34].

In the SAE model, the Hamiltonian is explicitly time-dependent.

$$
H(t)=H_{0}+V_{l}(t)
$$

Here $H_{0}$ is the non-relativistic Hamiltonian of the atom. $V_{l}(t)$ describes the interaction of the electrons with the laser field. In the model, the laser polarization is linear and oriented along the $\mathbf{z}$-axis. Since the applied laser fields are of the order of the atomic fields, the model treats them semi-classically. Thus 


$$
V_{1}(t)=-e \sum_{i} z_{i} F_{0}(t) \sin (\omega t)
$$

Here $F_{0}(t)$ is the pulse envelope, and $i$ indicates summation over the electrons at positions $z_{i}$. Present computational techniques are incapable of solving the full multielectron problem. Thus the SAE model relies on the Hartree-Fock method that treats the time-dependent wavefunction as a product of single particle orbitals. The model can account for single-electron resonance effects, but it cannot account for effects due to double-electron excited levels. As the actual calculations of the SAE model were not part of this work, I will not further describe the numerical techniques used to solve the Schrödinger equation. A comparison, however, between the ADK and SAE predictions for the integrated ionization rates as a function of irradiance is useful as a cross check of the two models. Figure 2.4.1 shows the excellent agreement between the helium ADK

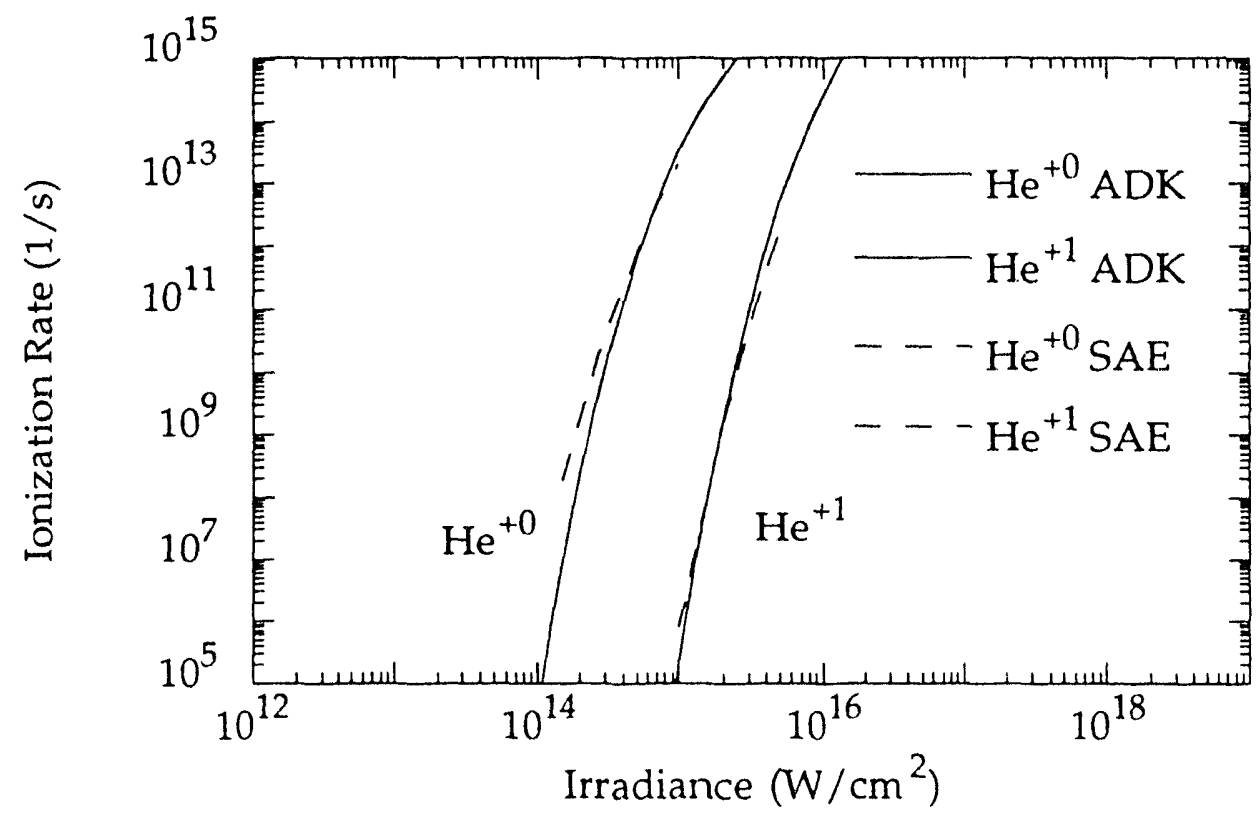

Figure 2.4.1 The ADK (solid lines) and SAE (dashed lines) predictions for the He ionization rates for linear polarization at 616 nm. (SAE rates courtesy of Ken Kulander). 
and SAE ionization rates as a function of irradiance for linear polarization at 616 nm.

\subsection{The Rate Equations}

Since target gas pressures in these experiments are well below $10 \mathrm{~Pa}$, recombination and collisional ionization occur on a time scale much greater than the time scales of the experiment and are unimportant (The proton collision fiequency for Coulomb collisions is on the order of $40 \mathrm{~s}^{-1}$ [38]). At the outset of this work little evidence existed for nonsequential processes in which two or more electrons are removed from bound states at the same irradiance in the pulse $[7,10,12,15,16,39,40]$. Under collisionless conditions the rate equations for sequential ionization are:

$$
\left.\begin{array}{rl}
\frac{d p_{0}}{d t} & =-\Gamma_{0} p_{0} \\
\frac{d p_{1}}{d t} & =-\Gamma_{1} p_{1}+\Gamma_{0} p_{0} \\
& \cdots \\
\frac{d p_{k}}{d t} & =-\Gamma_{k} p_{k}+\Gamma_{k-1} p_{k-1} \\
& \ldots \\
\frac{d p_{n}}{d t} & =\Gamma_{n-1} p_{n-1}
\end{array}\right\}
$$

where $p_{k}$ is the probability that the atom is $k$ times ionized. The $\Gamma_{k}$ are the ionization rates for charge state $k$ and depend implicitly on time and position.

For this work, I use the backward difference method to solve the sequential rate equations. For purely sequential ionization, accurate analytic approximations are given in Chapter 3. I will use the analytic solutions to discuss 
the scaling and behavior of the sequential yields. In this dissertation I use numerical integration of the rate equations wherever I compare experimental data to theory unless otherwise specified.

When considering nonsequential ionization of the outer two electrons in a multi-electron atom, the populations can be represented by the following coupled set of rate equations:

$$
\left.\begin{array}{l}
\frac{d p_{0}}{d t}=-\Gamma_{0} p_{0} \\
\frac{d p_{1}}{d t}=-\Gamma_{1} p_{1}+\Gamma_{0}^{\prime} p_{0} \\
\frac{d p_{2}}{d t}=\left(\Gamma_{0}-\Gamma_{0}^{\prime}\right) p_{0}+\Gamma_{1} p_{1}
\end{array}\right\}
$$

If the ionization process is purely sequential, $\Gamma_{0}^{\prime}$ will exactly equal $\Gamma_{0}$, and the equations revert to Equations (2.5.1). If any process exists that transfers population directly from the neutral to the double ion, then $\Gamma_{0}^{\prime}<\Gamma_{0}$. In this case the appearance irradiance for the second ion will be lower than the sequential appearance irradiance. It is, therefore, critical to predict sequential appearance irradiances accurately. If a direct component becomes possible at high irradiance, it will be measurable only if the pulse is short enough. At long pulse widths the neutral population can deplete before the irradiance reaches the threshold for the direct process, and at for high applied fields neutral population must still exist within the focal volume for a direct process to occur. This emphasizes the need to use ultrashort pulses in studying tunneling or optical field ionization. Thus any direct component will volume saturate in parallel with the single ion signal. The 
physics of the direct double ionization process will define the magnitude and irradiance dependence of these rate constants and determine the exact nature of the photoelectron spectrum.

\subsection{The Focal Volume}

When measuring the number of ions produced in a laser focus, one must take into account the irradiance profile of the beam. As noted before, the ionization rates are extremely strong functions of the irradiance. Thus the ionization exhibits a threshold behavior as a function of irradiance. The shape and size of the focal volume bounded by this threshold irradiance will largely determine the actual number of ions observed. For the 614-nm pulses used in the experiment we assume that the laser irradiance is a focused beam with a Gaussian spatial profile and a hyperbolic-secant- $s q u a r e d$ temporal profile. This assumption is consistent with experimental measurements of the pulse parameters that are discussed more fully in Chapter 4. The envelope of the electric field is then

$$
F_{0}(r, t)=\frac{\mathcal{F}_{0}}{\sqrt{1+\left(\frac{\lambda z}{\pi w_{0}^{2}}\right)^{2}}} \exp \left(-\frac{\frac{r^{2}}{w_{0}^{2}}}{1+\left(\frac{\lambda z}{\pi w_{0}^{2}}\right)^{2}}\right) \operatorname{sech}\left(1.76 \frac{t}{\tau}\right)
$$

Here $\mathcal{F}_{0}$ is the peak strength of the electric field, $\lambda$ is the laser wavelength, $w_{0}$ is the $1 / \mathrm{e}^{2}$ radius of the focal spot, and $\tau$ is the full width at half maximum of the pulse. For the $800 \mathrm{~nm}$ pulses, I assume that the focused beam has a Gaussian spatial profile and a Gaussian temporal profile. The electric field is then 


$$
F_{0}(r, t)=\frac{\mathcal{F}_{0}}{\sqrt{1+\left(\frac{\lambda z}{\pi w_{0}^{2}}\right)^{2}}} \exp \left(-\frac{\frac{r^{2}}{w_{0}^{2}}}{1+\left(\frac{\lambda z}{\pi w_{0}^{2}}\right)^{2}}\right) \exp \left(-2 \sqrt{\ln 2} \frac{t}{\tau}\right)
$$

For a hyperbolic-secant temporal profile and Gaussian spatial profile, Figure 2.6.1 shows the ADK and SAE predictions for the mean total number of ions produced as a function of the peak irradiance of the pulse. The pulses are taken to be linearly polarized, $120 \mathrm{fs}, 614-\mathrm{nm}$ pulses with $w_{0}=3.8 \mu \mathrm{m}$. The rapid rise in the ion yields shows clearly the threshold behavior of the ionization as a function

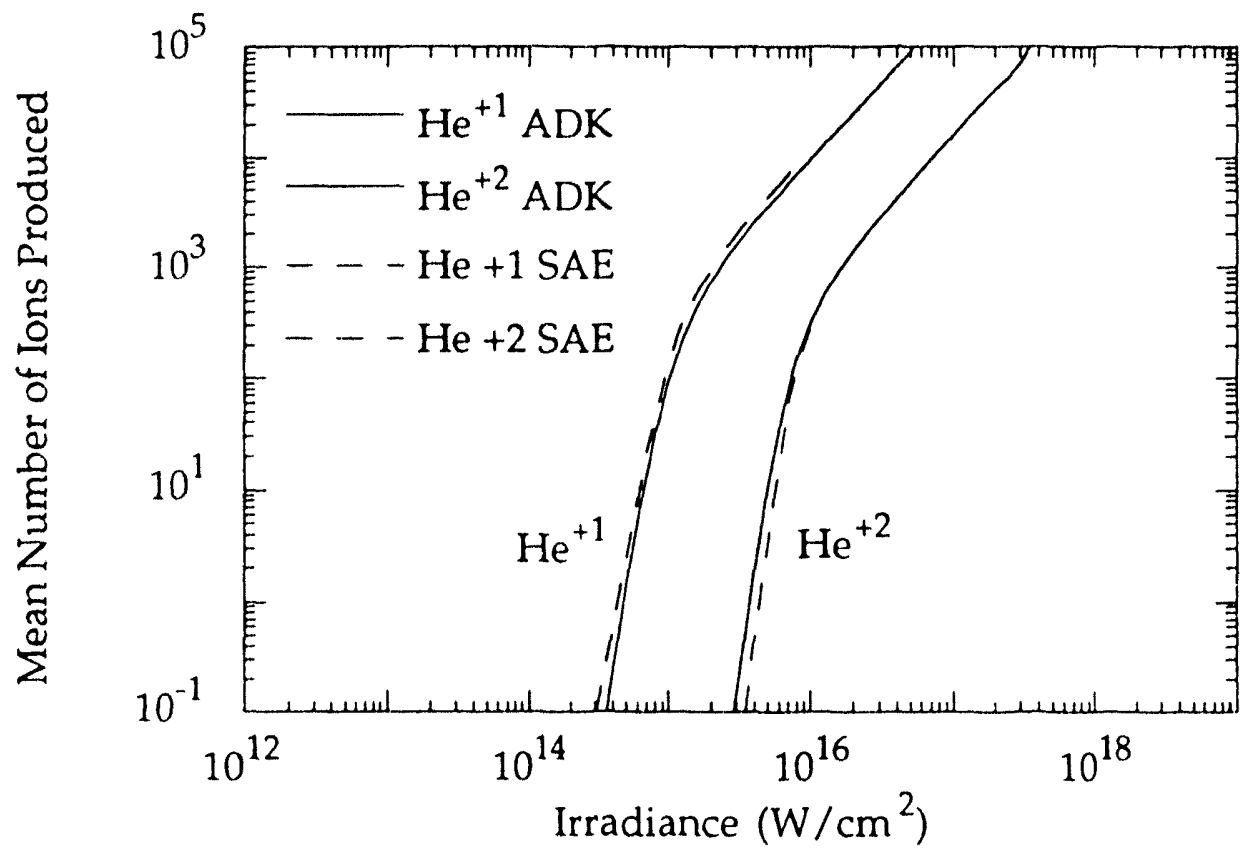

Figure 2.6.1 The ADK (solid lines) and SAE (dashed lines) predictions for the He ion yields for linearly polarized, 120fs, 614 $\mathrm{nm}$ pulses with $w_{0}=3.8 \mu \mathrm{m}$. (SAE calculations courtesy of Ken Kulander). 
of irradiance. The roll off at the top of the yield curves is due to saturation of the focal volume. Once the pulse depletes the previous charge state, the number of ions produced only increases with the increasing volume bounded by the threshold irradiance contour. The volume bounded by an iso-irradiance contour in a Gaussian focus increases as the $3 / 2$ power of the peak pulse irradiance, so in saturation the number of ions increases as the $3 / 2$ power of the peak pulse irradiance. Note also that the excellent agreement between the ADK and SAE models for the ionization rates that was shown in Figure 2.4.1 carries over to the ion yield calculation.

\subsection{Polarization Dependence of Irradiance and Units}

The polarization of the applied electromagnetic field has a strong effect on the ionization rate of an atom as shown in Equation (2.3.8). Experimentally the polarization also has a strong effect on the final ion yield versus irradiance because the irradiance of a wave depends on its polarization as well as on its peak electric field. For a low-density isotropic medium, the electric field of a wave propagating in the direction of unit vector $\mathbf{e}_{k}$ may be represented by

$$
F=F_{0}(r, t)\left[e_{i} \cos \left(\omega t-k \cdot r+\delta_{0}\right)+\varepsilon e_{j} \sin \left(\omega t-k \cdot r+\delta_{0}\right)\right] .
$$

Here $F_{0}(r, t)$ is the spatially and temporally dependent envelope function, and $\varepsilon$ is the ellipticity of the wave. $\varepsilon$ varies from -1 to 1 . For linear polarization $\varepsilon=0$. For right and left circular polarization $\varepsilon=1$ and $\varepsilon=-1$, respectively. Other ellipticities indicate that the wave is elliptically polarized. $\delta_{0}$ is a phase factor. The magnetic field of the wave is then

$$
H=\frac{1}{\mu_{0} c}\left(e_{k} \times F\right)
$$


Here I use MKS units. The Poynting vector representing the energy flow of the wave is

$$
S=F \times H .
$$

Substituting Equation (2.7.2) in Equation (2.7.3) gives

$$
S=\frac{1}{\mu_{0} c}|F|^{2} e_{k} .
$$

The irradiance, I, which is the rate that energy falls on a given area, is the time average over a cycle of the Poynting vector magnitude.

$$
I=\frac{1}{\mu_{0} c}\left\langle|F|^{2}\right\rangle
$$

Substituting Equation (2.7.1) for $F$ in Equation (2.7.5) and performing the time average gives

$$
I=\frac{1}{2 \mu_{0} c}\left(1+\varepsilon^{2}\right) F_{0}^{2} . \quad \text { (MKS units) }
$$

Thus the irradiance of the a wave with electric-field amplitude, $F_{0}$, depends on the polarization of the wave. For equal electric-field amplitudes, a circularlypolarized wave will have twice the irradiance of a linearly-polarized wave. Equivalently, we note that, for equal irradiance, the electric field amplitude of a linearly-polarized wave will be larger than the electric field amplitude of a circularly-polarized wave by the square-root of two. Since tunneling ionization has a high order dependence on the electric field, a circularly-polarized wave will have much lower ion yields than a linearly polarized wave of equal irradiance.

The majority of the theory for this work is in atomic units. In atomic units, $\alpha c=1, \mu_{0} / 4 \pi=\alpha^{2}$ and $\alpha^{-1}=137.036$. Here $c$ is the speed of light in a vacuum, $\mu_{0}$ is 
the permeability of free space, and $\alpha$ is the fine-structure constant. Using these factors Equation (2.7.6) becomes

$$
I=\frac{1}{8 \pi \alpha}\left(1+\varepsilon^{2}\right) F_{0}^{2} \quad \text { (Atomic units) }
$$

in atomic units. As most measurements are made in units of centimeters, seconds, volts, electron volts and so on, some common conversion factors are listed in Table 2.8.1

\begin{tabular}{ll}
\hline \multicolumn{1}{c}{ Atomic Unit } & \multicolumn{1}{c}{ Conversion } \\
\hline Field & $5.142 \times 10^{9} \mathrm{~V} / \mathrm{cm}$ \\
& \\
Irradiance & $6.436 \times 10^{15} \mathrm{~W} / \mathrm{cm}^{2}$ \\
Energy & $27.212 \mathrm{eV}$, or \\
& $4.360 \times 10^{-18 \mathrm{~J}}$ \\
& $1.602 \times 10^{-19} \mathrm{C}$ \\
Charge & $0.9109 \times 10^{-30} \mathrm{~kg}$ \\
Mass & $5.292 \times 10^{-11} \mathrm{~m}$ \\
Length & $4.134 \times 10^{16} \mathrm{~s}^{-1}$ \\
\hline
\end{tabular}

Table 2.8.1 Factors to convert atomic units to common experimental units.

The conversion of irradiance from MKS or CGS units to atomic units or from atomic units to MKS or CGS units is mishandled frequently. Appendix IV 
discusses the derivation of the atomic unit of irradiance and the conversion of the equations for irradiance from MKS and CGS units to obtain Equation (2.7.7). 


\section{Chapter 3-ANALXTIC YIELD SOLUTIONS FOR SEQUENTIAL IONIZATION}

\subsection{Introduction}

This chapter presents a digest of the procedure for obtaining analytic solutions to the sequential rate equations for several tunneling models that were given in reference [41]. General closed form solutions of the rate equations (2.5.1) are not possible because the higher indexed $p_{k}$ are multiple integrals of $p_{0}$. However,

good analytic approximations exist for several specific tunneling models for the spatio-temporal profile

$$
F_{0}(r, t)=\frac{\mathcal{F}_{0}}{\sqrt{1+\left(\frac{\lambda z}{\pi w_{0}^{2}}\right)^{2}}} \exp \left(-\frac{\frac{r^{2}}{w_{0}^{2}}}{1+\left(\frac{\lambda z}{\pi w_{0}^{2}}\right)^{2}}\right) \operatorname{sech}\left(1.76 \frac{t}{\tau}\right),
$$

that was mentioned previously in Equation (2.6.1). The analytic solutions provide insight into the dependences of the yields on the parameters of the system. Analytic expressions are given for the appearance irradiance at which a given number of ions should be produced. Expressions are also given for the threshold and saturation irradiances at which the probability of an atom being in a given charge state rises to 0.01 and falls to 0.01 , respectively. More importantly, the analytic solutions also show that the ion yields for sequential ionization increase in a characteristic way regardless of the charge state being considered. This characteristic nature of sequential ionization facilitates the identification of nonsequential features. 


\subsection{Analytic Solutions to the Rate Equations}

The formal solutions of the rate equations (Equations 2.5.1) at a fixed point in space are

$$
\left.\begin{array}{rl}
p_{0}(t) & =\exp \left(-\phi_{0}(t)\right) \\
p_{1}(t) & =\exp \left(-\phi_{1}(t)\right) \int_{-\infty}^{t} \exp \left(\phi_{1}(s)\right) \Gamma_{0}(s) p_{0}(s) d s \\
p_{2}(t) & =\exp \left(-\phi_{2}(t)\right) \int_{-\infty}^{t} \exp \left(\phi_{2}(s)\right) \Gamma_{1}(s) p_{1}(s) d s \\
& \ldots \\
p_{k}(t) & =\exp \left(-\phi_{k}(t)\right) \int_{-\infty}^{t} \exp \left(\phi_{k}(s)\right) \Gamma_{k-1}(s) p_{k-1}(s) d s \\
& \cdots \\
p_{n}(t) & =\int_{-\infty}^{t} \Gamma_{n-1}(s) p_{n-1}(s) d s
\end{array}\right\}
$$

where, as in Equations 2.5.1, $p_{k}$ is the probability that the atom is $k$ times ionized. The $\Gamma_{k}$ are the ionization rates for charge state $k$ and depend the applied electric field and the ionization potential. The $\Gamma_{k}$ are thus implicitly dependent on time and position. The quantity $\phi_{k}$ is the running integral of $\Gamma_{k}$

$$
\phi_{k}(t)=\int_{-\infty}^{t} \Gamma_{k}\left(t^{\prime}\right) d t^{\prime}
$$

For simple atom tunneling in argon by a hyperbolic-secant-squared pulse, Figure 3.2.1 shows the numerical integration of Equation (3.2.1) for the laser parameters of $I_{0}=3 \times 10^{16} \mathrm{~W} / \mathrm{cm}^{2}, \lambda=616 \mathrm{~nm}$ and $\tau=125 \mathrm{fs}$. The time evolution of the ionizing laser pulse, also shown in Figure 3.2.1, reconfirms the assertion[39] that the rising edge of the intense laser pulse creates the lower charge states. Simply put, the rapid tunneling ionization rates track the ultrashort pulse rise time. For 


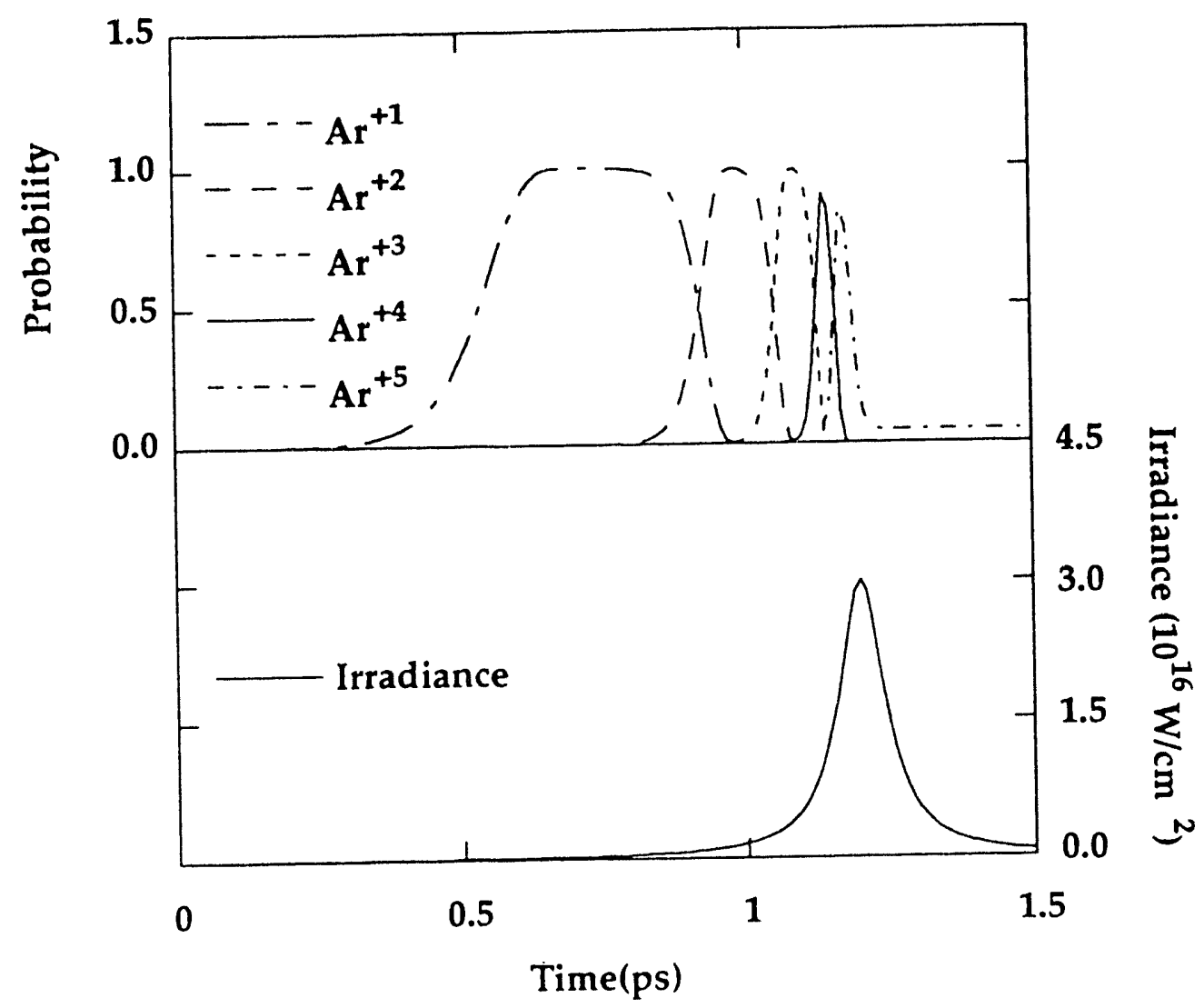

Figure 3.2.1 The plot of the numerical integration of Equation 3.2.1 and the temporal profile of the irradiance versus time shows that many of the ions appear early in the pulse. Few ions except for the most highly charged survive to the peak irradiance. The parameters of the hyperbolic-secant-squared pulse are $I_{0}=3 \times 10^{16} \mathrm{~W} / \mathrm{cm}^{2}$, $\lambda=616 \mathrm{~nm}$ and $\tau=125 \mathrm{f}$. The rates are simple tunneling rates.

any pulse with a peak irradiance higher than the threshold for a given rate, no ions of lower charge state will survive to see the higher irradiances. Also, for field ionization, ionization starts near the peak of the electric field for a given 
point on the temporal envelope of the pulse. Figure 3.2.1 illustrates the sequential nature of the equations graphically. This figure plotted on a linear vertical scale also emphasizes the importance of understanding 'prepulse' envelopes in experiments. The temporal separation of the higher indexed $p_{k}$ shows that the charge states exist sequentially in time for a fixed point in space. This allows simplification of Equation 3.2.1. The integrals in Equation 3.2.1 depend on all the probabilities of the lower charge states and are, in general, difficult to solve. However $p_{k}$ is accurately represented by its decaying edge $\exp \left(-\phi_{k}(t)\right)$. Applying this substitution to Equation 3.2.1 yields

$$
\left.\begin{array}{rl}
p_{0}(t) & =\exp \left(-\phi_{0}(t)\right) \\
p_{1}(t) & =\exp \left(-\phi_{1}(t)\right) \int_{-\infty}^{t} \exp \left(\phi_{1}(s)\right) \Gamma_{0}(s) \exp \left(-\phi_{0}(s)\right) d s \\
p_{2}(t) & =\exp \left(-\phi_{2}(t)\right) \int_{-\infty}^{t} \exp \left(\phi_{2}(s)\right) \Gamma_{1}(s) \exp \left(-\phi_{1}(s)\right) d s \\
& \ldots \\
p_{k}(t) & \approx \exp \left(-\phi_{k}(t)\right) \int_{-\infty}^{t} \exp \left(\phi_{k}(s)\right) \Gamma_{k-1}(s) \exp \left(-\phi_{k-1}(s)\right) d s \\
& \ldots \\
p_{n}(t) & \approx \int_{-\infty}^{t} \Gamma_{n-1}(s) \exp \left(-\phi_{n-1}(s)\right) d s .
\end{array}\right\}
$$

Equations (3.2.3) are exact solutions for $p_{0}$ and $p_{1}$ but approximate the other probabilities.

Equation (3.2.3) cannot be evaluated in closed form for an arbitrary laser pulse envelope. For very short laser pulses, treating the rates, $\Gamma_{k}$, as narrow square functions of time provides a very good representation of the final 
probabilities at the extinction of the laser pulse. The resulting integrations give[41]

$$
\begin{aligned}
p_{0}(\infty) & =\exp \left(-\phi_{0}\right) \\
p_{1}(\infty) & =\phi_{0} \frac{\exp \left(-\phi_{0}\right)-\exp \left(-\phi_{1}\right)}{\phi_{1}-\phi_{0}} \\
p_{2}(\infty) & =\phi_{1} \frac{\exp \left(-\phi_{1}\right)-\exp \left(-\phi_{2}\right)}{\phi_{2}-\phi_{1}} \\
\ldots & \\
p_{k}(\infty) & =\phi_{k-1} \frac{\exp \left(-\phi_{k-1}\right)-\exp \left(-\phi_{k}\right)}{\phi_{k}-\phi_{k-1}} \\
& \ldots \\
p_{n}(\infty) & =1-\exp \left(-\phi_{n-1}\right) .
\end{aligned}
$$

Here

$$
\phi_{k} \equiv \phi_{k}(\infty)
$$

is the total area under the rate curve. The value of each $p_{k}$ in Equation (3.2.4) is between zero and one. Thus the $p_{k}$ given by the equation can be still interpreted as the probability for the charge state $k$ to be present for a given peak laser irradiance. Figures 3.2.2 and 3.2.3 show the probabilities for simple tunneling in argon versus irradiance obtained from direct numerical integration of the rate equations and Equation (3.2.4), respectively. The parameters of the circularly polarized laser pulse are those used for Figure 3.2.1. The plots show no discernible difference between the exact solution and the results of Equation (3.2.4) thus validating the simplifying assumptions. 


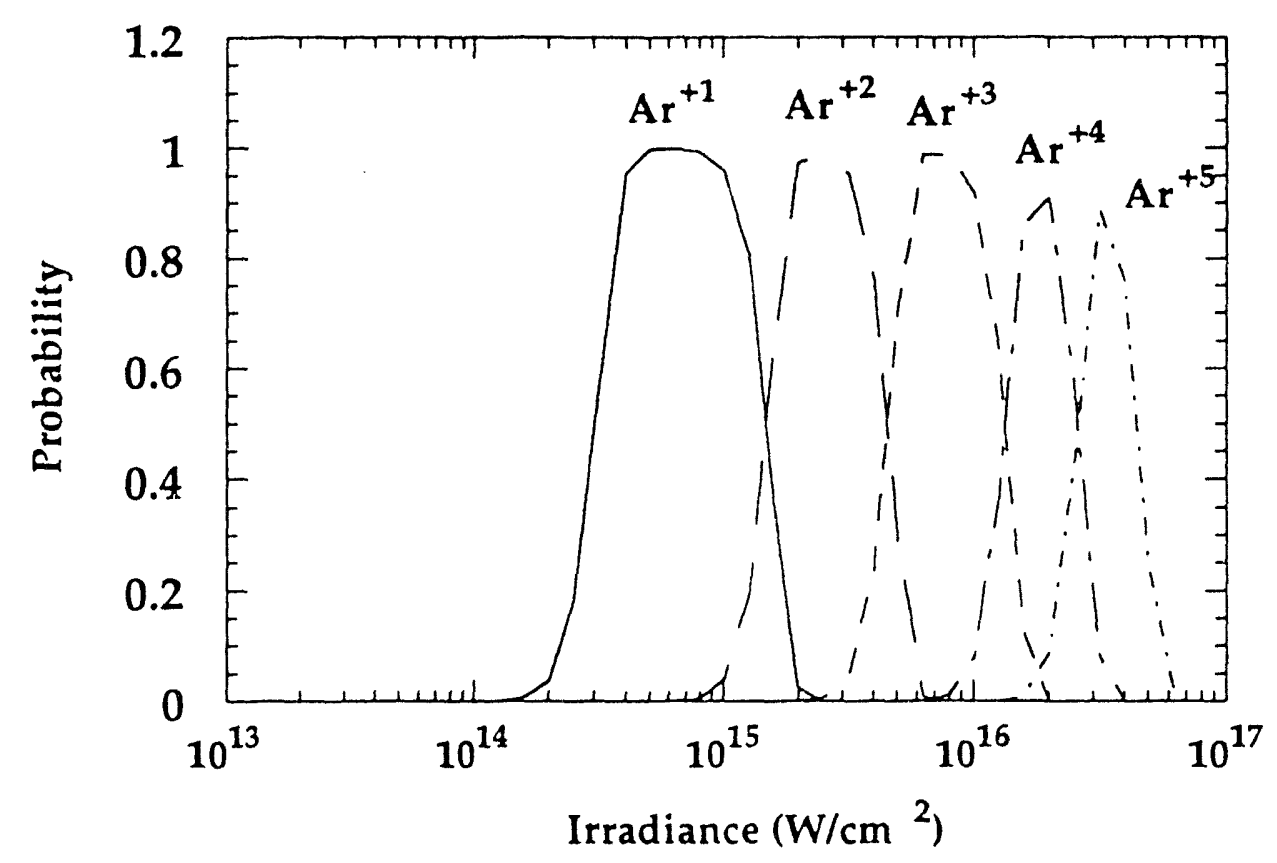

Figure 3.2.2 The numerically calculated ion balance of the charge states of argon. The probabilities of charge states $\mathrm{Ar}^{+1}, \mathrm{Ar}^{+2}, \mathrm{Ar}^{+3}, \mathrm{Ar}^{+4}$, and $\mathrm{Ar}^{+5}$ [final probability of Equation (3.2.3)] are plotted as a function of irradiance for the simple tunneling model for circular polarization. 


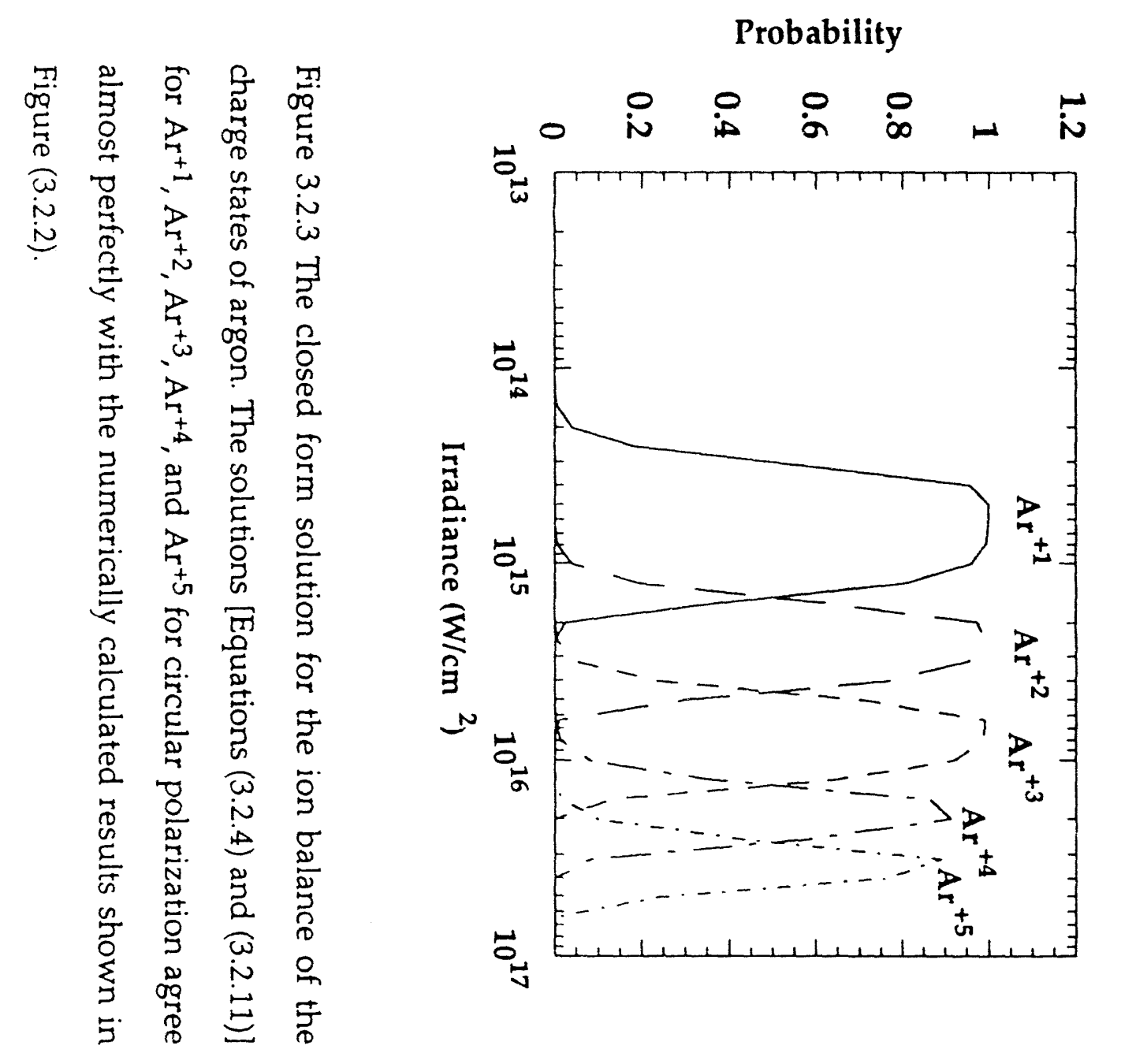


Now we will consider the analytic solutions for the simple atom model. As noted in Chapter 2, for simple static tunneling in a hydrogenic atom with ionization potential, $E$, in an applied field $F$ given by (2.7.1) (both in atomic units), the ionization rate is

$$
\Gamma=6 \omega_{0}(2 E)\left(\frac{\frac{2}{3}(2 E)^{3 / 2}}{F}\right) \exp \left(-\frac{\frac{2}{3}(2 E)^{3 / 2}}{F}\right),
$$

This rate applies to the instantaneous electric field. For a linearly polarized field, the rate must be averaged over a cycle of the wave. For a circularly polarized field, however, the field has a constant magnitude during a cycle of the wave and the cycle-averaged rate is the rate for the spatially dependent field magnitude, $F_{0}$,

$$
\Gamma=6 \omega_{0}(2 E)\left(\frac{\frac{2}{3}(2 E)^{3 / 2}}{F_{0}}\right) \exp \left(-\frac{\frac{2}{3}(2 E)^{3 / 2}}{F_{0}}\right)
$$

The linear and circularly polarized rates are the extremes of the rates with respect to ellipticity. Consider the case of circular polarization. For charge state $k$

$$
\phi_{k}=\left(6 \omega_{0} E_{k}\right) \int_{-\infty}^{\infty} d t \frac{\frac{2}{3} E_{k}^{3 / 2}}{F_{0}} \exp \left(-\frac{\frac{2}{3} E_{k}^{3 / 2}}{F_{0}}\right) .
$$

Here $E_{k}$ is twice the ionization potential of charge state $k$ in atomic units. Performing the integration gives

$$
\phi_{k}=\left(6 \omega_{0} E_{k}\right)\left(\frac{2 \tau}{1.76}\right) \frac{\frac{2}{3} E_{k}^{3 / 2}}{F_{0}} K_{1}\left(\frac{\frac{2}{3} E_{k}^{3 / 2}}{F_{0}}\right) .
$$


where $K_{1}$ is the modified Bessel function of order one[42].

Now consider the scaling that arises by introducing the scaled electric field,

$$
F_{k 0}=\frac{F_{0}}{\frac{2}{3} E_{k}^{3 / 2}} .
$$

The total area under the rate curve becomes

$$
\phi_{k}=\left(6 \omega_{0} E_{k}\right)\left(\frac{2 \tau}{176}\right) \frac{1}{F_{k 0}} K_{1}\left(\frac{1}{F_{k 0}}\right) .
$$

For a given point in space, Equation (3.2.4) gives the final ion balance as a function of irradiance. It describes how the population of charge state $k$ increases and diminishes with irradiance. The irradiances marking the onset and extinction of $p_{1}$ bear heavily on the shape of the ion yield curve for the singly ionized atom because the yield is obtained by integrating $p_{1}$ of Equation (3.2.4) over all space. Let us call the irradiance corresponding to the onset of $p_{1}$ the threshold irradiance, and the irradiance corresponding to the extinction of $p_{1}$ the saturation irradiance. For both saturation and threshold the defining probability of the atom being in the given charge state taken to be $1 \%$. This definition of the saturation irradiance differs from that of reference [12] where saturation is defined in terms of the multiphoton ionization description. 


\subsection{Threshold and Saturation Irradiances}

To determine the threshold and saturation irradiances of the probabilities in Equation (3.2.4), we need to know the behavior of the $\phi_{k}{ }^{\prime} s$ with respect to the field strength. Figure 3.3.1 shows that $\phi_{0}$, for example, increases monotonically with increasing field strength. From the asymptotic limit of the Bessel function in Equation (3.2.7), both $\phi_{k}$ and $\phi_{k+1}$ are of order $\exp \left(-2 E_{k}^{3 / 2} / 3 F_{0}\right)$ for field strengths that are small compared to the binding fields. Therefore $p_{k+1}$ is approximately equal to $\phi_{k}$ because the ratio $\left[\exp \left(-\phi_{k}\right)-\exp \left(\phi_{k+1}\right)\right] /\left[\phi_{k+1}-\phi_{k}\right]$ is close to one:

$$
p_{k+1}=\phi_{k}\left(1+\frac{1}{2}\left(\phi_{k}+\phi_{k+1}\right)+\cdots\right) \geq \phi_{k}
$$

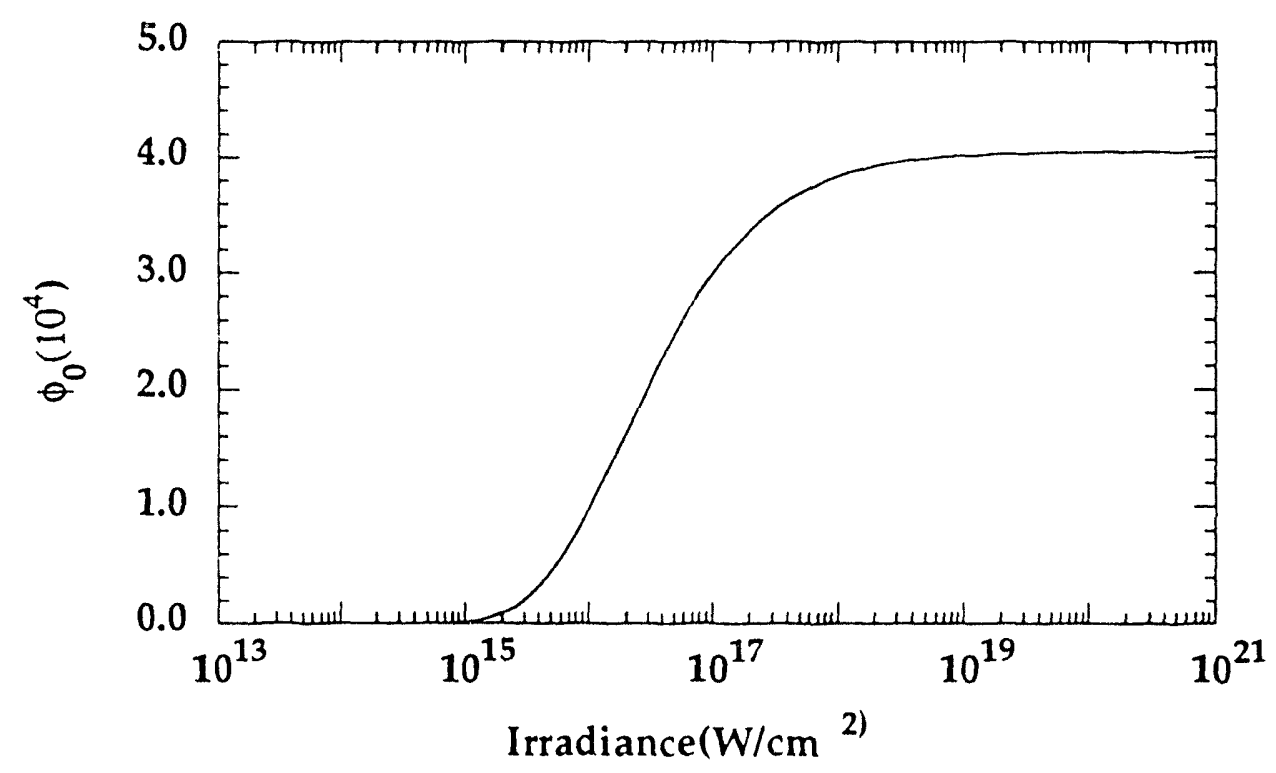

Figure 3.3.1 A plot of the total area under the rate curve as a function of irradiance. $\phi_{k}$, which can be thought of as the degree of ionization of charge state $k$, increases monotonically with increasing irradiance. The plot is for simple-atom model ionization of Ar for circular polarization. 
So for the simple atom model the final probability is

$$
p_{k+1}=\left(6 \omega_{0} E_{k}\right)\left(\frac{2 \tau}{1.76}\right)^{\frac{2}{3} E_{k}^{3 / 2}} \sqrt{\frac{\pi}{2} \frac{3 F}{2 E_{k}^{3 / 2}}} \exp \left(-\frac{\frac{2}{3} E_{k}^{3 / 2}}{F_{0}}\right)
$$

where the asymptotic limit of $K_{1}$ is substituted in Equation (3.2.9). The exponential in Equation (3.3.2) gives the rising edge of $p_{k+1}$ and shows that $p_{k+1}$ rises steeply for small F. Although $p_{k+1}$, according to Equation (3.3.2), is zero only for $F_{0}$ equal to zero, it is however vanishingly small for small $F_{0}$. We define the threshold field strength as the field for which $p_{k+1}$ is equal to $p_{t h r}$. Solving Equation (3.3.2) for the threshold field strength gives

$$
F_{k+1}^{\text {thrs }}=\frac{\frac{2}{3} E_{k}^{3 / 2}}{\ln \left(\left(6 \omega_{0} E_{k}\right)\left(\frac{2 \tau}{1.76}\right) \sqrt{\frac{\pi}{2}} / p_{t h r}\right)}
$$

The threshold field strength is relatively insensitive to $p_{\text {thr }}$ (by a factor of a few) because of the logarittum function in Equation (3.3.3). The defining value of $p_{t h r}$ is also outweighed by the numerator of the logarithm function, which is of order of $10^{4}$. This is to be expected for any high order process. Using Equation (2.7.7) for $\varepsilon=1$, the simple atom threshold irradiance is given by

$$
I_{k+1}^{\text {thres }}=\frac{1}{9 \pi \alpha} E_{k}^{3} /\left[\ln \left(\left(6 \omega_{0} E_{k}\right)\left(\frac{2 \tau}{1.76}\right) \sqrt{\frac{\pi}{2}} / p_{t h r}\right)\right]^{2} .
$$

Again $\alpha$ is the fine-structure constant. Table 3.3.1 lists the threshold irradiances derived in Equation (3.3.4) for $k=0, \ldots, 4$ and $p_{t h r}=p_{\text {sat }}=0.01$. I use these values for $p_{\text {sat }}$ and $p_{t h r}$ in all discussions of the validity of equations for threshold or saturation. A comparison of the threshold irradiances in Table 3.3.1 and Figure 3.2.2 demonstrates the accuracy of Equation (3.3.4). Note also the diminishing 


\begin{tabular}{cccc}
\hline \hline & & & \\
Charge State & $I^{\text {thresh }}\left(\mathrm{W} / \mathrm{cm}^{2}\right)$ & $I^{\text {sat }}\left(\mathrm{W} / \mathrm{cm}^{2}\right)$ & $I^{\text {sat }} / I^{\text {thresh }}$ \\
\hline 1 & $2.03(14)$ & $2.68(15)$ & 13.2 \\
+2 & $1.02(15)$ & $7.94(15)$ & 7.8 \\
+3 & $3.11(15)$ & $2.34(16)$ & 7.5 \\
+4 & $9.40(15)$ & $4.42(16)$ & 4.7 \\
+5 & $1.81(16)$ & $7.61(16)$ & 4.2 \\
\hline \hline
\end{tabular}

Table 3.3.1 Argon threshold and saturation irradiances for the simple atom model for circular polarization calculated using Equations (3.3.4) and (3.3.7). $\mathrm{p}_{\mathrm{thr}}=.01=\mathrm{p}_{\text {sat }}$. The parameters used are $\omega_{0}=4.1 \times 10^{16} \mathrm{sec}^{-1}, \tau=1.25 \times 10^{-13} \mathrm{sec}$, and the $E_{k}$ 's are twice the ionization potentials of argon in atomic units. 
ratio $I^{\text {sat }} / I^{\text {thrsh }}$ for increasing charge state or ionization potential. This addresses the steepness of the characteristic sequential curve.

The threshold irradiance defined in the above manner differs from the experimental appearance irradiance, $I_{k+1}^{a p p}$. While a given ionization rate defines the threshold irradiance at a given point in space, the detection of a few ions provides the practical definition of the appearance irradiance. Since the number of ions detected depends on the ambient gas density, the detection efficiency and the size and shape of the focal volume, the appearance irradiance depends on these factors as well. The threshold irradiance, which is defined only in terms of the atomic properties of the system, is completely independent of these factors. For this reason the appearance field and appearance irradiance are determined in Section 3.5 in conjunction with the ion yield calculations.

Define the saturation field strength of $p_{k+1}$ as the field strength where $p_{k+1}$ decreases to the value of $p_{s a t} \leq .01$. As shown in Figure 3.2.2, the saturation irradiance is moderately greater than the threshold irradiance. In this irradiance regime, $\phi_{k}$ and $\phi_{k+1}$ are both large. However $\phi_{k}$ is larger than $\phi_{k+1}$, so $\exp \left(-\phi_{k}\right)$ is negligible compared to $\exp \left(-\phi_{k+1}\right)$. Then, for high field strengths, $p_{k}$ is approximately equal to $\exp \left(-\phi_{k}\right)$ because

$$
\begin{aligned}
p_{k+1} & =\frac{1}{\frac{\phi_{k+1}}{\phi_{k}}-1}\left[\exp \left(-\phi_{k}\right)-\exp \left(-\phi_{k+1}\right)\right] \\
& =\frac{1}{0-1}\left[-\exp \left(-\phi_{k+1}\right)\right] .
\end{aligned}
$$

In this irradiance regime, it is also valid to use the asymptotic form of $\phi_{k+1}$ therefore, the saturation field strength is 


$$
F_{k}^{\text {sat }}=\frac{\frac{2}{3} E_{k}^{3 / 2}}{\ln \left(\left(6 \omega_{0} E_{k}\right)\left(\frac{2 \tau}{1.76}\right) \sqrt{\frac{\pi}{2}} / 4.605\right)}
$$

The value $4.605=-\ln (0.01)$ in Equation (3.3.6) arises from setting $p_{k+1}=p_{\text {sat }}=0.01$ in Equation (3.3.5). Using Equation (2.7.7) for $\varepsilon=1$, the corresponding saturation irradiance is

$$
I_{k}^{\text {sat }}=\frac{1}{9 \pi \alpha} E_{k}^{3} /\left[\ln \left(\left(6 \omega_{0} E_{k}\right)\left(\frac{2 \tau}{176}\right) \sqrt{\frac{\pi}{2}} / 4.605\right)\right]^{2}
$$

Inspection of Table 3.3.1 and Figure 3.2.2 shows that Equation (3.3.7) does not predict the saturation irradiance as accurately as Equation 3.3.4 predicts the threshold irradiance. The inaccuracy occurs because both $\phi_{k}$ and $\phi_{k+1}$ must be very large for the approximation of Equation (3.3.5) to be valid. $\phi_{k}$ and $\phi_{k+1}$ are, however, only of the order of a few. Like the threshold irradiance, the saturation irradiance is independent of the gas density and focal parameters because it is defined in terms of probabilities and not in terms of integrated ion count.

Equations (3.3.1) and (3.3.5) give a physical interpretation of the probability distribution function $p_{k+1}$. The rising edge of $p_{k+1}$ is $\phi_{k}$, and its decaying edge is $\exp \left(-\phi_{k+1}\right)$. Furthermore, since $\phi_{k}$ is the total area under the $\Gamma_{k}$ rate curve, $\phi_{k}$ represents the degree of ionization of charge state $k$. Until the laser irradiance rises enough to ionize charge state $k+1$, the probability of ions being in charge state $k+1$ increases with increasing laser irradiance. However when the laser irradiance increases even further, the laser pulse can also ionize charge state $k+1$, so the probability of the ions being in charge state $k$ decays like $\exp \left(-\phi_{k+1}\right)$. 


\subsection{Ion Yield Curves}

Determining the ion yield requires integrating the ionization probabilities over all space; integrating over iso-irradiance shells greatly simplifies the problem. In mathematical terms, a change of integration variable from volume to irradiance simplifies the volume integral. We can enhance our physical understanding of how the change of variable simplifies the spatial integration and of the spatial inhomogeneity of the ionization in the focal region of the laser, by plotting the spatial distributions for $\mathrm{Ar}^{+2}$ and $\mathrm{Ar}^{+4}$ produced in a Gaussian beam with peak irradiance of $10^{17} \mathrm{~W} / \mathrm{cm}^{2}$ in Figure 3.4.1. The ions fall within

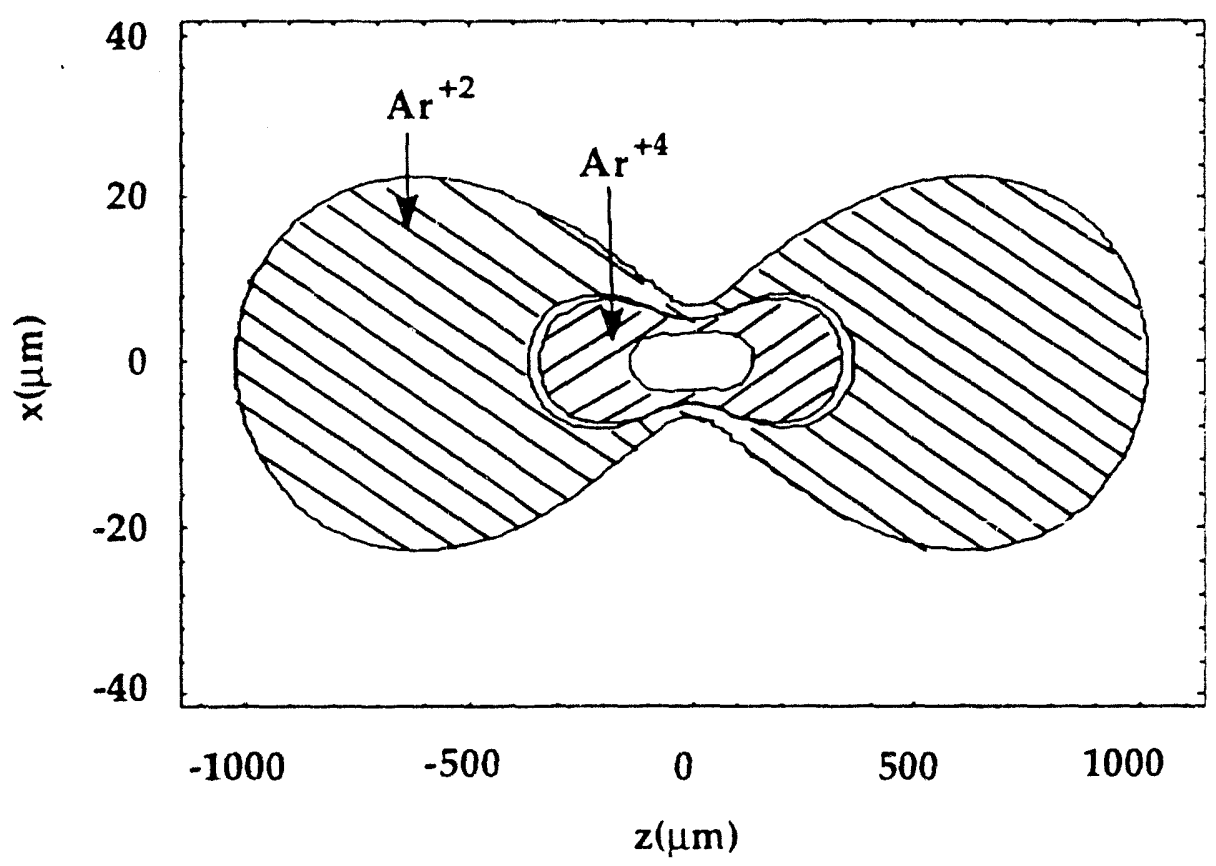

Figure 3.4.1 The spatial distributions of ions produced in a Gaussian beam. The distributions are for $\mathrm{Ar}^{+2}$ and $\mathrm{Ar}^{+4}$ with peak irradiance of $10^{17} \mathrm{~W} / \mathrm{cm}^{2}$. The focus has an $\mathrm{f}$ number of 5 . The ions fall within borders defined by the threshold and saturation isoirradiance contours. 
borders defined by the threshold and saturation iso-irradiance contours. The higher charge states are close to the focus where the laser irradiance is higher, and the lower charge states are further from the focus where the laser irradiance is lower. After passage of the pulse, the small temporal overlap of the $p_{k}{ }^{\prime} s$ for a fixed irradiance appears as low spatial overlap of nonconsecutive charge states such as $\mathrm{Ar}^{+2}$ and $\mathrm{Ar}^{+4}$.

For a Gaussian beam of peak irradiance $I_{0}$, the volume inside an isoirradiance boundary defined by $I$, is[12]

$$
V(I)=V_{0}\left[\frac{2}{9} \xi^{3}+\frac{4}{3} \xi-\frac{4}{3} \operatorname{atan}(\xi)\right]
$$

where

$$
\xi=\sqrt{\frac{I_{0}}{I}-1} \text {, and } V_{0}=\frac{\pi^{2} w_{0}^{4}}{\lambda}
$$

Equation 3.4.2 is the change of variable needed for the spatial integral. Let $N_{k+1}$ be the number of ions of charge $k+1$ at the extinction of the laser pulse. This ion yield $N_{k+1}$ for ion $k+1$ as a function of the peak irradiance is

$$
N_{k+1}=\rho \int_{0}^{l_{0}} \frac{d V}{d I} p_{k+1} d I
$$

where $\rho$ is the density of the target gas. 


\subsection{Low Irradiance Limit of the Ion Yield and Appearance Irradiance}

For the simple-atom model with a circularly polarized field, the ion-yield curve for laser irradiances below the theoretical threshold irradiance (defined for $\left.p_{\text {thr }} \leq .01\right)$ can be obtained in closed form by substituting the low irradiance form of $p_{k+1}$, Equation (3.3.2), into Equation (3.4.3) and performing the resulting integration[43].

$$
N_{k+1}=\left(6 \omega_{0} E_{k}\right)\left(\frac{2 \tau}{176}\right) \rho\left(\pi V_{0}\right)\left(\mathcal{F}_{k 0}+\mathcal{F}_{k 0}{ }^{2}+\mathcal{F}_{k 0}{ }^{3}\right) \exp \left(-\frac{1}{\mathcal{F}_{k 0}}\right),
$$

where the peak scaled field, $\mathcal{F}_{k 0}$, is

$$
\mathcal{F}_{\text {ko }}=\frac{\mathcal{F}_{0}}{\frac{2}{3} E_{k}^{3 / 2}} .
$$

Note that $\mathcal{F}_{k 0}$ is used here to clarify the notation of the original work where $F_{k}$ signified both the scaled field of Equation (3.2.10) and the peak scaled field of Equation (3.5.2). Figure 3.5.1 compares Equation (3.5.1) with the numerically integrated results. The two curves are numerically identical for irradiances below the theoretical threshold irradiance and diverge for irradiances above it.

The experimental appearance irradiance can be derived from Equation (3.5.1) by finding the irradiance at which $N_{k+1}$ is equal to a few, $N_{a p}$. For example the appearance field strength for $N_{a p}$ ions is

$$
\mathcal{F}_{k+1}^{\text {appear }}=\frac{\frac{2}{3} E_{k}^{3 / 2}}{\ln \left(\left(6 \omega_{0} E_{k}\right)\left(\frac{2 \tau}{1.76}\right) \rho\left(\pi V_{0}\right) / N_{a p}\right)} .
$$

Using Equation (2.7.7) for $\varepsilon=1$, the corresponding appearance irradiance is then 


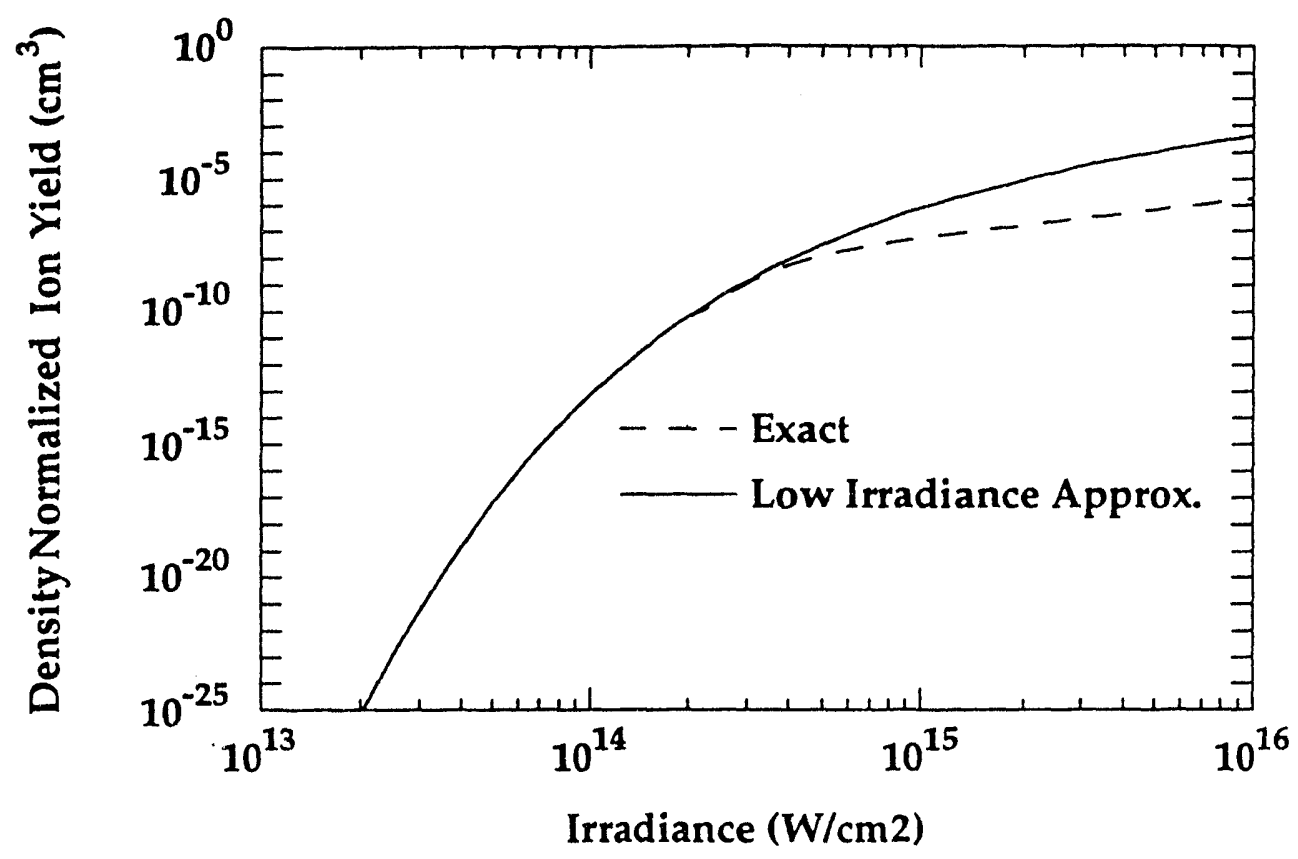

Figure 3.5.1 The low irradiance limit of the density normalized ion yield is compared to the numerically calculated results. $N_{1} / \rho$ (Equation 3.5.1) is given for $\mathrm{Ar}^{+1}$. The two curves diverge at the theoretical threshold irradiance of Equation (3.3.3), whose value in Table 3.3.1 is $2.03 \times 10^{14} \mathrm{~W} / \mathrm{cm}^{2}$.

$$
I_{k+1}^{\text {appear }}=\frac{1}{9 \pi \alpha} E_{k}^{3} /\left[\ln \left(\left(6 \omega_{0} E_{k}\right)\left(\frac{2 \tau}{1.76}\right) \rho\left(\pi V_{0}\right) / N_{a p}\right)\right]^{2}
$$

Here $I_{k+1}^{a p p e a r}$ is the peak irradiance of the envelope of the pulse. With $N_{a p}$ set equal to one, Figure 3.5.2 shows Equation (3.5.4) plotted as a function of the ionization potential for three gas densities. The plot shows the weak density dependence of the appearance irradiance. The appearance irradiance is insensitive to all the parameters that are in the logarithmic denominator of Equation (3.5.4). Because 


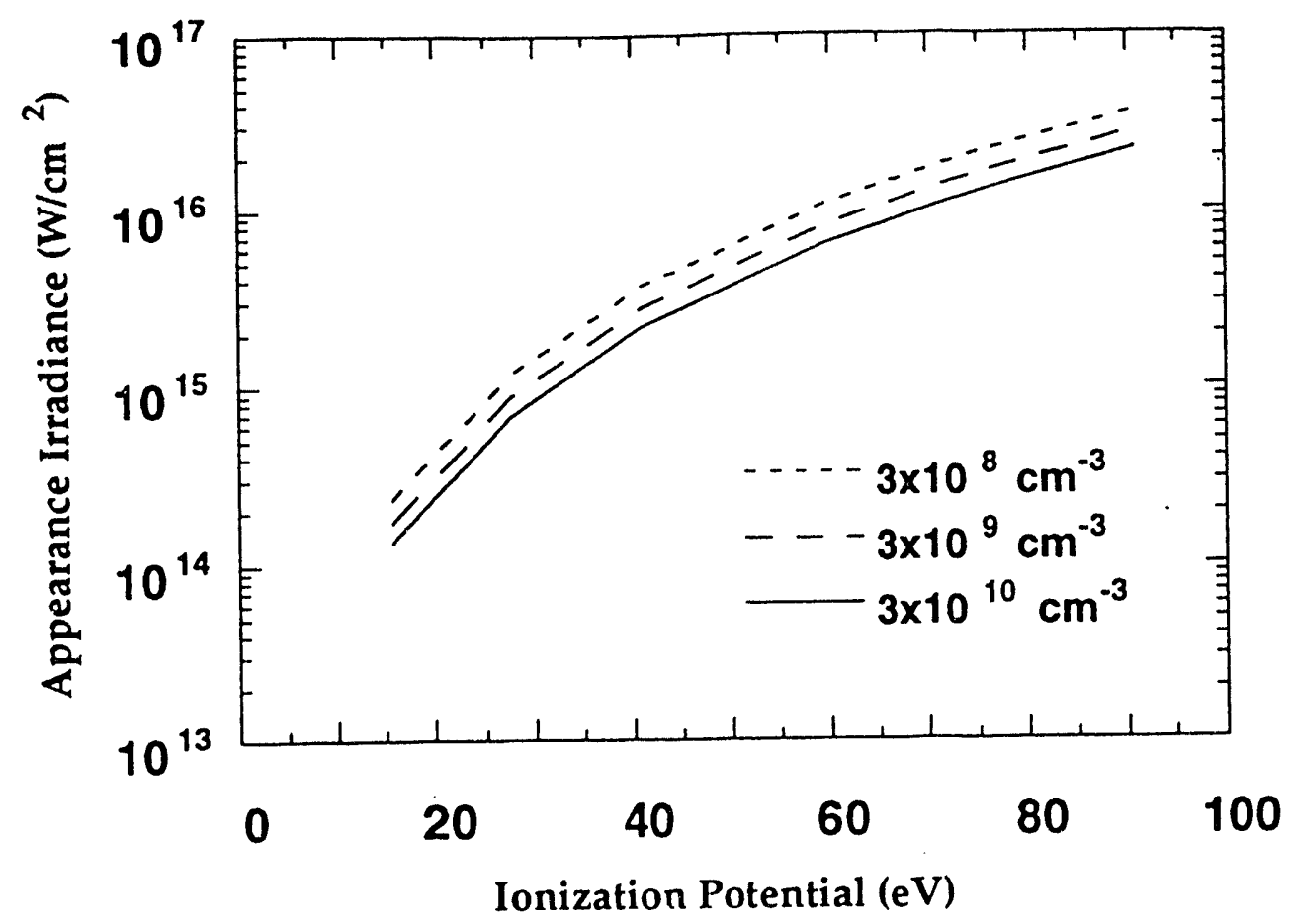

Figure 3.5.2 The simple atom model appearance irradiance for $\mathrm{Ar}^{+1}$ as a function of irradiance at three densities for circular polarization. The three target gas densities are $3 \times 10^{8}, 3 \times 10^{9}$ and $3 \times 10^{10} \mathrm{~cm}^{-3}$.

of this insensitivity the appearance yield is good measure of irradiance. On the other hand, this insensitivity hinders our ability to distinguish atomic ionization models based on appearance irradiances.

The important physics in Equation (3.5.4) is that the appearance irradiance obeys a predominantly cubic power law with respect to the ionization potential and departs from this power law by the weak logarithmic denominator. The logarithmic term decreases the growth rate of the appearance irradiance as the ionization potential increases. Furthermore the cubic power law is common to the simple tunneling, ADK and MPI models, while only the simple tunneling and 
ADK models possess the square of $\ln \left(E_{k}\right)$ factor in their denominators. On the other hand, the MPI model has in its denominator the factor of $E_{k}^{1 / n}$, where $\mathrm{n}$ is the number of photons needed for ionization, which is an upper bound-see Equation (4.1.37) in [42] of $\ln \left(E_{k}\right)$. The cubic power law arises from the $(2 / 3) E_{k}^{3 / 2}$ term for normalizing the laser field strength in the rate laws. This term represents the intra-atomic forces acting on the electron to be ionized by the laser, and thus is the scale by which the laser field is measured[27] On the other hand, the $E_{k}$ in the $\ln \left(E_{k}\right)$ term of Equation (3.5.3) arises from the numerical frequency factor $\left(6 \omega_{0} E_{k}\right)$ in the front of the rate law. This factor is the frequency of the electron's motion in the atom, and is also the scale by which the ionization rate is measured[27, 44]. Therefore the intra-atomic force acting on the electron controls the numerator of the appearance irradiance and the frequency of the electron's motion controls the denominator.

\subsection{High Irradiance Limit of the Ion Yield and Saturation Irradiance}

The ion yield for laser irradiances above the saturation irradiance can be obtained in closed form by substituting the asymptotic form of the volume Jacobian,

$$
\frac{d V}{d I} \approx \frac{V_{0}}{3} \frac{I_{0}^{3 / 2}}{I^{5 / 2}}
$$

in Equation (3.4.3). The integration domain in Equation (3.4.3) is effectively $I_{k+1}^{\text {thr }}\left(p_{t h r}=.01\right)$ to $I_{k+1}^{\text {sat }}\left(p_{\text {sat }}=.01\right)$ because $p_{k+1}$ is vanishingly small for irradiances beyond this range (see Figure 3.2.2). Within this range $p_{k+1}$ can be approximated by the unit function giving the ion yield as:

$$
N_{k+1}=\frac{2}{3} \rho\left(\frac{2}{9} V_{0}\right)\left(\frac{1}{\left(I_{k+1}^{\text {thr }}\right)^{3 / 2}}-\frac{1}{\left(I_{k+1}^{\text {sat }}\right)^{3 / 2}}\right) I_{0}^{3 / 2}
$$


The $2 / 3$ factor in Equation (3.6.2) arises as follows. If we had approximated $p_{k+1}$ by a parabola instead of the rectangular function, we would have obtained a more accurate approximation than Equation (3.6.2). The numerical factor is approximately equal to $2 / 3$, the ratio of the area of a parabola inscribed by a rectangular function to the area of the rectangular function.

Figure (3.6.1) compares Equation (3.6.2) with the numerically integrated results for $\mathrm{Ar}^{+1}$. We find that the numerically integrated result approaches its

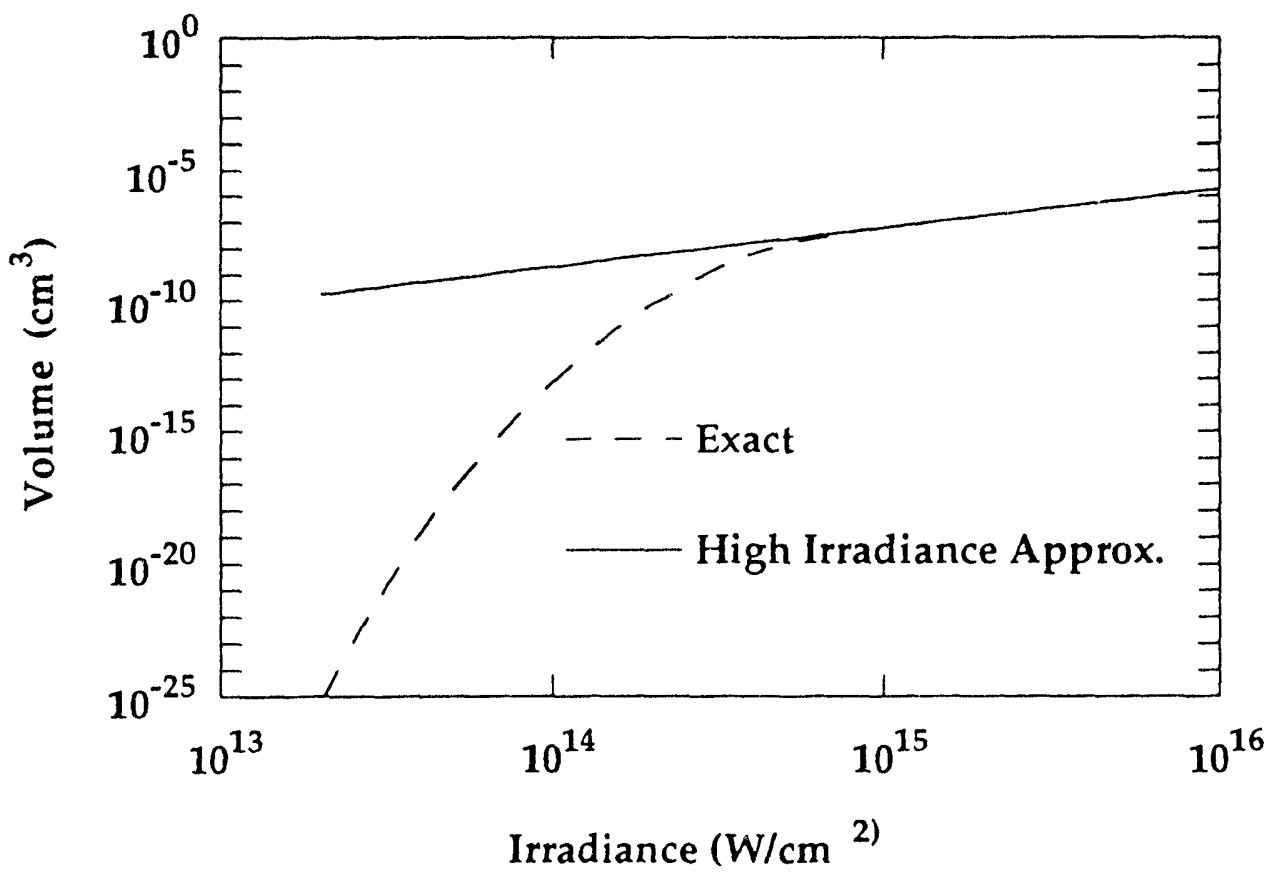

Figure 3.6.1 The high irradiance limit of the density normalized ion yield is compared to the numerically calculated results. $N_{1} / \rho$ (Equation 3.6.2) is given for $\mathrm{Ar}^{+1}$. The two curves converge at the saturation irradiance of Equation (3.3.7), whose value in Table 3.3.1 is $2.68 \times 10^{15} \mathrm{~W} / \mathrm{cm}^{2}$. 
asymptote, Equation (3.6.2), at the saturation irradiance given by Equation (3.3.7) (the value from Table 3.3 .1 is $2.68 \times 10^{15} \mathrm{~W} / \mathrm{cm}^{2}$ ). The ion yield curve converges to its asymptotic limit at the saturation irradiance where the integral of Equation (3.4.3) slows down to a $3 / 2$ power rate of growth with the peak irradiance. When $I_{0}$ increases from 0 to $I^{\text {sat }}$, the integral in Equation (3.4.3) increases for two reasons: 1) the range of integration increases, and 2) the integrand is an increasing function of $I_{0}$. However as $I_{0}$ goes beyond $I^{\text {sat }}, p_{k+1}$ truncates the integrand. The truncation is effected by setting the upper limit of integration equal to $I^{\text {set }}$ The truncation cuts off an avenue of growth for the integral, and thus reduces the integral's growth rate to a $3 / 2$ power law. This asymptotic dependence is commonly known as the "volume expansion effect[12]".

\subsection{Integrating the Other Rate Laws}

I will demonstrate the integration method for the case of a complex atom in a circularly polarized field. The complex atom or ADK rate, Equation (2.3.11), can be written as

$$
\Gamma_{c a}=\gamma_{c a}\left(\frac{1}{F_{k 0}}\right)^{P+1} \exp \left(-\frac{1}{F_{k 0}}\right)
$$

where $P=2 n-|m|-2$, and $F_{k 0}$ is the same normalized field strength given in Equation (3.2.10). The factor $\gamma_{c a}$ is

$$
\gamma_{c a}=\omega_{0} \tilde{C}_{n l}^{2} G_{l m} \frac{E_{k}}{2} .
$$

Here

$$
\tilde{C}_{n l}^{2}=\frac{2^{2 n}}{n \Gamma(n+l+1) \Gamma(n-l)} 3^{2 n-1}
$$


and

$$
G_{l m}=\frac{(2 l+1)(l+|m|) !}{6^{|m|}(|m|) !(l-|m|) !}
$$

Note that reference [41] used a form for $\tilde{C}_{n l}^{2}$ that was based on the form for $C_{n \eta 1}^{2}$. given in reference [31] for $n^{*}>l$. The form above is based on the form for $C_{n}^{2} \%$ for arbitrary $l$ given in Equation 19 of reference [31]. Let us cast Equation (3.7.1) into the form of the simple atom rate law by rewriting it as

$$
\Gamma_{c a}=\lim _{\beta \rightarrow 1} \gamma_{c a}\left(-\frac{\partial}{\partial \beta}\right)^{p} \frac{1}{F_{k 0}} \exp \left(-\frac{\beta}{F_{k 0}}\right)
$$

Here $\beta$ is a dummy variable that is set equal to one at the end of the calculation.

The total time integral of Equation (3.7.5) can be written as

$$
\phi_{c a}=\lim _{\beta \rightarrow 1} \gamma_{c a} \int_{-\infty}^{\infty} d t\left(-\frac{\partial}{\partial \beta}\right)^{P} \frac{1}{F_{k 0}} \exp \left(-\frac{\beta}{F_{k 0}}\right)
$$

Interchanging the order of integration and differentiation gives

$$
\phi_{c a}=\lim _{\beta \rightarrow 1} \gamma_{c a}\left(-\frac{\partial}{\partial \beta}\right)^{p} \int_{-\infty}^{\infty} d t \frac{1}{F_{k 0}} \exp \left(-\frac{\beta}{F_{k 0}}\right) .
$$

Furthermore Equation (3.7.7) can also be expressed as

$$
\phi_{c a}=\lim _{\beta \rightarrow 1} \gamma_{c a}\left(-\frac{\partial}{\partial \beta}\right)^{p} \frac{1}{\beta} \int_{-\infty}^{\infty} d t \frac{\beta}{F_{k 0}} \exp \left(-\frac{\beta}{F_{k 0}}\right)
$$

Performing the integration, Equation (3.7.8) can be expressed in closed form as

$$
\phi_{c a}=\lim _{\beta \rightarrow 1} \gamma_{c a}\left(\frac{2 \tau}{1.76}\right)\left(-\frac{\partial}{\partial \beta}\right)^{P} \frac{1}{\beta}\left[\frac{\beta}{F_{k 0}} K_{1}\left(\frac{\beta}{F_{k 0}}\right)\right]
$$

where $K_{1}$ is the Modified Bessel Function of the Second Kind of order 1. 
Note that the prescription of setting $n=n$ * does not work in Equation (3.7.9) because the exponent $P$ must be an integer. The effective quantum numbers may be used by making the further approximation

$$
\phi_{c a}=\lim _{\beta \rightarrow 1}\left(\omega_{0} \tilde{C}_{n * *}^{2} G_{l m} \frac{E_{k}}{2}\right)\left(\frac{2 \tau}{1.76}\right)\left(-\frac{\partial}{\partial \beta}\right)^{\beta} \frac{1}{\beta}\left[\frac{\beta}{F_{k 0}} K_{1}\left(\frac{\beta}{F_{k 0}}\right)\right]
$$

where $\tilde{P}$ is the nearest integer to $2 n^{*}-|m|-2$. This method can also be used to find the low irradiance form of the ion yield curve, which is the volume integral of Equation (3.7.9). Furthermore cycle-averaging is the process of integrating over the phase of the $\mathrm{E}$ field for a linearly polarized field. Since differentiation by $\beta$ commutes with both volume integration and phase integration, the other formulae in Tables 3.7.1 to 3.7.5 can be derived explicitly by this integration method. For the convenience of the reader The ADK threshold and saturation irradiances for linear and circular polarization are given in Table 3.7.6. The irradiances are based on the equations of Tables 3.7.3 and 3.74 using the effective principle quantum numbers of the ground state configurations. The $m$ quantum numbers are set to 0 . The saturation values for $\mathrm{He}^{+2}$ are not given because the saturation irradiance is defined as the point at which the final probability of being in the charge state $k$ has dropped to 0.01 due to ionization to charge state $k+1$. Since helium only has two electrons, the saturation irradiance-as defined here-does not exist for $\mathrm{He}^{+2}$. 


$$
\begin{array}{ll}
\Gamma & \gamma \frac{1}{F_{k 0}} \exp \left(-\frac{1}{F_{k 0}}\right) \\
\gamma & 6 \omega_{0} E_{k} \\
\phi & \gamma\left(\frac{2 \tau}{176}\right)\left(\frac{1}{F_{k 0}}\right) K_{1}\left(\frac{1}{F_{k 0}}\right) \\
N_{k+1} & \gamma\left(\frac{2 \tau}{1.76}\right)\left(\pi \rho V_{0}\right)\left(\mathcal{F}_{k 0}+\mathcal{F}_{k 0}^{2}+\mathcal{F}_{k 0}{ }^{3}\right) \exp \left(-\frac{1}{\mathcal{F}_{k 0}}\right) \\
F_{k+1}^{\text {thres }} & \frac{2}{3} E_{k}^{3 / 2} / \ln \left[\gamma\left(\frac{2 \tau}{1.76}\right) \sqrt{\frac{\pi}{2}} / .01\right] \\
F_{k}^{\text {sat }} & \frac{2}{3} E_{k}^{3 / 2} / \ln \left[\gamma\left(\frac{2 \tau}{176}\right) \sqrt{\frac{\pi}{2}} / 4.605\right] \\
& \frac{2}{3} E_{k}^{3 / 2} / \ln \left[\gamma\left(\frac{2 \tau}{176}\right)\left(\pi \rho V_{0}\right) / N_{a p}\right]
\end{array}
$$

Table 3.7.1. Scaling Laws and formulas for the simple-atom model for circular polarization. $\mathrm{K}_{1}$ is the Modified Bessel Function of the Second Kind of order 1. $F_{k+1}^{\text {thres }}$ and $F_{k}^{\text {sat }}$ are defined for $p_{\text {thr }}=p s a t=.01$ 
$\Gamma$

$$
\gamma\left(\frac{2}{\pi}\right)\left(\frac{1}{F_{k 0}}\right) K_{0}\left(\frac{1}{F_{k 0}}\right)
$$

$\gamma$

$$
6 \omega_{0} E_{k}
$$

$\phi$

$$
\gamma\left(\frac{2 \tau}{1.76}\right) \exp \left(-\frac{1}{F_{k 0}}\right)
$$

$N_{k+1}$

$$
\gamma\left(\frac{2 \tau}{1.76}\right)\left(2 \rho V_{0}\right)\left[3 \mathcal{F}_{k 0}{ }^{2} K_{1}\left(\frac{1}{\mathcal{F}_{k 0}}\right)+\mathcal{F}_{k 0} K_{0}\left(\frac{1}{\mathcal{F}_{k 0}}\right)+2 K i_{1}\left(\frac{1}{\mathcal{F}_{k 0}}\right)\right]
$$

$F_{k+1}^{\text {thres }}$

$$
\frac{2}{3} E_{k}^{3 / 2} / \ln \left[\gamma\left(\frac{2 \tau}{1.76}\right) / .01\right]
$$

$F_{k}^{\text {sat }}$

$$
\frac{2}{3} E_{k}^{3 / 2} / \ln \left[\gamma\left(\frac{2 \tau}{176}\right) / 4.605\right]
$$

$\mathcal{F}_{k+1}^{\text {appear }}$

$$
\frac{2}{3} E_{k}^{3 / 2} / \ln \left[\gamma\left(\frac{2 \tau}{1.76}\right)\left(2 \rho V_{0}\right)\left(2 \sqrt{\frac{\pi}{2}}\right) / N_{a p}\right]
$$

Table 3.7.2. Scaling Laws and formulas for the cycle-averaged simple atom model for linear polarization. $\mathrm{K}_{0}$ is the Modified Bessei Functions of the Second Kind of order $0 . \mathrm{Ki}_{1}$ is the repeated integral of $\mathrm{K}_{0}$ of order $1 . F_{k+1}^{\text {thres }}$, and $F_{k}^{\text {sat }}$ are defined for $p_{t h r}=p s a t=.01$ 


$$
\begin{array}{ll}
\Gamma & \gamma\left(\frac{1}{F_{k 0}}\right)^{P+1} \exp \left(-\frac{1}{F_{k 0}}\right) \\
\gamma & \omega_{0} \tilde{C}_{n !}{ }^{2} G_{l m} \frac{E_{k}}{2} \\
\phi & \gamma\left(\frac{2 \tau}{1.76}\right)\left(-\frac{\partial}{\partial \beta}\right)^{P} \frac{1}{\beta}\left(\frac{\beta}{F_{k 0}}\right) K_{1}\left(\frac{\beta}{F_{k 0}}\right) \\
N_{k+1} & \gamma\left(\frac{2 \tau}{176}\right)\left(\pi \rho V_{0}\right)\left(-\frac{\partial}{\partial \beta}\right)^{p} \frac{1}{\beta}\left(\frac{\mathcal{F}_{k 0}}{\beta}+\frac{\mathcal{F}_{k 0}{ }^{2}}{\beta^{2}}+\frac{\mathcal{F}_{k 0}{ }^{3}}{\beta^{3}}\right) \exp \left(-\frac{\beta}{\mathcal{F}_{k 0}}\right) \\
F_{k+1}^{\text {thres }} & \frac{2}{3} E_{k}^{3 / 2} / \ln \left[\gamma\left(\frac{2 \tau}{1.76}\right) \sqrt{\frac{\pi}{2}} / .01\right] \\
F_{k}^{\text {sat }} & \frac{2}{3} E_{k}^{3 / 2} / \ln \left[\gamma\left(\frac{2 \tau}{176}\right) \sqrt{\frac{\pi}{2}} / 4.605\right] \\
\mathcal{F}_{k+1}^{\text {appear }} & \frac{2}{3} E_{k}^{3 / 2} / \ln \left[\gamma\left(\frac{2 \tau}{176}\right)\left(\pi \rho V_{0}\right) / N_{a p}\right]
\end{array}
$$

Table 3.7.3. Scaling Laws and formulas for the complex-atom or ADK model for circular polarization. $P=2 n-|m|-2 . F_{k+1}^{\text {thres }}$ and $F_{k}^{\text {sat }}$ are defined for $p_{\text {thr }}=p$ sat $=.01$ 


$$
\begin{array}{lll}
\Gamma & & \gamma\left(\frac{2}{\pi}\right)\left(-\frac{\partial}{\partial \beta}\right)^{P+1} \frac{1}{\beta}\left(\frac{\beta}{F_{k 0}}\right) K_{0}\left(\frac{\beta}{F_{k 0}}\right) \\
\gamma & \omega_{0} \tilde{C}_{n l}^{2} G_{l m} \frac{E_{k}}{2} \\
\phi & \gamma\left(\frac{2 \tau}{1.76}\right)\left(-\frac{\partial}{\partial \beta}\right)^{P} \frac{1}{\beta} \exp \left(-\frac{\beta}{F_{k 0}}\right) \\
N_{k+1} & \gamma\left(\frac{2 \tau}{176}\right)\left(2 \rho V_{0}\right)\left(-\frac{\partial}{\partial \beta}\right)^{p} \frac{1}{\beta}\left[3\left(\frac{\mathcal{F}_{k 0}}{\beta}\right)^{2} K_{1}\left(\frac{\beta}{\mathcal{F}_{k 0}}\right)+\frac{\mathcal{F}_{k 0}}{\beta} K_{0}\left(\frac{\beta}{\mathcal{F}_{k 0}}\right)+2 K_{1}\left(\frac{\beta}{\mathcal{F}_{k 0}}\right)\right] \\
& \frac{2}{3} E_{k}^{3 / 2} / \ln \left[\gamma\left(\frac{2 \tau}{176}\right) / .01\right] \\
F_{k+1}^{\text {thres }} & \frac{2}{3} E_{k}^{3 / 2} / \ln \left[\gamma\left(\frac{2 \tau}{1.76}\right) / 4.605\right] \\
& \frac{2}{3} E_{k}^{3 / 2} / \ln \left[\gamma\left(\frac{2 \tau}{176}\right)\left(2 \rho V_{0}\right)\left(2 \sqrt{\frac{\pi}{2}}\right) / N_{a p}\right]
\end{array}
$$

Table 3.7.4. Scaling Laws and formulas for the cycleaveraged complex atom or ADK model for linear polarization. $F_{k+1}^{\text {thre }}$ and $F_{k}^{s a t}$ are defined for $p_{t h r}=$ psat $=.01$ 


\begin{tabular}{ll}
$\Gamma$ & $\gamma F_{k 0}^{2 n}$ \\
$\gamma$ & $6 \omega_{0} E_{k}(2 n+1)^{2 n+1} e^{-(2 n+1)}$ \\
$\phi$ & $\gamma\left(\frac{\tau}{176}\right) \mathrm{B}(1 / 2, n) F_{k 0}^{2 n}$ \\
$N_{k+1}$ & $\gamma\left(\frac{\tau}{176}\right) \mathrm{B}(1 / 2, n) \frac{3 \pi}{n} \frac{(2 n-5) ! !}{(2 n-4) ! !} \rho V_{0} \mathcal{F}_{k 0}^{2 n}$ \\
$F_{k+1}^{\text {thres }}$ & $\frac{2}{3} E_{k}^{3 / 2} /\left(\gamma\left(\frac{\tau}{176}\right) \mathrm{B}(1 / 2, n) / .01\right)^{\frac{1}{2 n}}$ \\
$F_{k}^{\text {sat }}$ & $\frac{2}{3} E_{k}^{3 / 2} /\left(\gamma\left(\frac{\tau}{1.76}\right) \mathrm{B}(1 / 2, n) / 4.605\right)^{\frac{1}{2 n}}$ \\
& $\frac{2}{3} E_{k}^{3 / 2} /\left(\gamma\left(\frac{\tau}{176}\right) \mathrm{B}(1 / 2, n) \frac{3 \pi}{n} \frac{(2 n-5) ! !}{(2 n-4) ! !} \rho V_{0} / N_{a p}\right)^{\frac{1}{2 n}}$ \\
\hline$F_{k+1}^{a \text { appear }}$ &
\end{tabular}

Table 3.7.5. Scaling Laws and formulas for the multiphoton model. $\mathrm{n}$ is the number of photons required to ionize the atom. $\gamma$ was chosen so that the MPI rate law joins smoothly to the Simple Atom rate law. $\mathrm{B}(u, v)=\Gamma(u) \Gamma(v) / \Gamma(u+v)$, Equations. (3.512.2) and (8.384.1) of Reference[43]. $F_{k+1}^{\text {thres }}$ and $F_{k}^{\text {sat }}$ are defined for $p_{\text {thr }}=p$ sat $=.01$ 


\begin{tabular}{|c|c|c|c|c|}
\hline Polarization & Species & $I^{\text {thresh }}\left(\mathrm{W} / \mathrm{cm}^{2}\right)$ & $I^{\text {sat }}\left(\mathrm{W} / \mathrm{cm}^{2}\right)$ & $I^{\text {sat }} / I^{\text {thresh }}$ \\
\hline \multirow[t]{6}{*}{ Linear } & $\mathrm{He}^{+1}$ & $4.00 \times 10^{14}$ & $9.34 \times 10^{15}$ & 23.3 \\
\hline & $\mathrm{Ne}^{+1}$ & $2.35 \times 10^{14}$ & $3.23 \times 10^{15}$ & 13.7 \\
\hline & $\mathrm{Ar}^{+1}$ & $9.24 \times 10^{13}$ & $1.02 \times 10^{15}$ & 11.0 \\
\hline & $\mathrm{He}^{+2}$ & $3.68 \times 10^{15}$ & N/A & N/A \\
\hline & $\mathrm{Ne}^{+2}$ & $1.36 \times 10^{15}$ & $1.07 \times 10^{16}$ & 7.87 \\
\hline & $\mathrm{Ar}^{+2}$ & $4.28 \times 10^{14}$ & $2.88 \times 10^{15}$ & 6.73 \\
\hline \multirow[t]{6}{*}{ Circular } & $\mathrm{He}^{+1}$ & $7.11 \times 10^{14}$ & $1.58 \times 10^{16}$ & 22.2 \\
\hline & $\mathrm{Ne}^{+1}$ & $4.22 \times 10^{14}$ & $5.61 \times 10^{15}$ & 13.3 \\
\hline & $\mathrm{Ar}^{+1}$ & $1.65 \times 10^{14}$ & $1.74 \times 10^{15}$ & 10.5 \\
\hline & $\mathrm{He}^{+2}$ & $6.60 \times 10^{15}$ & N/A & N/A \\
\hline & $\mathrm{Ne}^{+2}$ & $2.50 \times 10^{15}$ & $1.85 \times 10^{16}$ & 7.4 \\
\hline & $\mathrm{Ar}^{+2}$ & $7.72 \times 10^{14}$ & $4.98 \times 10^{15}$ & 6.45 \\
\hline
\end{tabular}

Table 3.7.6 The ADK threshold and saturation irradiances for linear and circular polarization. The irradiances are based on the equations of Tables 3.7.3 and 3.74 using the effective principle quantum numbers of the ground state configurations. The $m$ quantum numbers are set to 0 . The pulse width used is $125 \mathrm{fs}$. 


\subsection{Characteristics of Sequential Tunneling}

Analytic solutions to the rate equations for sequential tunneling exist for several models. These solutions give explicitly the dependence of the yields on parameters of the system. For simple tunneling, Equation (3.5.1) describes the number of ions produced versus the normalized peak field, $\mathcal{F}_{k 0}$, for irradiances below the saturation defined at $p_{s a t}=01$. This equation reveals general characteristics of tunneling yields that are important in this work. First, the yield is initially a high-order function of $\mathcal{F}_{k 0}$ that rolls off to a constant power at high $\mathcal{F}_{k 0}$. In this particular case the power is $\mathcal{F}_{k 0}^{3}$ and agrees with the high-irradiance dependence given by Equation (3.6.2), but that agreement is not guaranteed. Second, the yield exhibits no maxima, minima or inflection points. Thus completely sequential tunneling ionization will produce yields exhibiting only a single saturation. This is true even when the high-irradiance limit is included. Also the $I^{\text {sat }} / I^{\text {thresh }}$ comparison indicates that this characteristic sequential ionization yield curve steepens at threshold with increasing ionization potential; yet beyond volume saturation the curves should be parallel for all charge states (on a $\log -\log$ scale). The yield contains no local structure and any such structure indicates deviations from the sequential assumption or the tunneling assumption. 


\section{CHAPTER 4 - THE LASER SYSTEMS}

\subsection{Introduction}

Two laser systems produced the optical fields used in this work. The first laser system is an amplified colliding-pulse mode-locked dye laser (CPM) operating at $614 \mathrm{~nm}$. The CPM laser system produces pulses with energies up to $2.5 \mathrm{~mJ}$ in 120 fs. The second system is an amplified titanium-sapphire ( $\left.\mathrm{Ti}: \mathrm{Al}_{2} \mathrm{O}_{3}\right)$ laser operating at $800 \mathrm{~nm}$. The $\mathrm{Ti}: \mathrm{Al}_{2} \mathrm{O}_{3}$ laser system produces pulses with energies of up to $60 \mathrm{~mJ}$ in 130 femtoseconds.

\subsection{Description of Laser Pulses and Irradiance}

In these experiments the critical parameters of the laser pulses are the peak electric field, the pulse width and the ellipticity. The ellipticity is directly measurable. I relate the peak field to the irradiance through Equation (2.7.7). If the laser pulse is separable into a function of space, $F(r, \theta, z)$, and a function of time, $T(t)$, the irradiance of the pulse may be represented as

$$
I(r, z, t)=I_{0} F(r, \theta, z) T(t)
$$

At $\mathrm{z}=0$, the focus of the laser, the energy in the pulse, $E_{p}$, is

$$
E_{p}=I_{0} \int_{0}^{\infty} \int_{0}^{2 \pi} F(r, \theta, 0) r d r d \theta \int_{-\infty}^{\infty} T(t) d t
$$

Thus the peak irradiance of the pulse at the focus is

$$
I_{0}=\frac{E_{p}}{\int_{0}^{\infty} \int_{0}^{2 \pi} F(r, \theta, 0) r d r d \theta \int_{-\infty}^{\infty} T(t) d t} .
$$

Now let the two-dimensional integral in space be 


$$
A=\int_{0}^{-2 \pi} \int_{0}^{2 \pi} F(r, \theta, 0) r d r d \theta
$$

The peak irradiance becomes

$$
I_{0}=\frac{E_{p}}{A \int_{-\infty}^{\infty} T(t) d t}
$$

While the spatial profiles for both lasers are modeled as Gaussian beams with

$$
F(r, \theta, z)=F(r, z)=\left[\frac{1}{1+\left(\lambda z / \pi w_{0}^{2}\right)}\right] \exp \left[\frac{-2 r^{2}}{w_{0}^{2}\left[1+\left(\lambda z / \pi w_{0}{ }^{2}\right)\right]}\right],
$$

I obtain $A$ by direct numerical integration of an image of the focal profile. $E_{p}$ is measured using a thin beamsplitter and a Molectron JD2000 energy meter. Autocorrelation techniques that I will discuss later provide the knowledge necessary to obtain an estimate of $\int_{-\infty}^{\infty} T(t) d t$.

Now consider a pulse with a squared hyperbolic-secant temporal profile,

$$
T(t)=\operatorname{sech}^{2}\left(2 t / \tau_{p}\right)
$$

Integrating over the this profile

$$
\int_{-\infty}^{\infty} T(t) d t=\tau_{p}
$$

The pulse width, $\tau_{p}$, is related to the measured full-width-at-half-maximum pulse width, $\tau$, by $\tau_{p}=1.135 \tau$. The peak irradiance for a pulse with a squared hyperbolic-secant temporal profile is

$$
I_{0}=\frac{E_{p}}{1.135 \tau A} .
$$


For a pulse with a Gaussian temporal profile with a FWHM pulse width $\tau$,

$$
T(t)=\exp \left(-\left(2 \sqrt{\ln 2} \frac{t}{\tau}\right)^{2}\right)
$$

and

$$
\int_{-\infty}^{\infty} T(t) d t=1064 \tau .
$$

Thus the peak irradiance for a pulse with a Gaussian temporal profile is given by

$$
I_{0}=\frac{E_{p}}{1064 \tau A} .
$$




\subsection{The Colliding Pulse Mode-Locked Dye Laser System}

Figure 4.3.1 is a diagram of the CPM laser system. The oscillator for the CPM laser system is a Clark Instrumentation, Inc. CPM-1 Colliding Pulse Modelocked Dye laser. This oscillator is a ring dye-laser that passively mode locks[4549]. The CPM-1 produces 140 femtosecond pulses of $612-616 \mathrm{~nm}$ light at a $90 \mathrm{MHz}$ repetition rate. A series of three amplifiers-a multi-pass dye cell, a $6 \mathrm{~mm}$ Bethune cell[50], and a $20 \mathrm{~mm}$ Bethune cell-amplifies the laser pulse energy to 2-3 mJ. The amplifier chain operates at 10 pulses-per-second. The second harmonic of a single Nd:YAG laser pumps all three amplifiers. Spatial

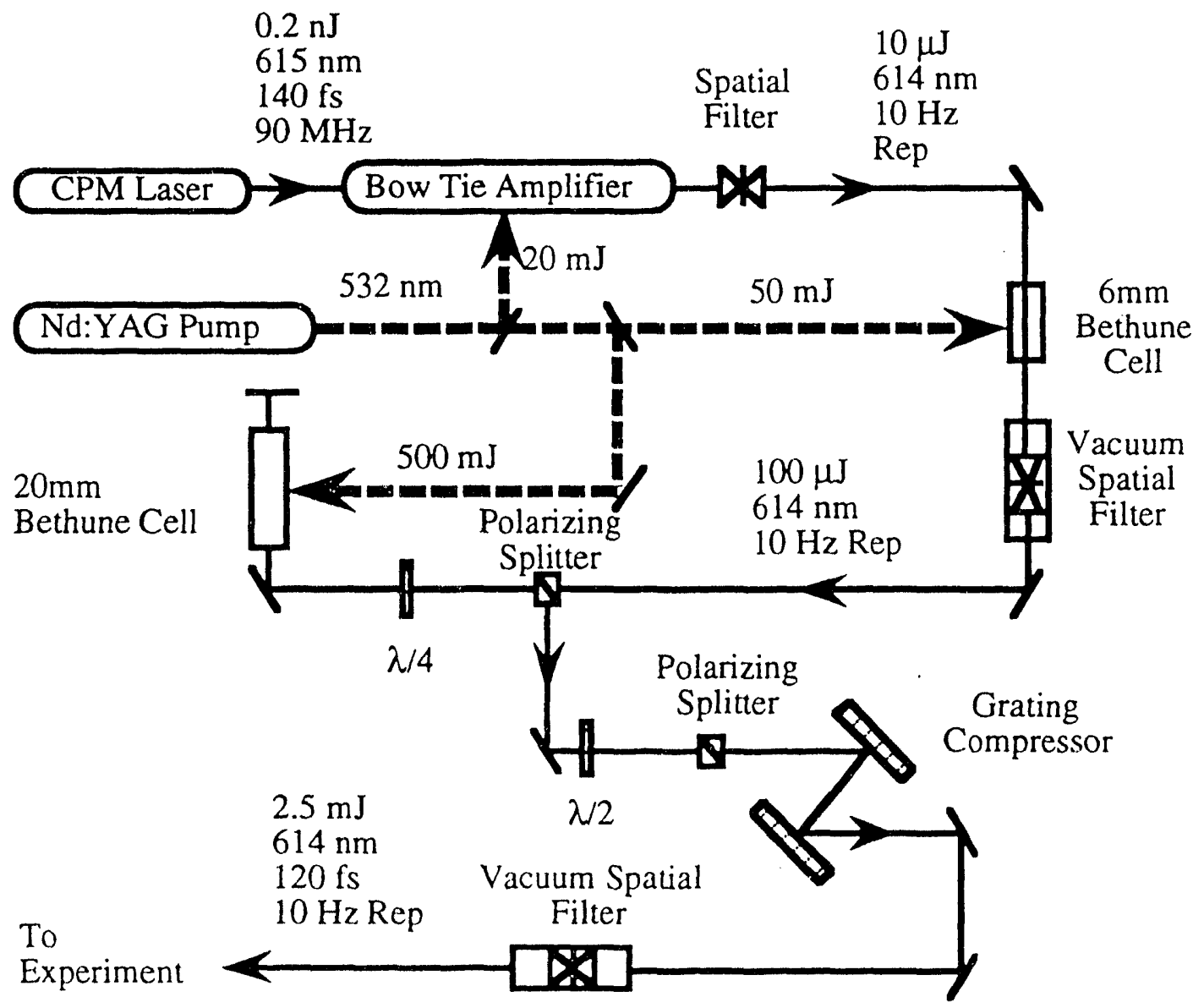

Figure 4.3.1 The colliding-pulse mode-locked dye laser system. 
filters placed after the amplifier stages reduce the amplified spontaneous emission (ASE) providing an estimated prepulse energy contrast ratio near $10^{4}$. A $\lambda / 2$ waveplate and a polarizing beamsplitting cube control the output energy over two orders of magnitude while maintaining greater than $99 \%$ linear polarization. A grating-pair compressor, placed just before the final output, counters dispersion broadening and provides further pulse compression. The final output of the laser system is a near flat-top beam $2 \mathrm{~cm}$ in diameter. The output pulse width is $120 \mathrm{fs}$ with a pulse energy of $2.5 \mathrm{~mJ}$. 


\subsection{Autocorrelation Measurement of the CPM Pulse Width.}

A standard single-shot autocorrelator monitored the temporal pulse width of the laser during the data runs. The autocorrelator uses non-collinear second harmonic generation (NSHG) in a KDP crystal to produce a spatial autocorrelation signal of the irradiance of the pulse[51-53]. A reticon diode array and a Hewlett Packard 54502 digitizing oscilloscope record the spatial profile of the NSHG signal. Figure 4.4.1 shows a schematic of the autocorrelator.

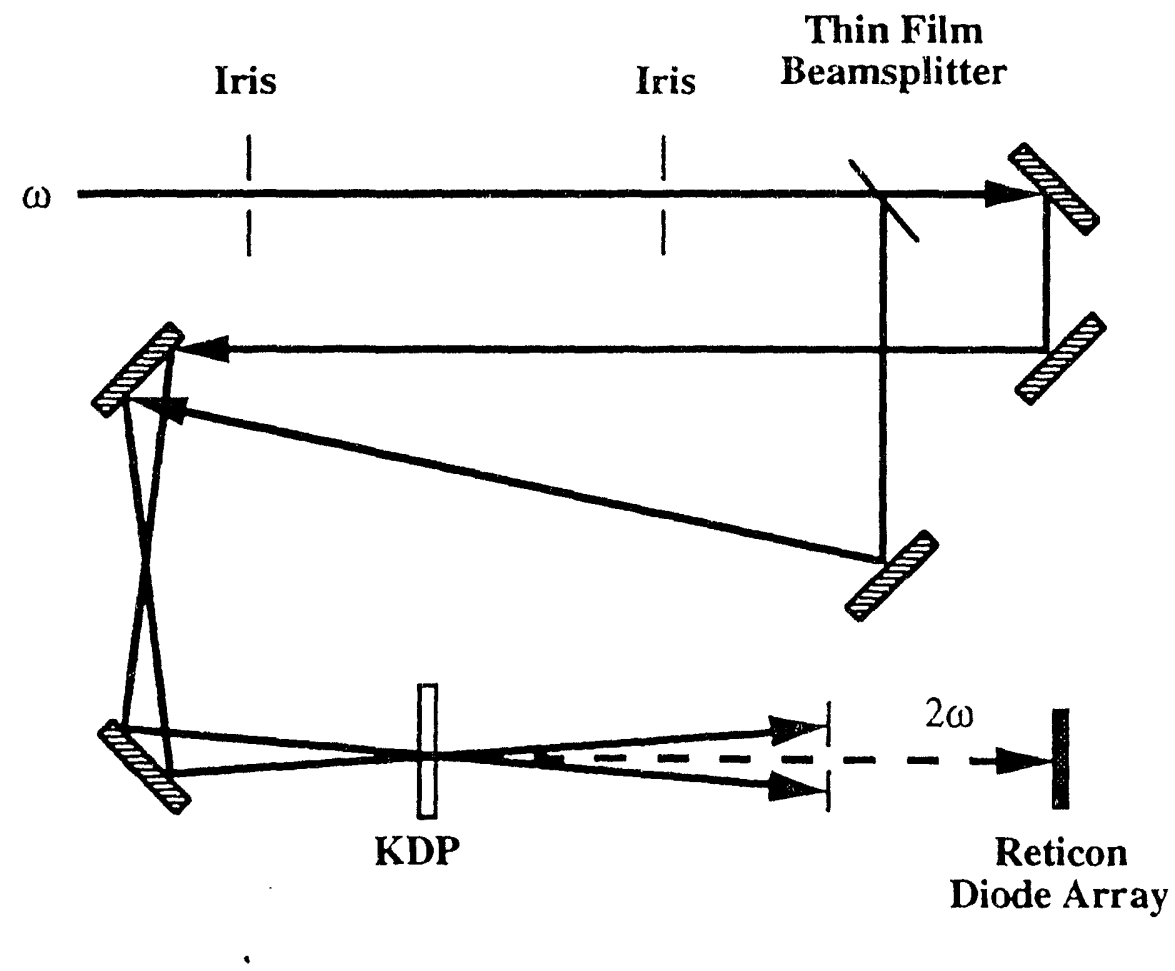

Figure 4.4.1 Single-shot autocorrelator uses nonlinear second harmonic generation to monitor pulse width.

A thin beamsplitter divides the laser pulse in two. These pulses cross in a KDP crystal. Where the two pulses overlap spatially and temporally, second harmonic generation occurs. Assume that the two pulses are spatially uniform and have equal temporal irradiance profiles, $I(t)$. For pulses propagating through the crystal at angles $\phi / 2$ and $-\phi / 2$ with respect to the $z$-axis (z-axis parallel to the 
propagation direction), the detector records a second harmonic signal at a point off the $z$-axis a distance $x$ that is proportional to the autocorrelation function $G_{2}\left(2 \tau_{d}\right)$ of the incident pulse[53].

$$
G_{2}\left(2 \tau_{d}\right)=\int_{-\infty}^{\infty} I\left(t+\tau_{d}\right) I\left(t-\tau_{d}\right) d t
$$

where the delay $\tau_{d}$ is

$$
\tau_{d}=\frac{n x}{c} \sin \phi / 2
$$

Here $n$ is the index of refraction in the KDP crystal, and $\mathrm{c}$ is the velocity of light. The autocorrelator was calibrated by introducing a known delay, $\Delta t_{0}$, in one of the two beams, and observing the shift, $\Delta \mathrm{x}_{0}$ of the second harmonic signal along the $x$-axis[51, 53]. The shift is proportional to the time delay and gives the calibration scale for the autocorrelation signal. I introduced delays using a micrometer stage and using a thin quartz slide that I calibrated using a scanning autocorrelator. The two methods produced equal calibration factors. The NSHG signal is not the second-order autocorrelation function, $G_{2}\left(\tau_{d}\right)$, but is the function $G_{2}\left(2 \tau_{d}\right)$. The full width at half-maximum of the incident pulse, $\tau$, is related to the full width at half-maximum of the spatial autocorrelation signal, $x_{a}$, by

$$
\tau=\frac{x_{a}}{K} \frac{\Delta t_{0}}{\Delta x_{0}}
$$

Here $\mathrm{K}$ is a shape factor that depends on the incident pulse shape. $\mathrm{K}$ is 1.55 for hyperbolic-secant-squared pulse and 1.414 for a Gaussian pulse[54]. For the CPM laser the predicted pulse shape is hyperbolic-secant squared[47]. While I tried several different pulse shapes to fit the autocorrelation signal, the hyperbolicsecant squared pulse shape fit the best. Figure 4.4 .2 shows a measured autocorrelation and a theoretical fit for a $120 \mathrm{fs}$ pulse. 


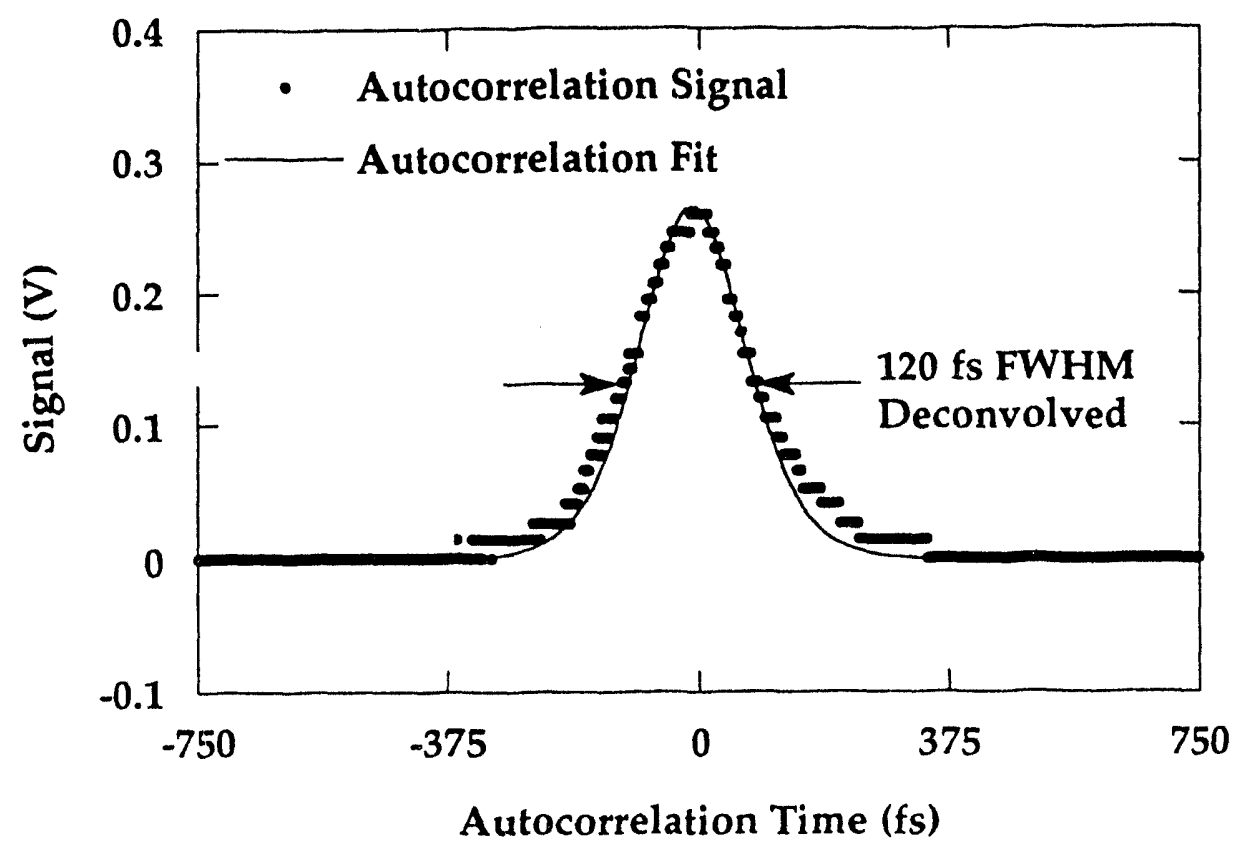

Figure 4.4.2 Single-single shot autocorrelation and a fitted autocorrelation for a hyperbolic-secant-squared pulse. The deconvolved width is 120 fs FWHM.

\subsection{CPM Laser Focal Properties}

Early in these experiments, measurements of the $\mathrm{He}^{+1}$ and $\mathrm{Xe}^{+1}$ appearance irradiances disagreed with the tunneling predictions. The error arose through straightforward application of Equation (4.2.9). I had measured the focal spot and modeled it using an Airy pattern. In this case,

$$
A=\pi w_{0}^{2} / 3.67
$$

and Equation (4.2.9) becomes 


$$
I_{0}=\frac{3.67 E_{p}}{1.135 \tau \pi w_{0}^{2}} .
$$

The error in using this form arose because I had used an 8-bit camera for the initial focal profile measurements and had observed a seemingly clean Airy pattern. I remeasured the focal profile by imaging the focal plane with a 14-bit camera. Insertion of a resolution target (Mil. Std. 150-A;5.1.1.7) at the focus provided the scale for the image, and the resolution of the measurements was $0.229 \mu \mathrm{m} / \mathrm{pixel}$. Figure 4.5.1 shows the type of focal profile that I obtained at the time using the $10-\mathrm{cm}$ focal length off-axis

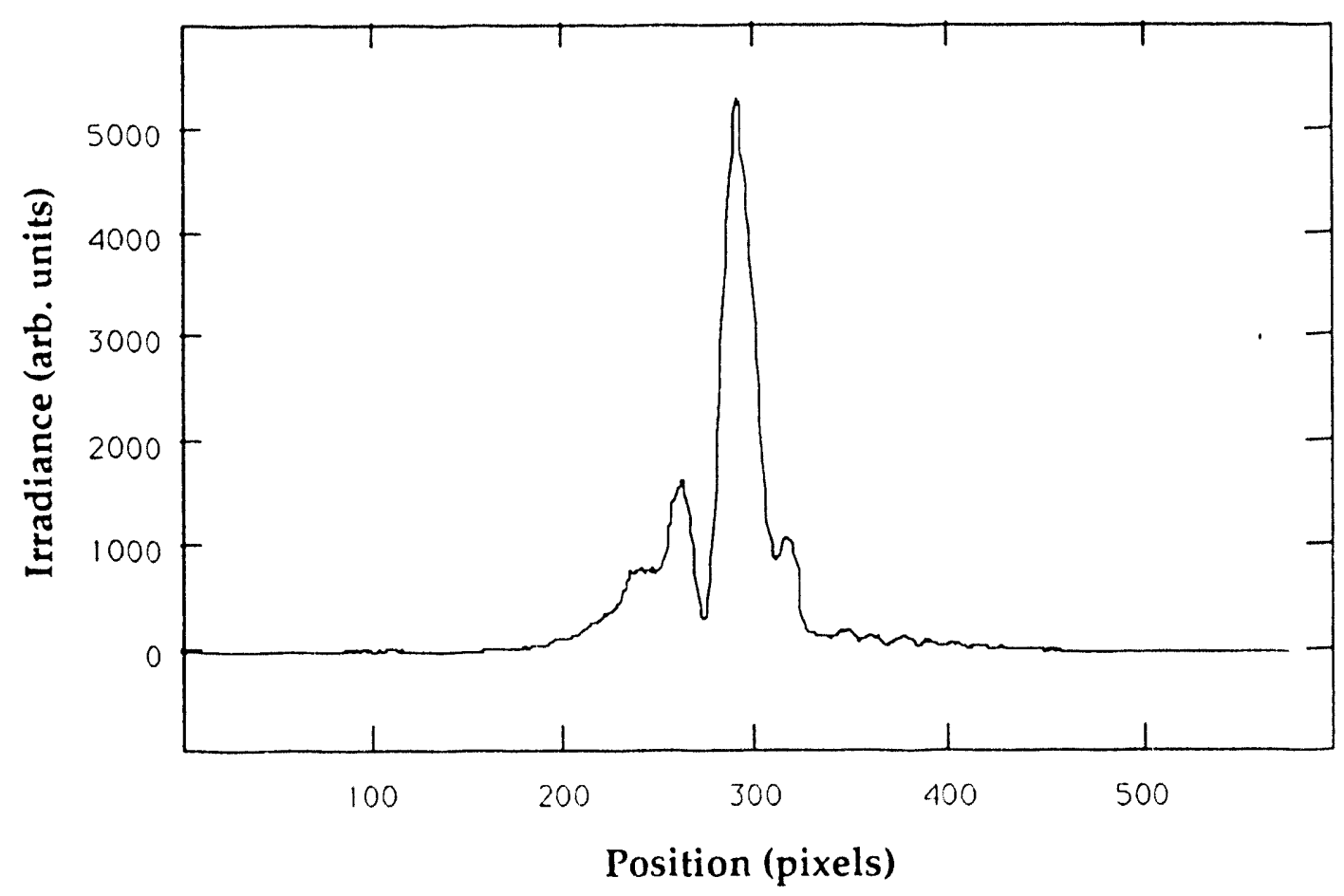

Figure 4.5.1 Initial profile at the focus of the $f / 5$ off-axis parabola. 
parabola used in the experiment. With a strong central spike and surrounding ring structure, the profile looks very similar to that obtained using the 8-bit camera and very much like an Airy pattern. The full-width at half-maximum is $4.5 \mu \mathrm{m}$. When, however, I viewed this profile on a logarithmic scale, I found that a large low-irradiance halo of energy around the central spike (see Figure 4.5.2) that had been invisible to the 8-bit camera. This halo contained most of the energy in the beam. Even worse, because the ion yields are sensitive functions of irradiance, the energy in the low-irradiance halo did not contribute to the

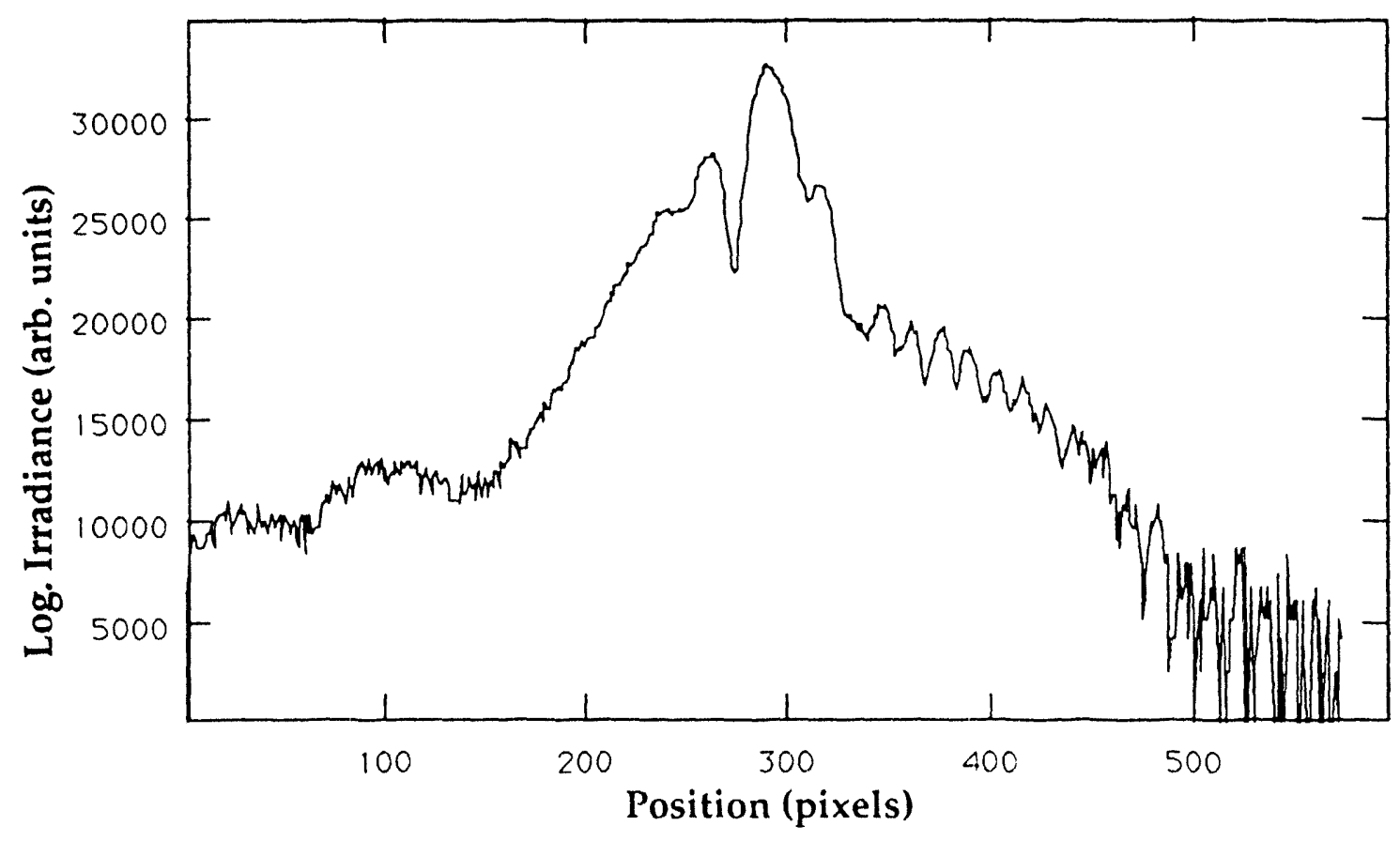

Figure 4.5.2 Initial profile at the focus of the $f / 5$ off-axis parabola viewed on a logarithmic scale. The low irradiance halo contained most of the energy in the beam. 
production of ions. The irradiance in the halo was generally too low to ionize the gas. This amounted to a loss of energy and produced the gross overestimation in the initial estimates of the irradiance.

The halo arose from uneven pumping and overfilling of the $20 \mathrm{~mm}$ Bethune cell and poor spatial filtering. These problems introduced high spatial frequencies in the beam that would not focus well. After correcting these problems, I obtained the focal profile shown in Figure 4.5.3.

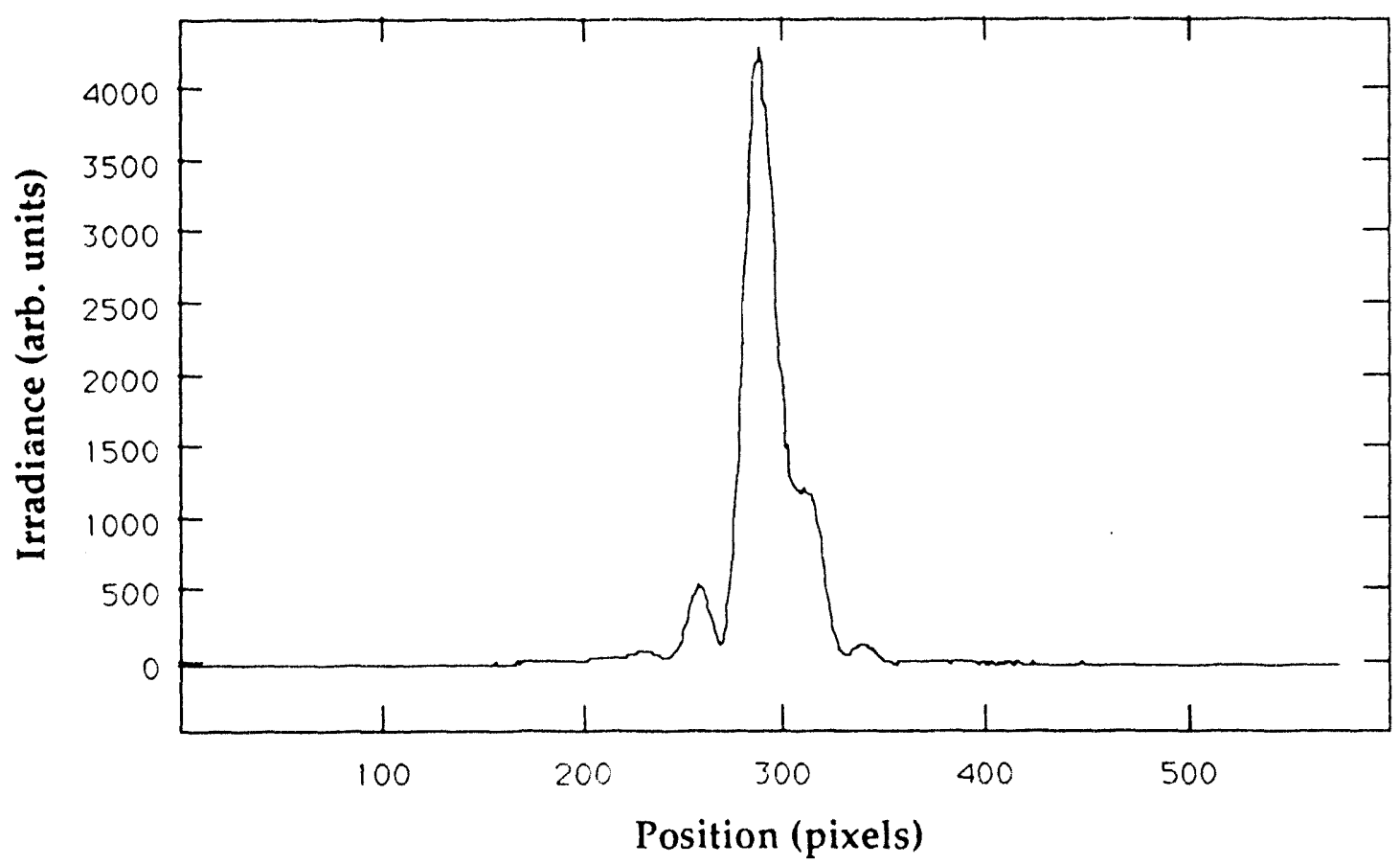

Figure 4.5.3 Final profile at the focus of the $f / 5$ off-axis parabola at $614 \mathrm{~nm}$. 
The focus still looks like an Airy pattern because the beam is roughly flattopped. Now, however, the logarithm of the focal profile (Figure 4.5.4) shows that the low-irradiance halo is greatly reduced. This is the profile used in the experiment. Because the halo still contains some energy ( $40 \%$ of total pulse energy) above that for an Airy pattern, the actual irradiance was calculated by numerically integrating the profile to obtain $A$. As shown later, the measured irradiance now agrees with the irradiance inferred from the ion yields (modeled for a Gaussian of the same full-width-at-half-maximum focal diameter) to within $30 \%$.

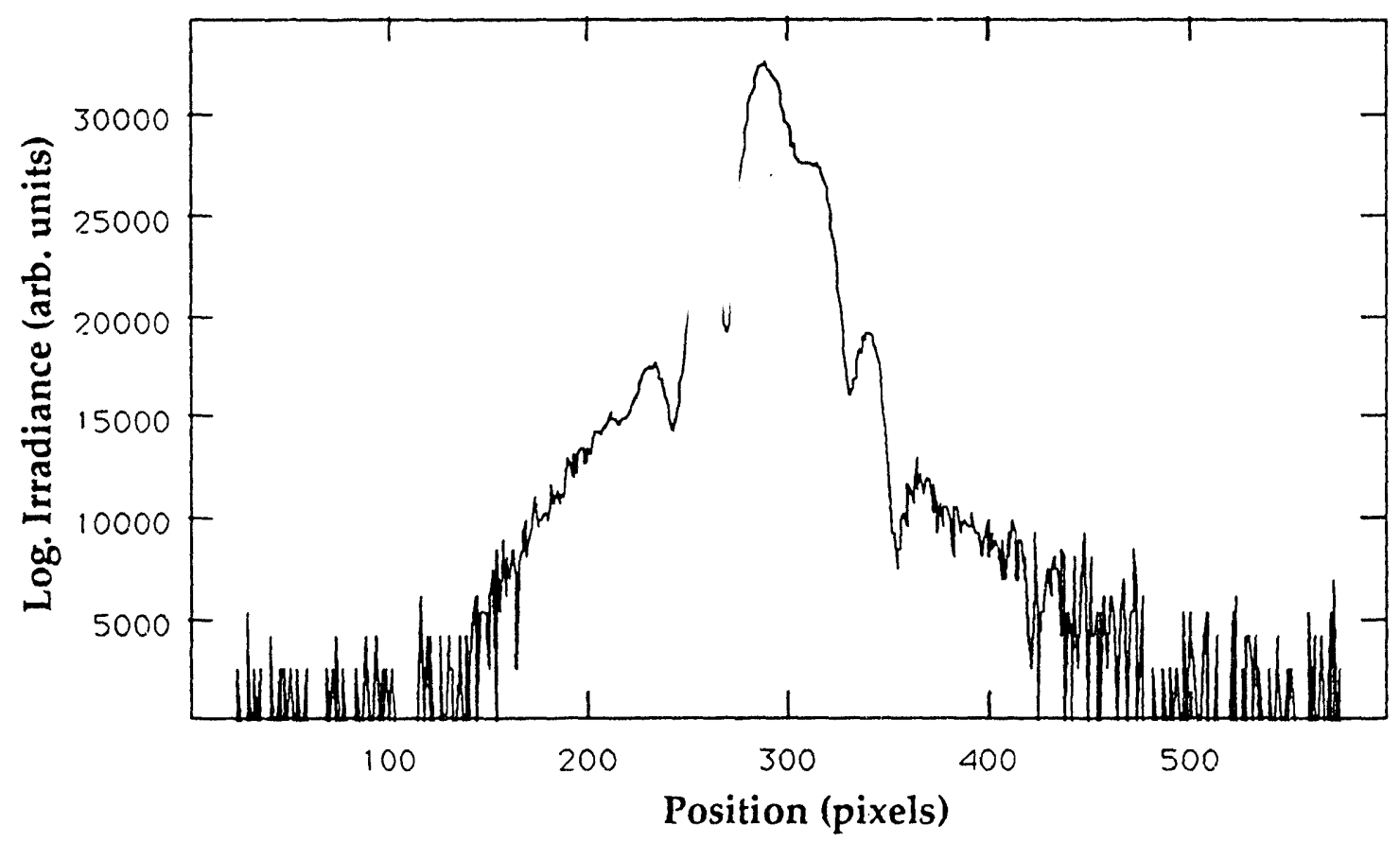

Figure 4.5.4 Final profile at the focus of the $f / 5$ off-axis parabola at $614 \mathrm{~nm}$ viewed on a logarithmic scale. 


\subsection{Spectral Measurements}

Although tunneling ionization is not strongly wavelength dependent, I measured the spectrum after changes of optics to watch for self-phase modulation that might affect the temporal profile without greatly altering the autocorrelation pulse width. Figure 4.3.8 shows a typical laser spectrum. For a transform-limited squared-hyperbolic-secant pulse the time-bandwidth product should be

$$
\Delta v \Delta t=\frac{\Delta \lambda \Delta t}{\lambda^{2}} c=0.3148
$$

where $c$ is the speed of light in a vacuum. The CPM laser system does not operate at the transform limit. With $\Delta \lambda=6 \mathrm{~nm}, \Delta t=120 \mathrm{fs}$ and $\lambda=614 \mathrm{~nm}$, the time

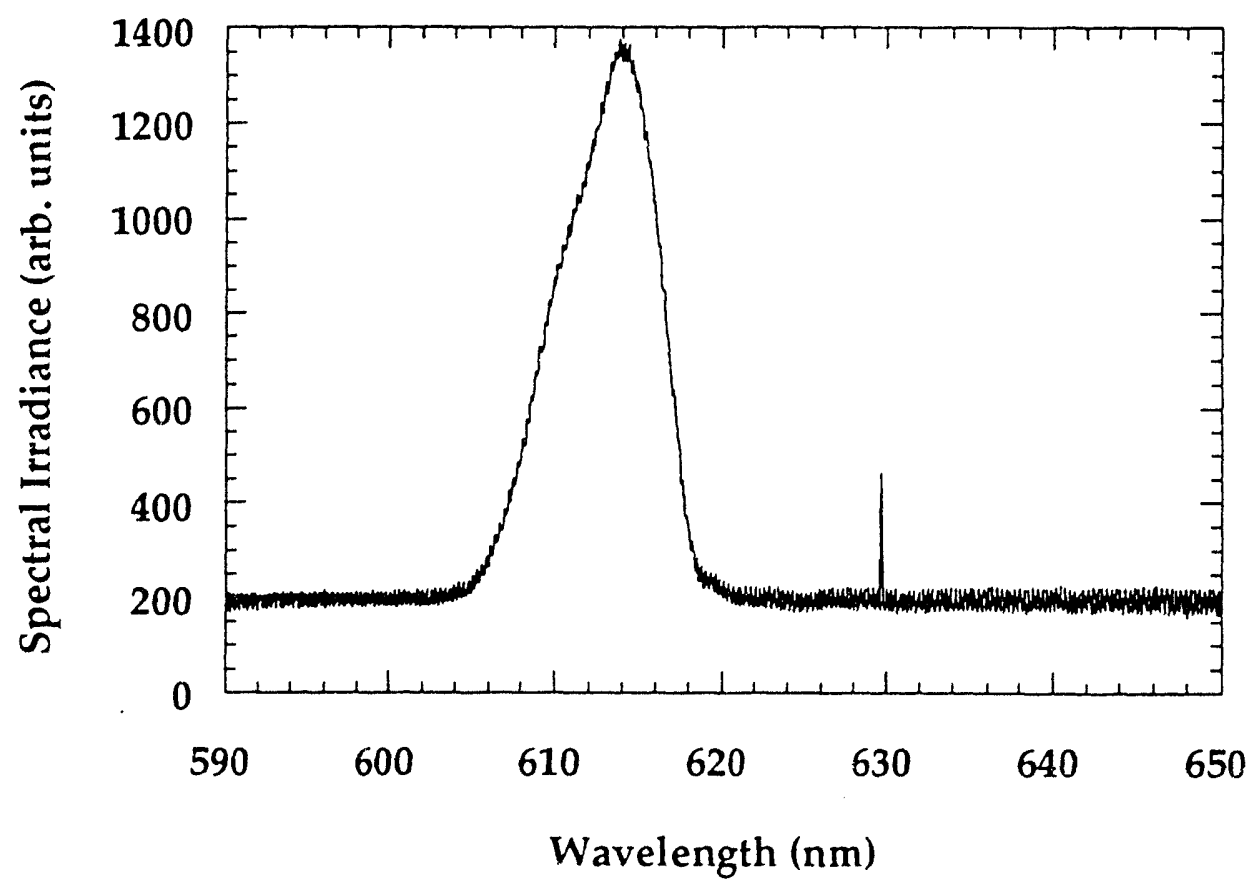

Figure 4.6.1 Typical CPM laser system spectrum. The spike at 630 $\mathrm{nm}$ is noise. 
bandwidth product is roughly 0.57 or twice the transform limit. Even without being transform limited the spectrum was sensitive enough to self phase modulation to be of use in the experiment. Figure 4.6.2 shows the spectrum obtained after a new set of lenses were installed in the final vacuum spatial filter. The pulse energy was roughly $2 \mathrm{~mJ}$. The new lenses were roughly $1 \mathrm{~cm}$ thick achromatic lenses made of BK-7. The spectrum exhibits both the spectral shifting and the broadening associated with self-phase modulation[55]. This case was extreme and was accompanied by broadening of the pulse width to $160 \mathrm{fs}$. I replaced the achromats with thin glass lenses and the temporal broadening and self-phase modulation disappeared.

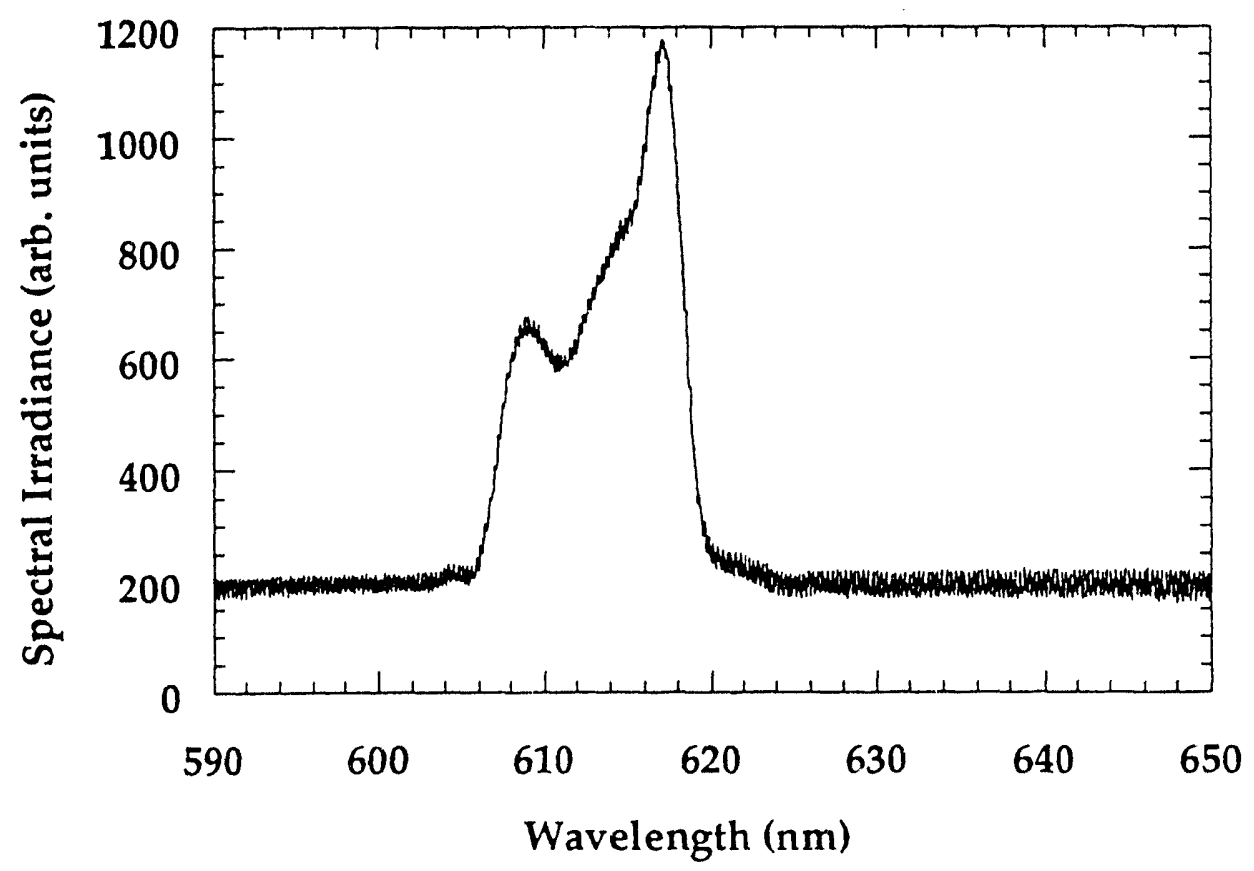

Figure 4.6.2 Spectrum of the CPM-laser pulses after installation of thick BK-7 lenses showing strong self-phase modulation. 


\subsection{Laser Polarization}

Because the ion yields are polarization dependent, I measured the polarization of the linearly and circularly polarized beams using the Stokes parameters. An explanation of the Stokes parameters is given in Appendix I. For the linear polarization measurements, $0 \leq|\varepsilon| \leq 005$. For the circular polarization measurements, $.948 \leq|\varepsilon| \leq 1$.

\subsection{The Titanium-Sapphire Laser System}

Figure 4.8.1 shows the titanium-sapphire $\left(\mathrm{Ti}_{\mathrm{Al}} \mathrm{Al}_{2} \mathrm{O}_{3}\right)$ laser system used in the experiment. In the system, an Ar-ion laser pumps a commercial mode-locked $\mathrm{Ti}: \mathrm{Al}_{2} \mathrm{O}_{3}$ oscillator. The oscillator produces near transform-limited 100-120 femtosecond pulses at a $82 \mathrm{MHz}$ repetition rate and produces 1.2 watts of average power. For these experiments the central wavelength was $800 \mathrm{~nm}$. A grating-pair stretches the pulse width to $440 \mathrm{ps}$. The stretched pulses couple into a regenerative amplifier off the surface of the Brewster-cut $\mathrm{Al}_{2} \mathrm{O}_{3}$ amplifier rod. The amplifier is pumped by a frequency-doubled Nd:YAG laser. The repetition rate of the amplifier chain is $10 \mathrm{~Hz}$. After 12 round trips in the regenerative amplifier, a single crystal Pockel cell placed between thin film dielectric polarizers switches a single pulse out. A second Pockel cell and polarizer pair then eliminate the low energy pulses that reflect off the switchout polarizer with each round trip inside the cavity. A final amplifier consisting of a double-passed $\mathrm{Ti}: \mathrm{Al}_{2} \mathrm{O}_{3}$ rod raises the uncompressed pulse energy to roughly 60 $\mathrm{mJ}$. A pair of gratings then compresses the laser pulses to $130 \mathrm{fs}$ with an energy of up to $30 \mathrm{~mJ}$. 


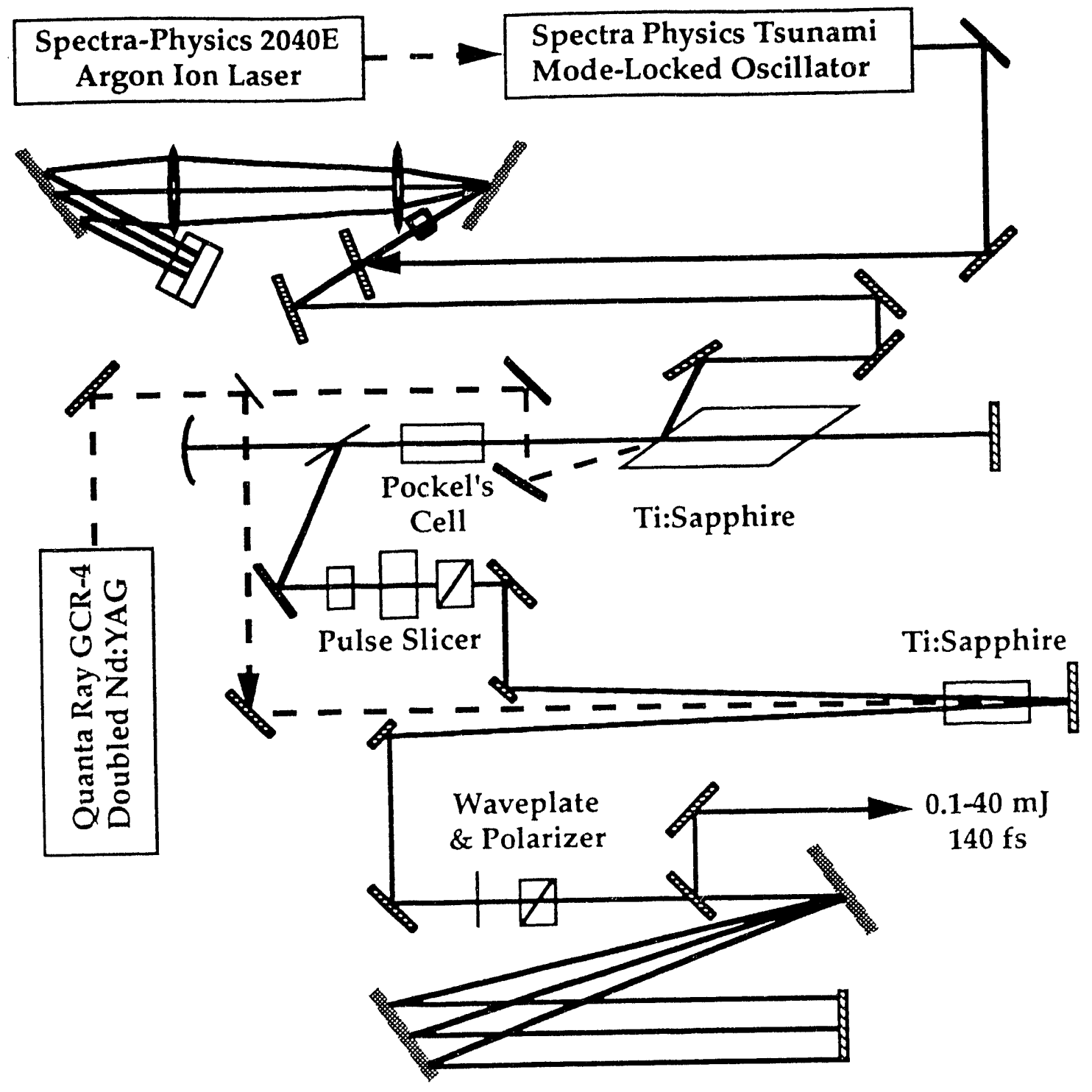

Figure 4.8.1 The titanium sapphire laser system produces $30 \mathrm{~mJ}$, 130-fs pulses at $800-\mathrm{nm}$.

The pulses were characterized as for the CPM laser system. The output pulses are temporally Gaussian with a FWHM pulse width of $130 \mathrm{fs}$. Figure 4.8.2 shows the measured autocorrelation signal versus the autocorrelation time and the fit for the autocorrelation of a Gaussian pulse. The spectrum of the laser is symmetric with FWHM of 7.5 to $8 \mathrm{~nm}$. The time-bandwidth 


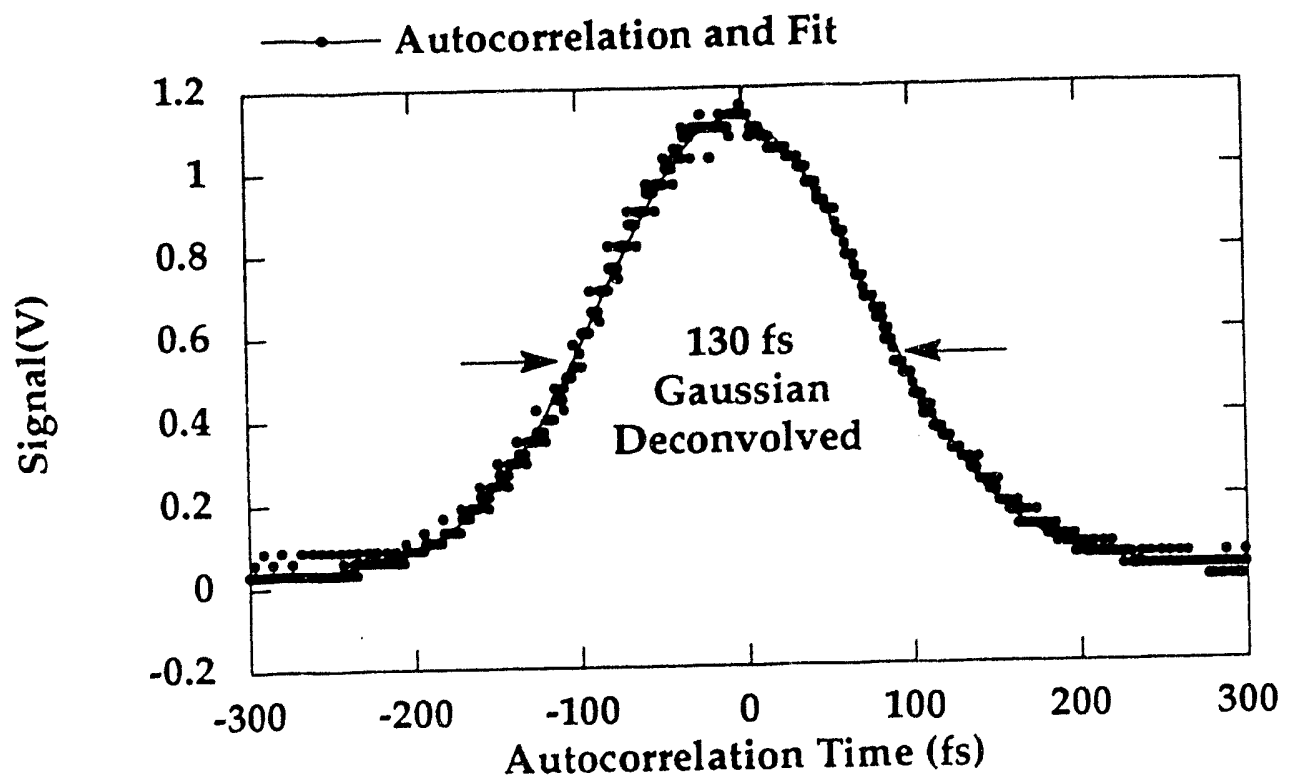

Figure 4.8.2 The autocorrelation of the $800-\mathrm{nm}$ laser pulse.

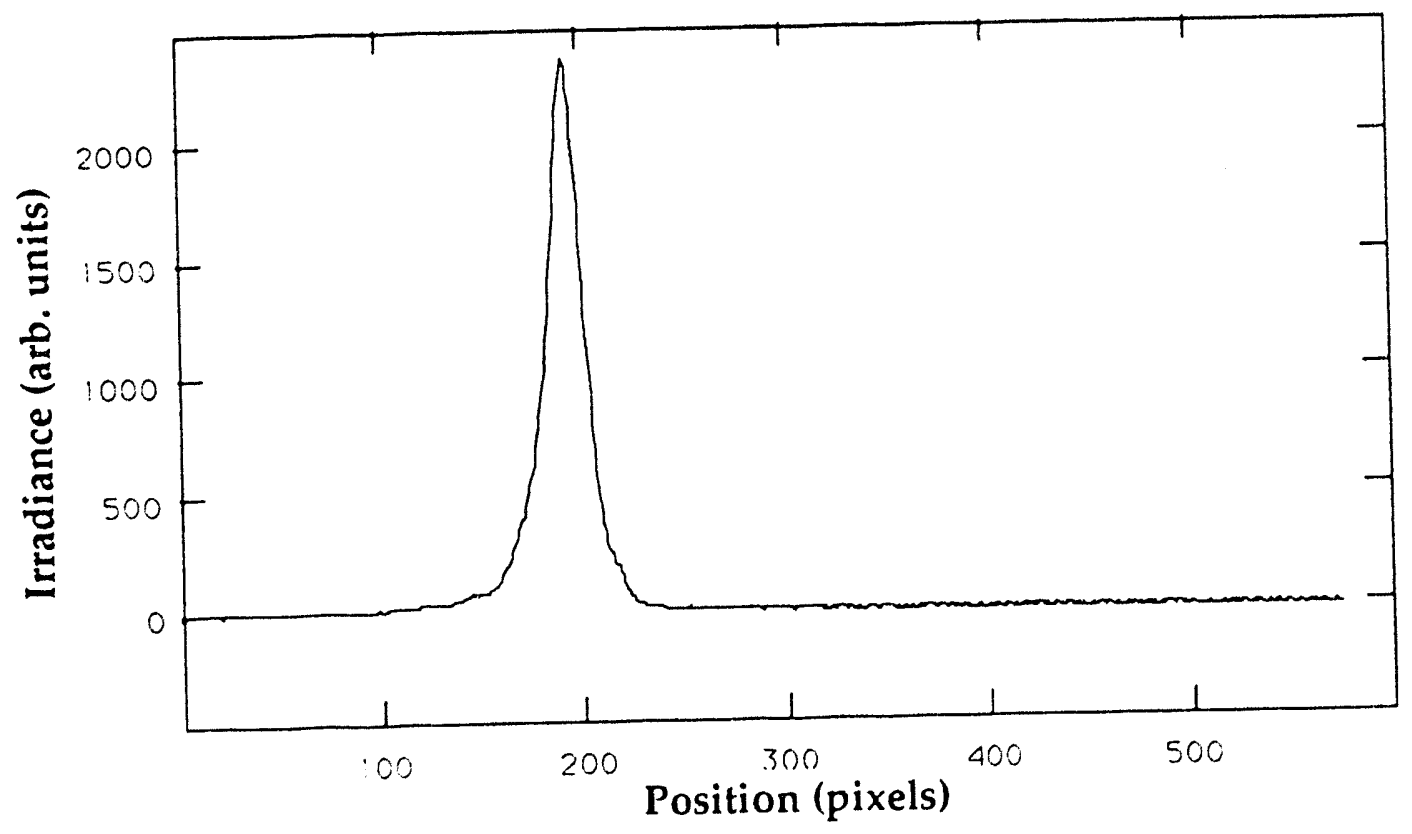

Figure 4.8.3 Focal profile of the $800-\mathrm{nm}$ laser pulse using the $10 \mathrm{~cm}$ focal length parabola. The position scale is $.458 \mu \mathrm{m} /$ pixel. 
product is $\approx .44$ to .5 . The transform-limited time-bandwidth product for a Gaussian is .44, so the pulses from the Ti:Al2O3 laser are nearly transform limited and should be nearly free of chirp and unusual temporal amplitude variations. The unfocused beam is spatially Gaussian with a FWHM diameter of 23 millimeters. Using the $10 \mathrm{~cm}$ focal length parabolic mirror that was used in the experiment, the beam focuses to a FWHM diameter of $9.5 \mu \mathrm{m}$. This is approximately 1.7 times the diffraction-limited spot size for the parabola. Figure 4.8.3 show the image of the focus of the beam. The image shows a strong central spike free of diffraction. The logarithm of the profile shown in Figure 4.8.4 reveals smaller low irradiance spatial wings than those that exist in the CPM focus.

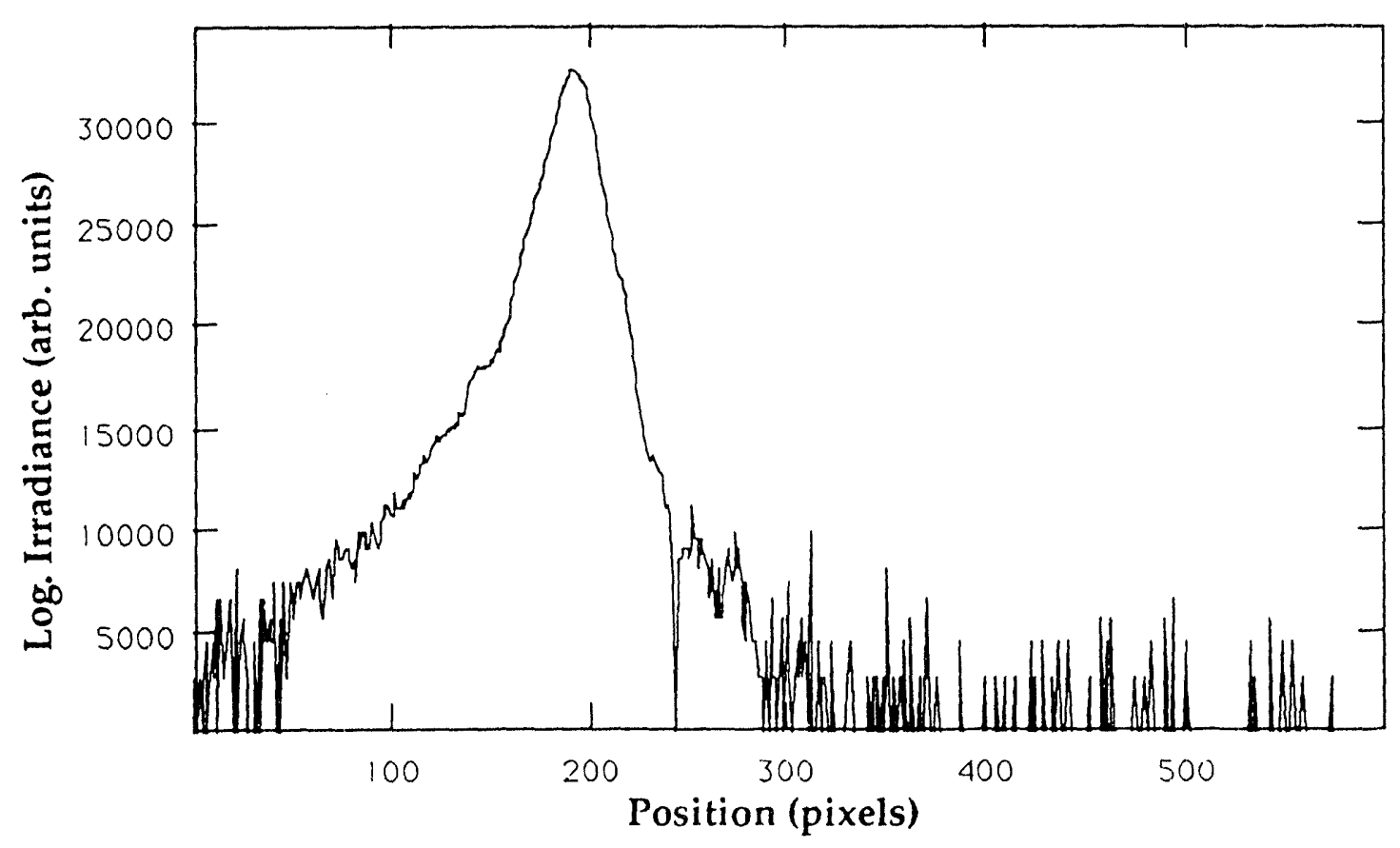

Figure 4.8.4 Logarithm of the focal profile at the focus of the $800-\mathrm{nm}$ laser pulse using the $10 \mathrm{~cm}$ focal length parabola. The position scale is $.458 \mu \mathrm{m} /$ pixel. The FWHM focal diameter is $9.5 \mu \mathrm{m}$. 


\subsection{Summary}

The two laser systems used in this work are well characterized. The first laser system is an amplified colliding-pulse mode-locked dye laser (CPM) operating at $614 \mathrm{~nm}$. The CPM laser system produces squared-hyperbolic secant pulses with energies up to $2.5 \mathrm{~mJ}$ in $120 \mathrm{fs}$. The pulses are twice transform-limited and focusable to 1.5 the diffraction limit. Forty percent of the pulse energy is lost to a low irradiance halo around the focus. The second system is an amplified titanium-sapphire ( $\mathrm{Ti}: \mathrm{Al}_{2} \mathrm{O}_{3}$ ) laser operating at $800 \mathrm{~nm}$. The $\mathrm{Ti}: \mathrm{Al}_{2} \mathrm{O}_{3}$ laser system produces Gaussian pulses with energies of up to $60 \mathrm{~mJ}$ in 130 femtoseconds. The pulses are near transform-limited and focus cleanly to approximately 1.7 times the diffraction-limit. 


\section{CHAPTER 5 - EXPERIMENTAL CONFIGURATION}

\subsection{Introduction}

In these experiments I measure the number of ions produced in the focus of the laser versus irradiance. Figure 5.1.1 is a generic diagram for the experiments. The laser beam passes through a thin glass slide, and an energy meter ratiometer (Molectron JD2000 with a J3-09 power head and a J50 Power head) measures the $4 \%$ reflection from the slide. I align the beam to a pair of alignment irises spaced by $5 \mathrm{~m}$. The beam then passes through a $\lambda / 4$ waveplate that is rotated to produce the appropriate beam polarization. The beam then enters a vacuum chamber through a fused-quartz window. The base pressure of the vacuum chamber is $10^{-7} \mathrm{~Pa}$. The pumping system consists of a turbomolecular pump (Balzers TPU 520) backed by another turbomolecular pump (Balzers TPH 060) and a roughing pump. The target gases are introduced into the chamber as a constant flow through a sapphire valve. The gas enters a small premixing chamber attached to the main chamber, and then flows into the target chamber. A residual gas analyzer (Balzers QMG-064/QME-064) monitors the pressure of the target gas. The target pressures range from $10^{-3}$ to $10^{-6} \mathrm{~Pa}$.

An off-axis parabolic mirror focuses the laser pulses to produce the large irradiances needed for optical field ionization. The mirror (Space Optics Research Labs OAP 04-01.56-02) has a focal length of $10 \mathrm{~cm}$, an off-axis distance of 1.55 inches and a diameter of 1.999 inches. For the $614 \mathrm{~nm}$ experiments the mirror coating is a single-stack dielectric with a surface accuracy of $1 / 9$ wave peak to valley per inch over $93 \%$ of the coated surface. For the $800 \mathrm{~nm}$ experiments the mirror coating is silver. Aligning the off-axis parabola 


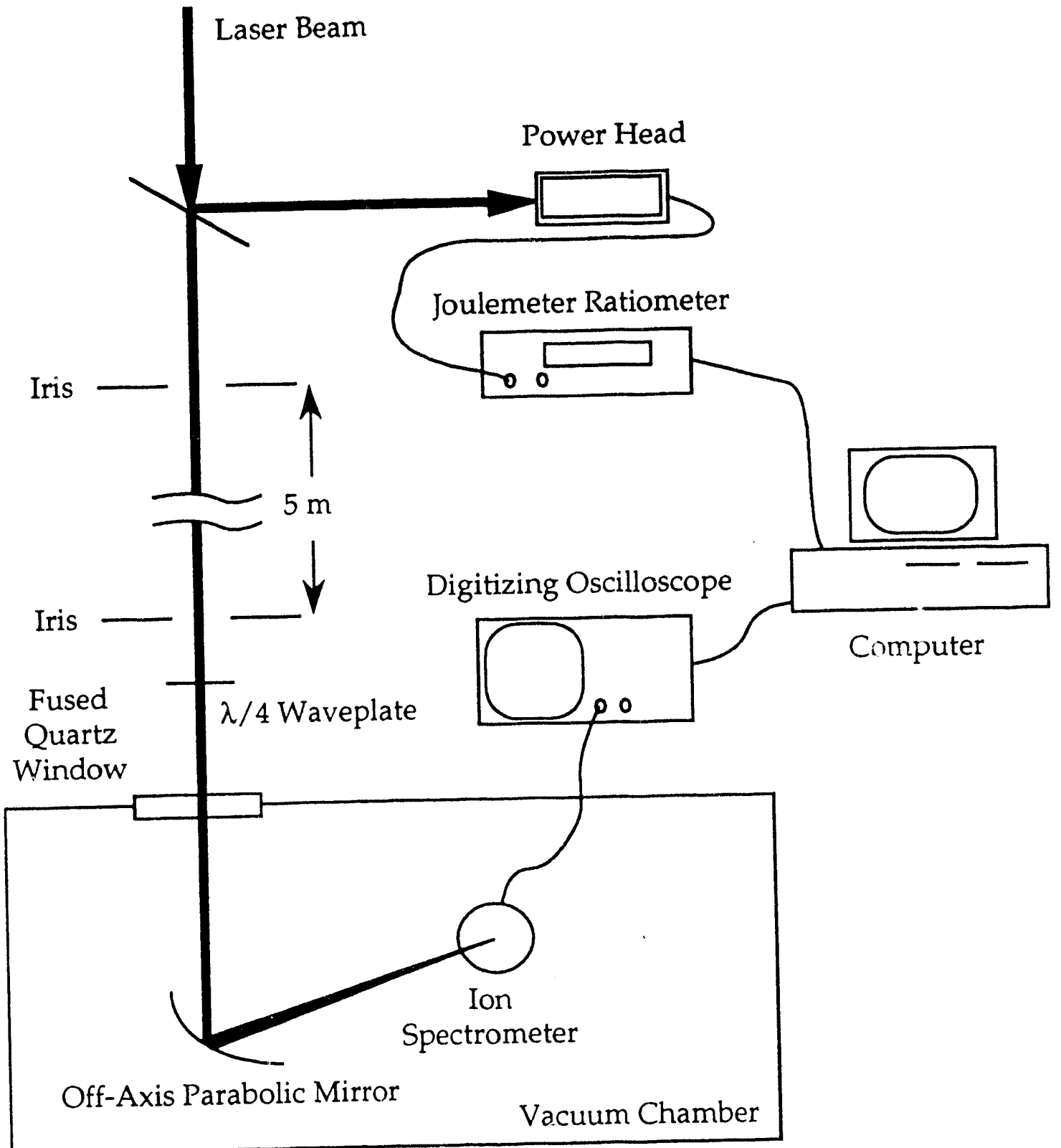

Figure 5.1.1 Diagram of the ion yield experiment.

requires controlling the input angle of beam to approximately $200 \mu \mathrm{rad}$. The alignment procedure is as follows. First, the beam is aligned through a pair of irises 5 meters apart and onto the parabola. A pellicle in the beam allows a camera to image the focal spot in reflection from a mirror placed at the focus. During the measurements the lasers operate at full power; a half-waveplate and 
polarizing beamsplitting cube placed before the irises reduce the pulse energy to below the damage threshold of the mirror at the focus. The beam reflected from the mirror is autocollimated back through the irises-that is the reflected beam must be the same size and shane as the incident beam. The camera image of the focal plane verifies that the resulting focal spot is symmetric and as small as possible. Leaving the imaging system in place for several operating days confirmed that the iris spacing was sufficient to regain the alignment each day. The resulting focal properties are discussed in Chapter 4 . The high irradiances at the focus then ionize the target gas. The mass spectrometer collects the ions produced in the focus. The spectrometer signal is recorded using a digitizing oscilloscope. A computer then records the laser energy and number of ions produced on each shot. 


\subsection{The Ion Spectrometer}

Figure 5.2.1 is a diagram of the linear mass spectrometer (Comstock TOF101 Time-of-Flight Mass Spectrometer) that collects the ions produced in the focus. The laser focuses between a plate and a grid that are separated by a distance $S$. For use in this experiment the plates and grids have been cropped to accommodate the converging beam path. The focus is a distance $s$ from the grid. The plate is held at a voltage $V_{1}$ and the grid at a voltage $V_{2}\left(V_{2}<V_{1}\right)$. This produces an electric field, $E_{S}$, that drives any ions produced through the grid and

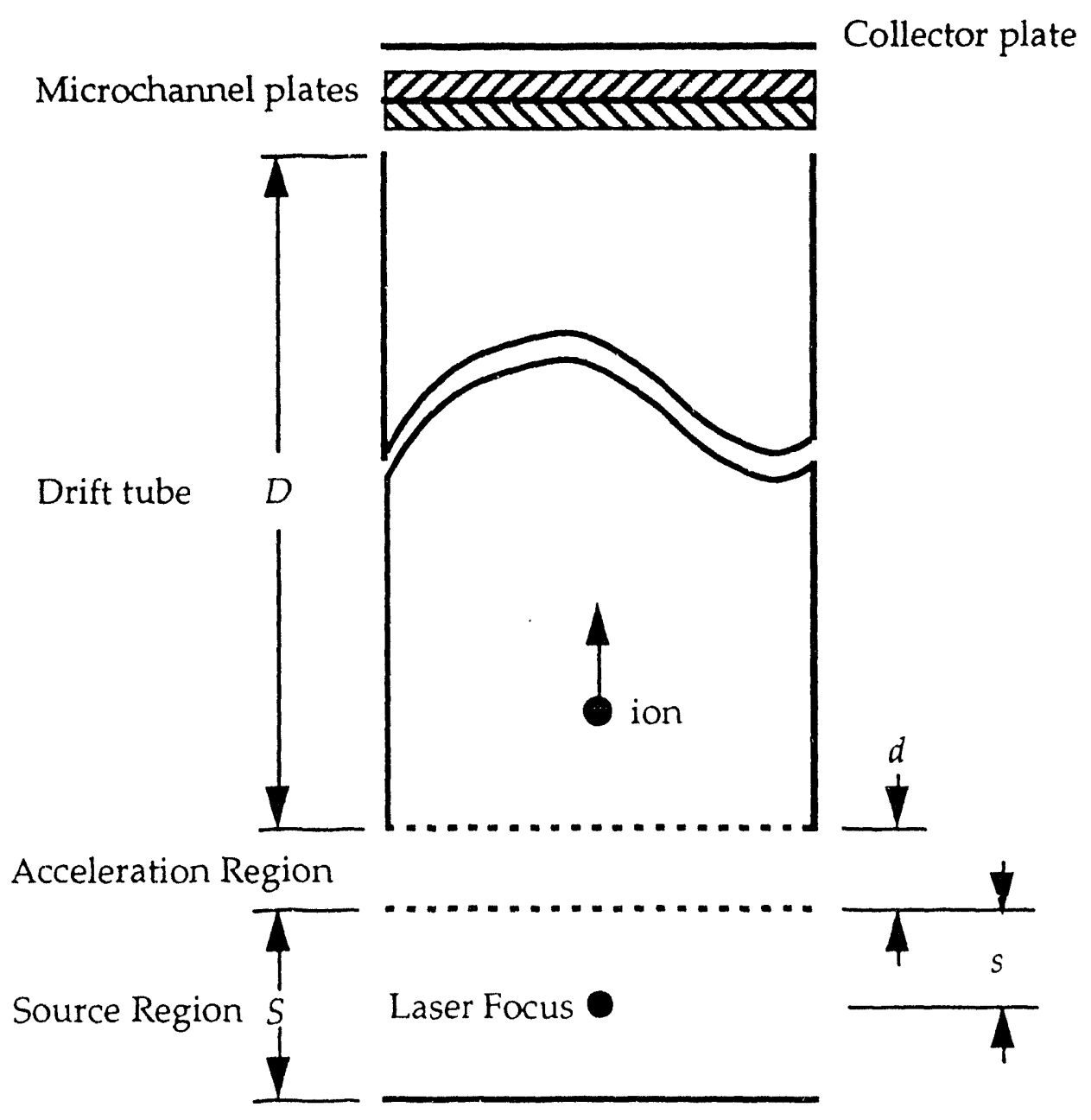

Figure 5.2.1 Ion Time-of-Flight Tube. 
into the acceleration region. The drift tube is held at a voltage $V_{3}\left(V_{3}<V_{2}\right)$. This produces an electric field, $E_{d}$, that accelerates the ion into the drift tube. To maintain a uniform field in the acceleration region and in the drift tube, wire grids cover the ends of the drift tube. Once in the drift tube, the ion experiences no electric field. Thus the ion drifts the length of the tube at the velocity at which it entered the tube. Upon reaching the end of the flight tube, the ion passes through another grid, across a small gap and strikes a pair of chevron-configured microchannel plates. The ion striking the plates causes a cascade of electrons that strike a collector plate. The gain of the channel plates is approximately $10^{6}$ and the charge striking the collector plate produces a measurable current that is proportional to the number of ions that strike the channel plate.

Let the electric fields be given in volts per centimeter. The total drift time in the spectrometer for an ion of mass $m$ (in amu), charge $q$ (units of electron charge) and initial energy $U_{0}$ (in $\mathrm{eV}$ ) is[56].

$$
T\left(U_{0}, s\right)=T_{s}+T_{d}+T_{D}
$$

where the time in the source region, $T_{S}$, is

$$
T_{s}=\frac{1.02(2 m)^{1 / 2}}{q E_{s}}\left[\left(U_{0}+q s E_{s}\right)^{1 / 2} \pm U_{0}^{1 / 2}\right] .
$$

the time in the acceleration region, $T_{d}$, is

$$
T_{d}=\frac{1.02(2 m)^{1 / 2}}{q E_{d}}\left[U^{4 / 2}-\left(U_{0}+q s E_{s}\right)^{4 / 2}\right],
$$

and the time in the drift tube, $T_{D}$, is

$$
T_{D}=1.02(2 m)^{1 / 2} D / 2 U^{1 / 2}
$$


Here

$$
U=U_{0}+q s E_{s}+q d E_{d}
$$

For ionization by ultra short laser pulses, $U_{0}$ is negligible for low-density gas targets at room temperature. Before the laser pulse arrives, the initial energies of the atoms are less than $.05 \mathrm{eV}$. Since the ions do not leave the laser focus during the pulse, they do not retain any pondermotive energy, and the only gain in energy is some of the excess photon energy or about one electron volt. Thus the total flight time of an ion to the microchannel plate detectors may be written in the form

$$
T(0, s)=C \sqrt{\frac{m}{q}}
$$

where $C$ is a constant and $T=0$ is the time that the laser pulse ionizes the atom. $C$ is of course calculable using Equations 5.2.1-5.2.6. For these experiments,

$$
\begin{array}{ll}
D=100 \mathrm{~cm}, & V_{1}=600 \mathrm{~V}, \\
d=.381 \mathrm{~cm}, & V_{2}=0 \mathrm{~V} \\
s_{\max }=.953 \mathrm{~cm}, & \text { and } \\
s=.477 \mathrm{~cm}, & V_{3}=-1622 \mathrm{~V} .
\end{array}
$$

I calculate that for these conditions, the total flight time should be

$$
T(\mu s)=1.7074 \sqrt{\frac{m}{q}} .
$$

Experimentally, I confirmed this prediction by measuring the flight time of known ions. Figure 5.2.2 shows the measured flight times versus the mass- 


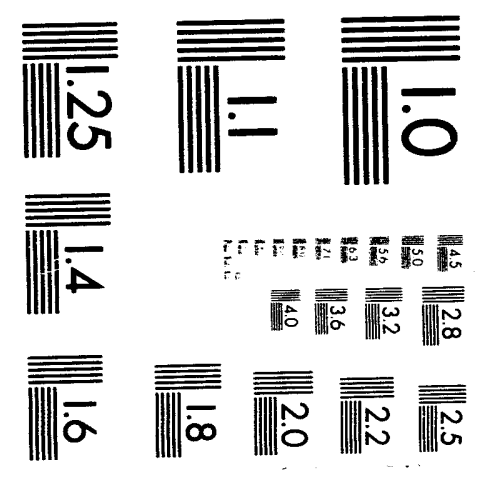



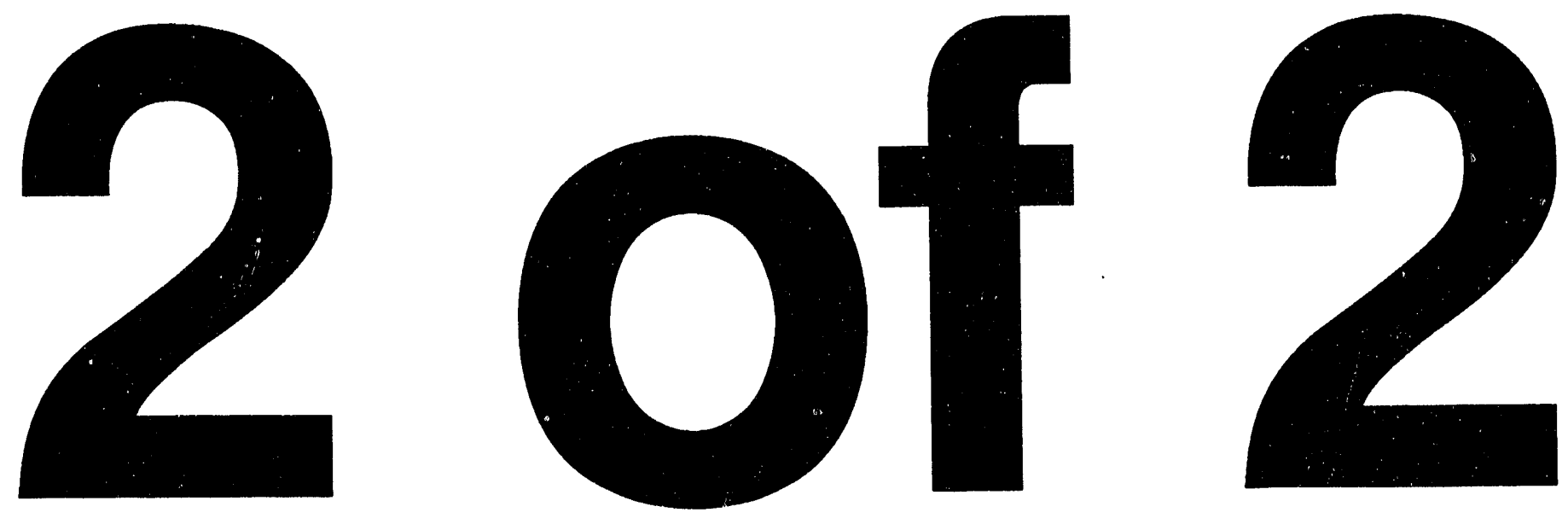


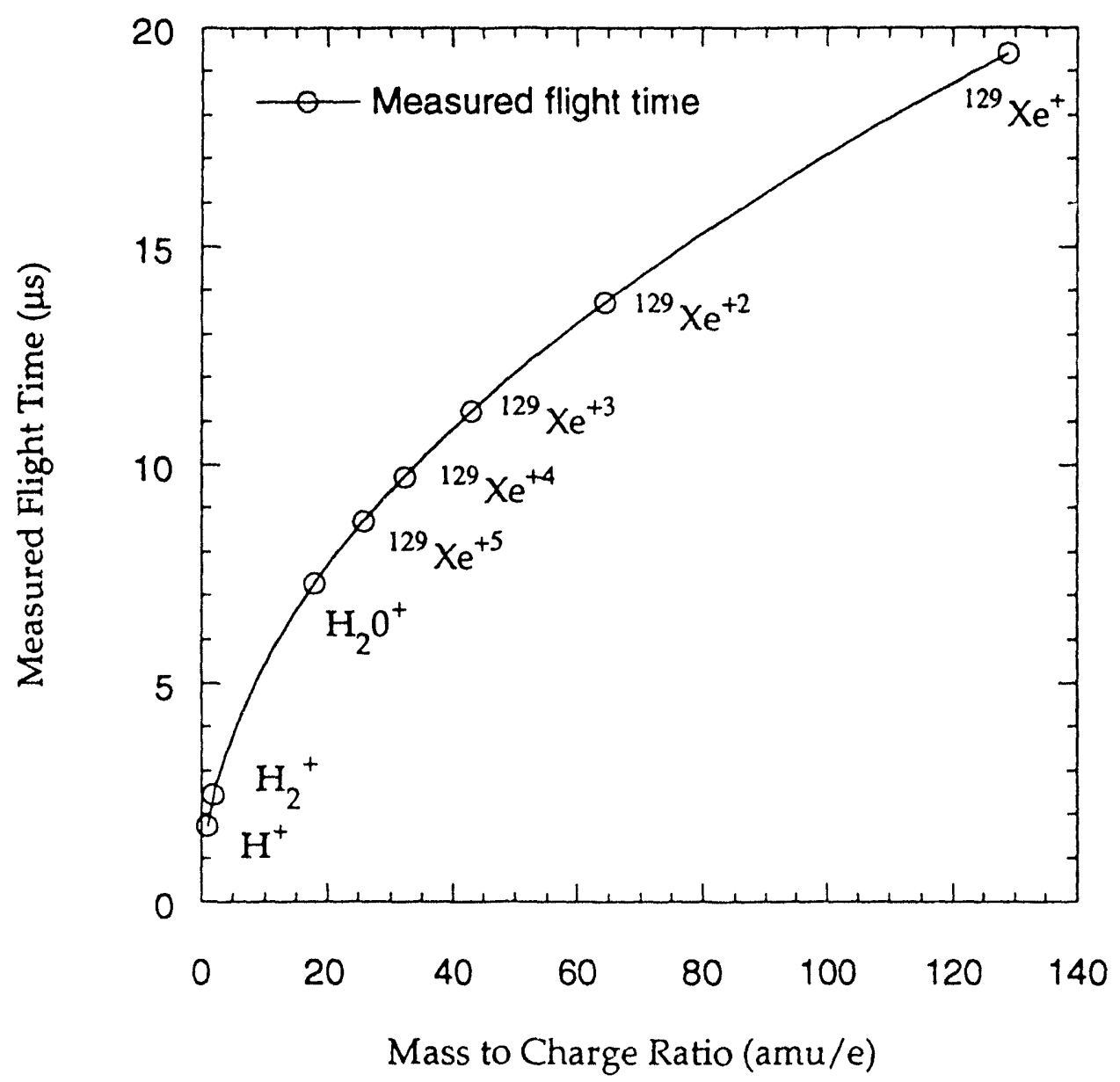

Figure 5.2.2 The measured flight time as a function of mass-tocharge for several ions. The solid line is a least-squares fit of the form $T(\mu s)=c_{1}+C \sqrt{m / q}$. The fitted parameters are $C=1.7069$ and $c_{1}$ $=.025 \mu \mathrm{s}$. The $\chi^{2}$ for the fit is $4.894 \times 10^{-5}$, and the probability that the data set could have come from the theory is essentially unity. The maximum error for any single measurement was $3 \mathrm{~ns}$. 
to-charge ratio of the ions. The least-squares fit gives an intercept $c_{1}$ that defines $\mathrm{T}=0$. This is very useful since accurately measuring all the timing delays between the arrival of the pulse at the focus, the arrival of the ion at the channel plates, the arrival of the signal at the oscilloscope and the trigger for the oscilloscope is difficult. The fitted value of $C$ is 1.7069 and is only $.03 \%$ different from the prediction of Equation (5.2.7). Also the maximum error between the fit and any single measurement was 3 ns. This is important because it allows accurate determination of unknown species of similar mass-to charge ratio.

Due to its length, the spectrometer can even discern mass-to-charge ratio differences as small a one part in two hundred. Because the mass of $\mathrm{H}_{2}$ is $2.0156^{\circ}$ amu and the mass of ${ }^{4} \mathrm{He}$ is $4.0026, \mathrm{H}_{2}{ }^{+}$is a potential contaminant in $\mathrm{He}^{+2}$ ion yield measurements. Figure 5.2.3 shows the $\mathrm{He}^{+2}$ and $\mathrm{H}_{2}{ }^{+}$ion signals as a function of time. As is apparent in the figure, the two signals are separable, and the $\mathrm{He}^{+2}$ yield measurements are free of contamination.

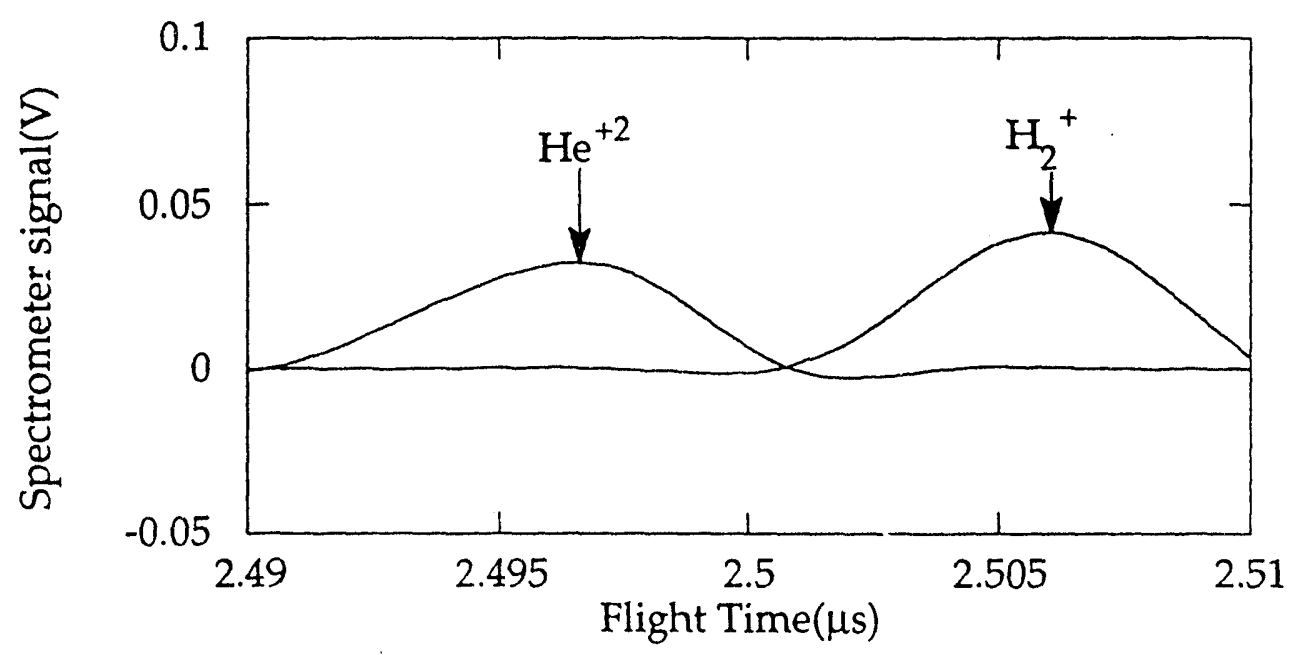

Figure 5.2.3 The spectrometer resolution is shown clearly by the separation of the $\mathrm{He}^{+2}$ and $\mathrm{H}_{2}{ }^{+}$signals. 
Space-charge[57] can alter the flight time of the ions when the laser pulse produces tens of thousands of ions. For ionization by $120 \mathrm{fs}$ pulses, the ions and electrons do not move appreciably during the pulse. After the pulse passes, however, the electric field in the source region of the spectrometer separates the electrons and ions. Also the relativeiy fast electrons move away from the focal region more rapidly than the ions. Without the electrons in the volume, the Coulomb interaction between the ions can lead to the ions having large potential energy. As one expects upon study of Equations 5.2.1-5.2.5 for large $U_{0}$, this interaction can greatly alter the observed ion spectrum. Figure 5.2.4 shows the ion signals for ${ }^{20} \mathrm{Ne}^{+1}$ as a function of time for several different pressures. The laser irradiance was approximately $2 \times 10^{15} \mathrm{~W} / \mathrm{cm}^{2}$ for linearly polarized $800-\mathrm{nm}$ pulses. As the pressure increases, the ion signal broadens due to the Coulomb interaction between the increasing number of ions. The spread of the flight times indicates that the energy due to Coulomb repulsion can be on the order of $60 \mathrm{eV}$ for a pressure of $1 \times 10^{-3} \mathrm{~Pa}$. Based on the number of ions produced (approximately 30,000 is the ADK prediction), the energy for a particle-due to interactions with its neighbors-at the $1 / \mathrm{e}^{2}$ radius of the beam $(8.1 \mu \mathrm{m})$ should be $5 \mathrm{eV}$. This discrepancy may be due to focal volume effects. The irradiance for the mcasurement is more than 10 times the appearance irradiance for $\mathrm{Ne}^{+1}$, and any low irradiance spatial structure will begin to contribute to the number of ions produced. To avoid such space-charge effects during the experiments the pressure was varied to allow production of only a few ions per shot.

In Figure 5.2.4 the actual number of ions detected does not increase linearly with the pressure of the target gas. At the lower pressures the Coulomb effects are negligible, and all the ions that enter the drift tube reach the microchannel plates. At higher pressures the Coulomb effects impart a large transverse velocity 


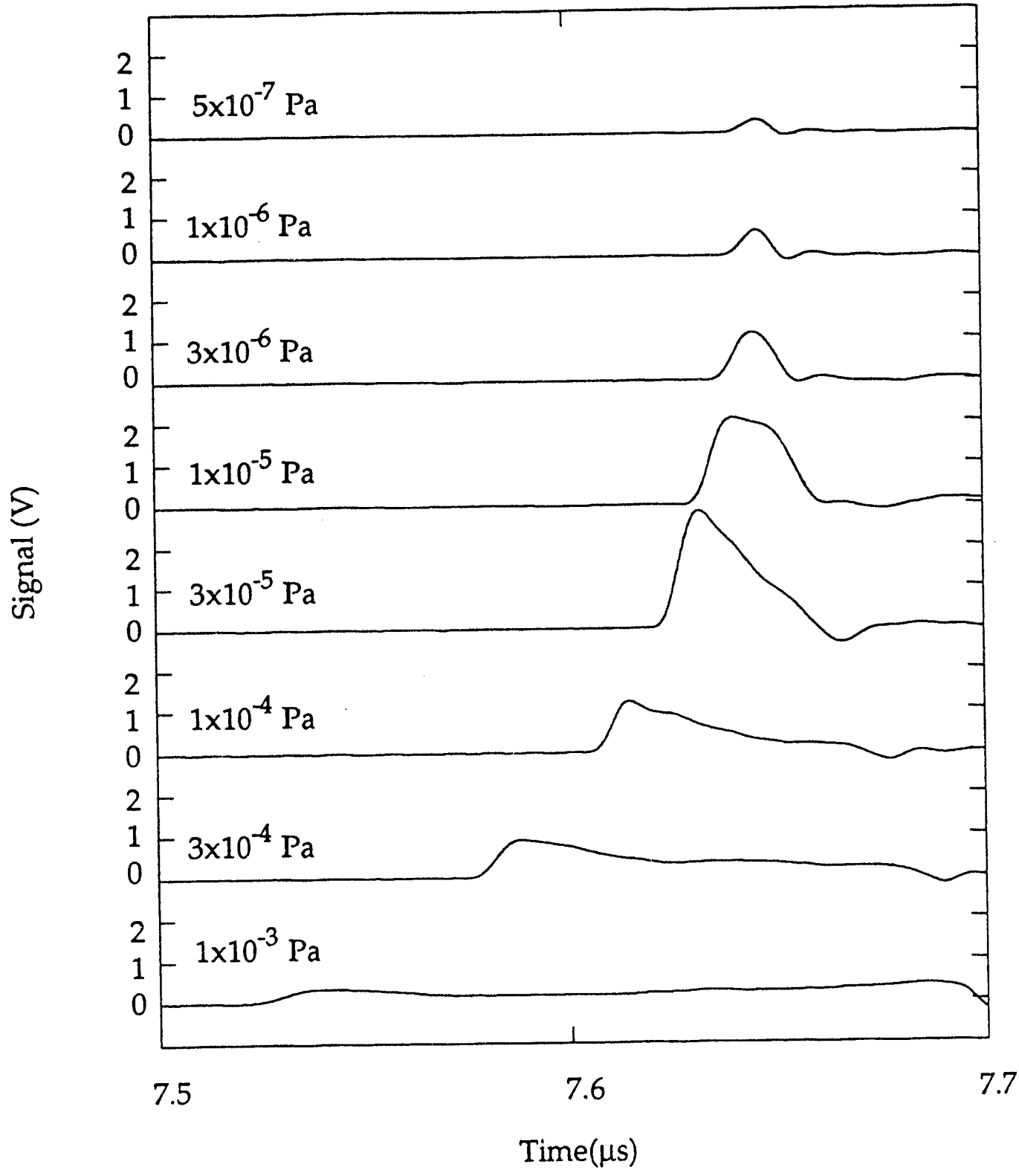

Figure 5.2.4 The ion signal as a function of time for several different pressures. The charge state is $20 \mathrm{Ne}^{+1}$. The laser irradiance was approximately $2 \times 10^{15} \mathrm{~W} / \mathrm{cm}^{2}$ for linearly polarized $800-\mathrm{nm}$ pulses. As the pressure increases the ion signal broadens due to the Coulomb interaction between the increasing number of ions. The integrated number of detected ions increases and then decreases. 
to the ions forcing them into the walls of the drift tube. This decreases the effective solid angle of the flight tube. Also saturation of the microchannel plates can decrease the detection efficiency. As long as the number of ions created remains below a few thousand, the number of ions detected is linear in the pressure of the target gas as shown in Figure 5.2.5 for $\mathrm{Ne}^{+1}$. This is important because it allows variations of the gas pressure in order to maintain a constant detection efficiency at all irradiances.

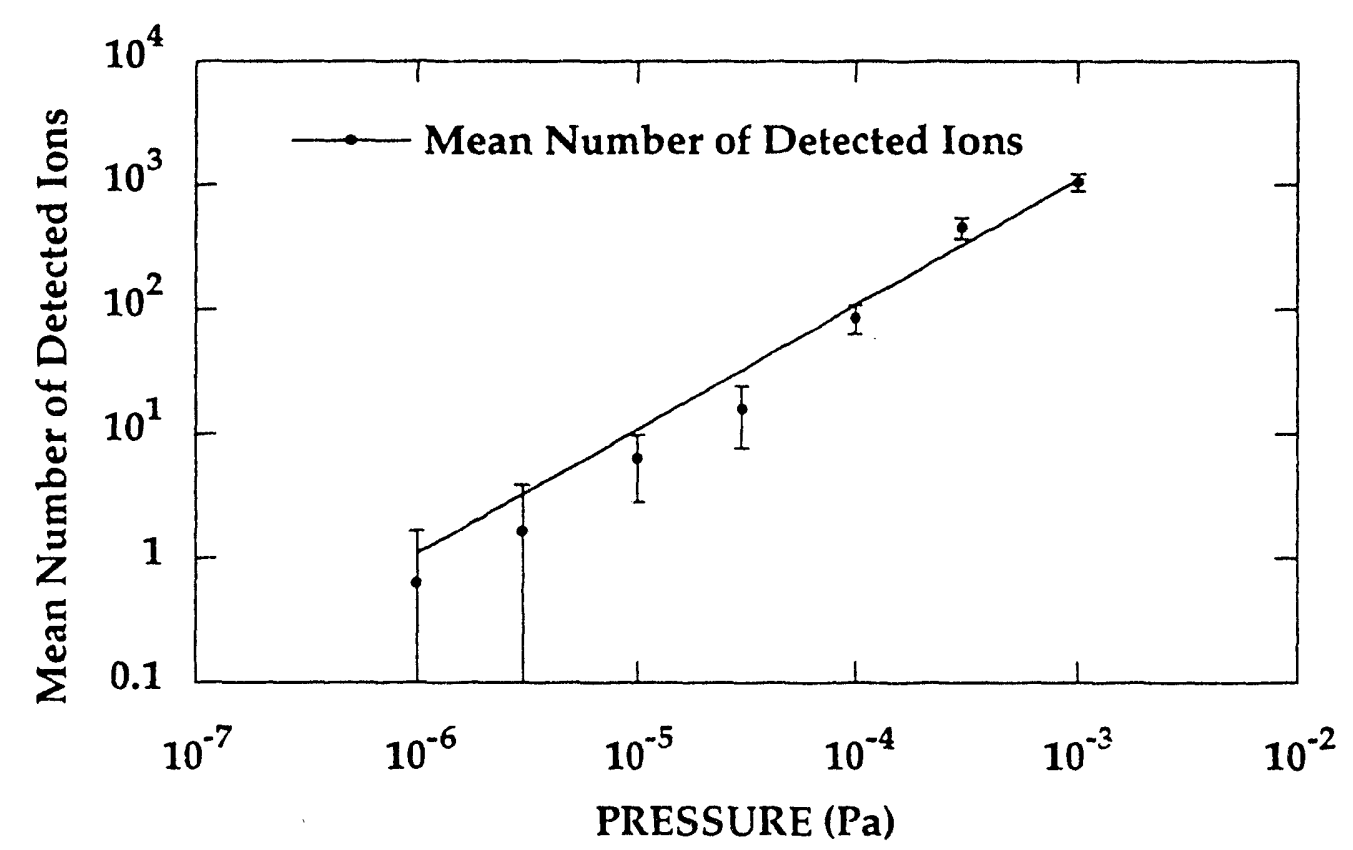

Figure 5.2.5 The pressure dependence of the ion yields using the $800 \mathrm{~nm}$ laser. The $\mathrm{Ne}^{+1}$ yields are averaged over fifty shots at energies between .2211 and $.2214 \mathrm{~mJ}$. The solid line indicates a linear pressure dependence. The error bars indicate one standard deviation in the number of ions detected. For fewer than 1000 ions, the ion yields scale with pressure. 


\subsection{The Microchannel Plate Detector}

The actual detection of the ions occurs at the $40 \mathrm{~mm}$ diameter microchannel plates located at the end of the spectrometer as shown in Figure 5.2.1. A microchannel plate is essentially a thin glass plate containing a large number of dynode ion multipliers set closely together[58-63]. The channels in the plates are set at an $8^{\circ}$ angle to the normal axis of the plates. The two plates are mounted with their channels oriented in a chevron configuration to produce the maximum angle between the channels. This configuration provides the minimum ion feedback and provides high gains[62].

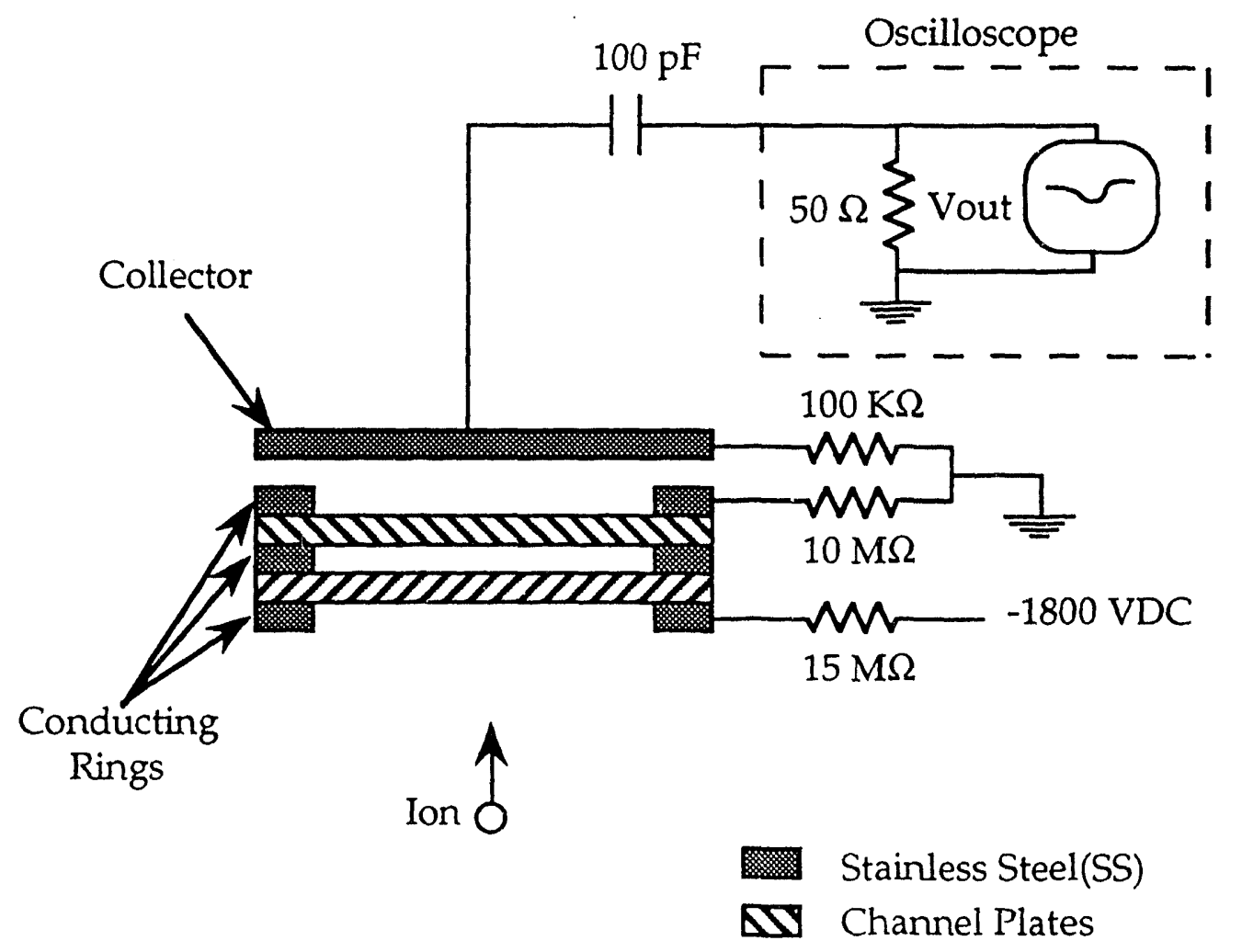

Figure 5.3.1 Schematic of the ion detector. The current detected at the collector plate is capacitively coupled into a $50 \Omega$ oscilloscope. 
Figure 5.3.1 is a schematic of the ion detector. The voltage across each channel plate is approximately $900 \mathrm{~V}$. The current detected at the collector plate is capacitively coupled into a $50 \Omega$ oscilloscope. The actual mean signal due to a single ion was obtrined by pulse height analysis of several thousand data shots. The pulse irradiance and pressure were low enough so that most shots recorded no signal. Thus the majority of the shots gave zero integrated signal, and at most a few ions were detected on any given shot. Figure 5.3.2 shows the results of the pulse height analysis. The regular series of occurrences shows that the integrated

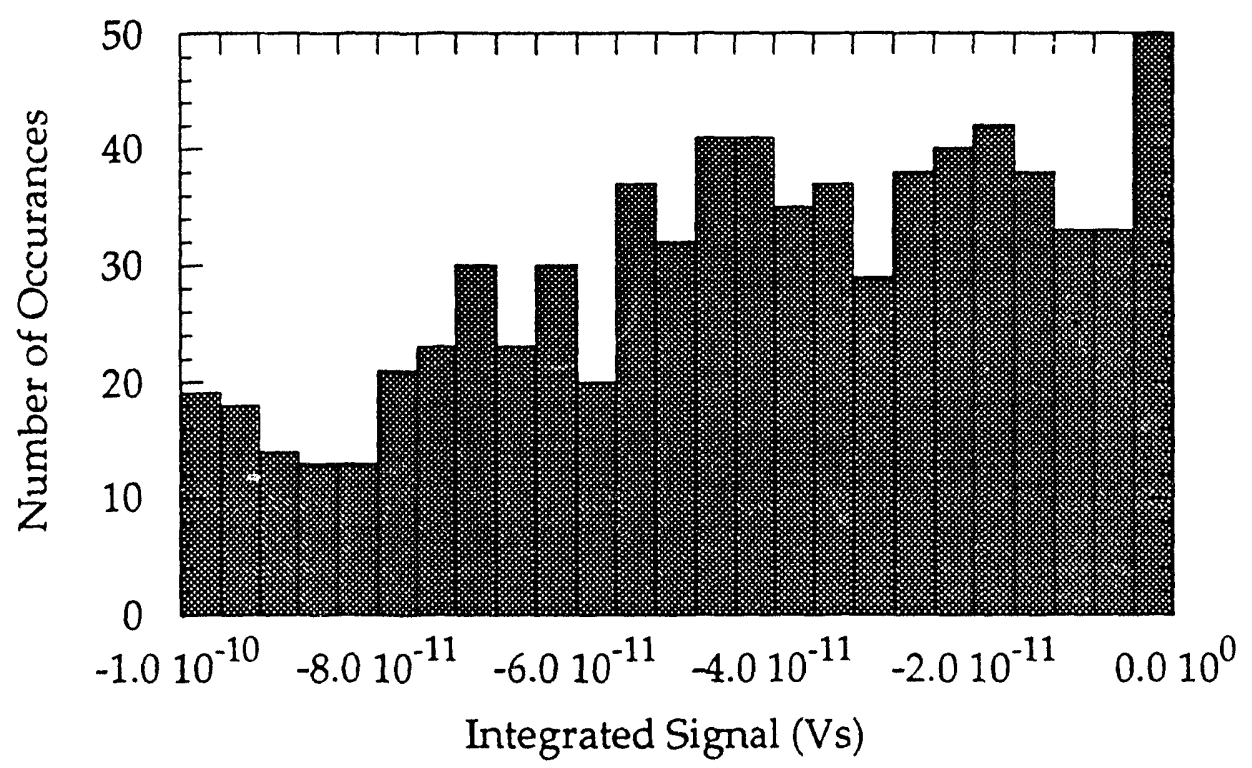

Figure 5.3.2 Results of the pulse height analysis of several thousand data shots.

single-ion signal is approximately $-2.2 \times 10^{-11} \mathrm{~V}$-sec. The single-ion signal has a pulse width of approximately $5 \mathrm{~ns}$. Thus the detector has a gain of 2-3 million that is consistent with the gains for tandem channel plates[62]. Dividing the integrated signal by this single-ion signal gives the number of ions detected. 
The overall efficiency (number of ions detected per ion produced) of the flight tube is calculated using the transparency of the grids (90\%) and the quantum efficiency (number of signals per ion impinging on the microchannel plates) of the microchannel plate detectors (at $\approx 35 \%$ [64] this represents the largest uncertainty in the ion counting - a factor of a few) The efficiency increases by a factor of approximately 1.25 for the doubly ionized charge states due to their increased energy.

It is important in counting the doubly charged ions that the doubly charged ions arrive before singly charged ions. The production of the doubly charged ions typically requires the production of thousands of singly charged ions. This total number of ions can saturate the microchannel plates, The doubly charged ions do, however, arrive before saturation occurs, and those are counted correctly as long as space-charge effects are negligible.

\subsection{Data Acquisition}

The data acquisition computer is an Apple Macintosh Ifx with a National Instruments NB-DMA-02800 GPIB board. A program written in LabView drives the oscilloscope and the power meter. For both laser systems, the acquisition system triggers off a diode placed to detect the zeroth-order beam off the first grating in the compressor. The program reads the trace from the oscilloscope and integrates it over the time range set by the operator. As noted previously, the time windows were set to include only the charge state of interest. Typical widths were $10 \mathrm{~ns}$ for $\mathrm{He}^{+2}$. The program also reads the power meter to obtain the energy in the pulse reflected off the beam splitter, and applies a calibration factor to obtain the energy on target. The computer the writes the integrated signal and energy a file. After the data are taken, other programs bin the shots by 
energy, average the shots within the bins and convert the integrated signal to number of ions produced. The pressures used in the runs vary from $10^{-3} \mathrm{~Pa}$ to $10^{-}$ $6 \mathrm{~Pa}$. All data shown in this work are normalized to a pressure of $10^{-3} \mathrm{~Pa}$. One program also calculates the peak irradiance from the pulse energy.

\subsection{Summary}

This experiment counts the number of ions produced in the focus of the lasers versus peak irradiance at the focus. The high resolution of the mass spectrometer allows accurate determination of the ion species and even eliminates contamination of the $\mathrm{He}^{+2}$ signal by $\mathrm{H}_{2}{ }^{+}$. The spectrometer efficiency and signal for a single event are known, and this allows the actual number of ions produced in the laser focus to be determined. Careful selection of the target gas pressure allows production of only a few ions per shot and avoids microchannel plate saturation and space charge effects. 


\section{CHAPTER 6 - 614NM IONIZATION DATA AND NONSEQUENTIAL IONIZATION}

\subsection{Introduction}

In this chapter I present the measured ion yields for singly and doubly charged ions of $\mathrm{He}$ and $\mathrm{Ne}$ created by linearly polarized laser pulses at $614 \mathrm{~nm}$. The peak irradiances exceed $10^{16} \mathrm{~W} / \mathrm{cm}^{2}$. I compare the data for linear polarization to sequential tunneling theory. To explain the discrepancies between the data and the predictions of sequential tunneling theory, two possible mechanisms for nonsequential ionization are discussed. The first mechanism is a sudden approximation where the first ionization occurs so rapidly that the inner electron cannot readjust to the new ionic potential and is removed[17]. The second mechanism is a rescattering mechanism where the laser field drives the first electron removed back toward the ion core. The electron then scatters from the core causing a second ionization[65]. The two nonsequential models make significantly different predictions about the polarization and wavelength dependence of the ionization, and I will present data for circular polarization to discern which model is more likely to be correct.

\subsection{Linear Polarization Data at $614 \mathrm{~nm}$}

Figure 6.2.1 shows the measured singly and doubly ionized $\mathrm{He}$ and $\mathrm{Ne}$ ion yields versus peak irradiance for linearly polarized 614-nm pulses. As noted previously the pulses have a squared hyperbolic-secant temporal profile with a FWHM of $120 \mathrm{fs}$. The focal spot has a FWHM of $4.5 \mu \mathrm{m}$. All data plotted in this dissertation are scaled to a target pressure of $1 \times 10^{-3} \mathrm{~Pa}$ unless otherwise noted. The data are unshifted with respect to the measured irradiance or with respect to each other. The data for each charge state represents 9,000 to 11,000 laser shots binned and 


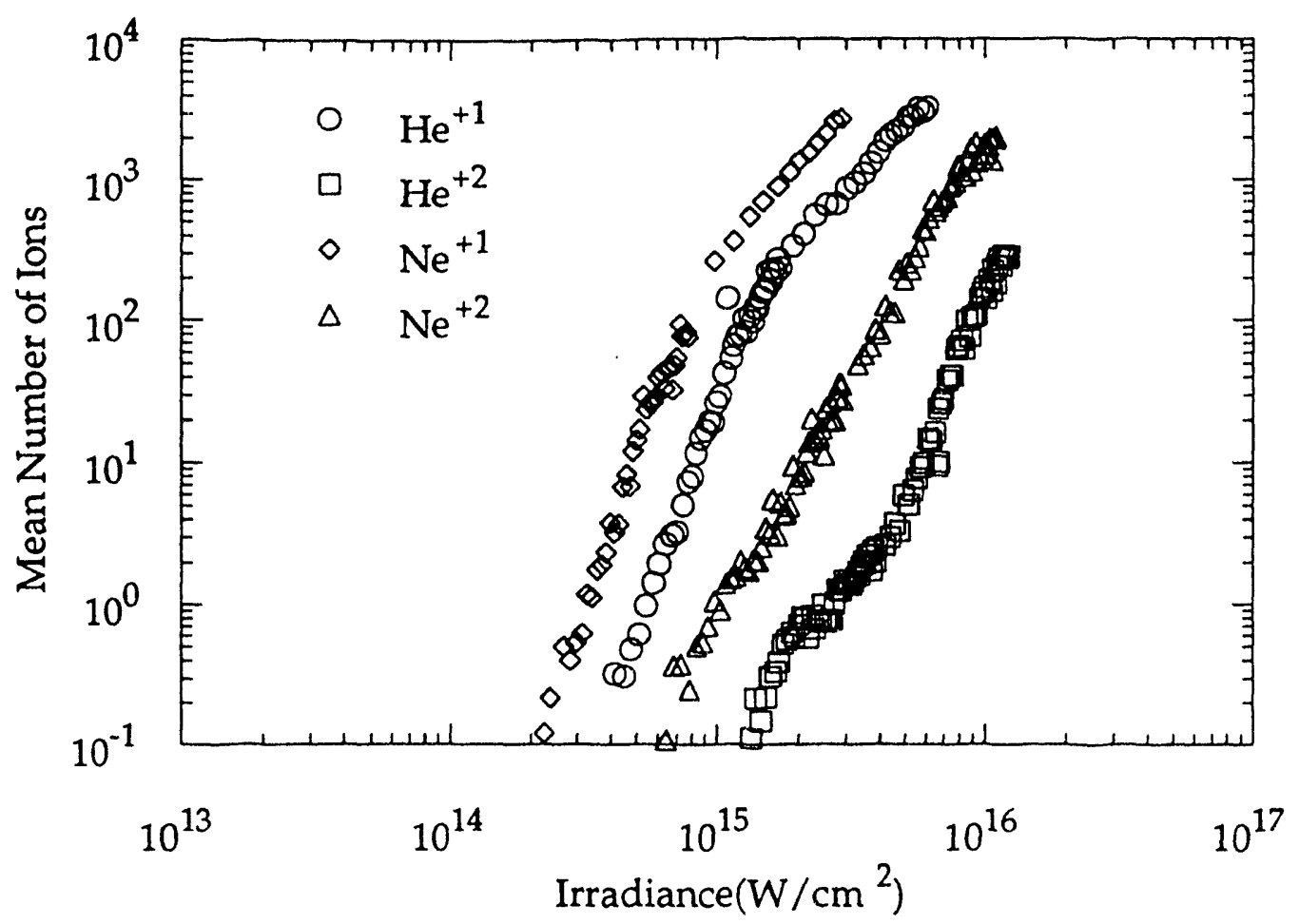

Figure 6.2.1 Helium and neon ion yields for 120-fs linearly polarized pulses at $614 \mathrm{~nm}$.

averaged in approximately 100 evenly spaced bins. Measurements have been repeated several times and show no discernible shift in the relative positions. Even before comparing the data directly with a specific ionization model the data is obviously unusual. As noted in Chapter 3, sequential tunneling should produce characteristic curves for the ion yields regardless of the charge state under consideration. The characteristic curves are largely functions of the ionization potential; therefore, the curves should not cross and always have a negative second derivative (i.e. the yields exhibit no inflection points). For $\mathrm{He}^{+1}$ and $\mathrm{Ne}^{+1}$ data, the ion yields rise smoothly with irradiance and then begin to volume saturate. For $\mathrm{He}^{+2}$ and $\mathrm{Ne}^{+2}$, however, the ion yields rise, saturate and then rise and begin to saturate again. As noted in Chapter 4 the high resolution of 
the time-of-flight spectrometer eliminates the possibility that the observed enhancements near the threshold irradiances are produced through contamination of other ions such as $\mathrm{H}_{2}{ }^{+}$.

Figure 6.2.2 confirms that the $\mathrm{He}^{+2}$ yield does not behave as predicted for sequential ionization. In the figure the data agree well with the SAE model for $\mathrm{He}^{+1}$ to within the uncertainty in the peak irradiance. For $\mathrm{He}^{+2}$, however, the yield rises early and saturates and then rejoins the curve predicted by the

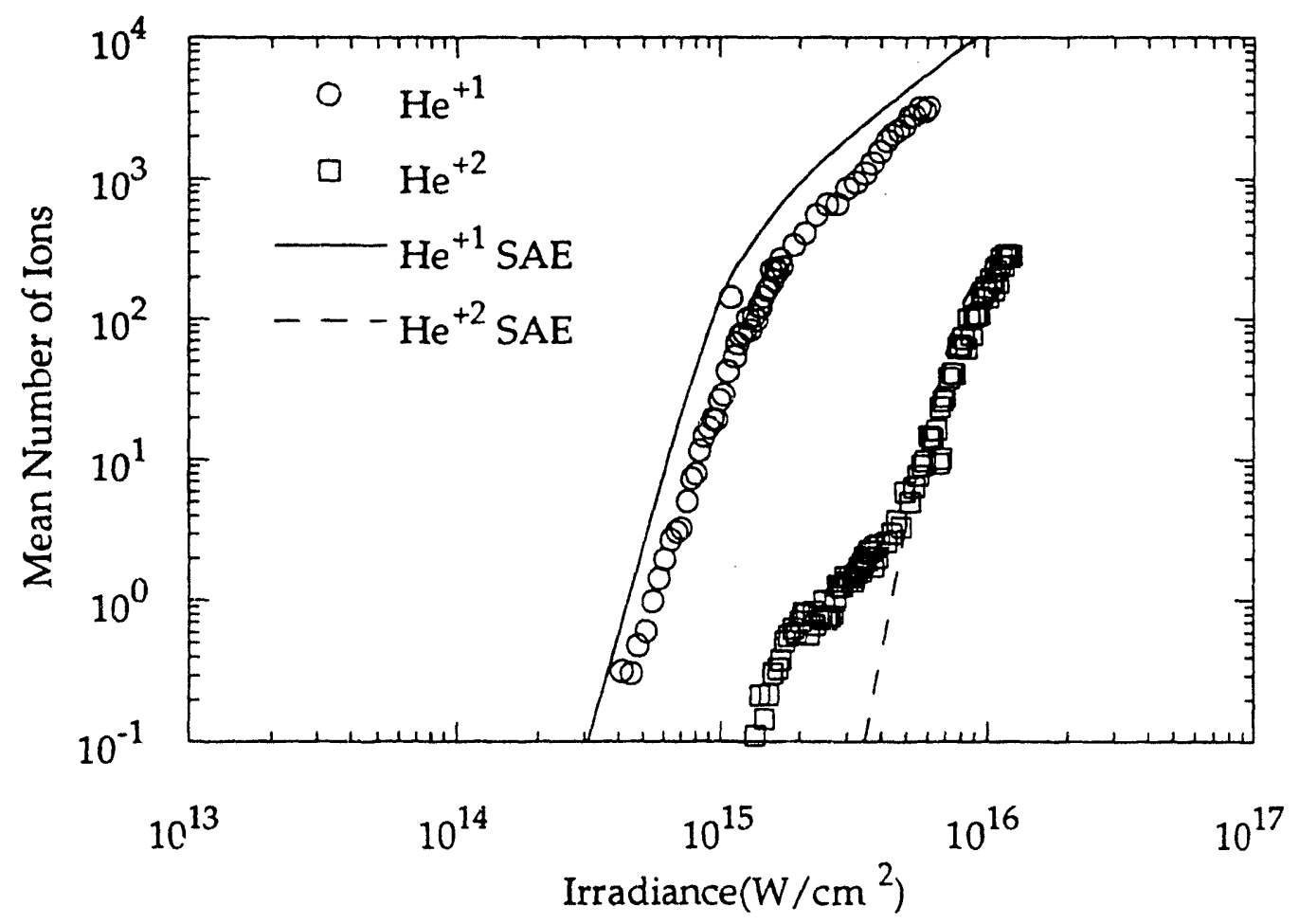

Figure 6.2.2 The helium ion yields superimposed on the yields predicted by SAE theory. The agreement for the singly ionized charge state is excellent within the experimental uncertainty. A large enhancement over SAE is apparent below $3 \times 10^{15} \mathrm{~W} / \mathrm{cm}^{2}$ for $\mathrm{He}^{+2}$. The ADK saturation irradiance from Table 3.7 .6 is $9.34 \times 10^{15}$ $\mathrm{W} / \mathrm{cm}^{2}$. (SAE calculations courtesy of Ken Kulander[17].) 
sequential SAE model. Note also this unexpected feature in the yield begins to volume saturate at the same irradiance as the $\mathrm{He}^{+1}$ yield.

The SAE model, while the best available solution of the Schrödinger equation for ionization, is not currently calculable for neon or other multi-electron atoms or for polarizations other than linear. Thus I will compare the data for neon and helium to predictions of the yields using the ADK model. The agreement between the ADK and SAE models is already shown in Figure 2.4.1. Figure 6.2.3 shows the ion yields superimposed on the predictions of sequential ADK

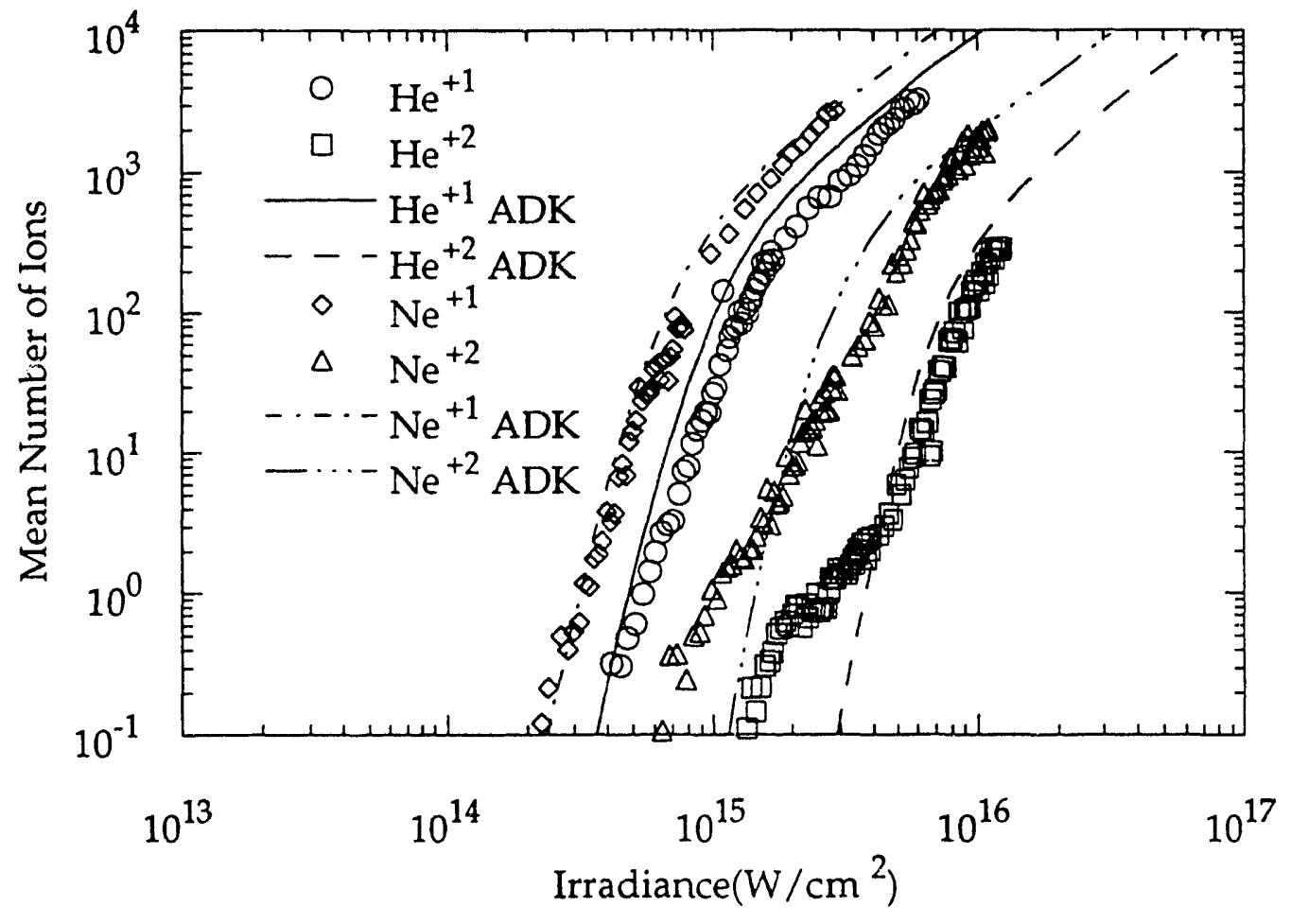

Figure 6.2.3 The singly and doubly charged helium and neon ion yields superimposed on the predictions for sequential ADK theory.

theory[27, 31]. As expected, the figure shows excellent agreement of the singly ionized charge states with the ADK model predictions to within the uncertainty 
in the irradiance. As in the case of helium, the $\mathrm{Ne}^{+2}$ yield appears at an irradiance lower than the ADK appearance irradiance. The yield then rises and saturates at the same irradiance as $\mathrm{Ne}^{+1}$. Eventually, again as for helium, the yield rises to follow the sequential ADK prediction for the $\mathrm{Ne}^{+2}$ yield. As noted in Chapter 3, the ADK appearance irradiances for the singly charged ions of each should exhibit a cubic dependence in the ionization potential[41]. The observed ratio of the $\mathrm{He}^{+1}$ and $\mathrm{Ne}^{+1}$ appearance irradiances is $3 / 2$ and agrees with this closed-form prediction. The cubic dependence is discussed further in Chapter 8.

\subsection{Resonance Effects}

Resonantly enhanced ionization rates do not explain our observations. In resonantly-enhanced ionization, the ionization rate increases when the energy of an allowed atomic transition nearly equals the energy of an integral number of photons. Changing to circular polarization would significantly decrease any resonance effects-in agreement with the experiments-because of the decreased number of allowed electronic transitions. Since dynamic resonances can occur as the atomic energy levels shift in response to the applied field, resonances do become likely in terms of energy levels being equal to an integer number of photons at some point during the pulse. In addition, experiments in the multiphoton regime show similarly enhanced xenon ion yields for longer pulse widths $[15,16]$. Resonances, however, are unlikely to influence the results of tunneling experiments that are non-perturbative. As shown in Figure 6.2.2 direct solution of the Schrödinger equation for a single active electron (SAE) for helium shows no evidence of resonant enhancements[17]. The SAE calculation would miss two-electron effects. Enhancements due to double excitations are unlikely on energetic grounds. The double excited states of helium are $60 \mathrm{eV}$ above the ground state of the atom-almost $40 \mathrm{eV}$ above the first ionization potential. Also, 
the dynamics of the shifting of energy levels that produces dynamic resonance conditions suggest that these forced resonances are very short lived (on the order of three cycles on the rise and fall of the laser pulse) and are probably worth consideration only near the peak field amplitudes and envelope maximum. Walker et al. showed using electron coincidence experiments that direct ionization is not responsible for enhanced double ionization in Xe which occurs in the multiphoton regime[66]. Electron spectra for helium, however, clearly indicate that the ionization is nonperturbative $[67,68]$. The spectra agree with the ADK model predictions for tunneling. The perturbation theory scheme for ionization involving resonant states becomes physically unreasonable while direct models become more reasonable. For these reasons double ejection in the tunneling regime is unlikely to be due to resonance states.

\subsection{Nonsequential Ionization}

Since sequential ionization does not explain the data, nonsequential ionization-ionization including mechanisms directly connecting the neutral and doubly charged ion-must be considered. The rate equations (2.5.2) are one way to describe ionization generally. Again these are

$$
\begin{aligned}
& \frac{d p_{0}}{d t}=-\Gamma_{0} p_{0} \\
& \frac{d p_{1}}{d t}=-\Gamma_{1} p_{1}+\Gamma_{0}^{\prime} p_{0} \\
& \frac{d p_{2}}{d t}=\left(\Gamma_{0}-\Gamma_{0}^{\prime}\right) p_{0}+\Gamma_{1} p_{1}
\end{aligned}
$$

Here $p_{\mathrm{j}}$ and $\Gamma_{\mathrm{j}}$ are the probability of a being in a given charge state and the irradiance dependent total ionization rate for states of charge $j$, respectively. If the ionization process is purely sequential, $\Gamma_{0}{ }^{\prime}$ exactly equals $\Gamma_{0}$. If there is any process that transfers population directly from the neutral to the doubly charged 
ion, then $\Gamma_{0}^{\prime}<\Gamma_{0}$. Examination of the rate equations produces two general conclusions. First, the appearance irradiance for the second ion will be lower than that predicted by a purely sequential model. Second, any direct component will volume saturate in parallel with the single ion signal as the laser irradiance increases. As shown in Figure 6.2.3, the data agree with these conclusions. Details of the ionization processes determine the values of the irradiance dependent ionization rate coefficients. The physics of the nonperturbative direct nonsequential component would determine the scaling of observed ion yield enhancements with gas species, laser wavelength, ellipticity and pulse width.

A direct ionization mechanism[17] has been proposed to explain the data as similar to the shake-off process observed in single-photon double ionization of helium[69]. In this model the first electron absorbs a photon with enough energy to remove both electrons and rapidly leaves the vicinity of the nucleus causing the second electron to be "shaken up" into the continuum some fraction of the time. This occurs because the process is so rapid that the remaining second electron is in an excited state relative to the resulting ion. For photon energies from 80 to $120 \mathrm{eV}$, the shake-up probability is a few percent of the single ionization probability. For the multiple-photon case and photon energies of $2 \mathrm{eV}$, it is postulated that this process might also occur in the tunneling regime if the rate at which the first electron leaves the atom becomes large enough. The direct component would be measurable only if the pulse risetime is short enough since neutral population must still exist within the focal volume. The process would then exhibit an irradiance and rate threshold in single ionization such that above some critical irradiance, $I_{\mathcal{C}}$, and rate, $R_{\mathcal{C}}$, the process becomes possible. In other words $\frac{\Gamma_{0}^{\prime}}{\Gamma_{0}}<1$ for $I<I_{c}$ and $R>R_{c}$. Shake-off of the second electron would occur within much less than a quarter of an optical cycle. Using these assumptions 
produces a fit to the observed double ionization yields. In fitting the helium data, the probability is comparable to that in the single photon case, approximately half of one percent, and $I_{C}$ equal to $10^{15} \mathrm{~W} / \mathrm{cm}^{2}$ for helium. The corresponding critical rate is $R_{C}=1.2 \times 10^{14} \mathrm{sec}^{-1}$. The shake-off process should be strongly dependent on the pulse width of the laser and relatively independent of its wavelength and ellipticity.

Figure 6.4.1 compares the data for linearly polarized 614-nm pulses with a shakeoff prediction(DIR) for helium and neon. The prediction uses the linear ADK rates and evaluates the direct component using $\frac{\Gamma_{0}^{\prime}}{\Gamma_{0}}=0.995$ when $\Gamma_{0} \geq R_{c}$ and one otherwise. For neon the critical instantaneous rate (irradiance) for the onset of a direct ionization component is $4 \times 10^{13} \mathrm{sec}^{-1}\left(5 \times 10^{14} \mathrm{~W} / \mathrm{cm}^{2}\right)$

In contrast, Corkum et al. have proposed an alternative model for direct, nonsequential ionization that predicts a strong laser ellipticity and wavelength dependence $[65,70]$ This has in part motivated the examination of the ion yields with 800-nm light that is presented in Chapter 7. The model is presented in a unified way as also describing harmonic generation yields and ATI spectra. Regarding direct ionization it is a three-step quasiclassical process. First, the field of the laser suppresses the Coulomb barrier and liberates an electron by optical tunneling. Second, the liberated electron interacts with the laser field continuum. This produces an approximately $50 \%$ probability of accelerating the electron back toward the parent ion within a minimum time interval of one-half period. Here the laser and Coulomb fields are additive. Third, this electron removes a second electron from the ion by inelastic, e-2e scattering. The scattering probability is 


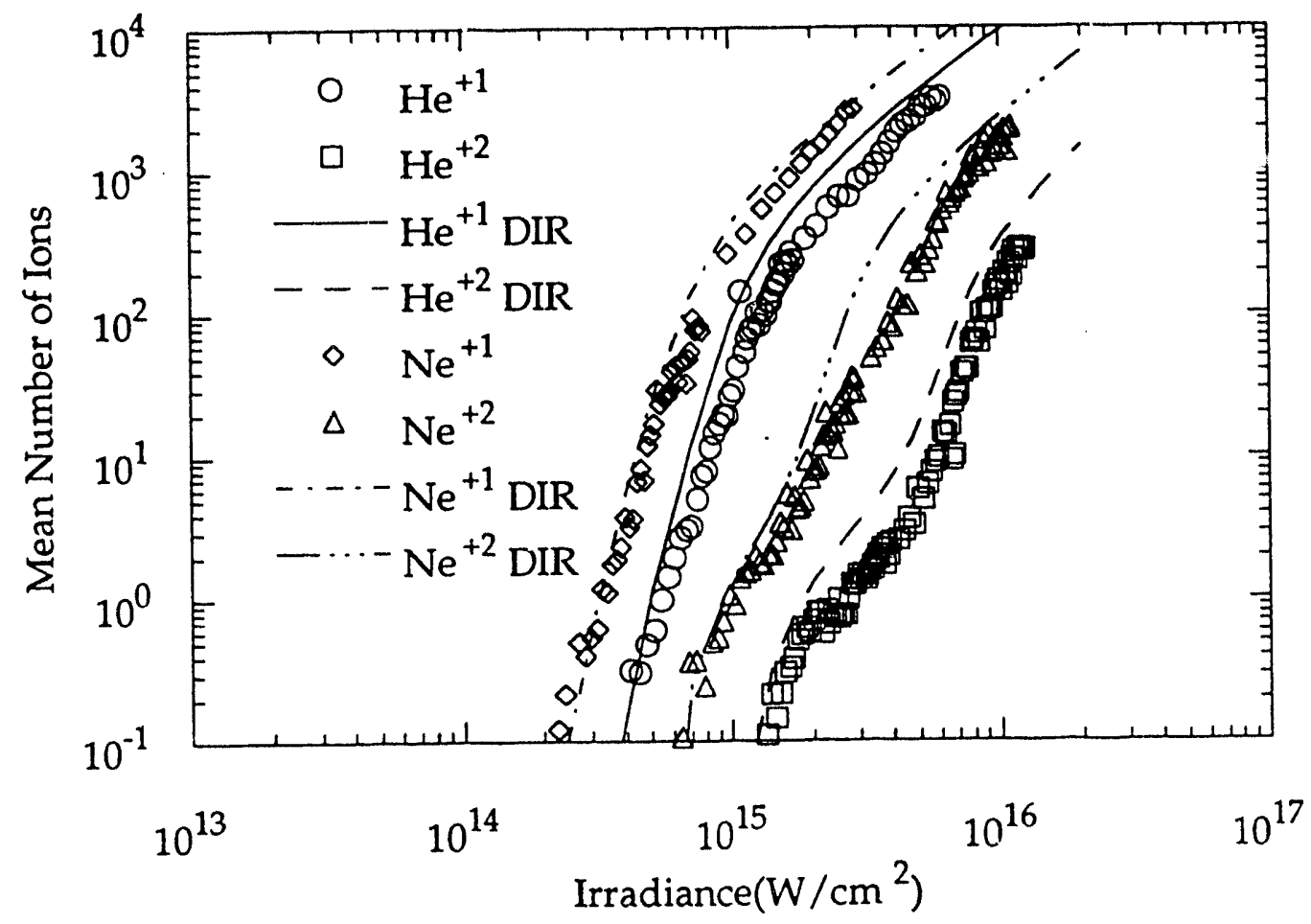

Figure 6.4.1 The data for linearly polarized $614-\mathrm{nm}$ pulses with a shake-off prediction(DIR) for helium and neon. The prediction uses the linear ADK rates and evaluates the direct component using $\frac{\Gamma_{0}^{\prime}}{\Gamma_{0}}=0.995$ when $\Gamma_{0} \geq R_{c}$ and one otherwise. For neon the critical instantaneous rate (irradiance) for the onset of a direct ionization component is $4 \times 10^{13} \mathrm{sec}^{-1}\left(5 \times 10^{14} \mathrm{~W} / \mathrm{cm}^{2}\right)$. The ADK saturation irradiances are listed in Table 3.7.6. 
determined by the energy dependent cross section. The maximum electron kinetic energy during the re-encounter with the singly charged ion is $3.17 U_{p}$ where $U_{p}$ is the laser pondermotive energy $\left(U_{p}=e^{2} E_{l}^{2} / 4 m \omega^{2}\right)$. This energy can exceed $100 \mathrm{eV}$ at $10^{15} \mathrm{~W} / \mathrm{cm}^{2}$ using $614 \mathrm{~nm}$ light. Figure 6.4 .2 shows the $614-\mathrm{nm}$ helium data for linear polarization plotted with the results of a rescattering calculation (based on the ADK ionization rates) for $0.6 \mu \mathrm{m}[65]$. The calculation used the published collision cross section of helium. The transverse spread of the

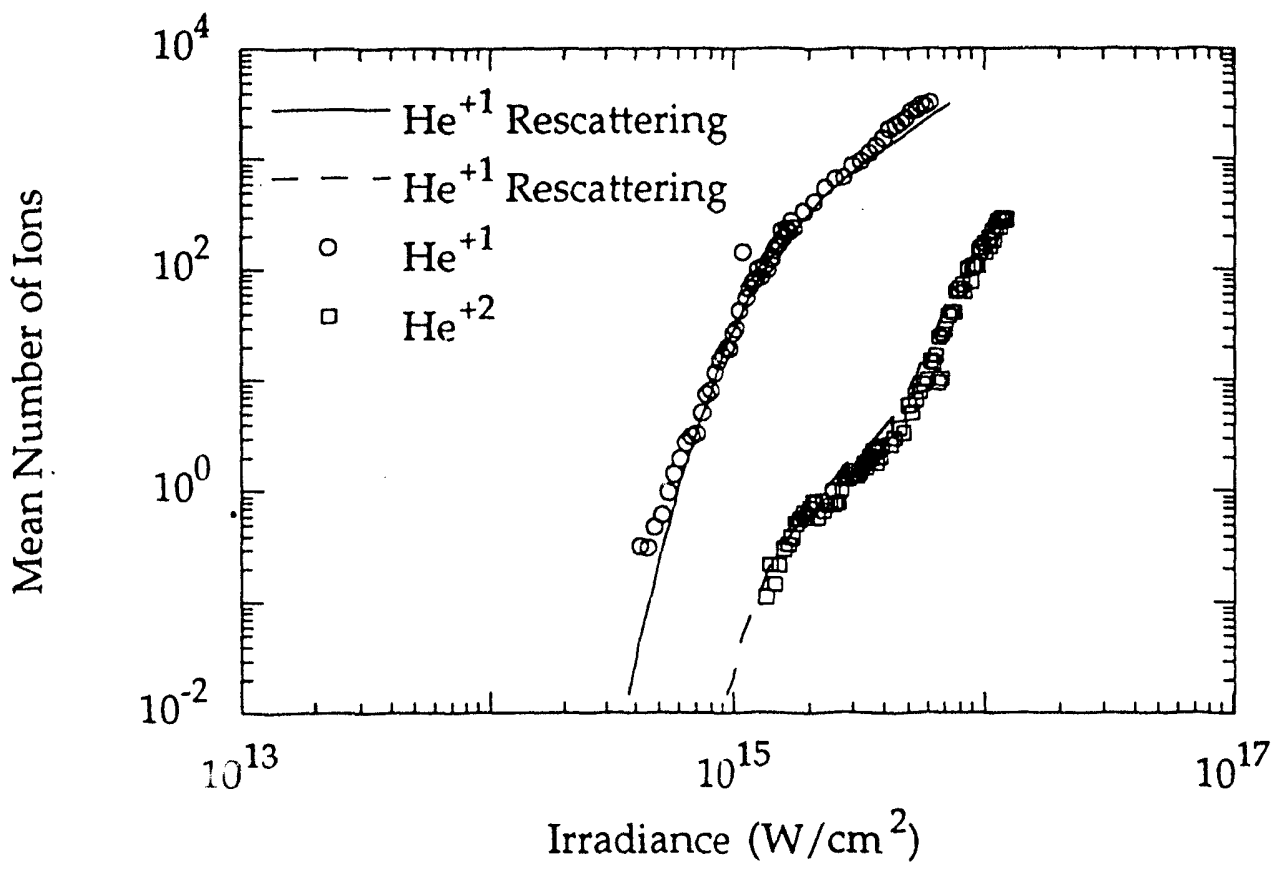

Figure 6.4.2 The 614-nm helium data for linear polarization plotted with the results of a rescattering calculation for $0.6 \mu \mathrm{m}$. Rescattering calculation courtesy of Paul Corkum.

returning electron's wave function was a free parameter. The agreement is excellent. Figure 6.4.3 shows the 614-nm neon data for linear polarization plotted with the results of a rescattering calculation for 625 -nm 100 -fs pulses. In this 


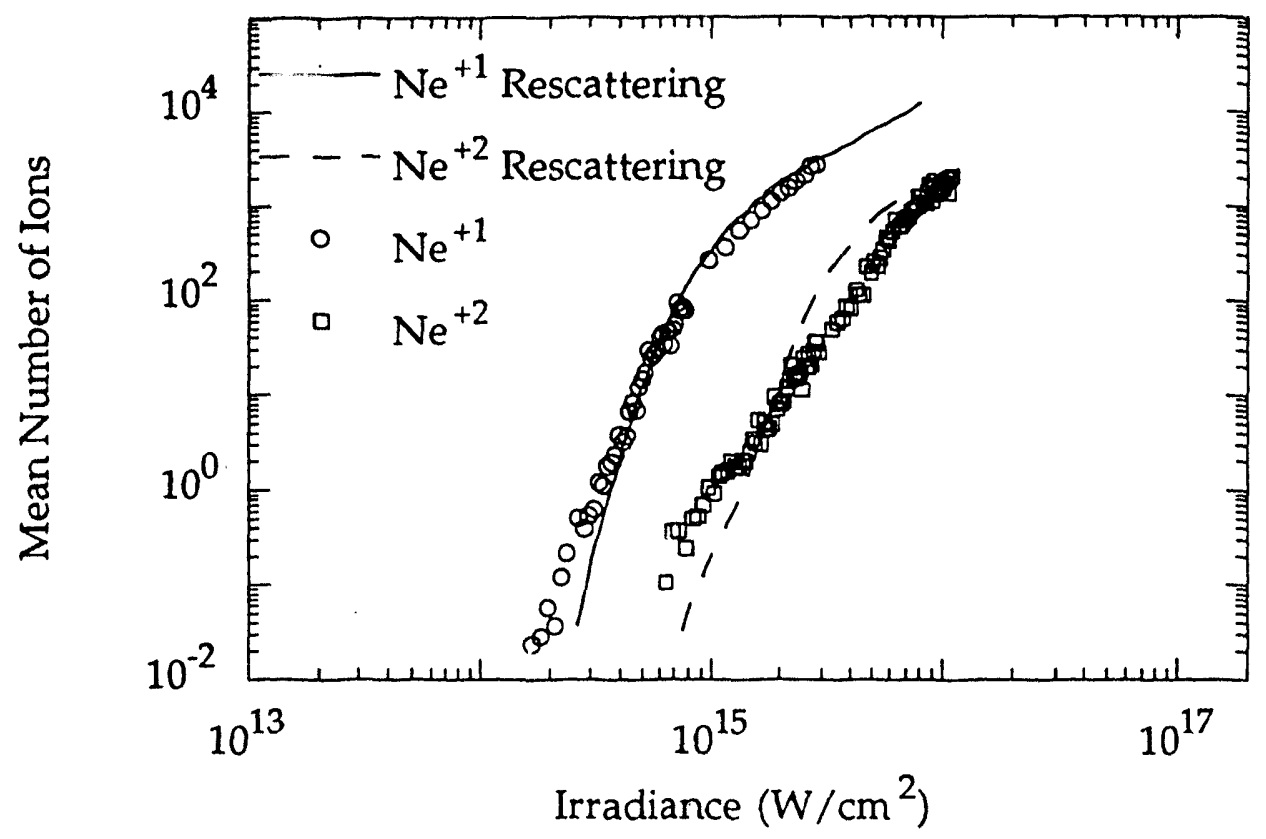

Figure 6.4.3 The 614-nm neon data for linear polarization plotted with the results of a rescattering calculation for $625-\mathrm{nm} 100-\mathrm{fs}$ pulses. (Rescattering calculation courtesy of Paul Corkum and Peter Dietrich.)

rescattering calculation[71], the transverse spread of the returning electron's wave function was fixed based on data from harmonic generation experiments. The calculation has been scaled to the $\mathrm{Ne}^{+1}$ yield. The calculation underestimates the magnitude of the enhancement by a factor of four. The under estimation is due to the use of field free collision cross sections and omission of inelastic excitations. The actual cross section in a strong field is expected to be higher due to the polarization of the atom along the field direction. The actual cross section would also need to include excitation cross sections to account for any excitation of the atom by the pulse before ionization. 
The wavelength dependence of this model is mainly due to the quadratic scaling of the pondermotive energy with $\lambda$. The polarization dependence stems from the possible transverse component of an electron trajectory induced by a perpendicular component of the laser field. In the circular case the amplitudes of the two perpendicular field orientations are equal, and the initially liberated electron can be steered away from the ion after tunneling. Quantum mechanically, the transverse spread of the electron wavepacket results in an effectively larger impact parameter. This reduces scattering and thus suppresses nonsequential ionization.

Figure 6.4.4 shows the $\mathrm{He}^{+2}$ and $\mathrm{Ne}^{+2}$ ion yields for linear polarization where the irradiances of the data have been scaled to align the sequential portion of the curves. The data are not aligned to the +1 charge states as was done for the comparison to theory because different species are compared. The nonsequential part of the $\mathrm{He}^{+2}$ yield rises at a lower irradiance relative to the sequential part than does the $\mathrm{Ne}^{+2}$ yield. The nonsequential parts of the curves saturate at approximately the same numbers of ions.

The species dependence of the rescattering model for fixed wavelength and pulse width is a function of the collisional ionization cross sections for species in strong fields and the return electron's energy distribution. Approximating the collision cross sections in strong field with the field free cross section, the measured electron collision ionization cross section at 1.1 times the ionization potential for the +1 charge state is $0.46 \times 10^{-18} \mathrm{~cm}^{2}$ for $\mathrm{He}^{+1}[72]$ and $2.1 \times 10^{-18} \mathrm{~cm}^{2}$ for $\mathrm{Ne}^{+1}[73]$. Note that classically the electron's return energy must be above the ionization potential for ionization to occur, so the values of the cross sections at 1.1 times the ionization potentials are given. 


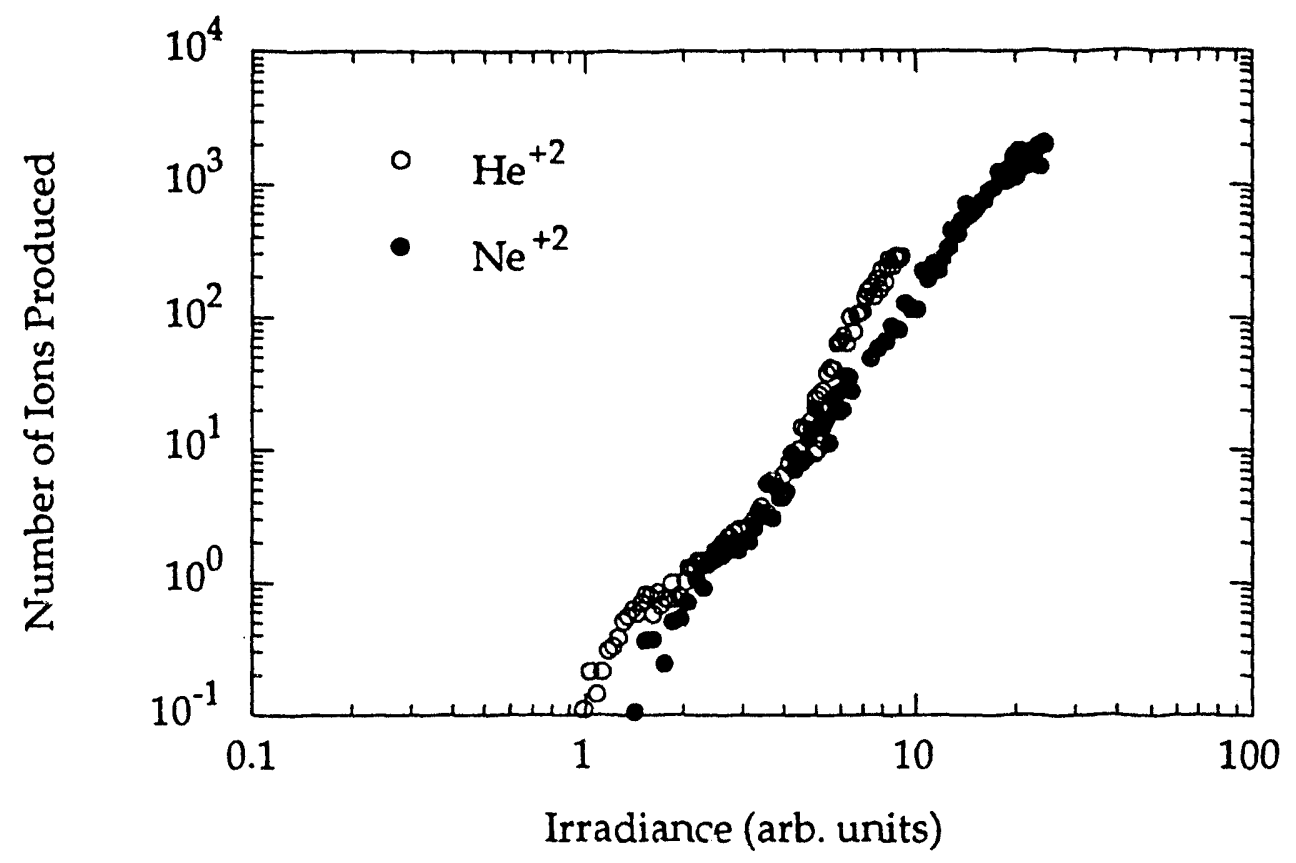

Figure 6.4.4 The $\mathrm{He}^{+2}$ and $\mathrm{Ne}^{+2}$ ion yields for linear polarization where the irradiances of the data have been scaled to align the sequential portion of the curves. 


\subsection{Circular Polarization Data at $614 \mathrm{~nm}$}

Since the two the direct models predict different dependences on polarization, I also measured the yields for circular polarization. Figure 6.5 .1 shows the helium and neon ion yields using circularly polarized pulses at $614-\mathrm{nm}$. Note that for

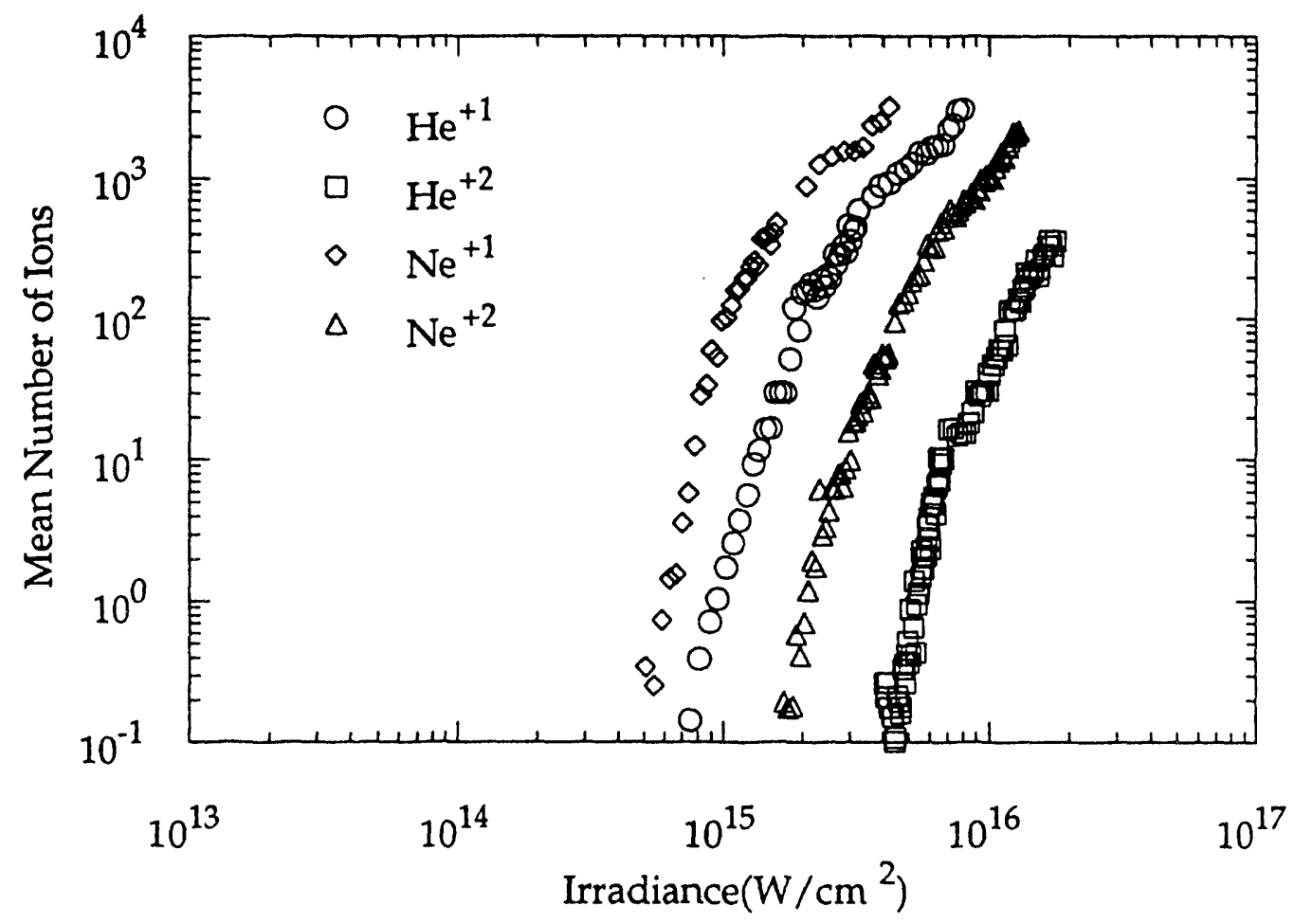

Figure 6.5.1 The helium and neon ion yields produced by circularly polarized 120-fs laser pulses at $614 \mathrm{~nm}$. The ADK threshold and saturation irradiances are listed in Table 3.7.6 for comparison.

$\mathrm{He}^{+1}$ and $\mathrm{Ne}^{+1}$ the appearance irradiances for circular are approximately twice the appearance irradiances for linear polarization. This confirms the tunneling picture for strong optical field ionization apart from any model. Tunneling with circular polarization differs from tunneling with linear polarization in two ways[27]. First, for a given laser irradiance, the peak electric field for circular polarization is smaller by the square root of two. This reduces the barrier 
suppression that allows the tunneling. Second, during the optical cycle the circular rate is constant because the field amplitude is constant (only its orientation changes in time). The linear rate, however, varies with the instantaneous field amplitude. For sequential ionization these differences result in a higher appearance irradiance for circular polarization. More importantly, the enhancements that were obvious for linear polarization data are absent in the circular polarization. In the multiphoton regime, suppression of ionization with circularly polarized light also occurs and has been attributed to emission of electrons in a single-step nonresonant coherent process rather than in a stepwise excitation[74].

Figure 6.5.2 compares the direct calculation with the 614-nm data for circular

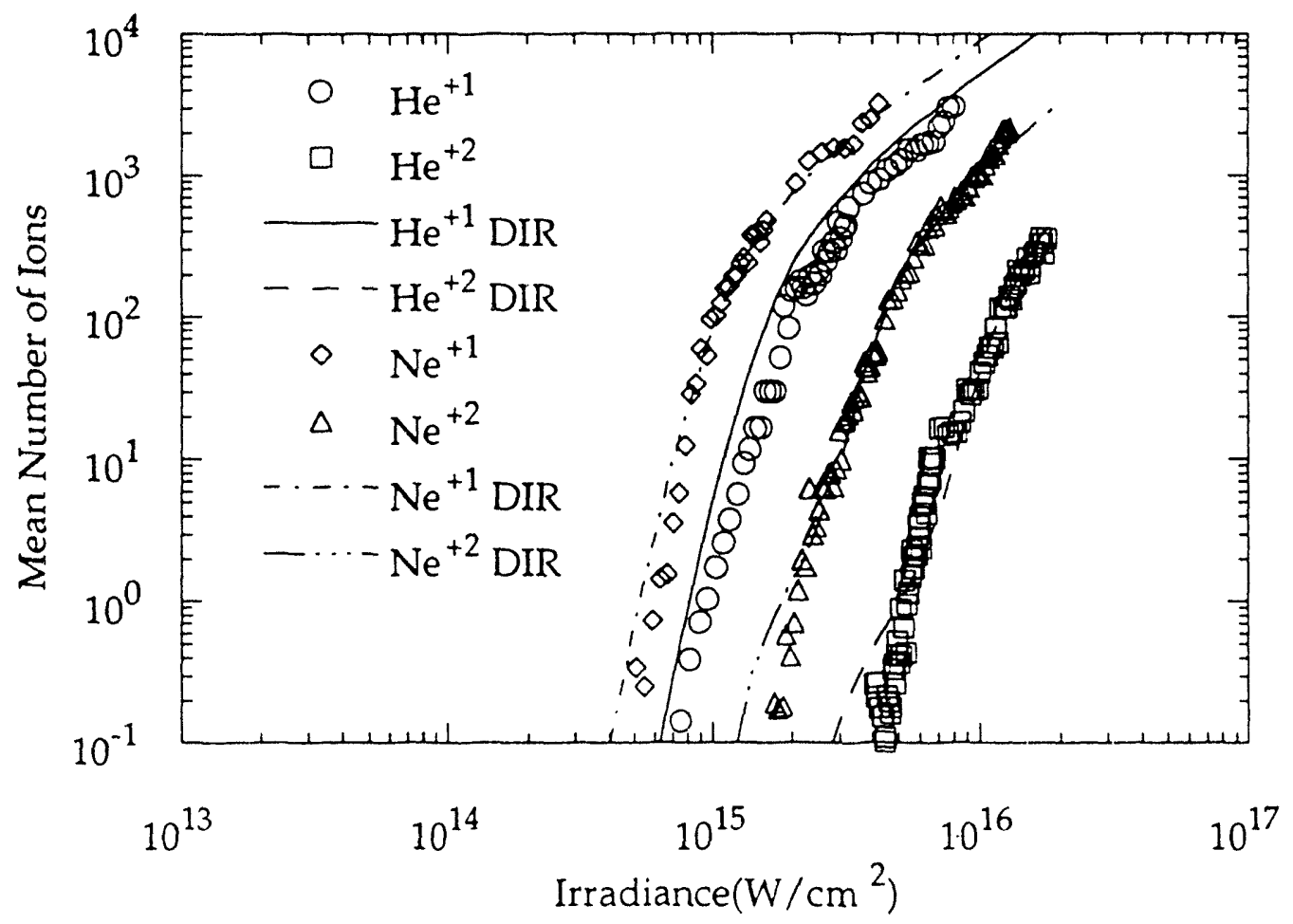

Figure 6.5.2 The direct calculation(DIR) of the ion yields with the 614-nm data for circular polarization. 
polarization. The rate scaling uses the circular ADK ionization rates and, for each gas, the same critical instantaneous rate, $R_{c}$, determined in the linear case. For circular polarization the corresponding critical irradiances for helium and neon are $2 \times 10^{15} \mathrm{~W} / \mathrm{cm}^{2}$ and $1 \times 10^{15} \mathrm{~W} / \mathrm{cm}^{2}$, respectively. The direct rate equations predict ionization enhancements that should be observable, but as noted above the yields exhibit no enhancements.

Figure 6.5.3 compares the sequential ADK prediction with the circular polarization data at $614-\mathrm{nm}$. The agreement is excellent, and sequential ionization completely describes the ionization for circularly polarized pulses. This is consistent with the rescattering mechanism for nonsequential ionization. Furthermore $E_{k}{ }^{3}$ is clear and is discussed later in Chapter 8.

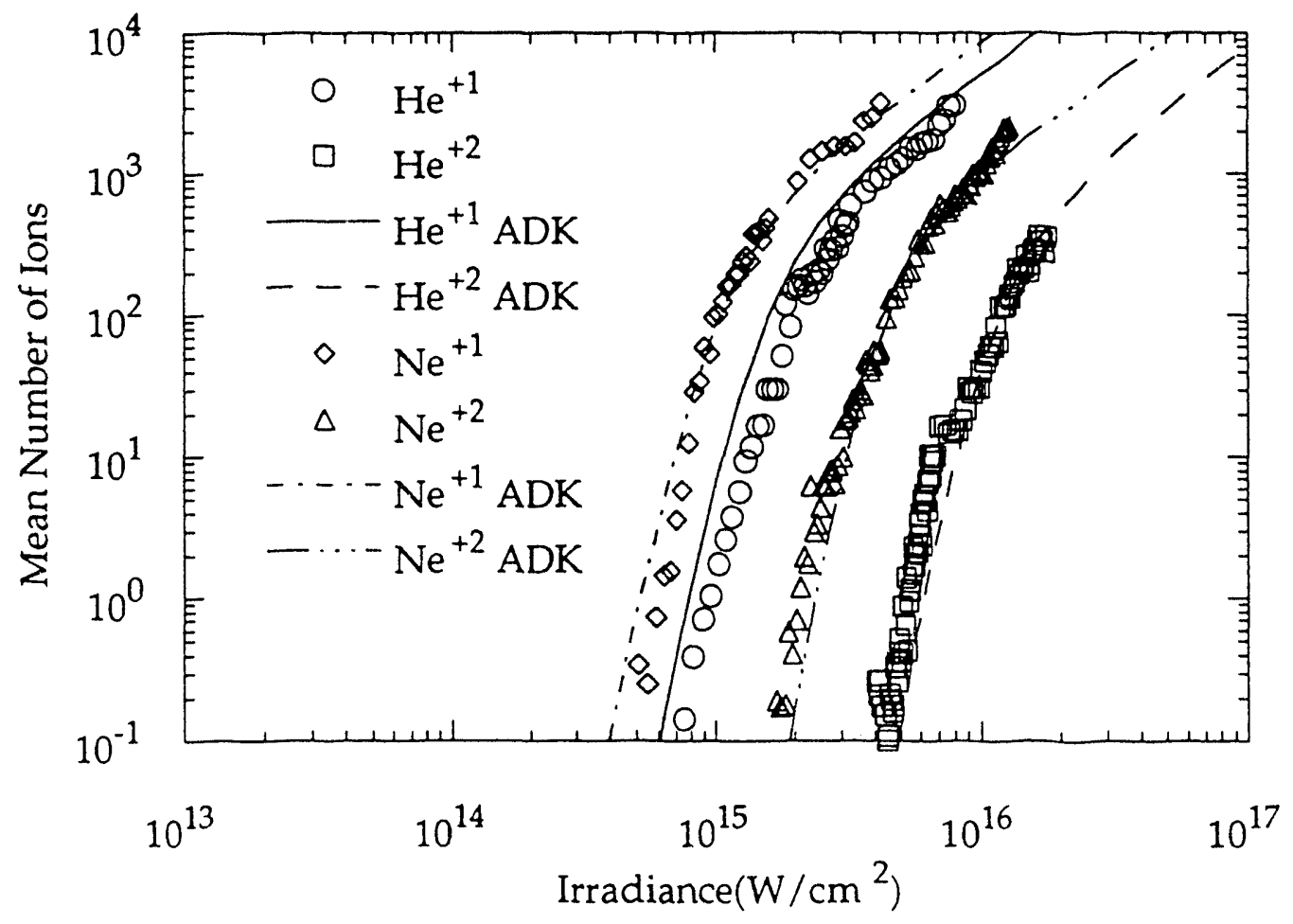

Figure 6.5.3 The 614-nm data for circular polarization and the corresponding ADK predictions. 


\subsection{Summary}

I measured the ion yields for helium and neon produced by 120 -fs pulses at $614 \mathrm{~nm}$. For linearly polarized pulses, the $\mathrm{He}^{+1}$ and $\mathrm{Ne}^{+1}$ the $\mathrm{ADK}$ sequential tunneling model predicts the yields well. For the singly ionized charge states, comparison of the appearance irradiances for linear and circular polarization supports the field scaling of the tunneling description. The $\mathrm{He}^{+2}$ and $\mathrm{Ne}^{+2}$ yields, however, ionize at lower irradiances than predicted by sequential tunneling, saturate at the same irradiances as their respective singly charged ions and then rise to join the ADK predictions. These features cannot be explained by sequential tunneling or resonances. Two nonperturbative direct nonsequential ionization processes have been considered that could have caused the linear polarization data: a shake-off mechanism and a rescattering mechanism. The data for circular polarization show a very strong polarization dependence-the enhancement disappears completely-that is consistent with the rescattering mechanism and inconsistent with the shake-off mechanism. The observed species scaling of the nonsequential part of the ion yields for linear polarization is also consistent with the scaling of the impact ionization cross sections that are used in the quasiclassical rescattering model. 


\section{CHAPTER 7-800NM IONIZATION DATA}

\subsection{Introduction}

The 614-nm results left some questions regarding the ellipticity, wavelength and species dependence of the enhanced production of the doubly ionized charge states. This chapter presents the measured ion yields for singly and doubly charged ions of $\mathrm{He}, \mathrm{Ne}$ and $\mathrm{Ar}$ created by linearly and circularly polarized laser pulses at $800 \mathrm{~nm}$ as was done for $\mathrm{He}$ and $\mathrm{Ne}$ at 614-nm in Chapter 6. The data show explicitly the ellipticity dependence of the ionization. A comparison is made between the enhancements observed at $614 \mathrm{~nm}$ and $800 \mathrm{~nm}$.

\subsection{Linear Polarization Data at $800 \mathrm{~nm}$}

Figure 7.2.1 shows the measured singly and doubly ionized helium, neon and argon ion yields versus peak irradiance for linearly polarized $800-\mathrm{nm}$ pulses. As noted previously the pulses have a Gaussian temporal profile with a FWHM of $130 \mathrm{fs}$. The focal spot has a FWHM of $9.6 \mu \mathrm{m}$. The data are scaled to a target pressure of $1 \times 10^{-3} \mathrm{~Pa}$. As the $800-\mathrm{nm}$ laser beam quality varies more than that of the 614-nm laser, the irradiance scale was set as follows. First, the appearance irradiances were measured for $\mathrm{He}^{+1}$ and $\mathrm{He}^{+2}$ produced by circularly polarized pulses. The irradiance for these measurements were obtained from the measured energy, focal profile and pulse width as was done for all the 614-nm data. During all subsequent measurements for other species or polarizations, the appearance energies for $\mathrm{He}^{+1}$ and $\mathrm{He}^{+2}$ were again measured for circular polarization. The relative appearance energies between the new species and the helium appearance energies then set the energy scale relative to the original measured irradiance scale for helium. The data for each charge state represents 800 to 1200 laser shots 


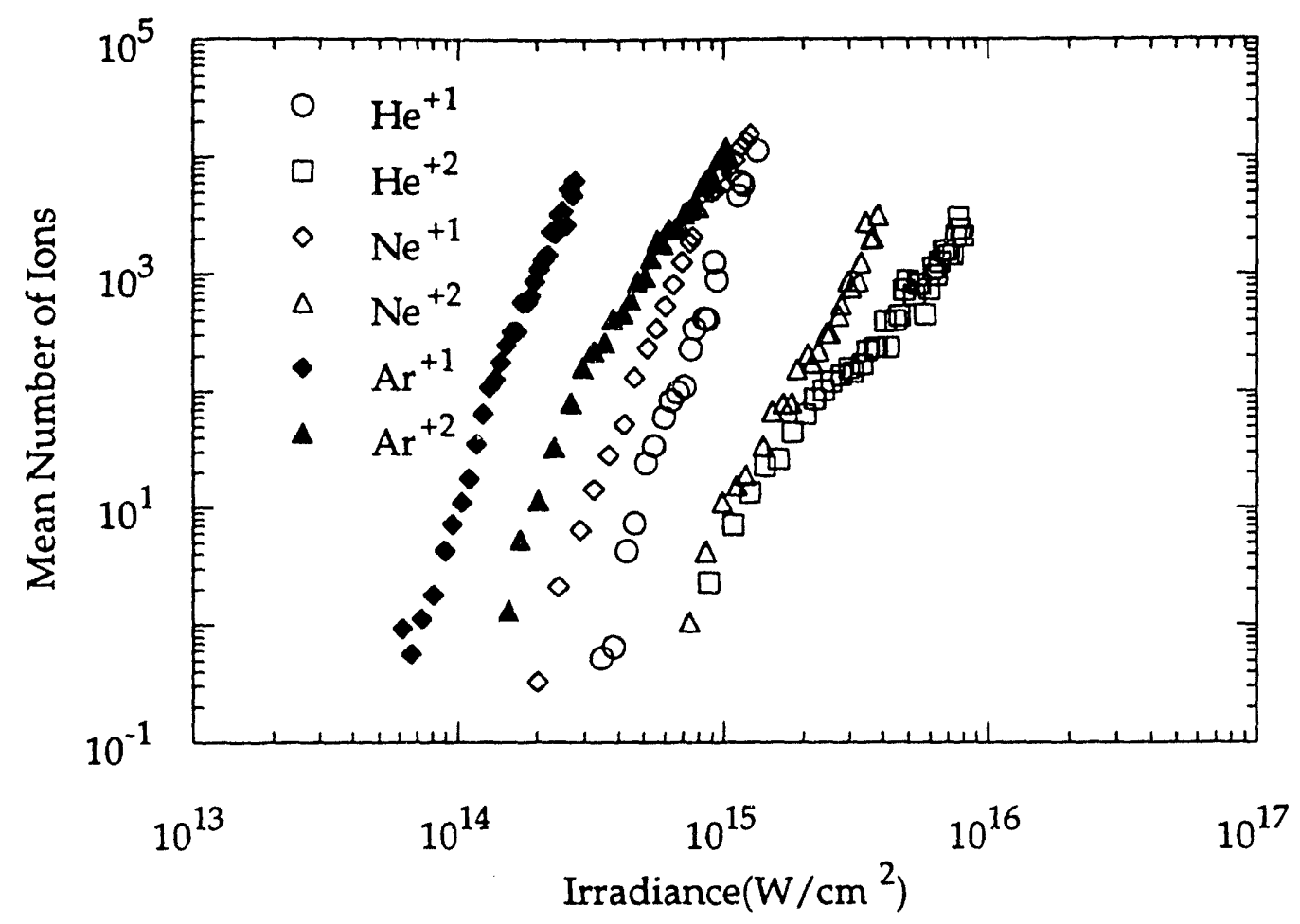

Figure 7.2.1 The helium, neon and argon ion yields produced by linearly polarized $130-\mathrm{fs}$ laser pulses at $800 \mathrm{~nm}$.

binned and averaged in approximately 40 evenly spaced bins. Fewer shots were used than for the 614-nm runs because the shot-to-shot energy fluctuations of the 800 -nm laser were generally only $10 \%$ as opposed to $100 \%$ for the $614-\mathrm{nm}$ laser. Measurements have been repeated several times and show no discernible shift in the relative positions.

As was true for the 614-nm data, the linear polarization data is obviously unusual even before comparing the data directly with a specific ionization model. Again the $\mathrm{He}^{+1}$ and $\mathrm{Ne}^{+1}$ the ion yields rise smoothly with irradiance. And again the $\mathrm{He}^{+2}$ and $\mathrm{Ne}^{+2}$ the ion yields rise, saturate and then rise again. In addition, the $\mathrm{Ar}^{+1}$ and $\mathrm{Ar}^{+2}$ ion yields rise smoothly with irradiance and then 
begin to saturate. The $\mathrm{Ar}^{+2}$ ion yield, however, rises before the $\mathrm{Ne}^{+1}$ ion yield. This is obviously unusual because $\mathrm{Ar}^{+1}$ has a higher ionization potential at 27.63 $\mathrm{eV}$ than the $21.56 \mathrm{eV}$ ionization potential of neutral neon. Thus the sequential prediction would be for $\mathrm{Ar}^{+2}$ to appear at a higher irradiance than

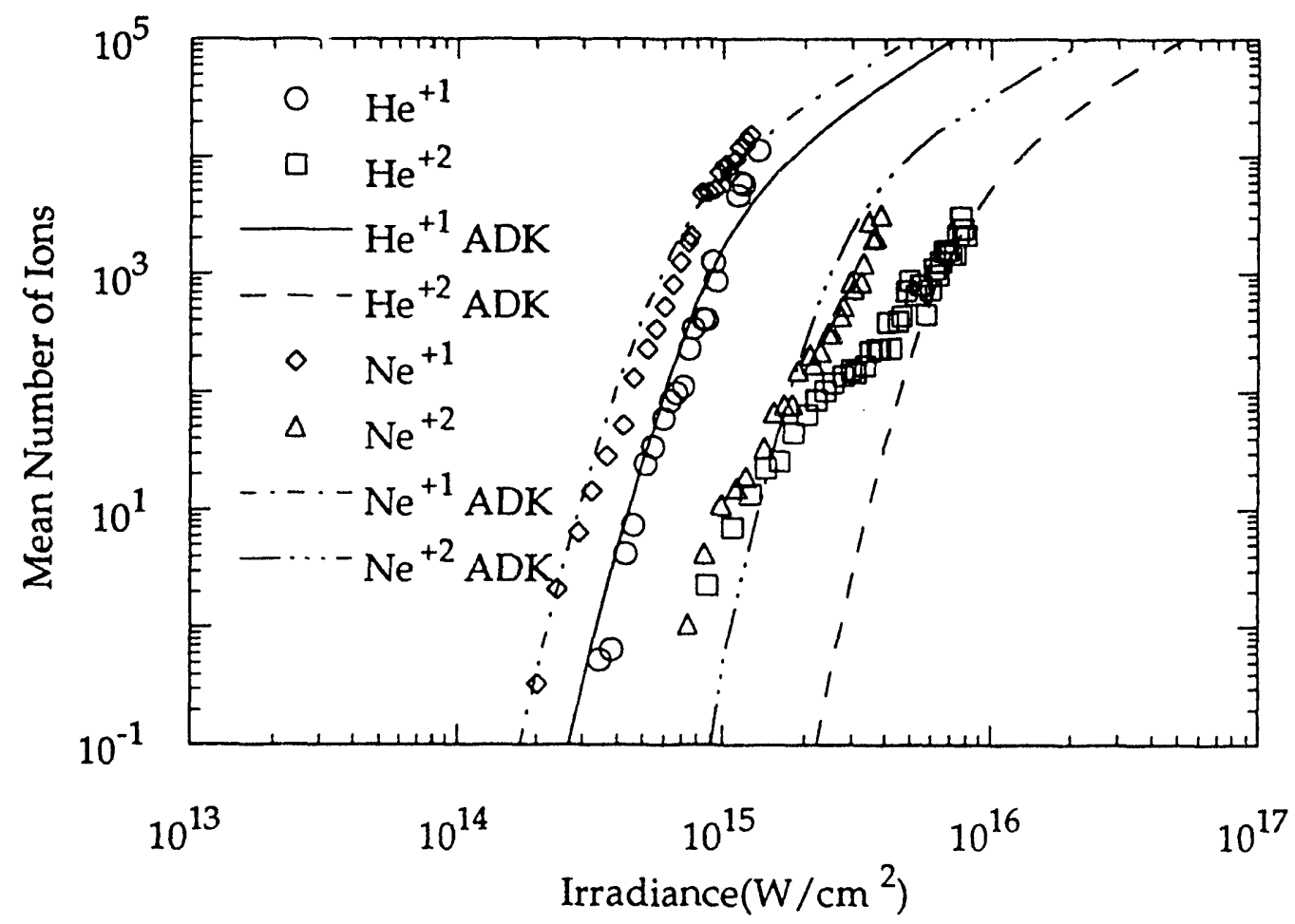

Figure 7.2.2 The helium and neon ion yields produced by linearly polarized 130-fs laser pulses at $800 \mathrm{~nm}$ superimposed on the sequential ADK predictions. The ADK threshold and saturation irradiances are listed in Table 3.7.6 for comparison.

$\mathrm{Ne}^{+1}$. As at $614 \mathrm{~nm}$, the discrepancies between the linear polarization data and the sequential predictions for the doubly ionized charge states become more apparent when the data is compared to the ADK predictions. Figures 7.2.2 and 7.2.3 show the linear polarization data superimposed on the predictions of the sequential ADK theory. The data for argon has been plotted separately since the 
$\mathrm{Ar}^{+2}$ calculation and $\mathrm{Ne}^{+2}$ yields are very close together and would obscure each other if plotted together. Again as at 614-nm, the figure shows excellent agreement for the singly ionized charge states to within the uncertainty in the irradiance. The doubly ionized yields, however, appear at irradiances significantly lower than the ADK appearance irradiance. The yields then rise and

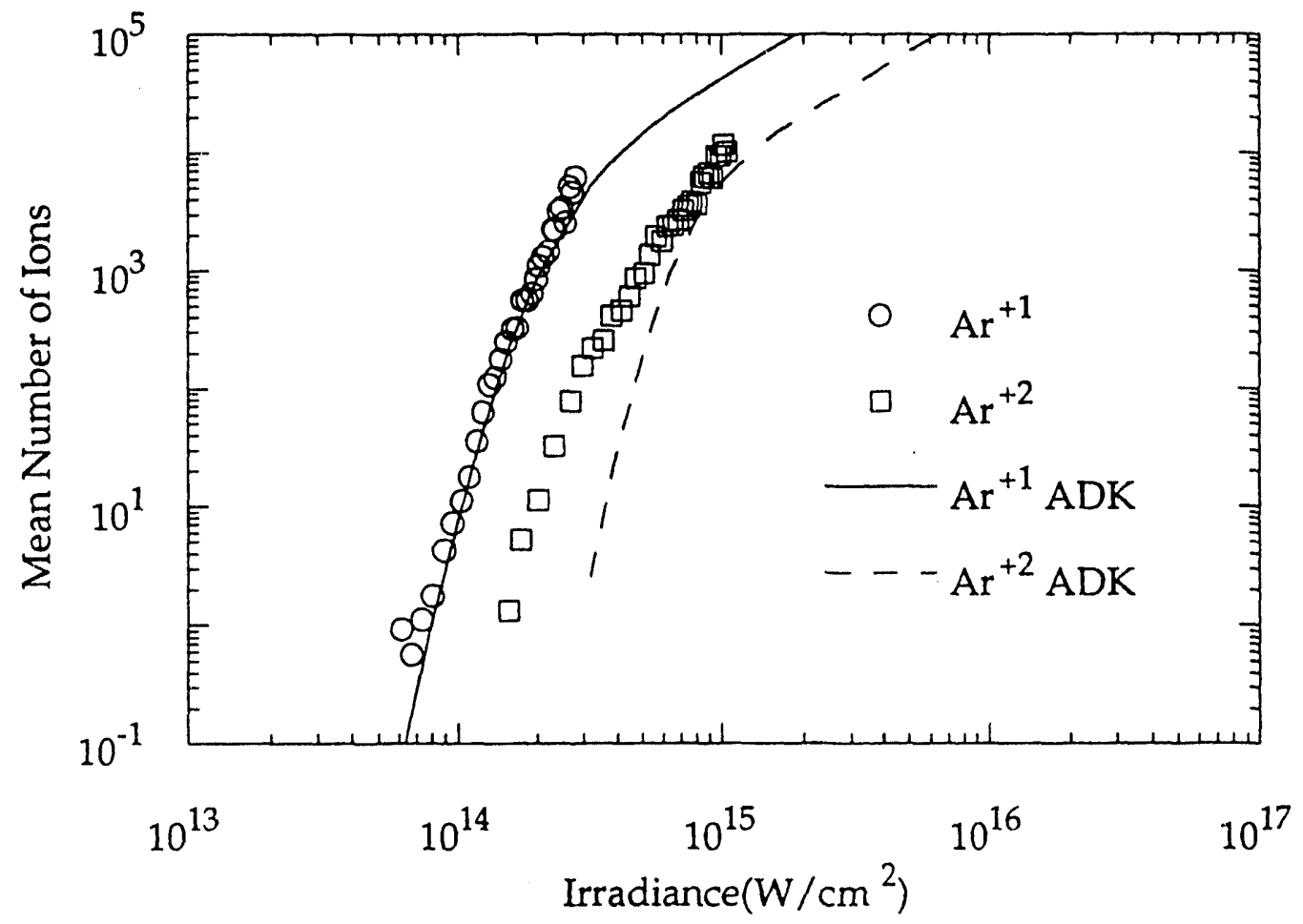

Figure 7.2.3 The argon ion yields produced by linearly polarized 130 -fs laser pulses at $800 \mathrm{~nm}$ superimposed on the sequential ADK predictions.

saturate at the same irradiances as predicted for the singly charged states. Eventually the yield rejoins the sequential ADK predictions for the doubly ionized ion yields. 
Figure 7.2.4 compares the neon ion yields to the prediction of the ADK model with rescattering of the first electron removed as proposed by Corkum[75]. The rescattering calculations have been scaled in number and irradiance to the $\mathrm{Ne}^{+1}$ data. The enhanced portion of the ion yield is higher in the data than in the theory by a factor on the order of 2 as was observed at for the 614-nm data. This underestimation of the actual enhancement was also observed in neon at 625 rum[71]. Again this difference is expected since the e-2e collision cross section used in the calculation is for collisions in the absence of applied electric fields or

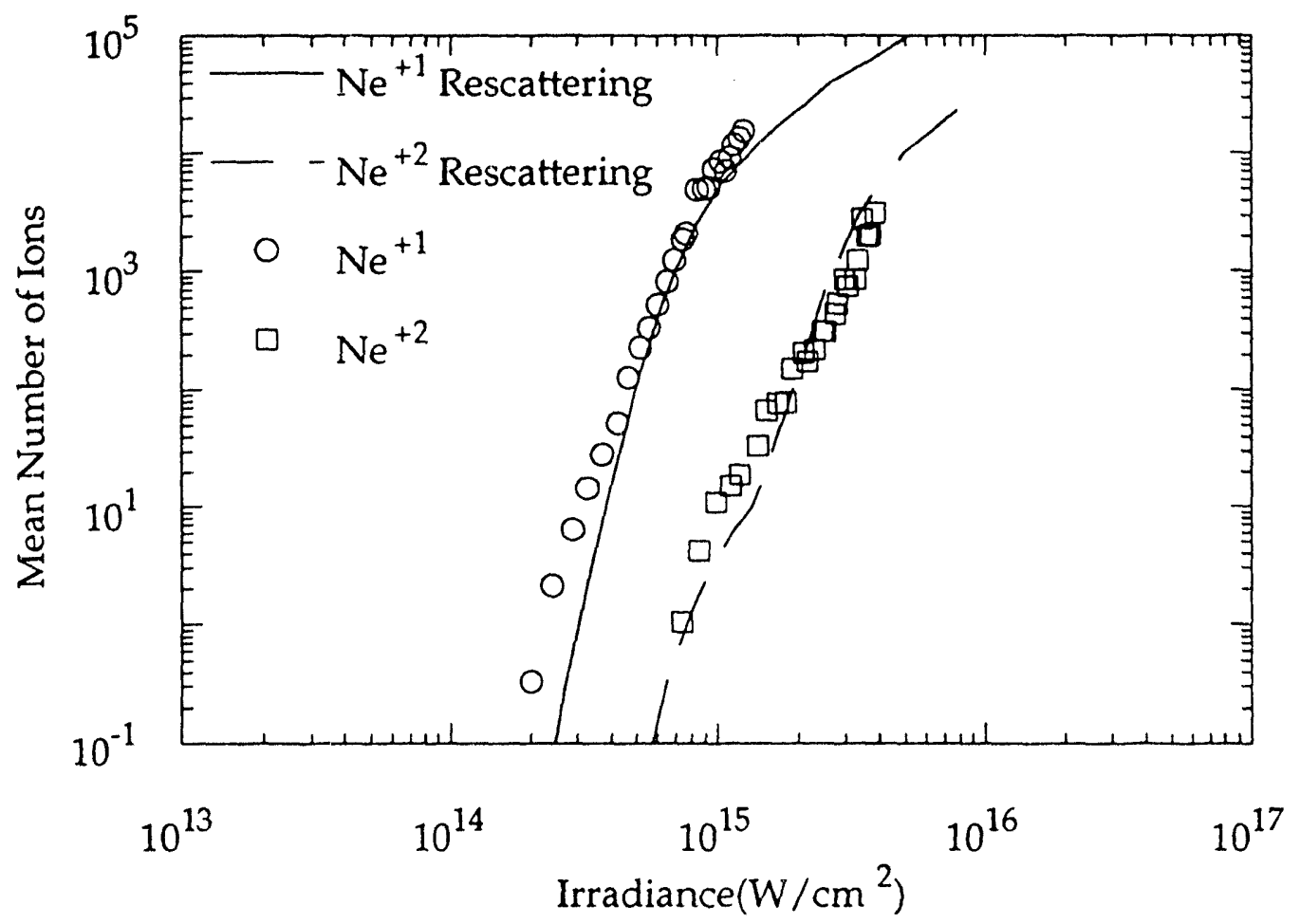

Figure 7.2.4 The neon ion yields produced by linearly polarized 130 -fs laser pulses at $800 \mathrm{~nm}$ superimposed on the predictions of the ADK model including rescattering of the first electron removed[75]. 
excitations. Qualitatively, however, the agreement is good and establishes the rescattering model as a likely explanation of the enhancements. The species dependence of the nonsequential part of the $\mathrm{He}^{+2}$ and $\mathrm{Ne}^{+2}$ ion yields for linear polarization is shown in Figure 7.2.5. The irradiances of the yield data have been scaled to align the sequential part of the curves. As for $614 \mathrm{~nm}$, the $\mathrm{He}^{+2}$ rises at a lower irradiance relative to the sequential part of the yield than does $\mathrm{Ne}^{+2}$. At $800 \mathrm{~nm}$ the $\mathrm{He}^{+2}$ yield remains higher than the $\mathrm{Ne}^{+2}$ yield until the sequential rise of the yield occurs.

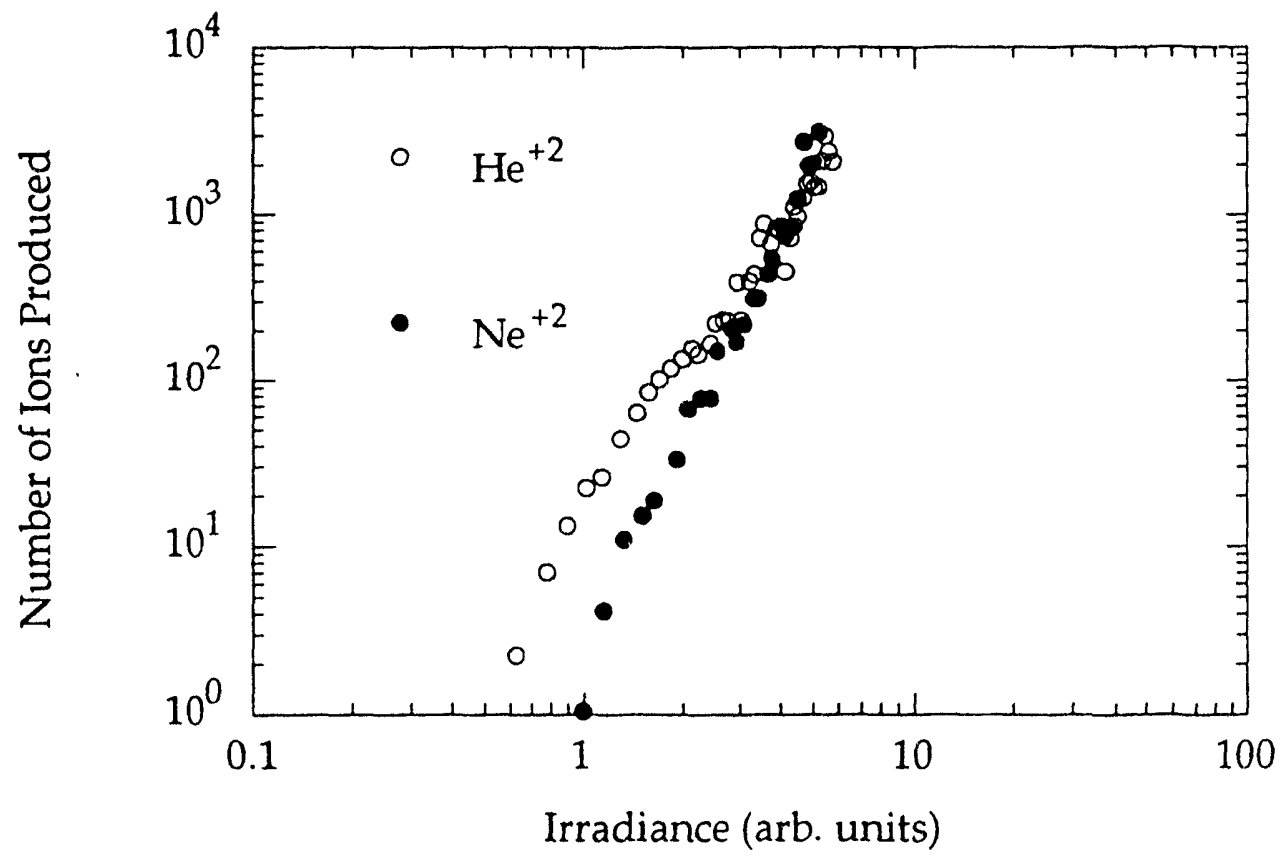

Figure 7.2.5 The irradiance normalized ion yields for $\mathrm{He}^{+2}$ and $\mathrm{Ne}^{+2}$ for linear polarization at $800 \mathrm{~nm}$. The irradiance is normalized to align the sequential part of the yield curves. 


\subsection{Circular Polarization Data at $800 \mathrm{~nm}$}

Figure 7.3.1 shows the measured ion yields for circular polarization. For circular polarization, all the charge states rise smoothly and then volume saturate (not enough data was taken to see saturation in $\mathrm{Ar}^{+1}$ ). In addition, the $\mathrm{Ar}^{+2}$ yield now appears at a higher irradiance than the $\mathrm{Ne}^{+1}$ yield as predicted by the ionization potentials. The same effects seen for $614 \mathrm{~nm}$ in helium and neon are observed for helium neon and argon at $800 \mathrm{~nm}$. Large enhancements to the doubly charged ion yields that were apparent for linear polarization disappear for circular polarization.

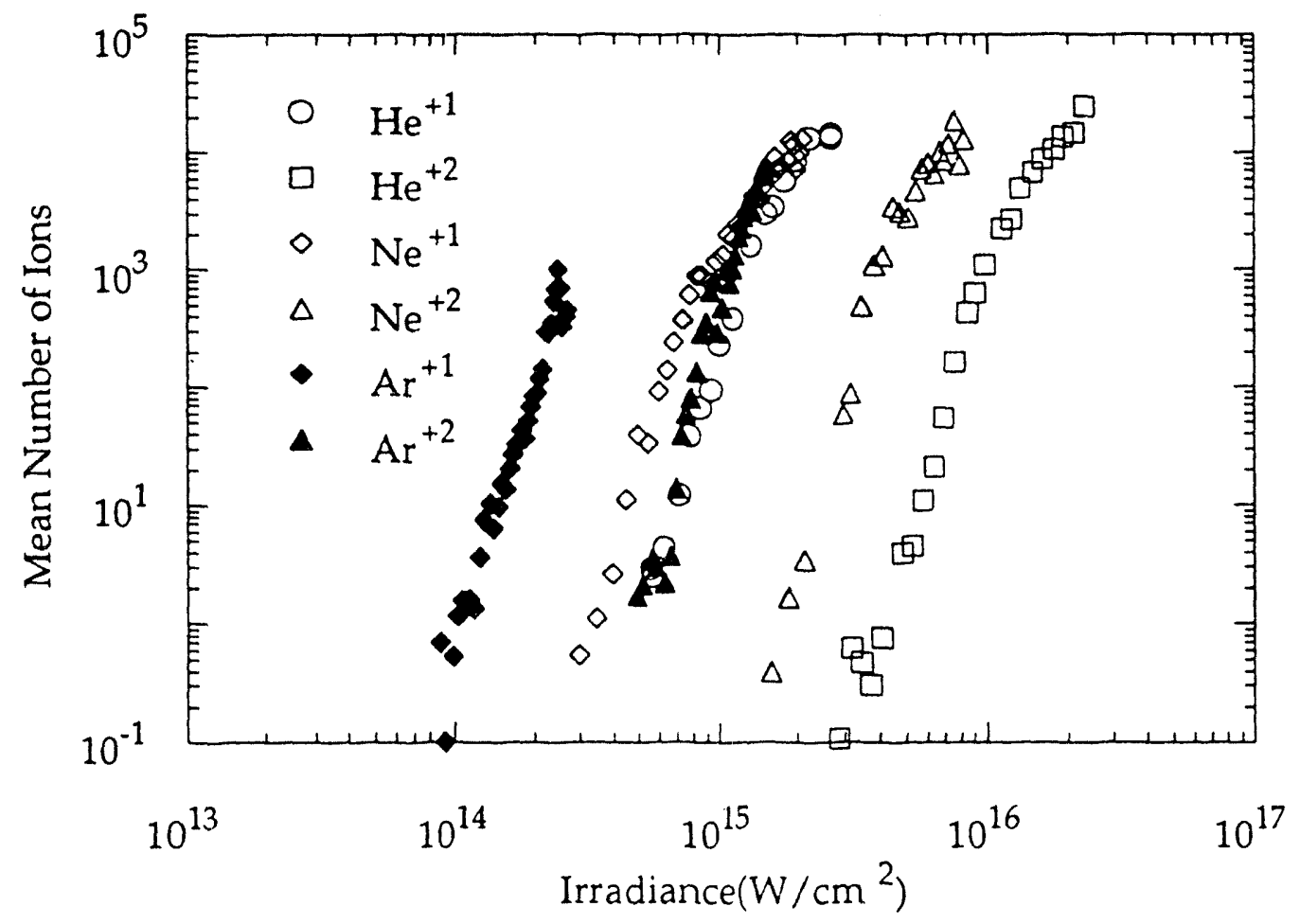

Figure 7.3.1 The helium, neon and argon ion yields produced by circularly polarized $130-$ fs laser pulses at $800 \mathrm{~nm}$. 


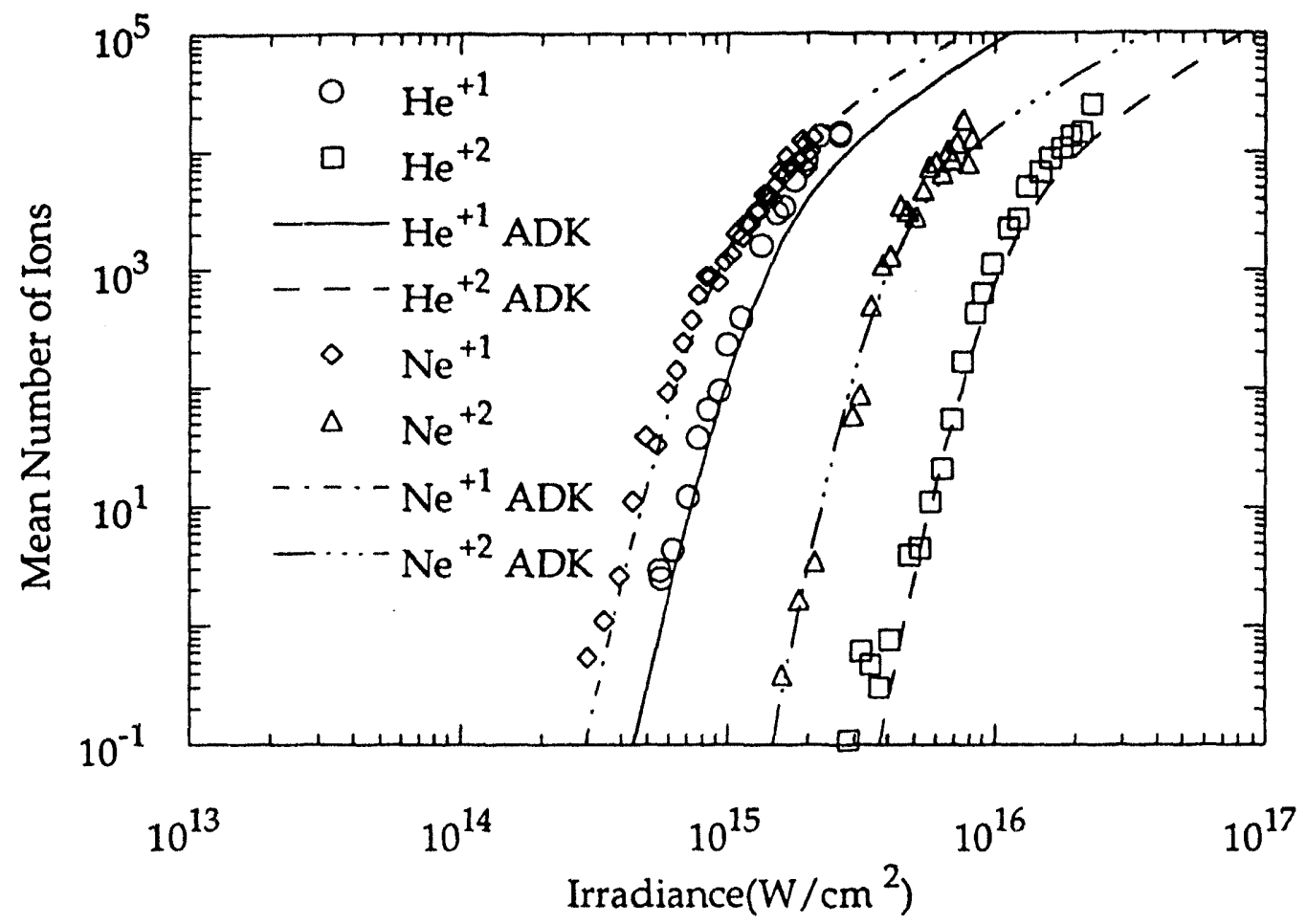

Figure 7.3.2 The helium and neon ion yields produced by circularly polarized 130-fs laser pulses at $800 \mathrm{~nm}$ superimposed on the sequential ADK predictions.

Figures 7.3.2 and 7.3.3 confirm that for circular polarization the ionization follows sequential tunneling theory. Figure 7.3.2 shows the helium and neon ion yields for circular polarization and the ADK predictions. Figure 7.3.3 shows the argon ion yields for circular polarization and the ADK predictions. Again the argon data has been separated from the helium and neon data for clarity of presentation. For helium and neon the agreement is excellent. For argon the agreement is good although insufficient data was taken (due to experimenter error) to see if the yields saturate as predicted. 


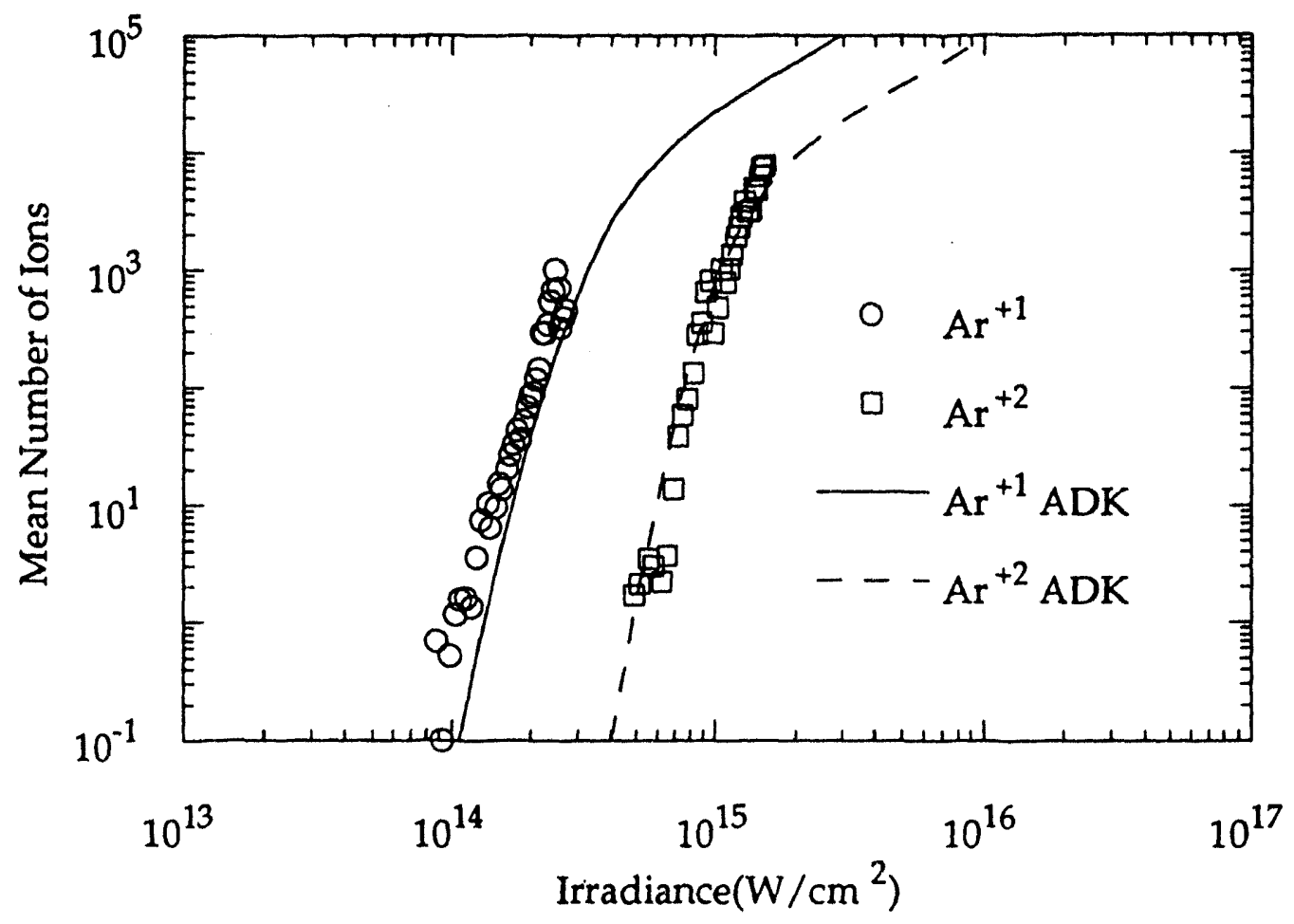

Figure 7.3.3 The argon ion yields produced by circularly polarized 130 -fs laser pulses at $800 \mathrm{~nm}$ superimposed on the sequential ADK predictions.

\subsection{Data for Other Ellipticities at $800 \mathrm{~nm}$}

While the circular polarization data confirms that the enhancements are strongly polarization dependent a more detailed look at the polarization dependence produced more quantitative results for the model comparison. Figure 7.4.1 shows the $\mathrm{He}^{+2}$ yields at ellipticities of 0.0 (linear polarization) , $0.2,0.4$ and 1.0 (circular polarization). The number of ions attributable to nonsequential ionization is nearly an order of magnitude smaller for an ellipticity of 0.2 than for linear polarization, and the enhancement completely vanishes for an ellipticity of 0.4 . 


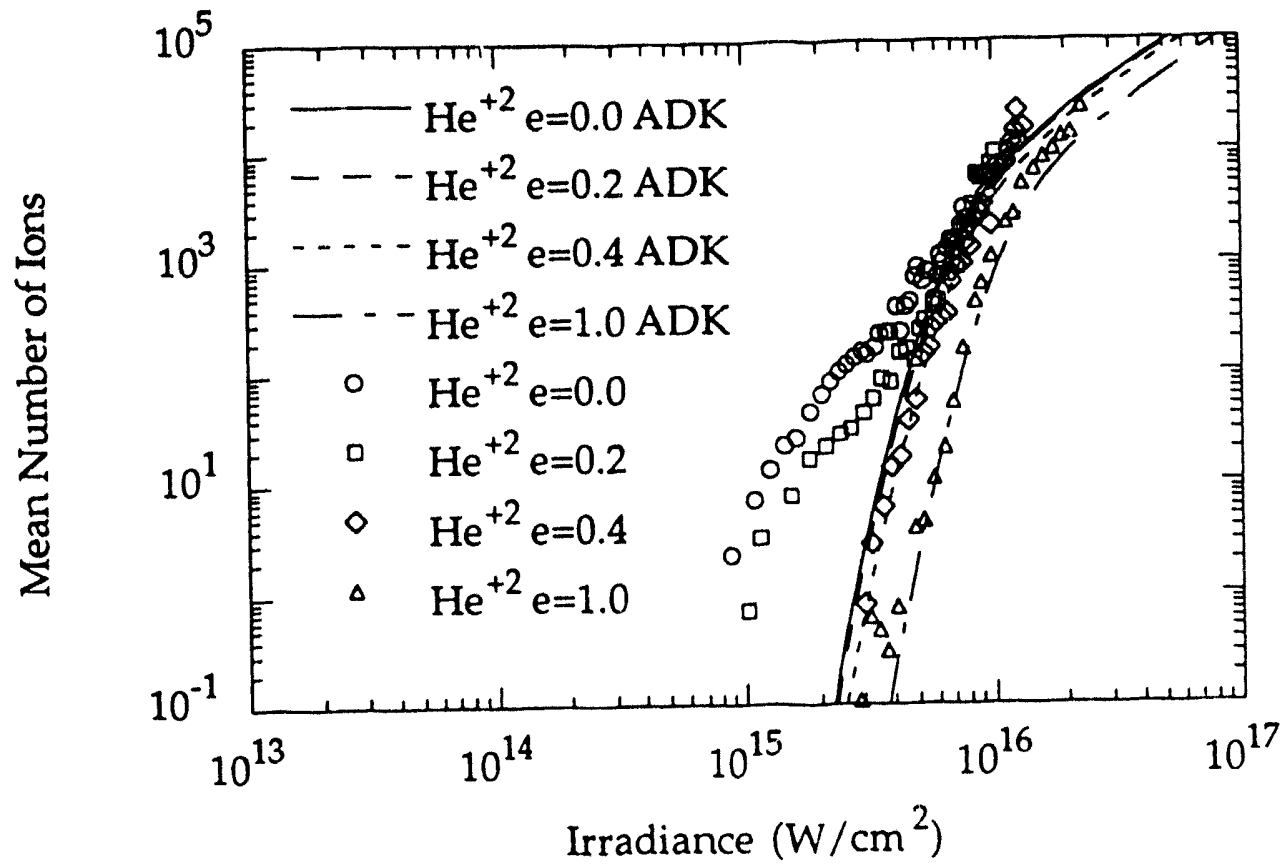

Figure 7.4.1 The doubly ionized helium yield produced by $130 \mathrm{fs}$ pulses at $800 \mathrm{~nm}$ with ellipticities of $e=0.0,0.2,0.4$, and 1.0

The decrease is rapid, but it is not as rapid as some initial estimates for the rescattering theory[65]. The decrease is consistent with other recent measurements of ionization and harmonic generation in neon at $625 \mathrm{~nm}[71]$.

Figure 7.4.2 shows another way of looking at the ellipticity dependence-that is to plot the number of $\mathrm{He}^{+2}$ ions versus ellipticity at a fixed irradiance. The two irradiances used are $1.2 \times 10^{16} \mathrm{~W} / \mathrm{cm}^{2}$ and $2.0 \times 10^{15} \mathrm{~W} / \mathrm{cm}^{2}$. A look at Figure 7.4.1 shows that $1.2 \times 10^{16} \mathrm{~W} / \mathrm{cm}^{2}$ is in the volume saturation regime and that $2.0 \times 10^{15}$ $\mathrm{W} / \mathrm{cm}^{2}$ is in the regime attributed to nonsequential ionization. While the number of ions agrees with the ADK theory for $1.2 \times 10^{16} \mathrm{~W} / \mathrm{cm}^{2}$, the measured number of ions is much higher than the ADK prediction for $2.0 \times 10^{15} \mathrm{~W} / \mathrm{cm}^{2}$. For linear 
polarization (ellipticity of 0.0 ), the ADK prediction underestimates the number of ions by three orders of magnitude. The measured yield also falls off much more

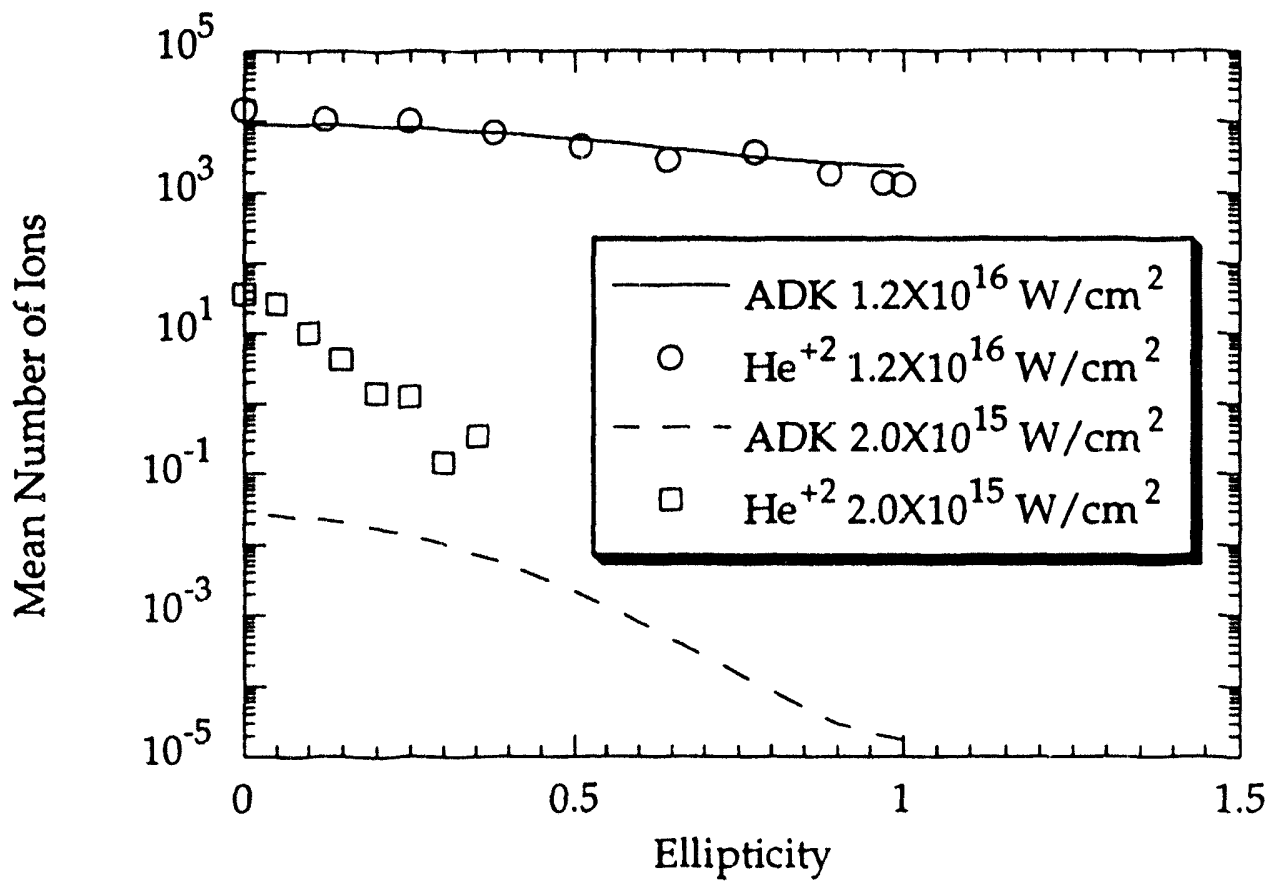

Figure 7.4.2 The doubly ionized helium yield as a function of ellipticity superimposed on the ADK predictions. Two peak irradiances are shown. $1.2 \times 10^{16} \mathrm{~W} / \mathrm{cm}^{2}$ and $2.0 \times 10^{15} \mathrm{~W} / \mathrm{cm}^{2}$.

quickly with increasing ellipticity than the ADK prediction. By an ellipticity of 0.3 the measured yield has decreased by two orders of magnitude. The decrease according to the ADK prediction should be only a factor of 3 . Thus the ionization is again seen to be incompatible with sequential ionization. Also the portion of the yield attributed to nonsequential ionization is a strong function of polarization. This suggests strongly that the physical process responsible for the nonsequential enhancement is different from the basic turneling process considered in the sequential part of the yield. As a comparison with the 
polarization dependence of the rescattering model, Figure 7.4.3 shows the $\mathrm{He}^{+2}$ yield data versus ellipticity-for $2.0 \times 10^{15} \mathrm{~W} / \mathrm{cm}^{2}$ and linear polarization at 800 $\mathrm{nm}$-that was shown in Figure 7.4.2 plotted with a rescattering prediction for $\mathrm{Ne}^{+2}$ at $625 \mathrm{~nm}$. The rescattering calculation is for $100 \mathrm{fs}$ pulses at irradiances of $9 \times 10^{14} \mathrm{~W} / \mathrm{cm}^{2}$. The yields are normalized at an ellipticity of 0 . The comparison while crude does show that the model and data show similar decreases in the number of ions produced of over an order of magnitude as the ellipticity increases from zero to 0.2 . As noted above these are much more rapid decreases than predicted by the sequential ADK model. A more accurate comparison of the

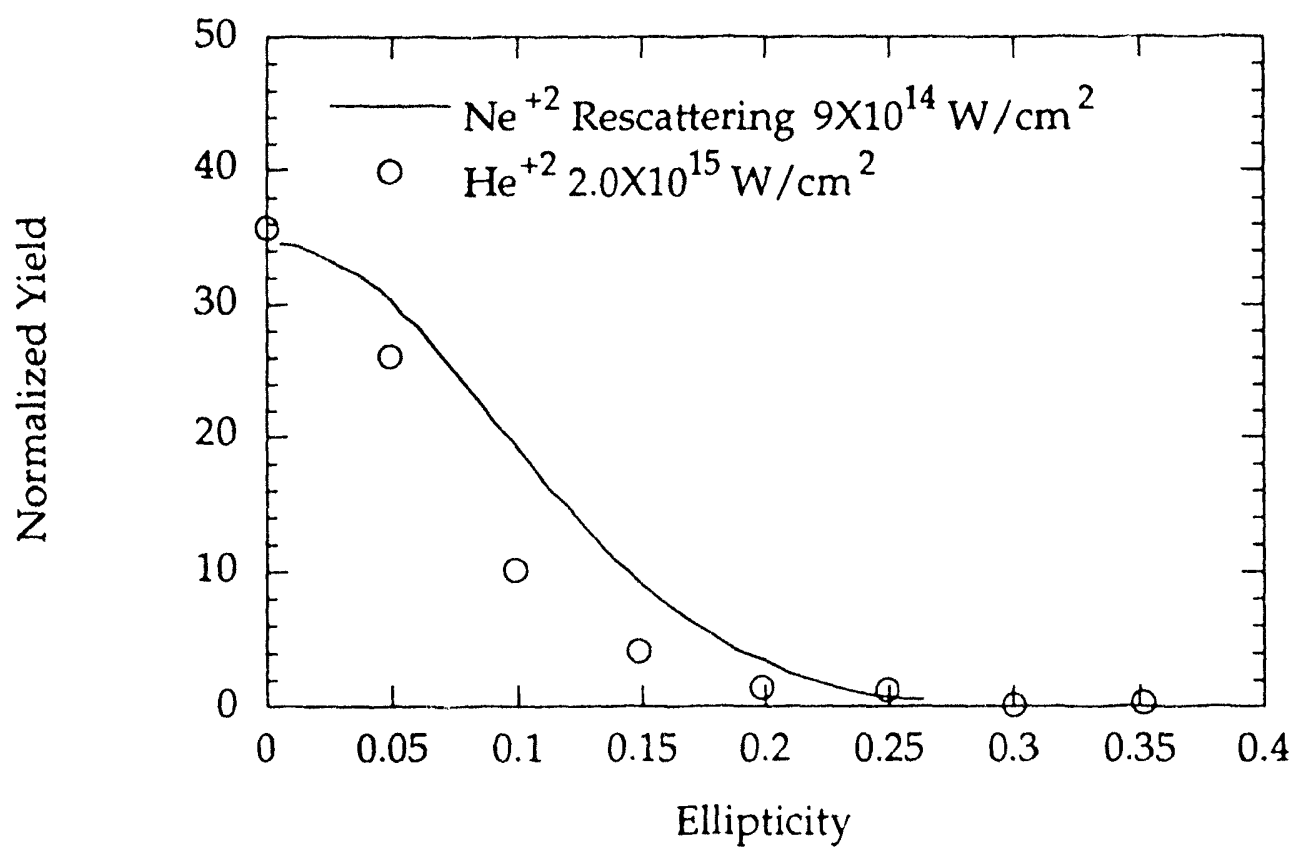

Figure 7.4.3 The $\mathrm{He}^{+2}$ yield data at $800 \mathrm{~nm}$ versus ellipticity plotted with a rescattering prediction for $\mathrm{Ne}^{+2}$ at $625 \mathrm{~nm}$. The data is for $2.0 \times 10^{15} \mathrm{~W} / \mathrm{cm}^{2}$ and linear polarization. The rescattering calculation is for $100 \mathrm{fs}$ pulses at irradiances of $9 \times 10^{14} \mathrm{~W} / \mathrm{cm}^{2}$. 
ellipticity dependence of the rescattering model with data is made in reference [71] that shows good agreement.

As an experimental note, this strong polarization dependence makes careful characterization of optics important. Some windows made of crystalline materials such as magnesium fluoride can alter the polarization enough to destroy any nonsequential effects if the optic axis of the window is not exactly parallel to the incident wave. The windows used in this experiment were fused quartz, but measurements using a single crystal magnesium fluoride window showed that alignment deviations as small as $1.5^{\circ}$ could eliminate any contribution due to nonsequential ionization.

In addition, the polarization dependence of the enhancements to the ion yields provides an important tool in monitoring the irradiance of a pulse using tunneling ion yields. The appearance irradiances for linear polarization are not easily calculable due to the enhanced ionization. Using circular polarization eliminates the enhancements and allows the appearance irradiances to be calculated simply from the ADK model thereby providing a simple monitor of the peak irradiance of the laser pulse. Applications of this technique for linear polarization are doomed although such applications are made frequently.

\subsection{Wavelength Comparison}

One difference between the rescattering model and the shake-off model is in their predictions for the wavelength dependence. The shake-off model is expected to be largely wavelength independent because it is a function of the rate of energy deposited in the atom. The rescattering model, however, relies on the dynamics of the laser cycle and should depend on the wavelength. The maximum energy of the rescattering electron is estimated to be $3.17 U_{i},[71]$ where $U_{p}$ is the 
pondermotive potential discussed in Appendix III. Thus the maximum kinetic energy of the returning electron increases as the square of the wavelength increases [76]. Moreover, rescattering ionization will exhibit a threshold at the irradiance for which $3.17 U_{p}$ is equal to the ionization potential $E$. Table 7.5.1 lists the measured 0.1 ion appearance irradiances for linear polarization for the doubly ionized charge states and the nonsequential threshold irradiances, $I_{n s}^{\text {thr }}$, calculated by setting the ionization potential for the singly ionized charge state equal to

$$
\begin{aligned}
E(\mathrm{eV}) & =3.17 U_{p} \\
& =(3.17) 9.33 \times 10^{-14} I_{n s}^{t h r}\left(\mathrm{~W} / \mathrm{cm}^{2}\right) \lambda^{2}(\mu \mathrm{m})
\end{aligned}
$$

The 0.1 ion level for the appearance was chosen because the nonsequential part

\begin{tabular}{cccc}
\hline \hline Species & Wavelength & $I^{\text {opp }}\left(\mathrm{W} / \mathrm{cm}^{2}\right)$ & $I_{\mathrm{ns}}^{\mathrm{thr}}\left(\mathrm{W} / \mathrm{cm}^{2}\right)$ \\
\hline $\mathrm{He}^{+2}$ & $614 \mathrm{~nm}$ & $1.3 \times 10^{15}$ & $4.88 \times 10^{14}$ \\
$\mathrm{Ne}^{+2}$ & $614 \mathrm{~nm}$ & $8.5 \times 10^{14}$ & $3.67 \times 10^{14}$ \\
$\mathrm{He}^{+2}$ & $800 \mathrm{~nm}$ & $7.0 \times 10^{14}$ & $2.87 \times 10^{14}$ \\
$\mathrm{Ne}^{+2}$ & $800 \mathrm{~nm}$ & $6.0 \times 10^{14}$ & $2.16 \times 10^{14}$ \\
$\mathrm{Ar}^{+2}$ & $800 \mathrm{~nm}$ & $1.3 \times 10^{14}$ & $8.3 \times 10^{13}$ \\
\hline \hline
\end{tabular}

Table 7.5.1 The measured 0.1 ion appearance irradiances for linear polarization for the doubly ionized charge states and the corresponding calculated nonsequential threshold irradiances. 
of the 614-nm data begins to saturate below the 1 ion level. Where the $800-\mathrm{nm}$ data does not extend down to the 0.1 ion level, the curves were extrapolated. While the comparison between $I_{n s}^{\text {thr }}$ and $I^{\text {app }}$ is not rigorous, the nonsequential thresholds are all below the measured appearance irradiances by factors of 1.5 to 2.8. There is clearly no anomalous nonsequential ionization below the threshold of the Corkum model. Thus the rescattering process is possible for all the species that exhibited enhanced double ionization.

For a given species the wavelength dependence of the enhancements can also be compared by scaling the data so that the singly ionized charge states agree. Theoretically the scaling should only involve the difference in focal volume. This

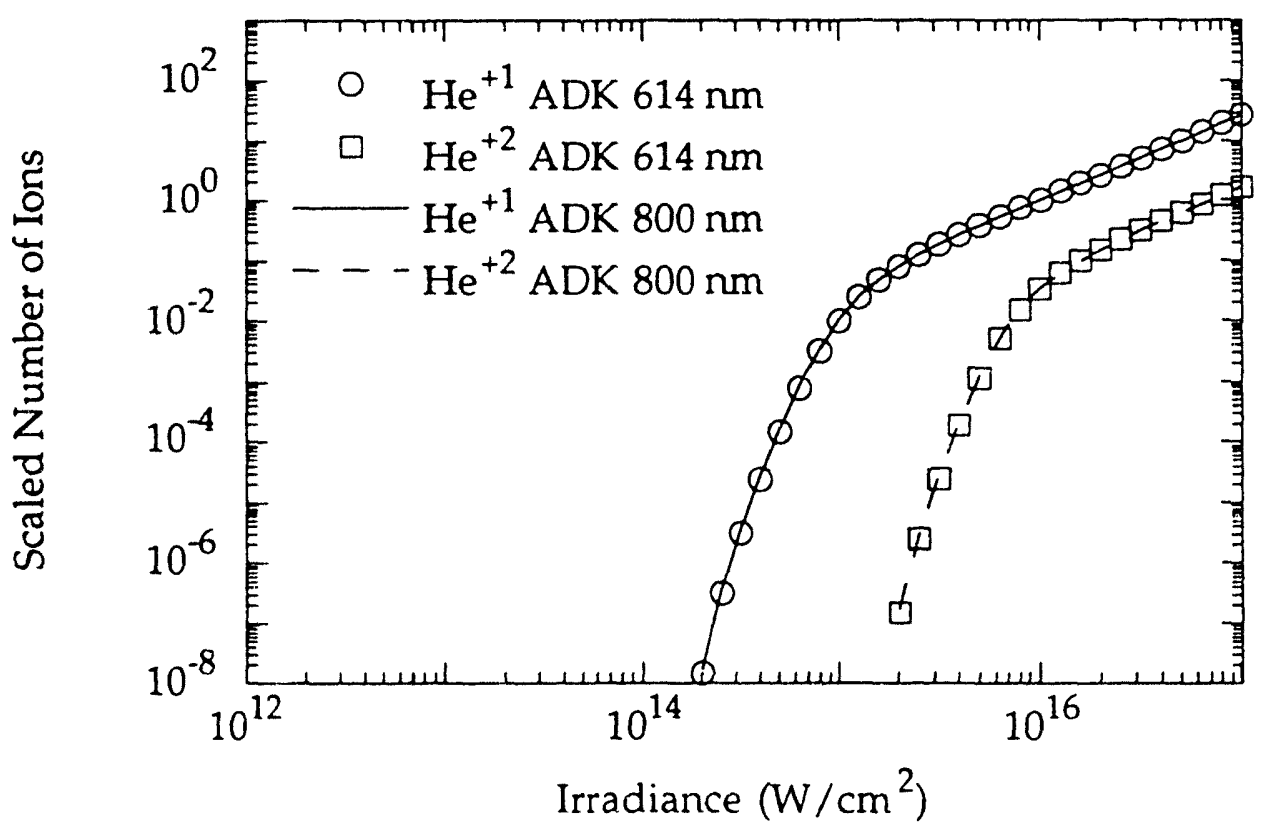

Figure 7.5.1 The ADK He ion yields for $614 \mathrm{~nm}$ and $800 \mathrm{~nm}$ scaled to remove the differences in characteristic focal volume. The theory is for linear polarization. 
is shown in Figure 7.5.1. For linear polarization the figure shows the ADK model He ion yields for $614 \mathrm{~nm}$ and $800 \mathrm{~nm}$ scaled to remove the differences in characteristic focal volume ( $V_{0}$ Equation 3.4 .2$)$. As noted previously all the data in this dissertation is already scaled to a target pressure of $1.0 \times 10^{-3} \mathrm{~Pa}$. Without differences in focal volume or density, the theoretical tunneling yields are obviously independent of wavelength. Scaling to the singly ionized charge states will also remove any systematic differences such as uncertainty in peak irradiance, in focal volume or in density between the $614-\mathrm{nm}$ and $800-\mathrm{nm}$ measurements. Figures 7.5.2 and 7.5.3 show the scaled yields for $\mathrm{He}$ and $\mathrm{Ne}$, respectively, at $614 \mathrm{~nm}$ and $800 \mathrm{~nm}$ with linear polarization. The scaled yields that the nonsequential part of the helium yield has increased approximately a

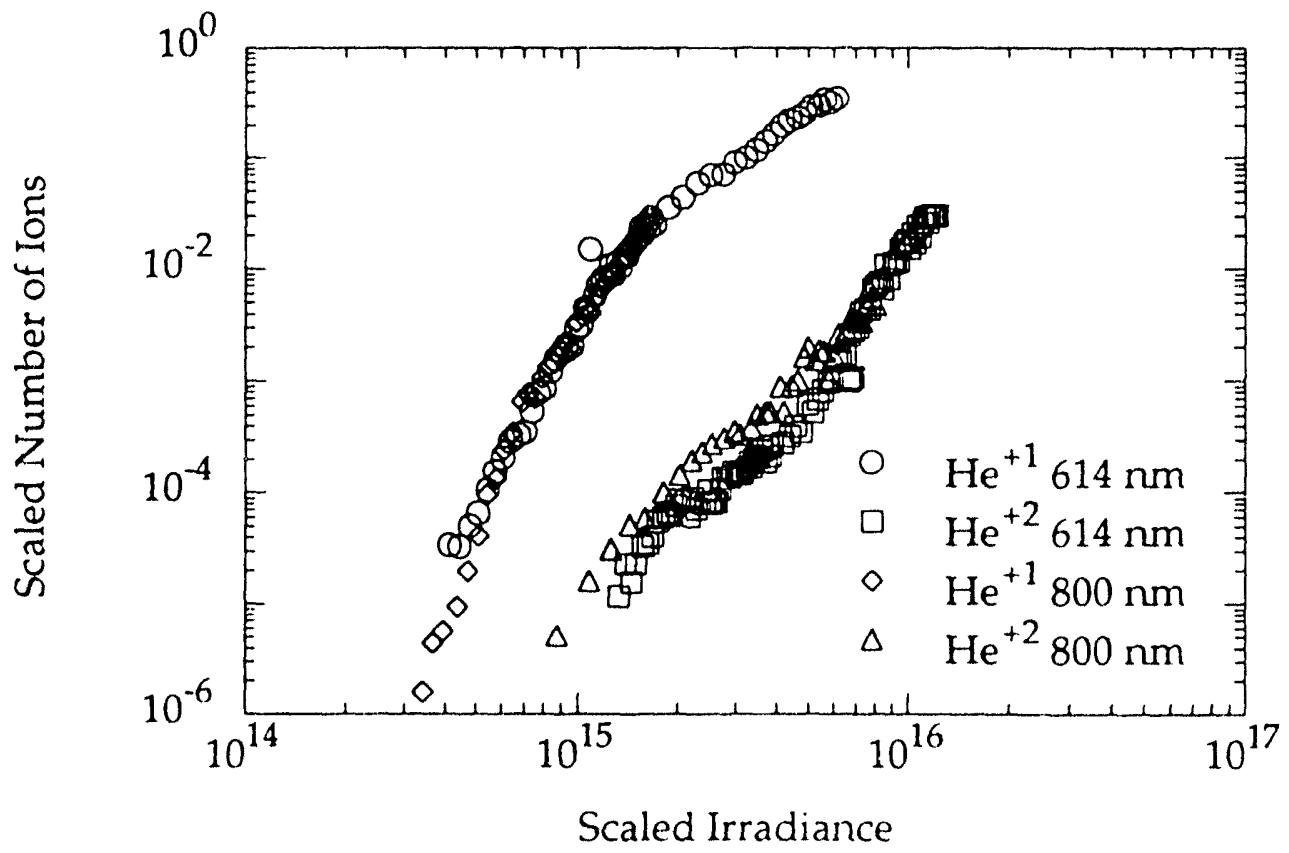

Figure 7.5.2 The helium ion yields for linear polarization at $614 \mathrm{~nm}$ and $800 \mathrm{~nm}$ scaled to the singly ionized charge states. 
factor of two as the wavelength was increased from $614 \mathrm{~nm}$ to $800 \mathrm{~nm}$. Such an increase is expected for the rescattering model because the rescattering energy increases with $\lambda^{2}$, and the cross section increases in the energy range of interest. Also the appearance irradiance at $800 \mathrm{~nm}$ is lower than the appearance irradiance at $614 \mathrm{~nm}$. This decrease is also expected for the scattering model as is seen in Equation (7.5.1) where the nonsequential irradiance threshold decreases as $\lambda^{2}$. The nonsequential part of the neon ion yield is unchanged to within the accuracy

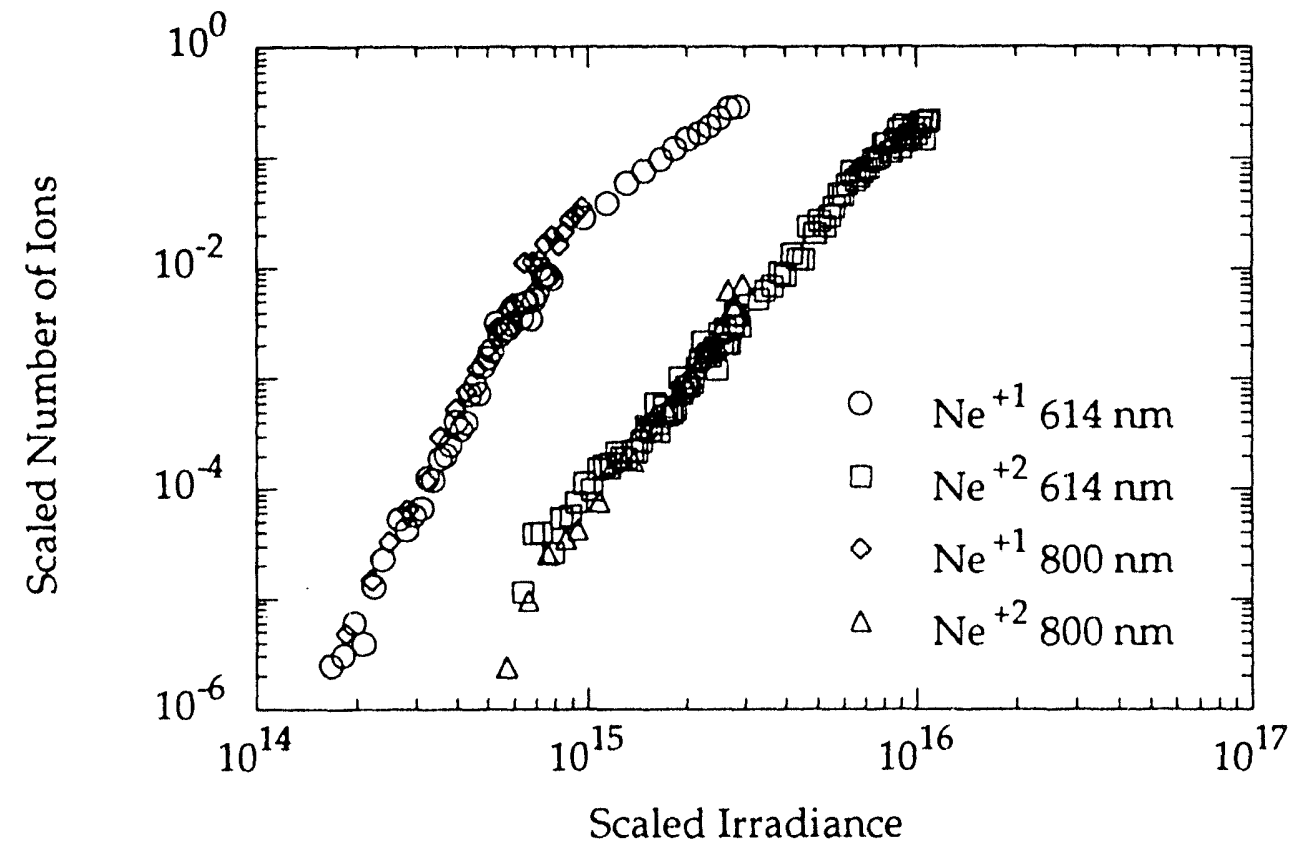

Figure 7.5.3 The neon ion yields for $614 \mathrm{~nm}$ and $800 \mathrm{~nm}$ scaled to the singly ionized charge states.

of the comparison. The wavelength scaling here is a mystery because effects similar to those for He are predicted. The wavelength comparison for helium is, however, cleaner than in neon. Energetically no other pathways for ionization 
exist for helium while other channels for ionization do exist for neon. The actual scaling of the rescattering model with wavelength requires knowledge of the

$$
\begin{aligned}
& \mathrm{He}^{+}+e \rightarrow \mathrm{He}^{+2}+2 e \text { and } \\
& \mathrm{Ne}^{+}+e \rightarrow \mathrm{Ne}^{+2}+2 e
\end{aligned}
$$

collisional ionization cross-sections for atoms under the influence of strong laser fields in addition to scaling of the energy of the returning electron. These crosssections are currently unknown. Based purely on the wavelength scaling, however, the nonsequential parts of the yields should increase by a small factor. The fact that the enhancements are similar for 614 and $800 \mathrm{~nm}$ in neon may indicate the action of other processes.

The wavelength scaling of the appearance irradiances for sequential ionization is also useful. Figure 7.5.4 shows the measured appearance irradiances for $\mathrm{He}^{+1}$ and $\mathrm{Ne}^{+1}$ with linear and circular polarization and for $\mathrm{He}^{+2}$ and $\mathrm{Ne}^{+2}$ for linear polarization at $800 \mathrm{~nm}$ plotted against their respective appearance irradiances at $614 \mathrm{~nm}$. The line plotted through the data indicates a linear relationship. In the figure the relationship of the data for the two wavelengths is linear. This indicates that the relative appearance irradiances are independent of the wavelength. For tunneling ionization the appearance irradiances should be independent of the laser wavelength. For multiphoton ionization, however, the relative appearance irradiances are expected to be wavelength dependent due to the different photon energies. The photon energy at $614 \mathrm{~nm}$ is $2.02 \mathrm{eV}$ while the photon energy at $800 \mathrm{~nm}$ is $1.55 \mathrm{eV}$. Thus producing $\mathrm{He}^{+2}$ from $\mathrm{He}^{+1}$ requires 27 photons at $614 \mathrm{~nm}$ and 35 photons at $800 \mathrm{~nm}$. Since the ion yields for MPI obey an irradiance to the number of photons power law, changing the wavelength (number of photons required) will alter the relative appearance irradiances. Thus 


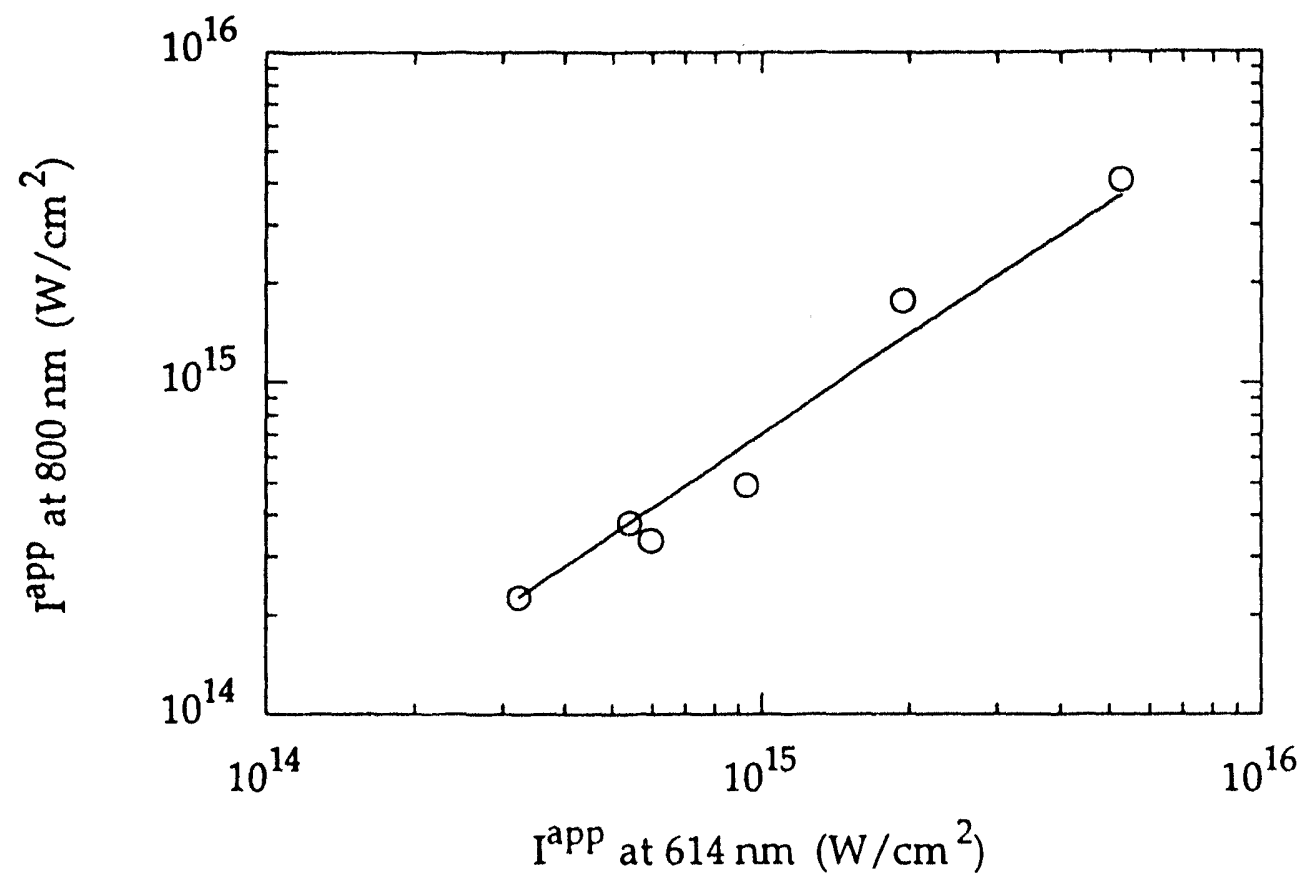

Figure 7.5.4 Comparison of the 614 and $800 \mathrm{~nm}$ single ion appearance irradiances for helium and neon. The data exclude the doubly charged states for linear polarization since those states exhibit enhanced double ionization. The line is placed to indicate a linear relationship between the data.

the independence of the relative appearance irradiances strengthens the assertion that the ionization occurs via tunneling.

\subsection{Summary}

For $130-\mathrm{fs}$, circularly polarized, $800-\mathrm{nm}$ pulses, the singly and doubly ionized ion yields of helium, neon and argon agree with the predictions of sequential ADK theory. For linearly polarized pulses, the ion yields exhibit the same type of direct ionization component that was observed for linearly polarized 614-nm pulses. Measurements for intermediate polarizations show that the enhancement 
decreases by two orders of magnitude as the ellipticity increases from 0 to 0.3 . The strong ellipticity dependence of the enhancements is consistent with the observations at $614 \mathrm{~nm}$ and with the predictions of the rescattering model for nonsequential ionization. In addition this strong ellipticity dependence has been confirmed by Walker et al.[66] and Kondo et al.[77] in helium and by Dietrich et al.[71] in neon. For linear polarization Kondo et al. have also shown that the nonsequential ionization feature seen in ${ }^{4} \mathrm{He}$ using $745 \mathrm{~nm}$ light is not observed in ${ }^{3} \mathrm{He}$ at $248 \mathrm{~nm}$. Although the pulse width at $248 \mathrm{~nm}$ was $440 \mathrm{fs}$ they attribute this result to the quadratic reduction of the "re-encounter" energy (relevant to the e-2e scattering) below the $54 \mathrm{eV}$ cross section threshold in helium. Our data and these other recent observations are consistent with Corkum's semi-classical description. Temporal scaling, however, was not investigated in this dissertation, and the absence of a direct ionization feature in ${ }^{3} \mathrm{He}$ using 1.5 ps pulses at 1053 $\mathrm{nm}[78]$ is still a puzzle in that it appears consistent with a shake-off picture. 


\section{CHAPTER 8-THE SPECIES DEPENDENCE OF SEQUENTIAL IONIZATION}

\subsection{Introduction}

As noted in Section 3.7 the major dependence of the appearance irradiance is a cubic dependence on the ionization potential. A weak density dependence was mentioned in Chapter 3. Prior to this work, Augst et al. reported an additional weak species dependence of the appearance irradiance for sequential ionization experiments with linearly polarized 1-ps pulses at $1 \mu \mathrm{m}[7]$. Gibson et al. also reported species dependence of the appearance irradiance for 500 -fs pulses at 248 $\mathrm{nm}[10]$. The nonsequential ionization observed in this dissertation shows that appearance irradiance measurements for ionization by linearly polarized 120 -fs pulses will be strongly influenced by nonsequential ionization for doubly ionized charge states. For circularly polarized pulses, however, nonsequential ionization-or for that matter resonantly enhanced ionization-is greatly reduced, and appearance irradiance measurements exhibit the dependences characteristic of the sequential ionization.

\subsection{Appearance Irradiances at $800 \mathrm{~nm}$ with Circular Polarization}

Figure 8.2.1 shows the measured single ion appearance irradiances for $\mathrm{He}, \mathrm{Ne}$ and Ar versus the ionization potential of the previous charge state for each species. The singly and doubly charged ion appearance irradiances are given. Since the higher charge states have higher ionization potential the doubly charge states are to the right of the singly charged states. The data for each species has been fitted by a power law curve. The powers in ionization potential range from 2.5 to 2.8. The He curve lies slightly higher than the Ne curve which is higher than the Ar curve. The ordering of this species dependence agrees with the 
previous measurements at $1 \mathrm{ps}$ for which the species of higher atomic number also appeared at lower irradiances $[7,10]$. Furthermore this confirms that any species dependence to sequential ionization is a weak dependence.

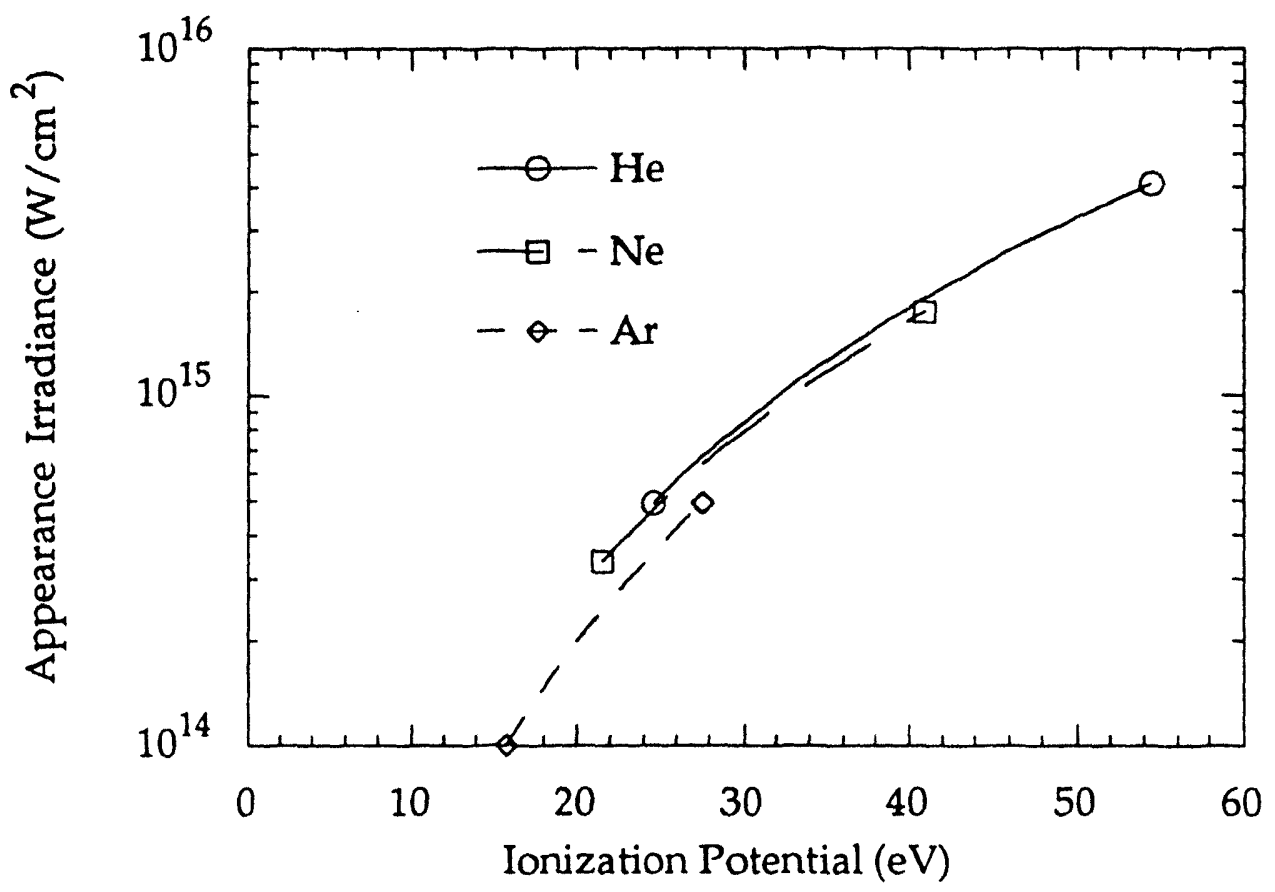

Figure 8.2.1 The single ion appearance irradiance as a function of ionization potential for singly and doubly charged states of $\mathrm{He}, \mathrm{Ne}$ and Ar produced by circularly polarized pulses at $800 \mathrm{~nm}$. The lines connecting the data are power law fits.

\subsection{Discussion of Barrier Suppression Ionization Description}

Augst explained the dependence of the appearance irradiance in terms of the ionization potential, $\mathrm{E}$, and the ionic charge of the species after ionization, $Z$, using the Barrier Suppression Ionization or BSI description that was mentioned briefly in Chapter 2. In the BSI description the system is treated classically in 1dimension. In atomic units the potential is then 


$$
V(x)=-\frac{Z}{|x|}-F_{0} x
$$

where $x$ is the distance from the nucleus. $F_{0}$ is the magnitude of the applied electric field. The system is solved trivially by setting $V\left(x_{\max }\right)=E$ where $x_{\max }$ is determined from

$$
\frac{\partial V}{\partial x}\left(x_{\max }\right)=0
$$

This gives the threshold static field above which all atoms ionize

$$
F_{0}^{\text {crit }}=\frac{E^{2}}{4 Z}
$$

This critical field is not directly related to the threshold fields defined in Chapter 2. The threshold field is defined as the field at which the probability of being in a given state $k$ reaches 0.01 . Once the critical field is reached the probability of ionization is 1 . In the notation of Chapter 2 where $E_{k}$ is twice the ionization potential of a species in charge state $k$ the critical field for production of charge state $k+1$ is

$$
F_{k+1}^{\text {cit }}=\frac{E_{k}^{2}}{16 Z_{k+1}} \text {. }
$$

where $Z_{k+1}$ is the ionic charge of the $k+1$ charge state. The critical irradiance is then obtained from Equation (2.7.6)

$$
I_{k+1}^{c r t}=\frac{1}{(2048) \pi \alpha}\left(1+\varepsilon^{2}\right) \frac{E_{k}^{4}}{Z_{k+1}^{2}}
$$

This BSI form for the critical irradiance is obviously flawed because it is entirely independent of the laser pulse width and does not provide an irradiance or field dependent ionization rate. It is possible to calculate the number of ions produced 
in closed form because in a laser focus the critical irradiances clearly define shells of charge in the spatial profile of the laser. Thus

$$
N_{k+1}=\rho\left[V\left(I_{k+1}^{c r i t}\right)-V\left(I_{k+2}^{c r i t}\right)\right]
$$

where $V(I)$ is the volume defined in Equation (3.4.1) for a peak irradiance $I_{0}$. In the BSI description the prediction for the number of ions is independent of the laser pulse width-and even the temporal profile-while the predictions for the more sophisticated models given in Chapter 2 all show a linear dependence on the pulse width for hyperbolic-secant pulses.

Consider the BSI explanation of the species dependence in terms of the effective principle quantum number of charge state $k$

$$
n^{*}=\frac{Z}{\sqrt{2 E}}=\frac{Z_{k+1}}{\sqrt{E_{k}}}
$$

The critical irradiance may be cast in terms of this effective principle quantum number

$$
I_{k+1}^{c n t} \propto \frac{E_{k}{ }^{3}}{\left(n^{*}\right)^{2}} .
$$

In other words, the species dependence of $I_{k+1}^{c n t}$ for a given ionization potential is a simple $\frac{1}{\left(n^{*}\right)^{2}}$ factor.

\subsection{Species Dependence of the ADK Model}

Using the ADK model provides a clearer picture of the species dependence of the appearance irradiance. Following the techniques of Chapter 3, for circular polarization the ADK ion appearance irradiance is 


$$
I_{k+1}^{a p p a r}=\frac{1}{9 \pi \alpha} E_{k}^{3} /\left[\ln \left[\omega_{0} \tilde{C}_{n l}{ }^{2} G_{l m} \frac{E_{k}}{2}\left(\frac{2 \tau}{176}\right)\left(\pi \rho V_{0}\right) / N_{a p}\right]\right]^{2}
$$

While the temporal pulse shape used to obtain Equation (8.4.1) is a squared hyperbolic-secant, this pulse shape approximates the Gaussian temporal profile (the actual profile of the $800-\mathrm{nm}$ laser pulses) well. The agreement of between calculations based on a hyperbolic secant profile and a Gaussian profile has been tested numerically and has proven to be excellent. Thus we can use Equation (8.4.1) to see that the species dependence arises through the quantum number dependence of the factor $\tilde{C}_{n !}{ }^{2} G_{l m}$ where $\tilde{C}_{n l}{ }^{2}$ and $G_{l m}$ are given by Equations (3.7.3) and (3.7.4). Figure 8.4.1 shows $I_{k+1}^{\text {appear }}$ plotted versus ionization potential for $n=1, . ., 5$. The other quantum numbers

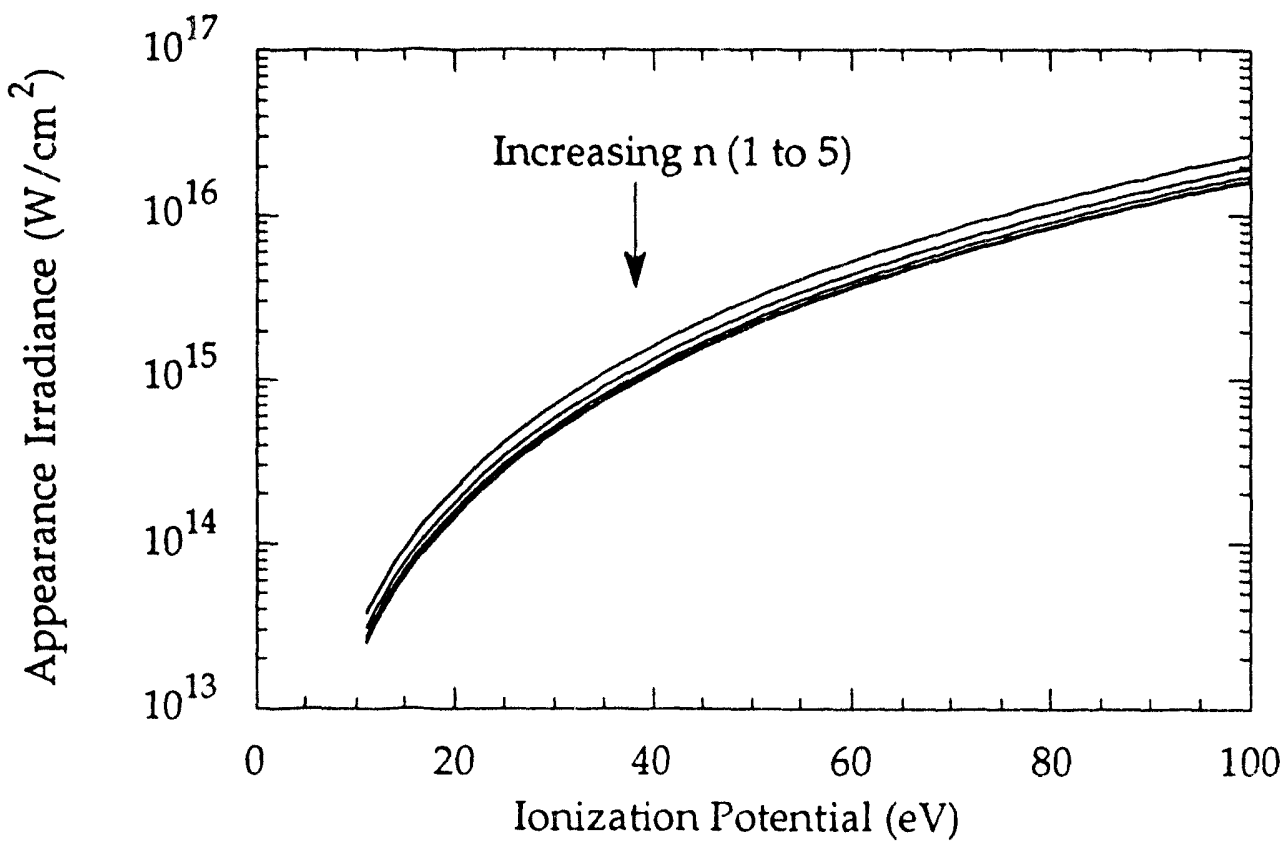

Figure 8.4.1 The appearance irradiance from the ADK model for circular polarization plotted versus ionization potential for $n=1$ to 5 . The other quantum numbers are set to $l=m=0$. The curves of smaller $\mathrm{n}$ values lie above those of larger $\mathrm{n}$ values. 
are set to $l=m=0$. The curves of smaller $n$ values lie above those of larger $n$ values. This ordering is characteristic of the data of this experiment and of Augst (Figure 2 of [7]) and Gibson (Figure 1a of [10]). Thus the species dependence of the appearance irradiance can be interpreted as principle quantum number dependence of the ionization rate coefficient.

Also using the prescription of replacing $n$ by $n^{*}$ and $l$ by $l^{*}$ in $\tilde{C}_{n l}{ }^{2}$, the ADK appearance irradiance can be shown to be a function of $n^{*}$,

$$
I_{k+1}^{a p p a r}=\frac{1}{9 \pi \alpha} E_{k}^{3} /\left[\ln \left[\omega_{0} \tilde{C}_{n \cdot \nu^{2}} G_{l m} \frac{E_{k}}{2}\left(\frac{2 \tau}{176}\right)\left(\pi \rho V_{0}\right) / N_{a p}\right]\right]^{2}
$$

Substituting the form of $\tilde{C}_{n} \nu^{2}$ in the appearance irradiance it can then be shown that

$$
I_{k+1}^{a p p e a r} \propto \frac{E_{k}^{3}}{f\left(n^{*}\right)}
$$

where $f\left(n^{*}\right)$ is a weak function of $n^{*}$. Figure 8.4.2 shows the calculated appearance irradiances for the ADK model for circular polarization for the two charge states of helium and the first four charge states of neon and argon. The calculations are for the conditions of the $800-\mathrm{nm}$ experiments. The agreement with the data shown in Figure 8.2.1 is excellent. The powers in the ionization potential range from 2.3 to 2.7 in agreement with the powers fitted to the data. The accuracy of the power fits is obviously limited by the small number of points fitted in both cases. The $n^{*}$ for the neutral and singly ionized ions of helium, neon and argon are shown in Table 8.4.1. Note that the accuracy of irradiance measurements that are based on cubic fits to the ionization potentials of the measured species is not greatly affected by the deviation from cubic caused by $f\left(n^{*}\right)$. 


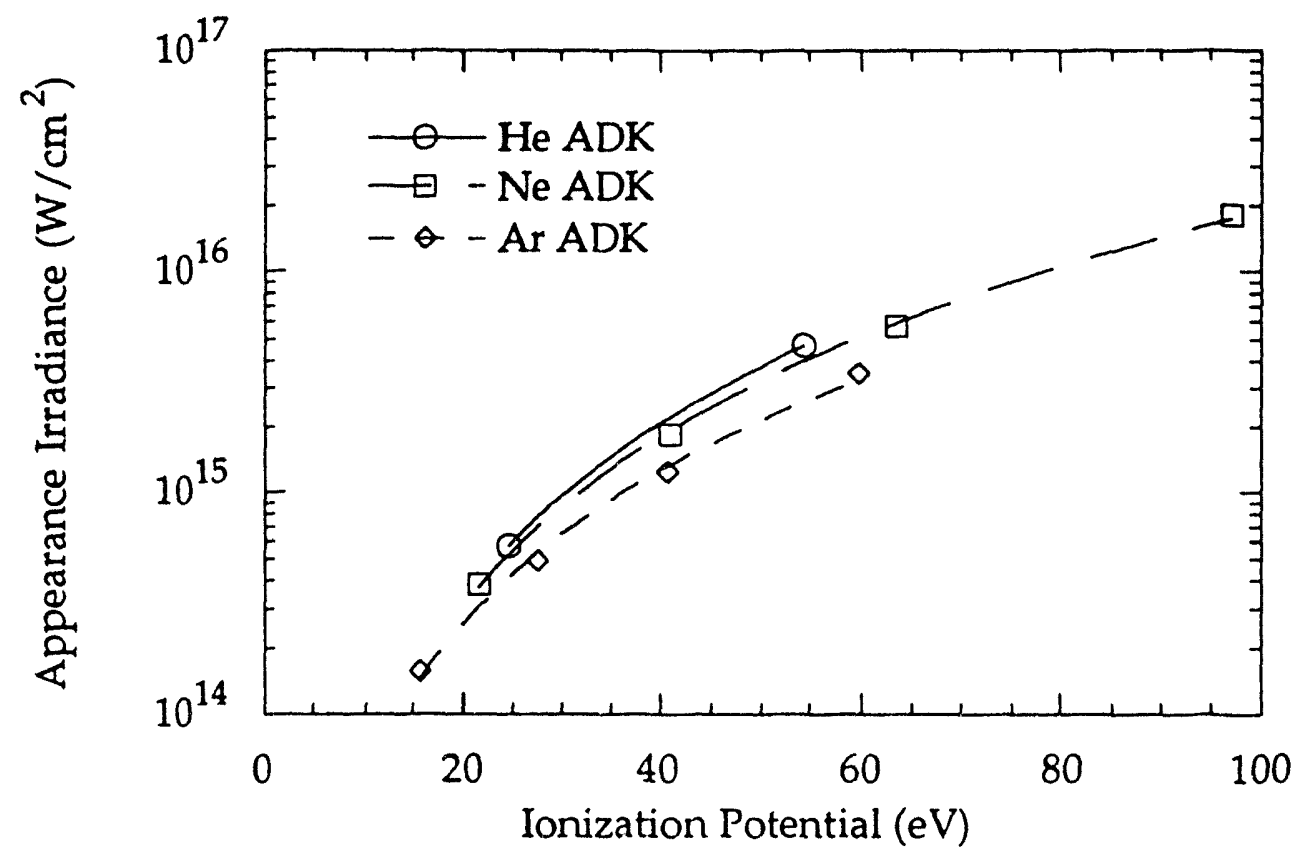

Figure 8.4.2 The ADK model appearance irradiances as a function of ionization potential for $\mathrm{He}, \mathrm{Ne}$ and Ar produced by circularly polarized pulses at $800 \mathrm{~nm}$. The singly and doubly charged states are shown. The triply and quadruply charged states are also shown for $\mathrm{Ne}$ and $\mathrm{Ar}$. The lines connecting the data are power law fits.

\begin{tabular}{cccc}
\hline \hline Species & $n^{*}$ & Species & $n^{*}$ \\
\hline $\mathrm{He}$ & 0.743 & $\mathrm{He}^{+1}$ & 1.000 \\
$\mathrm{Ne}$ & 0.794 & $\mathrm{Ne}^{+1}$ & 1.153 \\
$\mathrm{Ar}$ & 0.929 & $\mathrm{Ar}^{+1}$ & 1.403 \\
\hline
\end{tabular}

Table 8.4.1 The effective principle quantum numbers for the neutral and singly ionized ions of helium, neon and argon. 
Figure 8.4.3 shows the measured appearance irradiances with d cubic power fit. Singly and doubly charged states of $\mathrm{He}, \mathrm{Ne}$ and $\mathrm{Ar}$ ionized by circularly polarized $800-\mathrm{nm}$ pulses are shown. The agreement is good. The best power fit is ionization potential to the 2.88 power with a .999 confidence level.

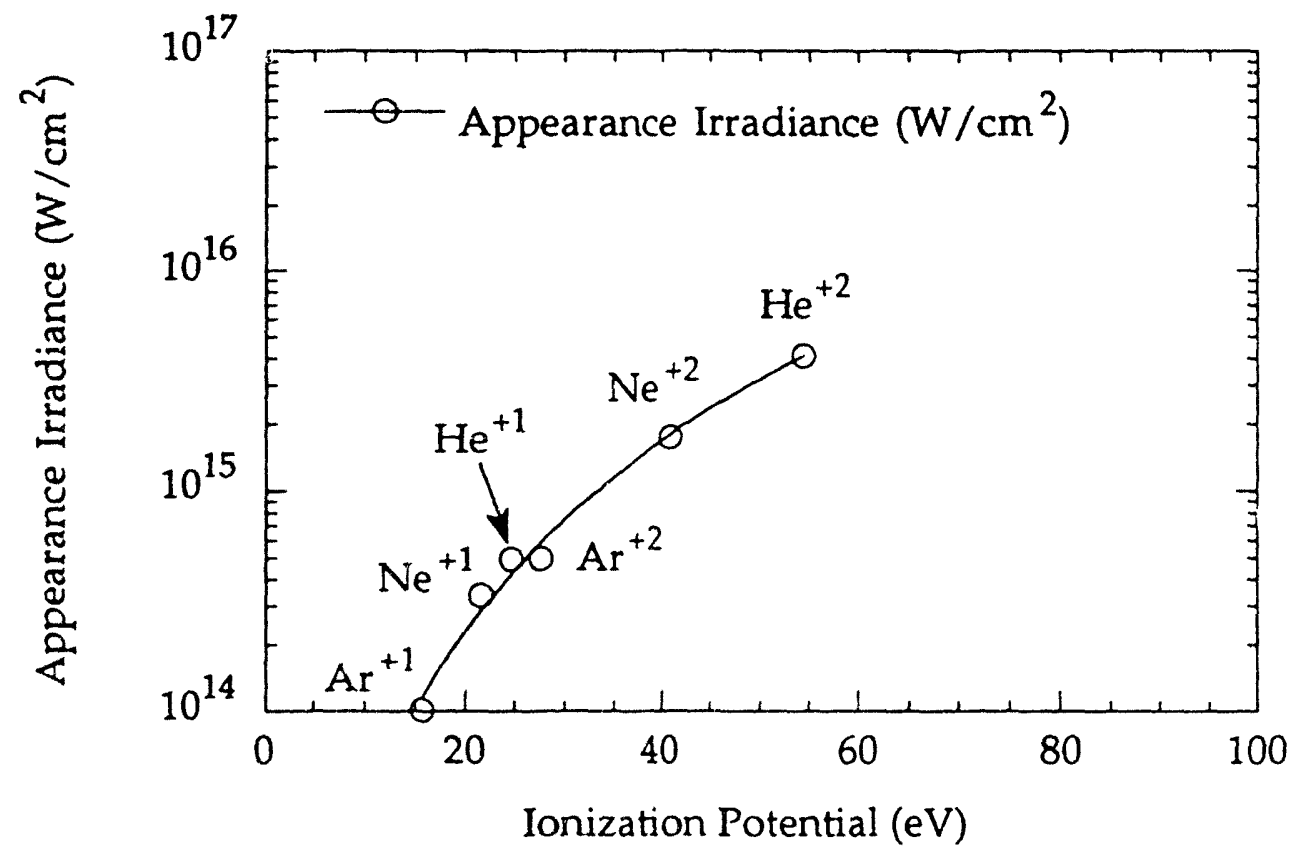

Figure 8.4.3 The measured appearance irradiances with a cubic power fit in the ionization potential. The data are for circular polarization at $800 \mathrm{~nm}$.

\subsection{Summary}

Using circular polarization to eliminate nonsequential effects allows unperturbed measurement of the appearance irradiances. In the data for circularly polarized $800-\mathrm{nm}$ pulses, a species dependence is observed that is consistent with that previously observed at longer pulse widths. An analytic solution for the ADK model attributes the species dependence to the principle quantum number of the 
134

ionizing species. Using the effective principle quantum number $n^{*}$, shows a generally cubic dependence on the ionization potential modified by a weak function of $n^{*}$. This form of the species dependence agrees qualitatively with the form obtained using classical estimates such as the BSI description. But the analytic solutions for the ADK model are able to accurately predict measured appearance irradiance in addition to demonstrating a weak species dependence in terms of $n^{*}$. Also since the enhancements disappear for circular polarization, any reliable scheme to monitor appearance irradiance should employ circular polarization only. 


\section{CHAPTER 9- CONCLUSIONS}

In summary, this dissertation research produced the first evidence of nonsequential optical field ionization by ultrashort laser pulses at $614 \mathrm{~nm}$ and $800 \mathrm{~nm}$. The evidence appeared as enhancements in the number of $\mathrm{He}^{+2}, \mathrm{Ne}^{+2}$ and $\mathrm{Ar}^{+2}$ produced in the focuses of the lasers. These enhancements were shown to be inconsistent with sequential tunneling ionization and cannot be explained by contaminant ions or resonance effects. Two direct nonsequential mechanisms were considered as causes for the enhancements: a shake-off mechanism and a rescattering mechanism. Based on the predictions of the mechanisms further measurements were made using alternate polarizations that supported the rescattering mechanism by showing a rapid decrease in the enhancement as the polarization goes from linear to circular. Also in helium-where the nonsequential effect is most pronounced-a clear wavelength scaling is seen as predicted by the rescattering model. Because the rescattering model involves simplistic quasiclassical calculations further theoretical investigation on the quantum mechanical nature of such rescattering is necessary along with solution of the full two-electron problem[79]. Further experiments measuring the electron coincidence and energy distributions are also needed to strengthen the picture that has arisen from the ion yield and harmonic generation experiments. Experiments investigating the pulse width dependence of the effect in a controlled way are also warranted since the pulse width dependence of nonsequential effects is not well known. Also the yields for sequential tunneling should increase linearly with the pulse width.

The polarization dependence of the nonsequential ionization has a major impact on the use of field ionization yields to monitor the peak irradiance of 
pulses with pulse widths on the order of $100 \mathrm{fs}$. Typically ideas for monitoring irradiance have been based on linear polarization. In practice linear polarization is unsuited for irradiance measurements due to the presence of enhancements for doubly and possibly more highly charged states. Thus irradiance monitoring using only circular polarization is more reliable and viable.

Several aspects of ionization have also been investigated that indicate that for sequential ionization the ionization occurs through optical field or tunneling ionization. Using circular polarization to eliminate the enhanced ionization has shown that

$$
I^{a p p} \propto \frac{E_{k}^{3}}{f\left(n^{*}\right)}
$$

where $f\left(n^{*}\right)$ is a weak function of $n^{*}$. This predominantly cubic dependence of the appearance irradiance on ionization potential - and weak dependence on the species through $n^{*}$-agrees with the 1-D BSI description and previous data but is more rigorously based on 3-D tunneling using the ADK model, which provides actual ionization rates. The relative appearance irradiances for sequential ionization are also largely wavelength independent as expected for tunneling but not for multiphoton ionization. In addition, comparisons for linear and circular polarization for singly ionized charge states show the field scaling predicted by tunneling ionization (i.e. appearance irradiances differing by a factor of two).

In general, this work has mapped strong optical field ionization in the regime bounded by $100 \mathrm{fs}$ dynamics and fields on the order of $1 \mathrm{au}$. It has validated tunneling ionization as the major mechanism for such ionization with rescattering effects being important in multiple ionization. This work has prompted a renewed interest in strong field ionization physics and the dynamics 
of semi-classical optical field effects. Knowledge of the mechanisms is critical for future experiments on tunnel-ionized plasmas, $X$-ray lasers, pulse propagation and ionization front propagation for which the atomic nature of the ionized plasma is important. 


\section{REFERENCES}

[1] J. R. Oppenheimer, Phys. Rev. 13, 66-81 (1928).

[2] T. W. Ducas, M. G. Littman, R. R. Freeman and D. Kleppner, Phys. Rev. Lett. 35, 366-369 (1975).

[3] H. B. van Linden van den Heuvall, R. Kachru, N. H. Tran and T. F. Gallagher, Phys. Rev. Lett. 53, 1901-1904 (1984).

[4] B. E. Sauer, S. Yoakum, L. Moorman, P. M. Koch, D. Richards and P. A. Dando, Phys. Rev. Lett. 68, 468-471 (1992).

[5] B. W. Boreham and B. Luther-Davies, J. Appl. Phys. 50, 2533-2538 (1979).

[6] B. W. Boreham and J. L. Hughes, Sov. Phys. JETP 53, 252-259 (1981).

[7] S. Augst, D. Strickland, D. D. Meyerhofer, S. L. Chin and J. H. Eberly, Phys. Rev. Lett 63, 2212-2215 (1989).

[8] S. Augst, D. D. Meyerhofer, D. Strickland and S. L. Chin, J. Opt. Soc. Am. B 8 , 858-867 (1991).

[9] S. J. Augst, "Tunneling ionization of noble gas atoms using a high intensity laser at $1 \mu \mathrm{m}$ wavelength," Ph.D. Dissertation at University of Rochester (1991)

[10] G. Gibson, T. S. Luk and C. K. Rhodes, Phys. Rev. A 41, 5049-5052 (1990).

[11] K. G. H. Baldwin and B. W. Boreham, J. Appl. Phys. 52, 2627-2633 (1981).

[12] M. D. Perry, O. L. Landen, A. Szöke and E. M. Cambell, Phys. Rev. A 37, 747-760 (1988).

[13] M. D. Perry, "High-Order Multiphoton Ionization of Noble Gases," Ph.D. Thesis at University of California at Davis (1987)

[14] S. L. Chin, C. Rolland, P. B. Corkum and P. Kelly, Phys. Rev. Lett. 61, 153156 (1988).

[15] A. l'Huillier, L. A. Lompré, G. Mainfray and C. Manus, J. Phys. B: At. Mol. Phys. 16, 1363-1381 (1983). 
[16] A. l'Huillier, L. A. Lompré, G. Mainfray and C. Manus, Phys. Rev. A 27, 2503-2512 (1983).

[17] D. N. Fittinghoff, P. R. Bolton, B. Chang and K. C. Kulander, Phys. Rev. Lett. $62,2642-2645$ (1992).

[18] D. N. Fittinghoff, P. R. Bolton, B. Chang and K. C. Kulander, Proceedings of SPIE-The International Society for Optical Engineering 1860, SPIE Vol. 1860, pp. 209-215 (1993)

[19] D. N. Fittinghoff, P. R. Bolton, B. Chang and K. Kulander, in press Phys. Rev. A Brief Reports (1993).

[20] M. Weissbluth, Atoms and Molecules, Academic Press, Inc., San Diego (1978).

[21] J. J. Sakurai, Advanced Quantum Mechanics, Addison-Wesley Publishing Company, Reading, Massachusetts (1967).

[22] F. H. M. Faisal, Theory of Multiphoton Ionization, Plenum, New York (1987).

[23] N. B. Delone and V. P. Krainov, Atoms in Strong Light Fields, SpringerVerlag, Berlin (1985).

[24] L. D. Landau and E. M. Lifshitz, Quantum Mechanics Non-Relativistic Theory, Pergammon, Oxford (1965).

[25] L. V. Keldysh, 20, 1307-1314 (1965).

[26] F. A. Ilkov, J. E. Decker and S. L. Chin, J. Phys. B 25, (1992).

[27] A. M. Perelomov, V. S. Popov and M. V. Terent'ev, Sov. Phys. JETP 23, 924934 (1966).

[28] A. M. Perelomov, V. S. Popov and M. V. Terent'ev, Sov. Phys. JETP 24, 207217 (1967).

[29] A. M. Perelomov, V. S. Popov and M. V. Terent'ev, Sov. Phys. JETP 25, 336343 (1967).

[30] B. M. Smirnov and M. I. Chibisov, Sov. Phys. JETP 22, 585-592 (1966). 
[31] M. V. Ammosov, N. B. Delone and V. P. Krainov, Sov. Phys. JETP 64, 11911194 (1986).

[32] M. V. Ammosov, N. B. Delone and V. P. Krainov, personal communication (1991).

[33] K. J. Schafer, J. L. Krause and K. C. Kulander, "Nonlinear effects in electron and photon emission from atoms in intense laser fields," Lawrence Livermore National Laboratory Report, JC-107014 (1991)

[34] K. C. Kulander, K. J. Schafer and J. L. Krause, Time-dependent studies of multiphoton processes in, "Atoms in Intense Radiation Fields", Edited by M. Gavrila, Academic Press, New York (1992)

[35] K. C. Kulander and K. J. Schafer, "Phase effects in two-color multiphoton processes," Lawrence Livermore National Laboratory Report, JC-108407 (1991)

[36] K. C. Kulander, J. Schafer and J. L. Krause, "Time-dependent numerical studies of multiphoton processes," Lawrence Livermore National Laboratory Report, UCRL-JC-106699 (1991)

[37] J. L. Krause, K. J. Schafer and K. C. Kulander, Phys. Rev. Lett. 68, 3535-3538 (1992).

[38] D. R. Nicholson, Introduction to Plasma Theory John Wiley \& Sons, New York (1983).

[39] P. Lambropoulos, Phys. Rev. Lett. 55, 2141-2144 (1985).

[40] F. Yergeau, S. L. Chin and P. Lavigne, J. Phys. B: At. Mol. Phys. 20, 723-739 (1987).

[41] B. Chang, P. R. Bolton and D. N. Fittinghoff, Phys. Rev. A 47, 4193-4203 (1993).

[42] M. Abramowitz and I. A. Stegun, Handbook of Mathematical Functions, Edited by Dover Publications, Inc., New York (1972) 
[43] I. S. Gradshteyn and I. M. Ryzhik, Table of Integrals, Series, and Products, Academic Press, Inc., New York (1980).

[44] P. Agostini, F. Fabre, G. Mainfray, G. Petite and N. K. Rahman, Phys. Rev. Lett. 42, 1127-1130 (1979).

[45] J. Diels, W. Dietel, J. J. Fontaine, W. Rudolph and B. Wilhelmi, J. Opt. Soc. Am B 2, 680-686 (1985).

[46] J. P. Gordon and R. L. Fork, Opt. Lett. 9, 153-155 (1984).

[47] H. A. Haus, IEEE J. of Quantum Electron. QE-11, 736-746 (1975).

[48] J. A. Valdmanis, R. L. Fork and J. P. Gordon, Opt. Lett. 10, 131-133 (1985).

[49] J. A. Valdmanis and R. L. Fork, IEEE J. Quantum Electron. QE-22, 112-118 (1986).

[50] D. S. Bethune, Appl. Opt. 20, 1897-1899 (1981).

[51] J. Janszky, G. Corradi and R. N. Gyuzalian, Opt. Comm. 23, 293-298 (1977).

[52] R. N. Gyuzalian, S. B. Sogomonian and Z. G. Horvath, Opt. Comm. 29, 239242 (1979).

[53] F. Salin, P. Georges, G. Roger and A. Brun, Appl. Opt. 26, 4528-4531 (1987).

[54] K. L. Sala, G. A. Kenney-Wallace and G. E. Hall, IEEE J. Quant. Electron. QE-16, 990-996 (1980).

[55] R. W. Boyd, Nonlinear Optics, Academic Press, Inc., San Diego (1992).

[56] W. C. Wiley and I. H. McLaren, Rec. Sci. Instr. 26, 1150-1157 (1955).

[57] M. V. Ammosov, F. A. Il'kov, M. G. Malakhov and C. K. Mukhtarov, J. Opt. Soc. Am. B 6, 1961-1963 (1989).

[58] R. D. Andersen and D. E. Page, Rev. Sci. Instr. 42, 371-372 (1971).

[59] E. H. Eberhardt, Appl. Opt. 18, 1418-1423 (1979).

[60] N. Koshida and N. Nagashima, Rev. Sci. Instrum. 54, 62-64 (1983).

[61] P. Lecomte and V. Perez-Mendez, IEEE Trans. Nuc. Sci. NS-25, 964-973 (1978). 
[62] D. Rogers and R. F. Malina, Rev. Sci. Instrum. 53, 1438-1441 (1982).

[63] J. G. Timothy, Rev. Sci. Instrum. 45, 834-837 (1974).

[64] Microchannel Plate Report, Chapter 1, Technische Informatie Post

Institute Voor Atoom En Molecuulfysica,

[65] P. B. Corkum, Phys. Rev. Lett. 71, 1994-1997 (1993).

[66] B. Walker, E. Mevel, B. Yang, P. Breger, J. P. Chambaret, A. Antonetti, L. F. DiMauro and P. Agostini, Phys. Rev. A Rapid Comm. 48, R894-R897 (1993).

[67] E. Mevel, P. Breger, R. Trainham, G. Petite, P. Agostini, A. Migus, J. P. Chambaret and A. Antonetti, Phys. Rev. Lett. 70, 406 (1993).

[68] U. Mohideen, M. H. Sher, W. K. Tom, G. D. Aumiller, O. R. Wood, R. R. Freeman, J. Bokor and P. Bucksbaum H., Phys. Rev. Lett. (submitted)

[69] R. Wehlitz, F. Heiser, O. Hemmers, B. Langer, A. Menzel and B. Becker, Phys. Rev. Lett. 67, 3764-3767 (1991).

[70] K. J. Schafer, B. Yang, L. F. DiMauro and K. C. Kulander, Phys. Rev. Lett. 70, 1599 (1993).

[71] P. Dietrich, N. H. Burnett, M. Ivanov and P. B. Corkum, to be published (1993).

[72] P. Defrance, F. Brouillard, W. Claeys and G. Van Wassenhove, J. Phys. B: At. Mol. Phys. 14, 103-110 (1981).

[73] M. J. Diserens, M. F. A. Harrison and A. C. H. Smith, J. Phys. B: At. Mol. Phys. 17, L621-L624 (1984).

[74] P. H. Bucksbaum, M. Bashkansky, R. R. Freemen, T. J. McIlrath and L. F. DiMauro, Phys. Rev. Lett. 56, 2590-2593 (1986).

[75] P. Corkum, personal communication (1993). The data has been scaled to account for the different focal volumes used in the calculation and the experiment. 
[76] H. Tawara and T. Kato, Atomic Data and Nuclear Data Tables 36, 167-353 (1987).

[77] K. Kondo, A. Sagisaka, T. Tamida, Y. Nabekawa and S. Watanabe, submitted to Phys. Rev. A Rapid. Comm. (1993).

[78] J. Peatross, B. Buerke and D. Meyerhofer, Phys. Rev. A 47, 1517-1519 (1993).

[79] B. Ritchie, Laser Physics 3, 355-358 (1993).

[80] M. Born and E. Wolf, Principles of Optics, Pergamon Press, Oxford (1975).

[81] T. W. B. Kibble, Phys. Rev. Lett. 16, 1054-1056 (1966).

[82] T. W. B. Kibble, Phys. Rev. 150, 1060-1069 (1966).

[83] R. R. Freeman, P. H. Bucksbaum, W. E. Cooke, G. Gibson, T. J. McIlrath and L. D. Van Woerkom, "Photoionization of atoms with ultra-short laser pulses," AT\&T Bell Laboratories Report, Work Project No. 311102-0205

File Case 38140

11122-910118-01 (1991)

[84] K. Miyamoto, Plasma Physics for Nuclear Fusion, Revised Edition, The MIT Press, Cambridge, Massachusetts (1989).

[85] P. Agostini, J. Kupersztych, L. A. Lompré, G. Petite and F. Yergeau, Phys. Rev. A 36, 4111-4114 (1987).

[86] P. H. Bucksbaum, R. R. Freeman, M. Bashansky and T. J. Mcllrath, J. Opt. Soc. Am. B 4, 760-764 (1987).

[87] R. R. Freeman, T. J. McIlrath, P. H. Bucksbaum and M. Bashansky, Phys. Rev. Lett. 57, 3156-3159 (1986).

[88] H. G. Muller, A. Tip and M. J. van der Wiel, J. Phys. B: At. Mol. Phys. 16, L679-L685 (1983).

[89] S. Zhu and W. Shen, J. Opt. Soc. Am. B 4, 739-742 (1987).

[90] R. P. Freeman, P. H. Bucksbaum, H. Milchberg, S. Darack, D. Schumacher and M. E. Geusic, Phys. Rev. Lett. 59, 1092-1095 (1987). 
[91] G. Petite, P. Agostini and F. Yergeau, J. Opt. Soc. Am B 4, 765-769 (1987).

[92] H. A. Bethe and E. E. Salpeter, Quantum Mechanics of One- and TwoElectron Atoms, Plenum Publishing Corporation, New York (1977). 


\section{APPENDIX I-THE STOKES PARAMETERS}

Following Born \& Wolf[80], for a plane wave the harmonic variation of the electric field may be represented by

$$
F=F_{x} \hat{e}_{x}+F_{y} \hat{e}_{y}
$$

where

$$
\left.\begin{array}{l}
F_{x}=a_{1} \cos \left(\omega t-k \cdot r+\delta_{1}\right) \\
F_{y}=a_{2} \cos \left(\omega t-k \cdot r+\delta_{2}\right)
\end{array}\right\}
$$

Eliminating the factor $\omega t-k \cdot r$ in Equations (A.I.2) yields

$$
\left(\frac{F_{x}}{a_{1}}\right)^{2}+\left(\frac{F_{y}}{a_{2}}\right)^{2}-2 \frac{F_{x}}{a_{1}} \frac{F_{y}}{a_{2}} \cos \delta=\sin ^{2} \delta
$$

where

$$
\delta=\delta_{2}-\delta_{1}
$$

The four Stokes parameters are defined

$$
\left.\begin{array}{l}
s_{0}=\left\langle a_{1}{ }^{2}\right\rangle+\left\langle a_{2}{ }^{2}\right\rangle, \\
s_{1}=\left\langle a_{1}{ }^{2}\right\rangle-\left\langle a_{2}{ }^{2}\right\rangle, \\
s_{2}=2\left\langle a_{1} a_{2} \cos \delta\right\rangle, \\
s_{3}=2\left\langle a_{1} a_{2} \sin \delta\right\rangle .
\end{array}\right\}
$$

Equation (A.I.3) describes the polarization ellipse. Let $\hat{e}_{\xi}$ and $\hat{e}_{\eta}$ be the direction of the major and minor axes of the ellipse, respectively. If $\psi(0 \leq \psi<\pi)$ is the angle between the $x$ and $\xi$ axes, the electric field components are then 


$$
\left.\begin{array}{l}
F_{\xi}=F_{x} \cos \psi+F_{y} \sin \psi, \\
F_{n}=-F_{x} \sin \psi+F_{y} \cos \psi .
\end{array}\right\}
$$

If $a$ and $b$ are the major and minor axes of the polarization ellipse, then the equation of the ellipse becomes

$$
\left.\begin{array}{l}
F_{\xi}=a \cos \left(\omega t-k \cdot r+\delta_{0}\right), \\
F_{\eta}= \pm b \sin \left(\omega t-k \cdot r+\delta_{0}\right) .
\end{array}\right\}
$$

where $\delta_{0}$ is a common phase factor. The angle $\chi(-\pi / 4 \leq \chi \leq \pi / 4)$ characterizes the ellipticity as given in Equation (2.7.1).

$$
\varepsilon=\tan \chi= \pm b / a
$$

In terms of the Stokes parameters

$$
\sin 2 \chi=\frac{s_{3}}{\sqrt{s_{1}{ }^{2}+s_{2}{ }^{2}+s_{3}{ }^{2}}}
$$

and

$$
\tan 2 \psi=\frac{s_{2}}{s_{1}}
$$

Experimentally I obtained the Stokes parameters by measuring the energy transmitted through a polarizer set at an angle $\theta$ with respect to the $x$-axis and a retardation $\zeta$. The Stokes parameters are related to the transmitted irradiances $I(\theta, \zeta)$ by

$$
\left.\begin{array}{l}
s_{0}=I\left(0^{\circ}, 0\right)+I\left(90^{\circ}, 0\right), \\
s_{1}=I\left(0^{\circ}, 0\right)-I\left(90^{\circ}, 0\right), \\
s_{2}=I\left(45^{\circ}, 0\right)-I\left(135^{\circ}, 0\right), \\
s_{3}=I\left(45^{\circ}, \pi / 2\right)+I\left(135^{\circ}, \pi / 2\right) .
\end{array}\right\}
$$




\section{APPENDIX II-QUARTER WAVEPLATE}

Consider a wave incident on a waveplate made of a uniaxial crystal that is oriented with its optic axis perpendicular to the direction of propagation of the wave. The field component parallel to the optic axis is an extraordinary wave, and the field component perpendicular to the optic axis is an ordinary wave. Let $n_{e}$ and $n_{o}$ be the indices of refraction for the extraordinary and ordinary waves respectively. If $n_{e}>n_{0}$, the crystal is positive uniaxial, and the optic axis has a slower phase velocity than the perpendicular axis. If $n_{e}<n_{o}$, the crystal is negative uniaxial, and the optic axis has a faster phase velocity than the perpendicular axis. The waveplates used in this experiment are zero-order quartz (positive uniaxial crystal) waveplates.

Denote the axes with the slower and faster phase velocities with the unit vectors $e_{s}$ and $e_{f}$, respectively. For a field $\hat{F}_{1}$ incident on the crystal, the transmitted field, $\hat{\boldsymbol{F}}_{2}$, is

$$
\hat{F}_{2}=e_{s}\left(e_{s} \cdot \hat{F}_{1}\right) e^{i \varphi_{s}}+e_{f}\left(e_{f} \cdot \hat{F}_{1}\right) e^{i \phi_{s}} .
$$

Here the caret indicates a complex field of the form $\hat{F}=F e^{i\left(\omega+\delta_{1}\right)} . \phi_{s}$ and $\phi_{f}$ are the phase shifts along the slow and fast axes. $\delta_{1}$ is the initial phase of the wave. For a plate of thickness $d$

$$
\left.\begin{array}{l}
\phi_{s}=n_{s} \omega d / c, \text { and } \\
\phi_{f}=n_{f} \omega d / c .
\end{array}\right\}
$$

$n_{s}$ is the index of refraction along the slow axis, and $n_{f}$ is the index of refraction along the fast axis. $c$ is the speed of light in a vacuum. 
Introducing the phase factor $\delta$, we can relate the entire phase delay to the slow axis.

$$
\hat{\boldsymbol{F}}_{2}=e_{s}\left(e_{s} \cdot \hat{\boldsymbol{F}}_{1}\right) \mathrm{e}^{i(\phi+\delta)}+e_{f}\left(\boldsymbol{e}_{f} \cdot \hat{\boldsymbol{F}}_{1}\right) \mathrm{e}^{\mathrm{i} \delta}
$$

Here $\phi$ is the phase retardation

$$
\phi=\phi_{s}-\phi_{f}
$$

Now let the incident wave be linearly polarized along the $x$-axis, and let the slow axis make an angle $\theta$ with the $x$-axis. Thus $\hat{F}_{1}=\hat{F}_{1} e_{x}$, and

$$
\left.\begin{array}{l}
e_{s}=\cos \theta e_{x}+\sin \theta e_{y}, \text { and } \\
e_{f}=-\sin \theta e_{x}+\cos \theta e_{y} .
\end{array}\right\}
$$

The output field becomes

$$
\hat{F}_{2}=\hat{F}_{1}\left[\left(\left(\cos ^{2} \theta \cos \phi+\sin ^{2} \theta\right)+i \cos ^{2} \theta \sin \phi\right) e_{x}+\frac{1}{2} \sin 2 \theta\left(-2 \sin ^{2} \frac{\phi}{2}+i \sin \phi\right) e_{y}\right] e^{i \delta} .
$$

For a zero-order quarter waveplate, $\phi=\frac{\pi}{2}$, and

$$
\left.\begin{array}{rl}
\hat{F}_{2} & =\hat{F}_{1}\left[\left(\sin ^{2} \theta+i \cos ^{2} \theta\right) e_{x}+\frac{1}{2} \sin 2 \theta(-1+i) e_{y}\right] e^{i \delta}, \\
& =F_{1} e^{i \omega t} e^{i\left(\delta+\delta_{1}\right)}\left(\sin ^{2} \theta+i \cos ^{2} \theta\right) e_{x}+F_{1} e^{i \omega t} e^{i \delta} \sin 2 \theta(-1+i) e_{y} .
\end{array}\right\}
$$

Now in magnitude and phase notation

$$
\sin ^{2} \theta+i \cos ^{2} \theta=\rho e^{i n}
$$

where 


$$
\left.\begin{array}{l}
\eta=\tan ^{-1}\left(\frac{\cos ^{2} \theta}{\sin ^{2} \theta}\right), \text { and } \\
\rho=\left(\sin ^{4} \theta+\cos ^{4} \theta\right)^{1 / 2} .
\end{array}\right\}
$$

Similarly $-1+i=-\sqrt{2} \mathrm{e}^{-\mathrm{i} \pi / 4}$. Thus the output field becomes

$$
\hat{F}_{2}=F_{1} \rho e^{i\left(\omega t+\eta+\delta+\delta_{1}\right)} e_{x}-\frac{F_{1}}{\sqrt{2}} \sin 2 \theta e^{i\left(\omega t-\frac{\pi}{4}+\delta\right)} e_{y} .
$$

The real field is then

$$
\left.\begin{array}{rl}
F_{2} & =\operatorname{Re}\left(\hat{F}_{2}\right), \\
& \left.=F_{1} \rho \cos \left(\omega t+\eta+\delta+\delta_{1}\right) e_{x}-F_{1} \frac{1}{\sqrt{2}} \sin 2 \theta \sin \left(\omega t-\frac{3 \pi}{4}+\delta+\delta_{1}\right) e_{y} \cdot\right\}
\end{array}\right\}
$$

The phase factor $\delta+\delta$, disappears in measurements of the irradiance and may be chosen equal to

$$
\delta+\delta_{1}=\frac{3 \pi}{4}-\frac{\eta}{2} .
$$

Thus

$$
\boldsymbol{F}_{2}=F_{1} \rho \cos (\omega t) e_{x}+F_{1} \frac{\sin 2 \theta}{\sqrt{2}} \sin (\omega t) e_{y} .
$$

Casting the field in the form of Equation (2.3.9)

$$
F_{2}=F_{2}\left[\cos (\omega t) e_{x}+\varepsilon_{2} \sin (\omega t) e_{y}\right]
$$

Here

$$
F_{2}=F_{1}\left(\sin ^{4} \theta+\cos ^{4} \theta\right)^{1 / 2}
$$

and the ellipticity of the output wave is 


$$
\varepsilon_{2}=\frac{1}{\sqrt{2}} \frac{\sin 2 \theta}{\left(\sin ^{4} \theta+\cos ^{4} \theta\right)}
$$

Figure A.II.1 shows the output ellipticity given by Equation (A.II.16).

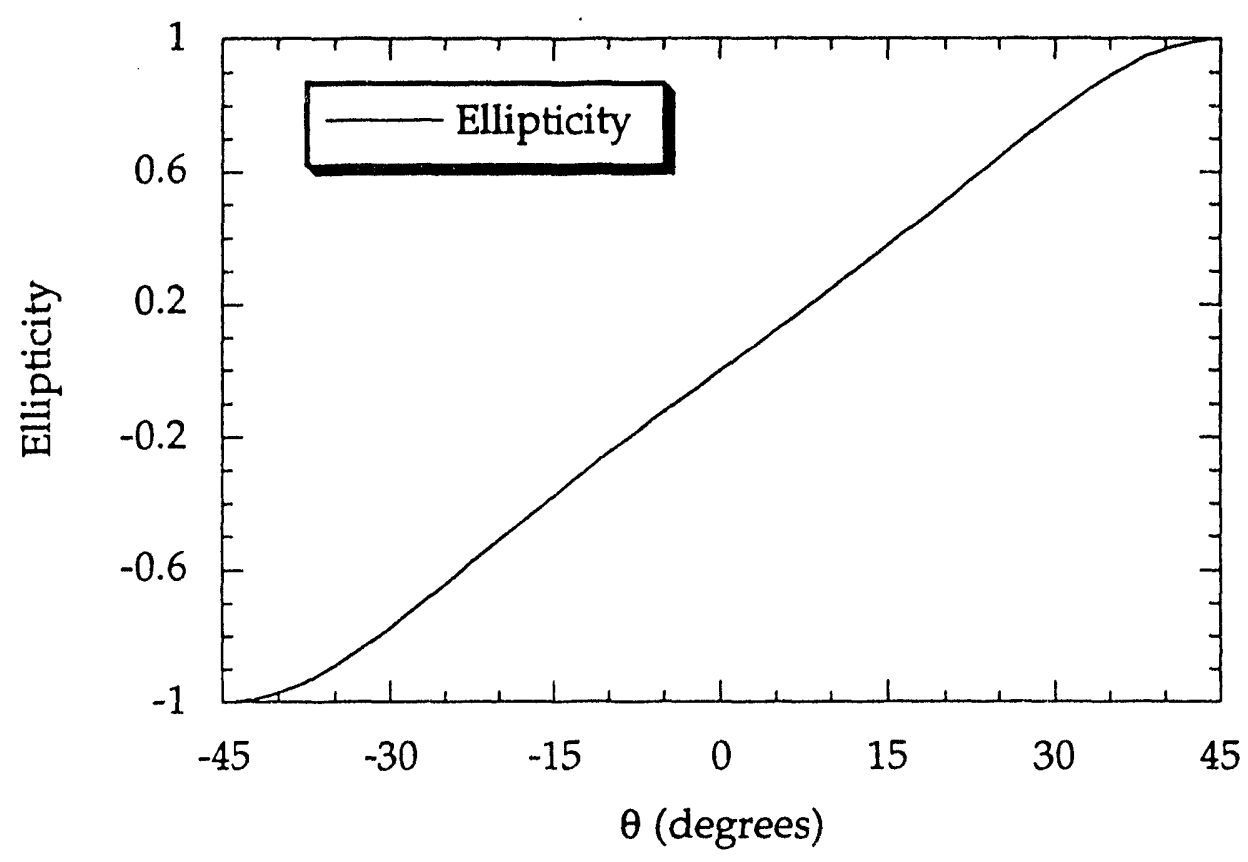

Figure A.II.1 The ellipticity of the output wave for a linearly polarized wave incident perpendicular to the optic axis of a uniaxial-crystal quarter-wave retarder as a function of $\theta$, the angle that the waveplate slow axis makes with the polarization direction of the incident wave. 


\section{APPENDIX III-THE PONDERMOTIVE POTENTIAL}

When a linearly polarized laser ionizes an atom, the ejected electron experiences an effective potential due to the electromagnetic field of the laser[81, 82]. For electrons bound to atoms, the motion of the electrons is modified by the applied field. For Rydberg states and optical fields, the pondermotive motion is essentially the classical limit of the quantum mechanical motion. Since quantum mechanics and classical mechanics yield the same value for the energy of the oscillations, I will only outline the classical derivation[83, 84]. The classical equation of motion of the electron is

$$
m \frac{d v}{d t}=-e(F+v \times B)
$$

Here $\mathrm{v}$ is the velocity of the electron, $\mathrm{F}$ is the applied electric field of the laser, and $B$ is the applied magnetic field. We let the zero-order field be represented by $F=F_{0}(r) \cos (\omega t)$, and expand the velocity and position, $\mathrm{r}$, of the electron by order

$$
\begin{aligned}
& v=v_{1}+v_{2}+\cdots \\
& r=r_{0}+r_{1}+\cdots
\end{aligned}
$$

The zero-order velocity is zero because fixed external fields are absent. Expanding $\mathrm{F}_{0}$ around $\mathrm{r}=\mathrm{r}_{0}$,

$$
F_{0}(r)=F_{0}\left(r_{0}\right)+\left(r_{1} \cdot \nabla\right) F_{0}\left(r_{0}\right)+\cdots
$$

From the Maxwell Equation $\nabla \times F=-\partial B / \partial t$, we obtain the first order magnetic field,

$$
B_{1}=-\frac{1}{\omega} \nabla \times F_{0} \sin (\omega t)
$$


Now we may set up the second order equation of motion of the electron and average over a cycle of the wave to obtain the average oscillatory force acting on the electron over a cycle.

$$
F_{p}=-\frac{1}{4} \frac{e^{2}}{m \omega^{2}} \nabla\left(F_{0}{ }^{2}\right)
$$

Defining the pondermotive potential as the potential which causes the pondermotive force through $F_{p}=-\nabla U_{p}$, we find

$$
U_{p}=\frac{1}{4} \frac{e^{2}}{m \omega^{2}} F_{0}^{2} .
$$

Numerically, we may also use the relationship between the electric field and the irradiance to show that the pondermotive potential is

$$
U_{p}(\mathrm{eV})=9.33 \times 10^{-14} I\left(\mathrm{~W} / \mathrm{cm}^{2}\right) \lambda^{2}(\mu \mathrm{m}) \text {. }
$$

The pondermotive potential relates to several important effects in high field processes $[5,6,85-89]$. First, during the laser pulse an electron freed from the ion core will oscillate with the laser field. The maximum average energy of the electron will be on the order of the pondermotive potential. Thus the electron can have an energy on the order of the pondermotive energy available for interactions with the ion core. This fact directly applies to the rescattering model for direct ionization. Second, all energy levels in an atom shift in optical fields because of AC Stark shift or induced polarizability. For sufficiently high Rydberg states optical fields produce essentially identical motion of the electron as produced for free electrons. Thus the total energy of a Rydberg state increases by nearly the pondermotive potential. This strongly influences the above-thresholdionization electron energies $[51,90]$. This effect also appears for general atomic levels as an increase in the ionization potential in Equation (2.3.4). Third, if an 
electron leaves the focus of the laser while $U_{p}$ is changing, inelastic scattering of the electrons by the photons may convert the oscillatory energy to directed kinetic energy. Since the pondermotive potential in intense laser fields can be larger than the original kinetic energy of the ejected electron, the photoelectron energy detected outside the laser beam will be much larger than the original kinetic energy of the ejected electron. Finally, since the electron velocity increases along the gradient of the focused laser irradiance, the final trajectory of the electron may be different from its original trajectory at the time of ejection.

While I have not measured electron energies, it is worth noting that very short laser pulses such as those used in these experiments minimize pondermotive effects. If the electron does not move far enough to experience the gradient in the irradiance, the pondermotive energy returns to the laser beam by stimulated scattering. Thus for short laser pulses the detected electron energy and trajectory are the actual electron energy and trajectory at the time of ionization $[90,91]$. 


\section{APPENDIX IV-THE ATOMIC UNIT OF IRRADIANCE}

The atomic unit of irradiance is frequently-and mistakenly-taken to be the irradiance of one atomic unit of linearly polarized field. That is for a field of $5.292 \times 10^{-11} \mathrm{~V} / \mathrm{m}$ the unit of irradiance is taken to be

$$
\begin{aligned}
\mathrm{I} & =\frac{1}{2 \mu_{0} c}\left(5.142 \times 10^{11} \mathrm{~V} / \mathrm{m}\right)^{2} \\
& =\frac{1}{2(376.7)}\left(5.142 \times 10^{11} \mathrm{~V} / \mathrm{m}\right)^{2} \\
& =3.51 \times 10^{20} \mathrm{~W} / \mathrm{m}^{2} \\
& =3.51 \times 10^{16} \mathrm{~W} / \mathrm{cm}^{2}
\end{aligned}
$$

This is the irradiance of a wave that has one atomic unit of electric field, but it is not the atomic unit of irradiance.

The correct conversion from atomic units may be made to CGS or MKS units. For now consider CGS units. The atomic units are based on the units of charge, mass and the unit of action or angular momentum [92]. That is

$$
\begin{aligned}
& 1 \text { au of action }=\hbar=1.0544 \times 10^{-27} \mathrm{erg} \mathrm{s} \\
& 1 \text { au of mass }=m=9.109 \times 10^{-28} \mathrm{gm} \\
& 1 \text { au of charge }=e=4.8029 \times 10^{-10} \text { e.s.u }
\end{aligned}
$$

The atomic units of length and velocity are then derivable as

$$
\begin{aligned}
& 1 \text { au of length }=a_{0}=\frac{\hbar^{2}}{m e^{2}}=5.2917 \times 10^{-9} \mathrm{~cm} \\
& 1 \text { au of velocity }=v_{0}=\frac{e^{2}}{\hbar}=2.1877 \times 10^{8} \mathrm{~cm} / \mathrm{s}
\end{aligned}
$$

The fine structure constant is 


$$
\alpha=\frac{e^{2}}{\hbar c}=\frac{1}{137.037}
$$

From these definitions, the atomic units of energy and time are given by

$$
\begin{aligned}
& 1 \text { au of energy }=\frac{e^{2}}{a_{0}}=4.359 \times 10^{-11} \mathrm{erg}, \\
& 1 \text { au of time }=\frac{a_{0}}{v_{0}}=2.4189 \times 10^{-17} \mathrm{~s} .
\end{aligned}
$$

The atomic unit of irradiance is then

$$
\begin{aligned}
1 \text { au of irradiance } & =\frac{\text { lau energy }}{(1 \text { au length })^{2}(1 \text { au time })} \\
& =\frac{e^{2} / a_{0}}{a_{0}^{2} a_{0} / v_{0}} \\
& =6.436 \times 10^{22} \frac{\mathrm{erg}}{\mathrm{cm}^{2} \mathrm{~s}}
\end{aligned}
$$

in CGS units. Since $1 \mathrm{erg}=10^{-7} \mathrm{~J}$, the value for an atomic unit of irradiance in $\mathrm{W} / \mathrm{cm}^{2}$ is

$$
1 \text { au of irradiance }=6.436 \times 10^{15} \frac{\mathrm{W}}{\mathrm{cm}^{2}} \text {. }
$$

Thus the irradiance of a linearly polarized wave with one atomic unit of field-as given by Equation (A.IV.1)-is 5.45 au and not 1 au. This is consistent with the form of the irradiance for atomic units given in Equation (2.7.7) which is

$$
I=\frac{1}{8 \pi \alpha}\left(1+\varepsilon^{2}\right) F_{0}^{2} \quad \text { (atomic units) }
$$

For a linearly polarized wave with one atomic unit of electric field $\left(F_{0}=1, \varepsilon=0\right)$, the irradiance is

$$
I=\frac{1}{8 \pi \alpha} \mathrm{au}=\frac{137.037}{8 \pi} \mathrm{au}=5.45 \mathrm{au} .
$$


The form for the irradiance in atomic units given in Equation (A.IV.8) may also be derived from the expression in CGS units

$$
I=\frac{c}{8 \pi}\left(1+\varepsilon^{2}\right) F_{0}^{2}
$$

by noting that in atomic units

$$
\alpha=\frac{e^{2}}{\hbar c}=\frac{1}{c}
$$

and directly substituting for $c$. 

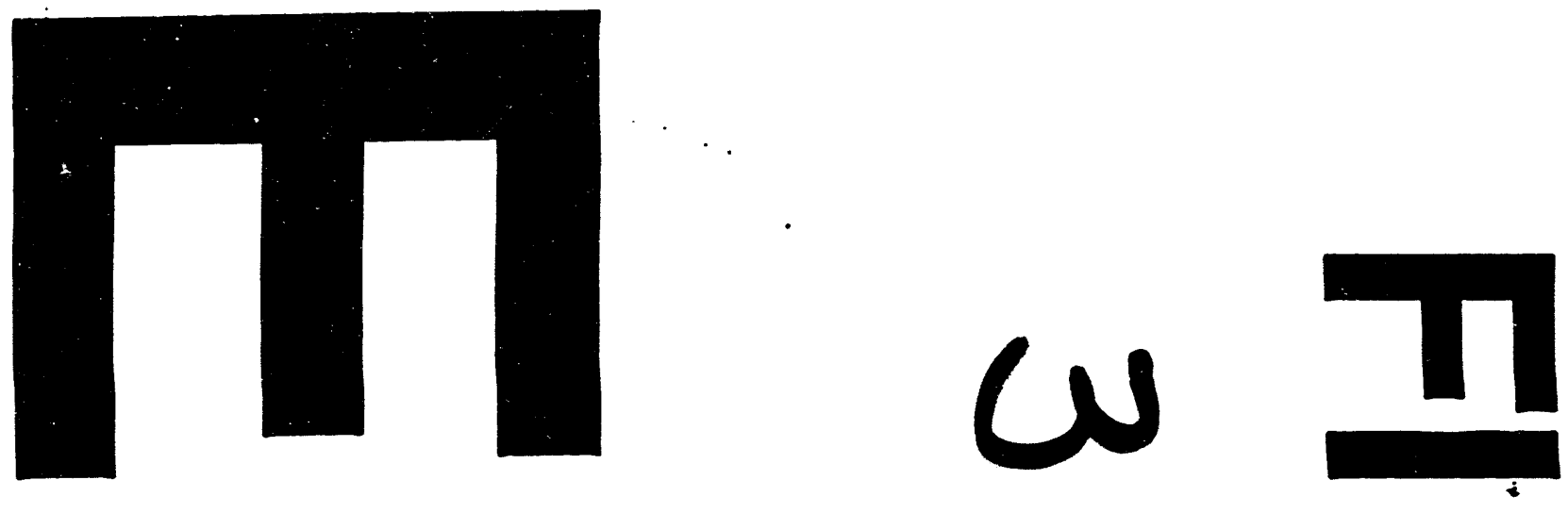

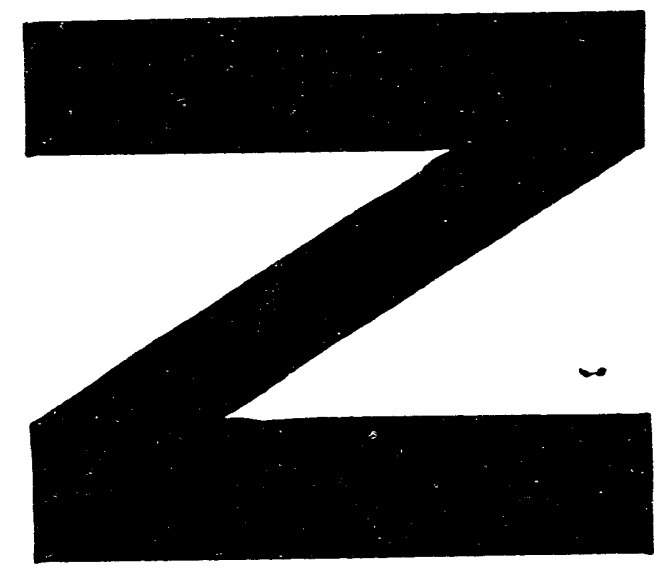

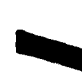
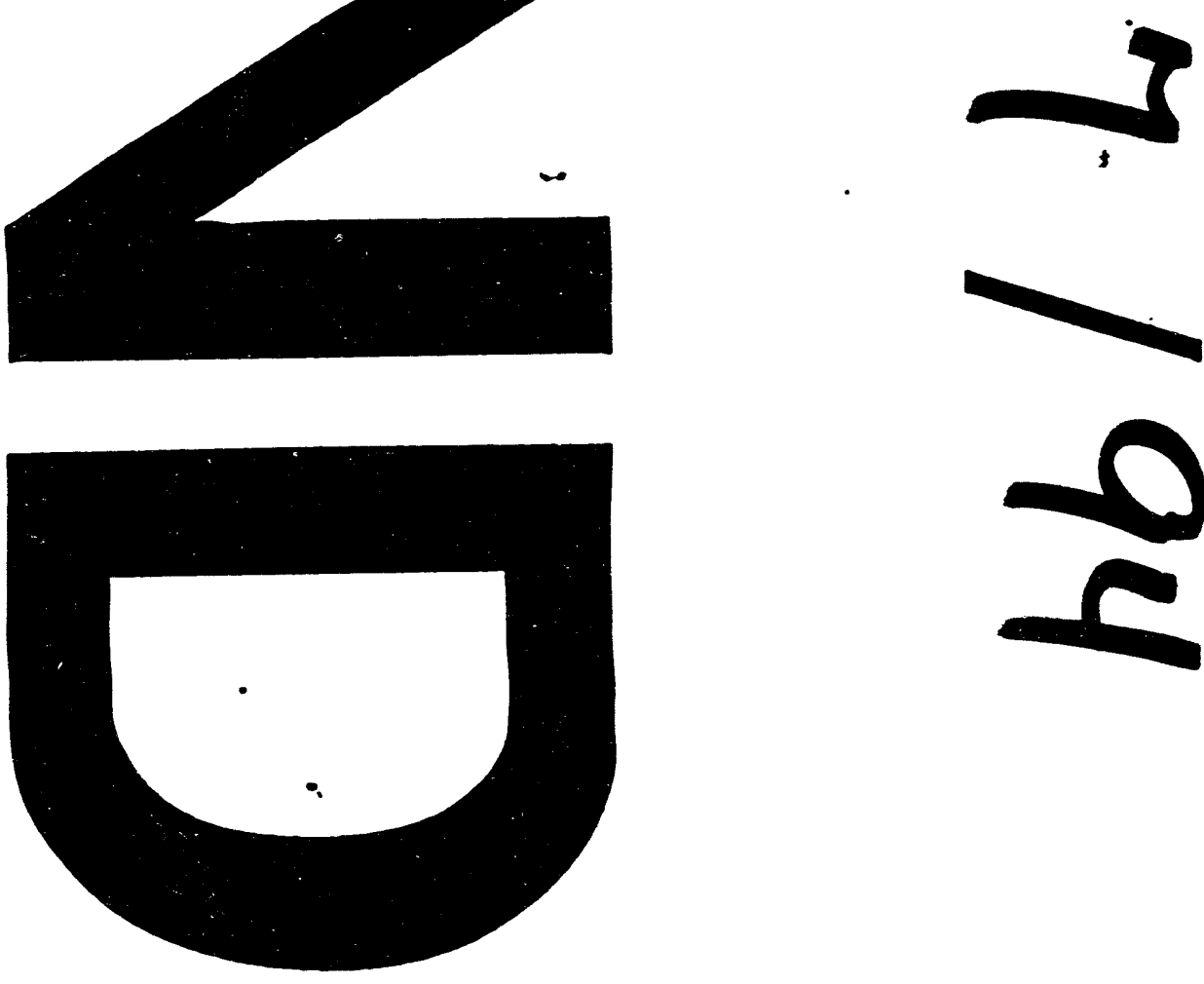

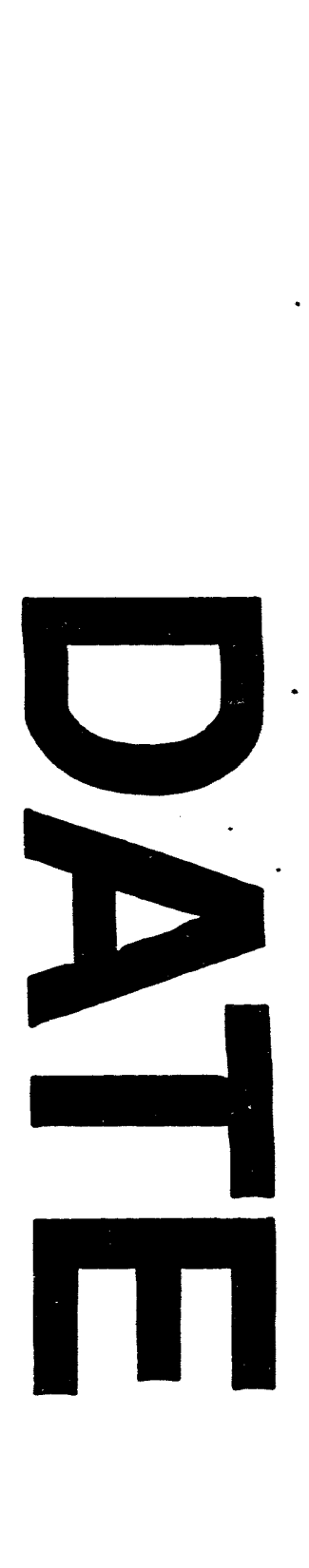




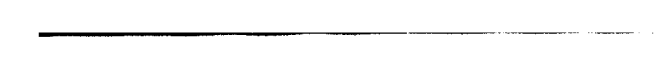 \\ -}

